\title{
10. COMPARISON OF MAJOR-AND TRACE-ELEMENT GEOCHEMISTRY OF ABYSSAL PERIDOTITES AND MAFIC PLUTONIC ROCKS WITH BASALTS FROM THE MARK REGION OF THE MID-ATLANTIC RIDGE ${ }^{1}$
}

\author{
John F. Casey ${ }^{2}$
}

\begin{abstract}
Holes 920B, 920D, 921A, 921B, 921C, 921D, 921E, 922A, 922B, and 923A were drilled into crust of the Mid-Atlantic Ridge near the Kane Fracture Zone (MARK) area during Leg 153 of the Ocean Drilling Program. Holes 920B and 920D were drilled into an ultramafic massif and Sites 921,922, and 923 were drilled into a gabbroic massif, both of which are located on the western rift valley wall of the Mid-Atlantic Ridge south of the Kane Transform. Bulk-rock major-, trace-, and rare-earth element (REE) analyses of diabasic, gabbroic, and ultramafic rocks recovered from these holes are reported here. These bulk analyses are augmented by the results of mineral chemistry studies on a subset of the same samples.

Large ranges in bulk-rock and mineral chemistry are documented from all rock types. Ultramafic rocks in Holes $920 \mathrm{~B}$ and 920D are interpreted to be dominantly residual mantle, but they include variably fractionated ultramafic and mafic cumulates that have intrusive contacts with the residual mantle harzburgites. Bulk-rock major- and compatible trace-element abundances, as well as petrographic data for residual harzburgites, indicate that a fertile MORB mantle was depleted by $\sim 15 \%$ to $20 \%$ partial melting or $10 \%-15 \%$ if a more depleted mantle source, such as Tinaquillo Lherzolite, is chosen. The mean extent of melting is likely to have been approximately half of the maximum value computed based on the residuum. Incompatible traceelement data show, however, that this residuum may have been part of an open system and refertilized at late stages by melts flowing through a locally porous matrix and later by more channelized melts (veins) as the residuum became part of the mechanical lithosphere. The crystallization products of these late melts include disseminated magmatic clinopyroxene and narrow veins or composite veins of dunite, wehrlite, pyroxenite, and gabbroic rocks. Ultramafic vein samples are variably depleted to enriched in incompatible elements and span a wide range of fractionation extents based on bulk-rock and mineral chemistry. Melts calculated to have been in equilibrium with clinopyroxene in ultramafic and mafic samples from Site 920 vary widely. They are dominantly ultradepleted, but include some samples that are enriched in incompatible elements (Na and $\mathrm{Ti}$ ) with respect to MARK basalts, glasses, and Leg 153 diabases. The range in composition cannot simply be explained by crystal fractionation of a single parental magma, but requires a broad range of parental melts or their derivatives to be in equilibrium with clinopyroxene. Bulk-rock and mineral chemistry studies of residual and cumulate ultramafic rocks support the notion of an open-system, near-fractional mantle melting column. The residual peridotites were also cut by late-stage, variably altered, high$\mathrm{MgO}(13-15 \mathrm{wt} \%$ ) diabase dikes with quenched margins.

Gabbroic samples from Sites 921,922 , and 923 drilled within the gabbroic massif likewise cover a broad spectrum of lithologies and compositions, and include troctolites, olivine gabbros, gabbros, oxide gabbros, felsic diorites, and quartz diorites. Melt compositions calculated to be in equilibrium with gabbroic clinopyroxene include melts that range from those that are significantly more fractionated to less fractionated than basaltic glasses from the MARK area, but also show a smaller range of parental melts in gabbroic samples when compared to the range documented in Site 920 ultramafic and mafic samples. Hole $923 \mathrm{~A}$, in which recovery was high, shows clear evidence of downhole cryptic chemical variation consistent with recharge and magma mixing within subaxial magma chambers. In addition, bulk-rock REE abundances in gabbroic samples show both enriched and depleted light REE (LREE) patterns. The LREE abundances range from less than $1 \times$ chondrite to $>100 \times$ chondrite in gabbroic samples. MARK basaltic rocks cover a much narrower range, from 6 to $24 \times$ chondrite. The chondritenormalized $\mathrm{La} / \mathrm{Yb}$ ratios of plutonic rocks vary from 0.3 to 2.3 , whereas MARK basalts have a narrower range, from 0.5 to 1.2 . Extended REE patterns for plutonic rocks tend to be spiked with $\mathrm{Sr}$ and Eu anomalies for all samples, and both positive and negative $\mathrm{Zr}$ and $\mathrm{Ti}$ anomalies for primitive and evolved samples, respectively. The range of incompatible trace-element depletions and enrichments in plutonic samples, in part, reflects modal variations in clinopyroxene and plagioclase, the abundance of trapped intercumulus melt, and a range of parental melt compositions input into the subaxial plumbing system. Ultramafic and plutonic rock compositions are interpreted to indicate that MORB erupted at the surface in the MARK region represents the more homogeneous hybrid derived by mixing of a diverse set of liquids generated within the mantle melting column or that evolved in plutonic environments in the crust or mantle.
\end{abstract}

\section{INTRODUCTION}

The Mid-Atlantic Ridge at the Kane Transform (MARK area) is one of the most extensively studied regions of the Mid-Atlantic Ridge. Several field studies, including submersible and dredging programs, have yielded extensive collections of basaltic, gabbroic, and

'Karson, J.A., Cannat, M., Miller, D.J., and Elthon, D. (Eds.), 1997. Proc. ODP, Sci. Results, 153: College Station, TX (Ocean Drilling Program).

${ }^{2}$ Department of Geosciences, University of Houston, Houston, TX 77204, U.S.A. jfcasey@uh.edu ultramafic rocks during numerous dredge and submersible cruises to the region (e.g., Melson et al., 1968; Miyashiro et al., 1969; Bryan et al., 1981, 1994; Karson and Dick, 1983; Karson et al., 1987; Gente et al., 1989; Mével et al., 1991; Cannat et al., 1993). Karson and Dick (1983) reported the discovery of a large gabbroic massif in the MARK area along the western rift valley wall near the inside corner of the ridge-transform intersection with the Kane Transform. Later, during an Alvin submersible program, Karson et al. (1987) and Casey (1986) reported the discovery of an adjacent ultramafic massif along the western rift valley wall to the south of the gabbroic massif. At the time of this discovery, ongoing drilling during Leg 109 to the MARK 
region was diverted from basaltic objectives to take advantage of these discoveries and drill ultramafic outcrops (Hole 670A). This hole demonstrated the drillability of the ultramafic massif (Detrick et al., 1988), as rapid penetration was achieved in only a few days of drilling. During numerous submersible and camera expeditions to the region, these massifs have been extensively mapped and sampled (e.g., Karson and Dick, 1983; Karson et al., 1987; Gente et al., 1989, 1995; Cannat et al., 1993).

These studies provided the opportunity to develop a program of offset drilling to obtain in situ samples from the gabbroic and ultramafic massifs along a rift valley that were distal from a major transform valley. Most previous collections of oceanic plutonic and residual peridotite samples have been recovered within transform valleys (Dick, 1989). The advantages of obtaining continuous to semicontinuous core of ultramafic and gabbroic samples in the MARK area are that (1) the details of the geologic and spatial relationships are often preserved among samples recovered, (2) direct comparisons are facilitated between abundant ultramafic, gabbroic, and basaltic rocks at essentially a single location along the Mid-Atlantic Ridge, and (3) comparisons of these ridge axes collections with the more dominant oceanic dredge collections (e.g., see review of Dick, 1989) of ultramafic and gabbroic lithologies from transform valleys are possible. During Ocean Drilling Program (ODP) Leg 153, drilling was highly successful in recovering abundant core from both the ultramafic massif at Site 920 (Holes 920B and 920D) and from the gabbroic massif at Sites $921,922,923$, and 924 along the western rift valley wall in the MARK area. Site 920 is located proximal to the first second-order segment discontinuity (at $\sim 23^{\circ} 10^{\prime} \mathrm{N}$ ) south of the Kane Transform, and Sites $921,922,923$, and 924 are located in close proximity to each other near the center of the first second-order segment south of the Kane Transform (Figs. 1A, 1B). A full description of the tectonic setting of each hole, its position, and core recovered is contained within Cannat, Karson, Miller, et al. (1995). Core recovery and unit definitions for each hole included in this study are summarized in Figures $2 \mathrm{~A}$ and $2 \mathrm{~B}$.

In this paper, results of bulk-rock major-, trace-, and rare-earth element (REE) analyses, as well as mineral chemistry analyses of Leg 153 gabbroic and ultramafic rocks, are presented for various holes at Sites $920,921,922$, and 923 . These results are compared with bulkrock and glass geochemical studies of basalts recovered during previous cruises (Fig. 1B) to the MARK region (e.g., Bryan et al., 1981, 1994; J.F. Casey, W. Bryan, and G. Thompson, unpubl. data). These comparisons are made in an effort to more fully evaluate petrogenetic processes that occur in the subaxial mantle and plutonic environments and that ultimately lead to the formation of N-type mid-ocean ridge basalt (MORB) erupted at the surface in the MARK region.

This comparison between mantle-plutonic and basaltic components of the lithosphere at a single locality facilitates a more comprehensive evaluation of (1) the nature of the melting regime, (2) the extent of melting, (3) melt segregation processes, (4) the extent and location of magma mixing and pooling of mantle melts, (5) the extent, nature, and regime of magma evolution in subaxial magma chambers, and (6) crustal formation and architecture along a slow-spreading center characterized by low melt supplies. In addition, with the exception of results from ODP Hole 735B (Dick et al., 1991), very few comprehensive studies of the major-element, trace-element, and REE bulk-rock chemistry of large suites of samples from the plutonic foundations of the oceanic crust have been completed, yet they are generally believed to form two-thirds of the oceanic crust. This study largely constitutes an effort to more fully characterize the major- and trace-element compositions in the plutonic component of the lithosphere. Its composition has significance not only to petrogenetic processes related to the formation of oceanic crust and genesis and evolution of MORB, but also to the nature of subducted oceanic lithosphere, oceanic slab-derived components associated with magmatism in arc settings, and lithosphere recycling.

\section{ANALYTICAL METHODS}

A total of 250 samples were analyzed during this study. Of these, 150 samples of gabbroic, ultramafic, and diabasic samples from Sites $920,921,922$, and 923 were analyzed for bulk-rock major- and traceelement compositions during the study. REE analyses were conducted for all mafic gabbroic and diabasic samples. The majority of residual ultramafic rocks have REE abundances below instrumental detection or quantitation limits for the analytical techniques used (see below), and REE analyses are reported only for nonresidual pyroxenite, wehrlite, gabbroic and diabasic intrusive rocks within residual harzburgites, because only these samples tended to have higher and measurable abundances. In addition to the 150 samples analyzed for major and trace elements, splits of all Leg 153 gabbroic, and diabasic powders analyzed by shipboard X-ray fluorescence (XRF) on the JOIDES Resolution were analyzed for trace elements and REEs.

Bulk-rock analyses were conducted on a Thermo-Jarrell Ash Sequential inductively coupled plasma-atomic emission spectroscopy (ICP-AES) at the University of Houston using methods described in Lytwyn and Casey (1993, 1995), Smith (1994), and C. Xia and J.F. Casey, (unpubl. data). Precision and accuracy of measurements are reported in Smith (1994). Major- and trace-element analyses were conducted on dilute solutions after fusion with lithium metaborate. REE, Sc, and Y analyses were conducted after fusion and preconcentration via liquid chromatography. For mafic samples, oxide wt $\%$ of $\mathrm{Si}, \mathrm{Ti}, \mathrm{Al}, \mathrm{Fe}, \mathrm{Mn}, \mathrm{Mg}, \mathrm{Ca}, \mathrm{Na}, \mathrm{K}, \mathrm{P}$ and elemental abundances in ppm of V, Ti, Cr, Sr, Zr, Ba, Sc, Y, La, Ce, Nd, Sm, Eu, Gd, Dy, Er, $\mathrm{Yb}, \mathrm{Lu}$ are reported. $\mathrm{Co}, \mathrm{Cu}, \mathrm{Zn}$ are also reported for a subset of the samples. For most ultramafic samples, all elements above except for the REE, Sc, and $\mathrm{Y}$ are reported.

Every effort was made to avoid or eliminate hydrothermal veins in sampling for bulk-rock analyses. Very thin magmatic veins (e.g., clinopyroxenites and websterites) within residual harzburgites that were included for bulk-rock analyses inevitably involved sampling some of the olivine-rich wallrock with the pyroxenite to various extents because of the small nature of the samples analyzed. The boundaries between the olivine-rich wallrock and the veins were also commonly gradational, further complicating the separation between the wallrock and the vein.

Loss on ignition (LOI) is reported for each sample. Because of the high volatile contents of ultramafic rocks (up to $16 \mathrm{wt} \% \mathrm{H}_{2} \mathrm{O}$ ), all trace-element analyses were conducted on fired sample powders. A similar procedure was followed during shipboard XRF analyses based on comparison of analyses of fired and unfired samples (Shipboard Scientific Party, 1995; fig. 27, p. 64). Analyses are, therefore, directly comparable and eliminate reductions in trace-element abundances caused by volatile dilution when samples are weighed without firing.

Mineral chemistry studies were conducted on a Cameca SX-50 four-spectrometer microprobe at Rice University. Measured concentrations were corrected for atomic number, absorption, and fluorescence affects by the ZAF method. Standards consisted of natural mineral standards from the Smithsonian collection and commercially prepared standard blocks. Beam diameters were $5 \mu \mathrm{m}$ for olivine, plagioclase, and spinel, but increased to $20 \mu \mathrm{m}$ for clinopyroxene and orthopyroxene. Broad beam analysis was used for pyroxenes to ensure that exsolution lamellae were included in the analyzed portion of the crystal. Both cores and rims were analyzed in each sample to document the full intrasample variation. Minerals in residual ultramafic rocks tended to be homogeneous, except within and adjacent to pyroxenite and wehrlite veins, whereas gabbroic samples ranged from homogeneous to those that exhibit at least some grain or thin-sectionscale compositional variation. Olivine and clinopyroxene varied little in composition within samples, whereas plagioclase showed larger intrasample variations. 
A

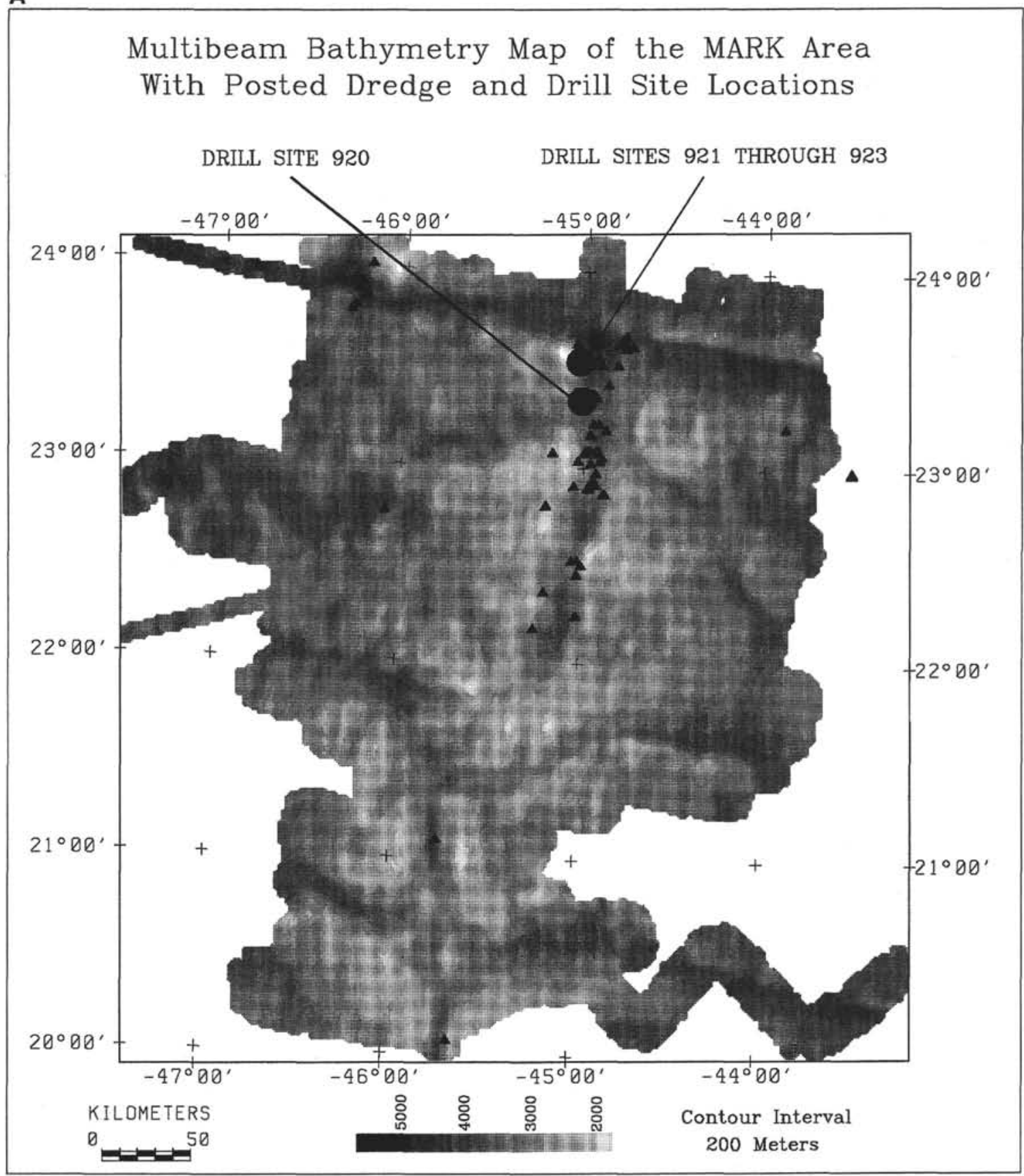

Figure 1. General bathymetric maps of (A) the Kane Fracture Zone and (B) Leg 153 sites investigated in this study. Bathymetric data from Gente et al. (1995). ODP sites are shown as labeled, solid circles. The general bathymetric map of the MARK area also includes regional sample localities of basalts from previous cruises to the region (Bryan et al., 1981; 1994) that are used for comparison with Leg 153 samples. Note that Site 920 lies near the first second-order segment boundary south of the Kane Fracture Zone and Sites 921, 922, and 923 lie with the segment center. Site 920 includes holes from the ultramafic massif, and Sites 921,922 , and 923 include holes from the gabbroic massif.

\section{THE NATURE OF THE MARK MELTING REGIME BASED ON REGIONAL GEOCHEMICAL CHARACTERISTICS OF BASALTS AND RESIDUAL PERIDOTITES}

Before presentation of the bulk of the Leg 153 data set, geochemical parameters for basaltic and peridotitic samples that are pertinent to the nature of the melting regime in the MARK area are reviewed.
To provide a framework for this study of Leg 153 plutonic and abyssal peridotite samples, we evaluate the MARK region's melting, temperature, and mantle source regime by conducting a straightforward comparison of basalts or basalt glass geochemistry and peridotite mineral chemistry from the MARK region with that from adjacent portions of the northern Mid-Atlantic Ridge. Parameters sensitive to the nature of the mantle source, the extent of melting, and the average pressure of melting are discussed below. 
B

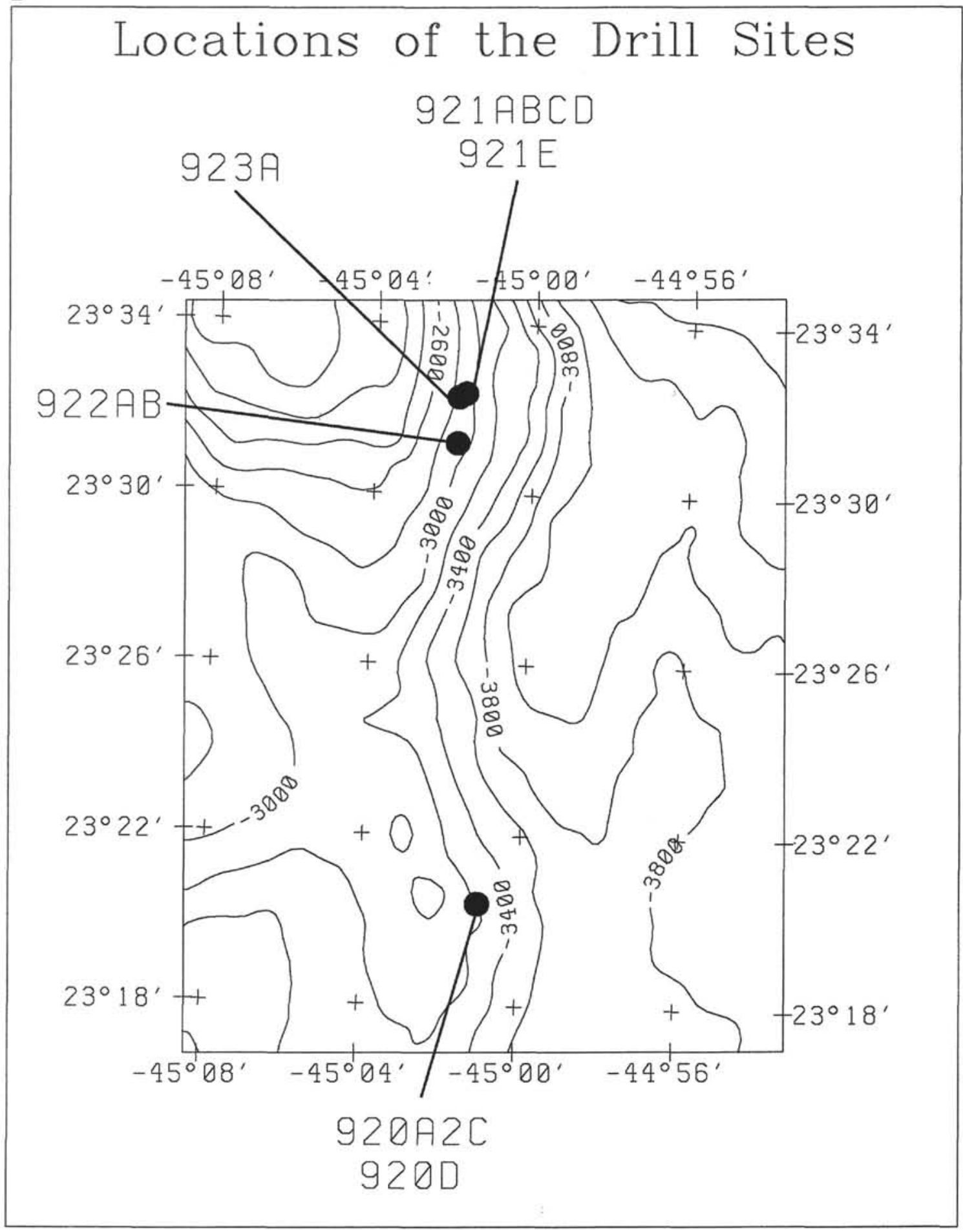

Figure 1 (continued).

\section{Basalts}

The northern Mid-Atlantic Ridge exhibits systematic long-wavelength geochemical variation in isotopic, trace-element, and majorelement geochemistry (e.g., Schilling, 1986; Schilling et al., 1983, 1995; Melson and O'Hearn, 1979, 1986; Klein and Langmuir, 1987). Bryan et al. (1994), Dosso et al. (1991, 1993), and Casey et al. (1996) have further refined this long-wavelength variation and provide added constraints to better understand the geologic and geochemical setting of the MARK region in relation to areas to the north and south.
The $\mathrm{Na}{ }_{2} \mathrm{O}, \mathrm{K} / \mathrm{Ti}, \mathrm{La} / \mathrm{Yb}$ or $\mathrm{Ce} / \mathrm{Yb}$, and ${ }^{87} \mathrm{Sr} /{ }^{86} \mathrm{Sr}$ variation in basalts vs. latitude along the Mid-Atlantic Ridge from $0^{\circ}$ to $70^{\circ} \mathrm{N}$ are shown in Figures 3A-3D. These variations show that the MARK region $\left(22^{\circ}-23^{\circ} 30^{\prime} \mathrm{N}\right)$ generally represents a region of typical $\mathrm{N}$ MORB and is characterized by relatively low $\mathrm{K} / \mathrm{Ti}, \mathrm{La} / \mathrm{Sm}$, and ${ }^{87} \mathrm{Sr} /$ ${ }^{86} \mathrm{Sr}$ (Bryan et al., 1981, 1994; Dosso et al., 1993; J.F. Casey and W.B. Bryan, unpubl. data). There is, however, a small geochemical anomaly reflected in some basaltic rocks sampled just to the south of $23^{\circ} \mathrm{N}$ near the center of a second-order segment. It is characterized by somewhat elevated $\mathrm{K} / \mathrm{Ti}(>0.2 \mathrm{wt} \%), \mathrm{La} / \mathrm{Sm}(>1.0)$, and ${ }^{87} \mathrm{Sr} /{ }^{86} \mathrm{Sr}$. 
The MARK area also represents a region where the $\mathrm{Na}_{2} \mathrm{O}$ or $\mathrm{Na}_{8.0}$ content in basalt glasses and whole rocks are high in comparison to adjacent regions. Klein and Langmuir (1987), Niu and Batiza (1991), and Langmuir et al. (1992) have indicated that high $\mathrm{Na}_{2} \mathrm{O}$ or $\mathrm{Na}_{8.0}$ in basaltic glass can be correlated with low degrees of mantle melting, based on the assumption that the initial mantle concentration of $\mathrm{Na}_{2} \mathrm{O}$ is not highly variable. The slope of the fractionation correction to $\mathrm{Na}_{8.0}$ tends to be low and the range in $\mathrm{MgO}$ content tends to be limited along the Mid-Atlantic Ridge, so we show simply the along-axis variability of raw $\mathrm{Na}_{2} \mathrm{O}$ in basaltic glass along the Mid-Atlantic Ridge (Fig. 3). $\mathrm{FeO}$ and $\mathrm{Fe}_{8.0}$ are thought to be sensitive to the average pressure of melting and/or the extent mantle depletion (Langmuir et al., 1992). The MARK region is also characterized by low $\mathrm{Fe}_{8.0}$ in basalt glass, which may be correlated with average pressure of melting (Klein and Langmuir,1987) or can be inversely correlated with the fertility of the mantle source region before melting (Langmuir et al., 1992). Low $\mathrm{Fe}_{8.0}$ is generally interpreted to indicate a low pressures of initial and average melting or prior depletion of the mantle. Our trace-element modeling of diabases and basalt from the MARK area (presented below) is consistent with melting at lower pressures within the spinel facies. We assume that the low $\mathrm{FeO}$ content is related to the pressure of melting of a typical MORB fertile mantle. Assuming $\mathrm{Na}_{2} \mathrm{O}$ and $\mathrm{FeO}$ in MARK glasses reflect the extent and average pressure of melting under spinel facies melting of a mantle source such as MORB Pyrolite-90 (MPY-90) (e.g., Falloon and Green, 1987), the modeling results of Niu and Batiza (1991) can be used to help constrain melting parameters. The MPY-90 mantle source is likely to characterize the MARK melting regime (Bryan et al., 1981; 1994). The field of MARK glasses is displayed in Figure 4 on a binary plot of $\mathrm{FeO}$ and $\mathrm{Na}_{2} \mathrm{O}$. Also plotted are available analyses of MORB glasses from the northern Mid-Atlantic Ridge. Several highly altered $\mathrm{MgO}$-rich diabases sampled during Leg 153 drilling (see below) are also plotted for comparison. The primitive (high $\mathrm{MgO}$, low $\mathrm{FeO}$ ) MARK glasses tend to have some of the higher $\mathrm{Na}_{2} \mathrm{O}$ contents in the North Atlantic, and lie somewhat below those of the Cayman Trough and Equatorial Atlantic. The modeling results of Niu and Batiza (1991) for $\mathrm{Na}_{2} \mathrm{O}$ and $\mathrm{FeO}$ content of linearly pooled near-fractional melts are also shown for comparison. The modeling is based on varying extents and pressures of melting of a fertile MORB mantle (MPY-90) source. Based on these comparisons, the MARK parental basalts can be interpreted to have originated by shallow depths of initial melting ( $\mathrm{P} i \approx 15-20 \mathrm{kbar})$ in the spinel facies as the result of $\sim 13 \%-15 \%$ average partial melting of the mantle.

Thus, compared to evidence from other basalt glasses, the MARK region appears to represent one of the four well-defined minima in the extent of melting and the average pressure of the melting along the northern Mid-Atlantic Ridge between $0^{\circ}$ and $70^{\circ} \mathrm{N}$ (Fig. 3). Based on the $\mathrm{Na}_{2} \mathrm{O}$ contents, other comparable minimum melting regions include intervals between $50^{\circ}$ and $52^{\circ} \mathrm{N}$, between $8^{\circ}$ and $11^{\circ} \mathrm{N}$, and between $1^{\circ} \mathrm{N}$ and $1^{\circ} \mathrm{S}$. The region between $1^{\circ} \mathrm{N}$ and $1^{\circ} \mathrm{S}$ along the large offset Romanche Transform appears to represent the region of least mantle melting or highest fertility in the North Atlantic (Bonatti et al., 1993; Schilling et al., 1995).

\section{Residual Peridotites}

Mineral chemistry results of abyssal peridotites from Leg 153 Holes 920B and 920D (this study), previous MARK results (Komor et al., 1990; Juteau et al., 1990), other studies (Michael and Bonatti, 1985; Bonatti and Michael, 1989; Bonatti et al., 1993; Cannat, 1993; Cannat and Casey, 1995), and our unpublished data along the northern Mid-Atlantic Ridge (J.F. Casey and S. Silantyev, unpubl. data) are included here in a compilation of data for Mid-Atlantic Ridge abyssal peridotites between $0^{\circ}$ and $45^{\circ} \mathrm{N}$ (Fig. 5). Several of these studies have shown that incompatible and compatible elements and ratios of mantle-equilibrated mineral phases in abyssal peridotites, including olivine, pyroxene, and spinel, change in a systematic way with the extent of partial melting or depletion of the mantle (Jaques and Green, 1980; Dick and Bullen, 1984; Dick et al., 1984; Michael

\section{A Leg 153 Ultramafic Massif Holes}
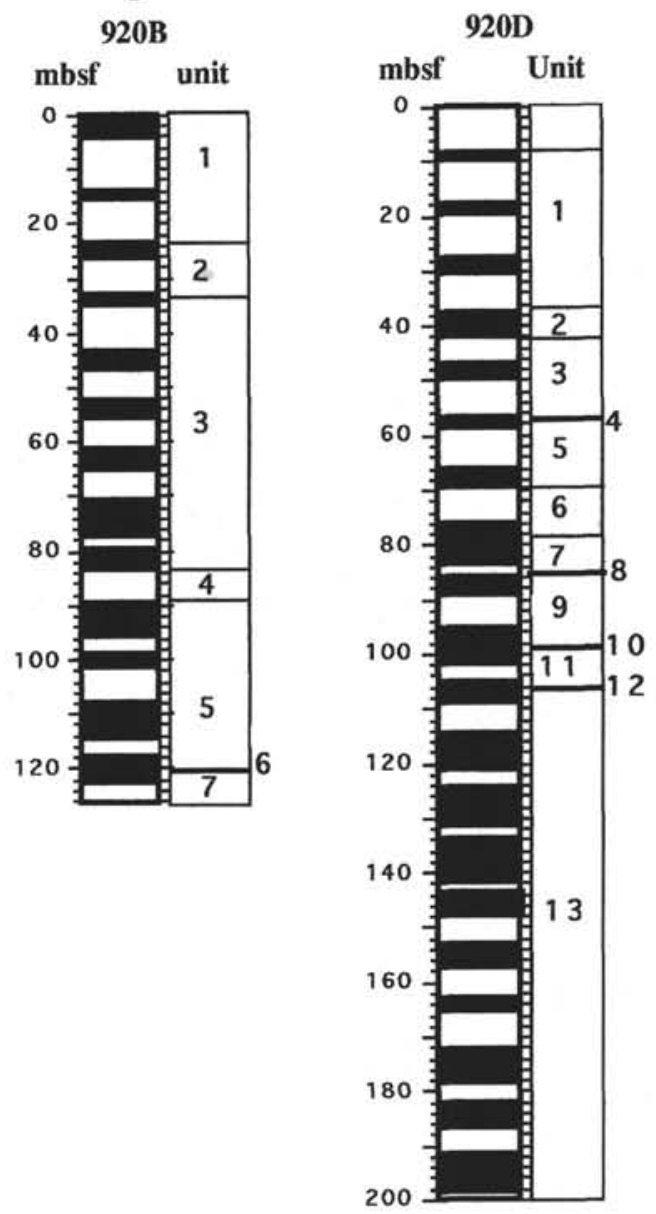

Figure 2. Core recovery intervals (black) plotted as cumulative section lengths projected from the top of each core and the distribution of lithologic unit numbers (defined below) for each hole investigated in this study. Holes 920B and 920D drilled within the ultramafic massif are shown in (A), and holes at Sites 921, 922, and 923 within the gabbroic massif are shown in (B). Lithologic units for each hole are defined as follows: Hole 920B: 1 = serpentinized harzburgite, 2 = pyroxene-rich serpentinized harzburgite, $3=$ serpentinized harzburgite, $4=$ moderately porphyritic diabase, $5=$ serpentinized harzburgite, $6=$ oxide-rich metagabbro, $7=$ amphibolite and gneissic gabbro; Hole 920D: 1 = serpentinized harzburgite, 2 = pyroxene-rich serpentinized harzburgite, 3 = serpentinized harzburgite, 4 = metagabbro and amphibolitized microgabbro, 5 = serpentinized harzburgite, $6=$ plagioclase-olivine phyric diabase, $7=$ serpentinized harzburgite (and dunite), $8=$ rodingitized gabbro, 9 = serpentinized harzburgite, 10 = pegmatitic oxide-rich gabbro, 11 = serpentinized harzburgite, 12 = oxide-rich metagabbro, $13=$ serpentinized harzburgite; Hole 921A: 1 = olivine gabbro; Hole 921B: 1 = cataclastic gabbro, 2 = aphyric to sparsely phyric diabase, 3 = lineated gabbro, $4=$ poikilitic olivine gabbro; Hole $921 \mathrm{C}$ : 1 = cataclastic gabbro, 2 = aphyric to sparsely phyric diabase, 3 = lineated gabbro, 4 = poikilitic olivine gabbro; Hole 921D: 1 = mixed gabbro/olivine gabbro; Hole 921E: 1 = very coarse grained to pegmatitic gabbro, 2 = heterogeneous poikilitic olivine gabbro, 3 = varitextured gabbro/olivine gabbro; Hole 922A: 1 = metatroctolite, 2 = troctolite and olivine gabbro; Hole 922B: 1 = troctolite and olivine gabbro, 2 = poikilitic olivine gabbro; Hole 923A: 1 = variably deformed gabbro and olivine gabbro, 2 $=$ interlayered troctolite and olivine gabbro, 3 = poikilitic olivine gabbro and troctolite, 4 = varitextured gabbro and olivine gabbro, $5=$ troctolite and poikilitic olivine gabbro. 
B

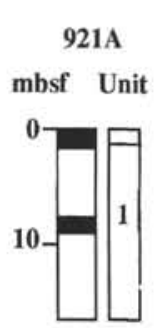

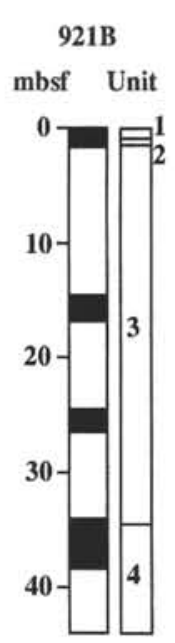

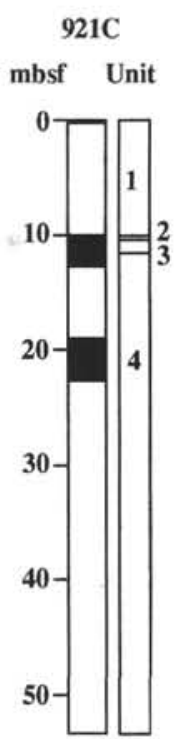

Leg 153 Gabbroic Holes
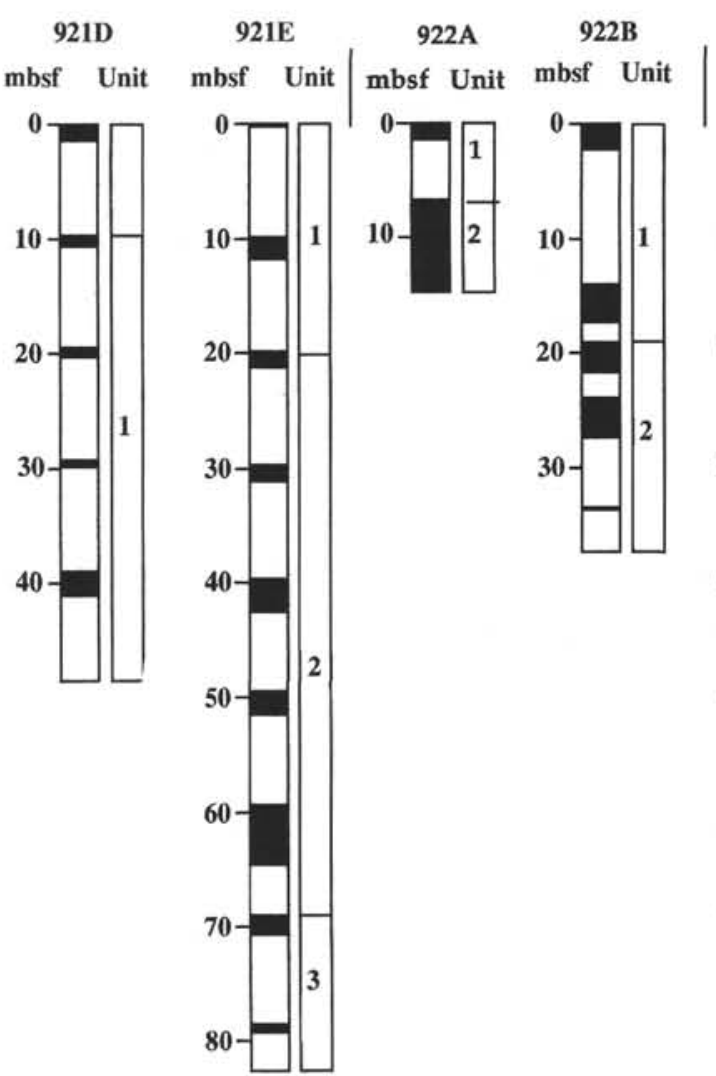

923A mbsf Unit
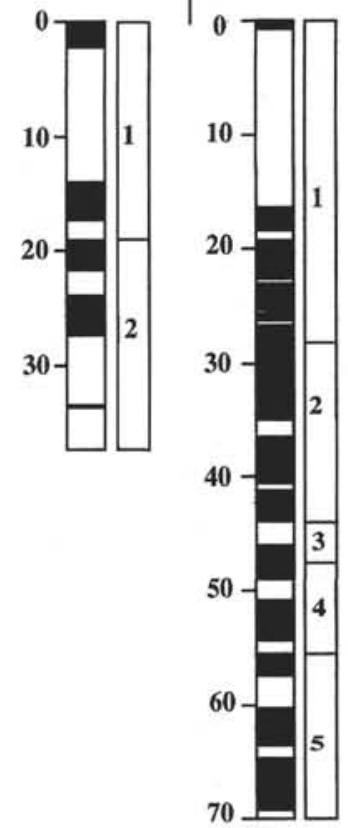

Figure 2 (continued).

and Bonatti, 1985; Dick, 1989). In particular, the $\mathrm{Al}_{2} \mathrm{O}_{3}$ content of orthopyroxene and clinopyroxene should decrease, and $\mathrm{Cr} \#(100 \times \mathrm{Cr} /$ $[\mathrm{Cr}+\mathrm{Al}])$ ratios of spinel should increase as a consequence of increased extents of melting, although subsolidus reequilibration and low-temperature alteration can influence these ratios. Careful studies, however, have shown that these parameters can be used to establish the overall extent of mantle melting and depletion in abyssal peridotites. They also correlate with modal variations expected during increased extents of melting (e.g., Dick and Fisher, 1984; Dick et al., 1984; Michael and Bonatti, 1985; Dick, 1989). Figure 5 shows that the MARK residual abyssal peridotites are characterized by low $\mathrm{Cr} \#$ ratios in spinels $(\sim 20-40)$ and high $\mathrm{Al}_{2} \mathrm{O}_{3}$ content in orthopyroxene (orthopyroxene) (3.5-5 wt \%) and clinopyroxene (4.5-6 wt \%) when compared with other Mid-Atlantic Ridge residual peridotites. The along-axis variations correlate in a straightforward way with the major-element variations of basalt glasses (Fig. 3) along the northern Mid-Atlantic Ridge. Based on a fertile MORB mantle like MPY-90, our estimates of the extent of melting based on reconstructed modes and mineral chemistry indicate approximately $15 \%-20 \%$ partial melting of the mantle in the MARK region. These results are comparable to estimates based on bulk geochemistry presented below and are also similar to results obtained by Komor et al. (1990) for Hole 670 , Leg 109 abyssal peridotites from the MARK area using similar MORB pyrolite source (18\%-19\%). Estimates based on batch equilibrium melting models or accumulated near-fractional melt composition from diabases and basalts presented below do, in fact, show a somewhat lower extent of average melting (13\%-15\%) when compared to mantle peridotites $(15 \%-20 \%)$. This is not unexpected, because abyssal peridotites reside at the top of the residual melting column. They should represent lithospheric mantle that has undergone the most extensive depletion and melting $(F \max )$ (i.e., if near-frac- tional melting is operative; e.g., see Langmuir et al., 1992; Plank and Langmuir, 1993). Because accumulated near-fractional or pooled melts (i.e., the erupted MORB) are integrated over the entire melting column, MORB should reflect lower average extents of melting $(F)$ when compared to melts in equilibrium with the peridotites sampled from the top of the residual melting column. Batch equilibrium melting models or continuous melting models involving higher melt fractions are unlikely to produce such a striking relationship (e.g., see Langmuir et al., 1992).

In summary, the $\mathrm{Na}_{2} \mathrm{O}$ content of the basalts, as well as the $\mathrm{Cr} \# \mathrm{~s}$ of spinel and the $\mathrm{Al}_{2} \mathrm{O}_{3}$ content of pyroxenes within residual peridotites, are consistent and serve to define the MARK area as one of three well-defined minima in the extent of mantle depletion and melting along the Mid-Atlantic Ridge (also see, Dick et al., 1984; Dick, 1989; Michael and Bonatti, 1985; Komor et al., 1990). Basalt trace-element and isotopic data also indicate that the MARK region is generally characterized by N-MORB (Bryan et al., 1981; 1994) and that the mantle source region is likely to be depleted fertile upper mantle similar to MPY-90 (Falloon and Green, 1987) or fertile MORB mantle (Pearce and Parkinson, 1993).

\section{OVERALL LITHOLOGICAL AND BULK CHEMICAL VARIABILITY OF LEG 153 ULTRAMAFIC, GABBROIC, AND TRANSITIONAL ROCKS}

The chemical and lithologic variability of serpentinized ultramafic and gabbroic samples from the suite of offset holes drilled during Leg 153 appears to be among the most diverse of any suite collected during a single leg that has sampled upper mantle and plutonic rocks of the oceanic lower crust (e.g., for comparisons with Hole 735B, see 


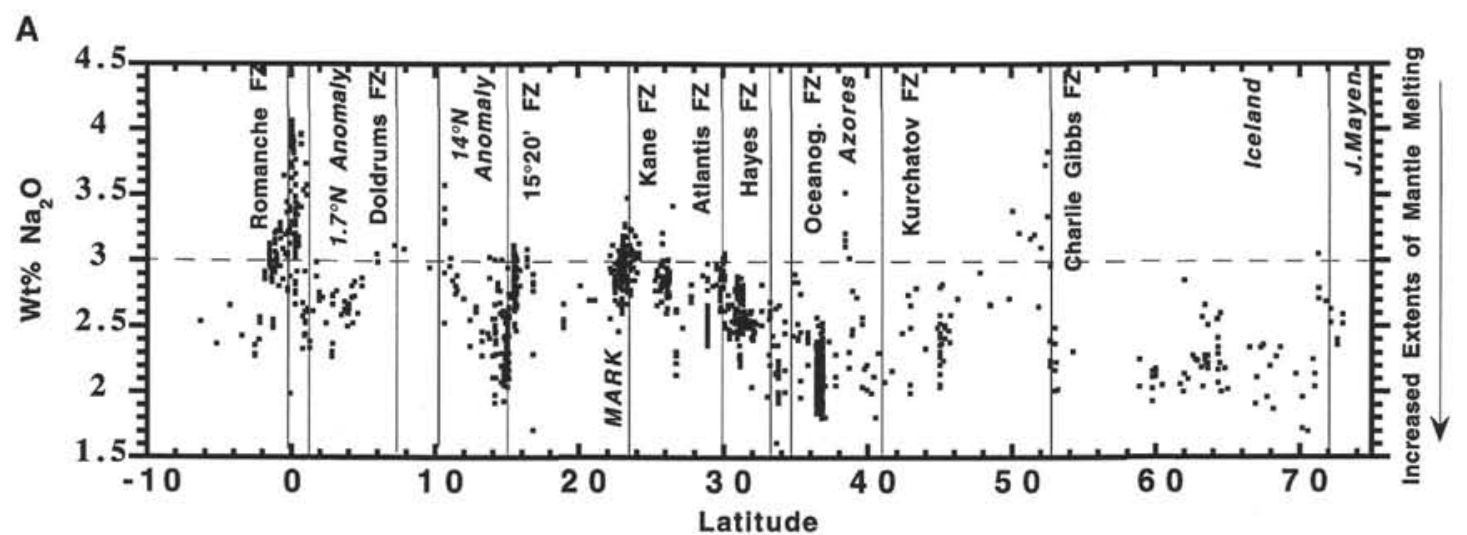

B

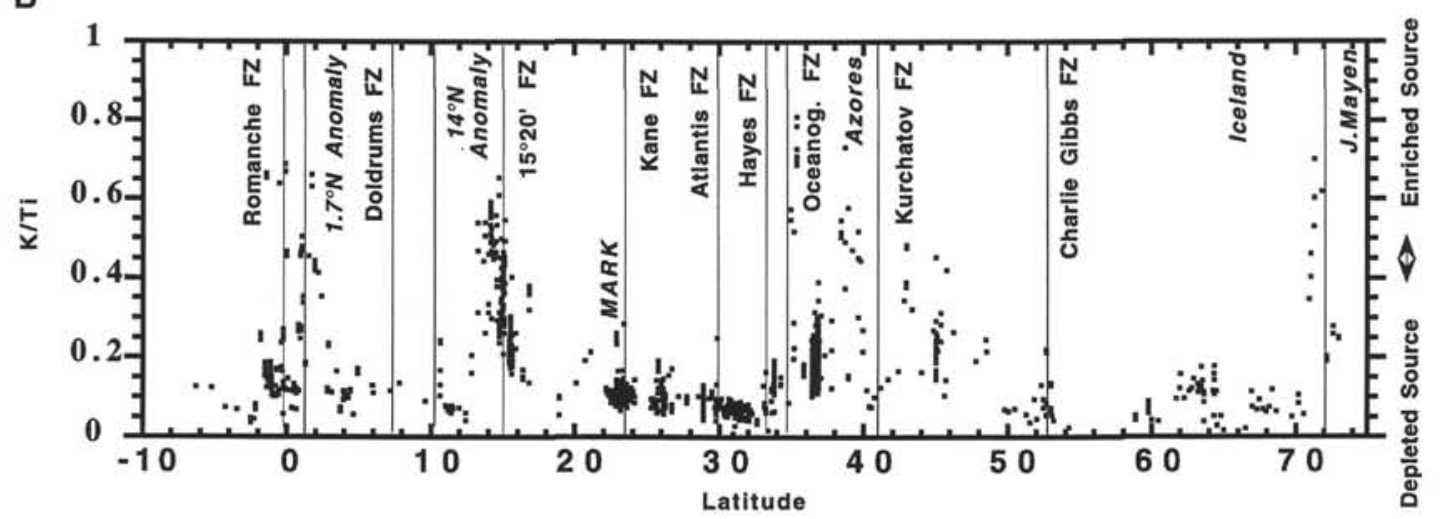

Figure 3. A. $\mathrm{Na}_{2} \mathrm{O}$ wt\% variations in glass along the Mid-Atlantic Ridge from $10^{\circ} \mathrm{S}$ to $70^{\circ} \mathrm{N}$. Increased extents of partial melting of the mantle are indicated by lower $\mathrm{Na}_{2} \mathrm{O}$ wt \% . The MARK region is just to the south of the Kane Fracture Zone (FZ) and is characterized by very high $\mathrm{Na}_{2} \mathrm{O}$ wt $\%$ content. B. $\mathrm{K} / \mathrm{Ti}$ ratios of basaltic glasses along the Mid-Atlantic Ridge from $10^{\circ} \mathrm{S}$ to $70^{\circ} \mathrm{N}$. Areas with high $\mathrm{K} / \mathrm{Ti}$ ratios indicate enriched mantle sources, and areas with low $\mathrm{K} / \mathrm{Ti}$ ratios indicate depleted mantle sources. The Azores hot spot and the $14^{\circ} 48^{\prime} \mathrm{N}$ anomaly show high $\mathrm{K} / \mathrm{Ti}$ ratios that are indications of enriched mantle sources. The MARK region between them represents typical normal depleted MORB with low K/Ti ratios. The MARK area south of the Kane Transform is characterized by low $\mathrm{K} / \mathrm{Ti}$, except for some elevated ratios south of the drill sites.

Dick, 1991). Lithologies recovered from Site 920 (Holes $920 \mathrm{~B}$ and 920D) from the ultramafic massif are dominantly variably serpentinized residual harzburgites, but include smaller amounts of variably serpentinized and altered lherzolite, clinopyroxenite, wehrlite, websterite, dunite, troctolite, olivine gabbro, gabbro, oxide gabbro, amphibolite, and high- $\mathrm{MgO}$ diabase that intrude into the residual ultramafic massif. In the following discussions, we refer to ultramafic rock types by their original protolith name, although most olivinerich rocks in the ultramafic sections are highly altered and serpentinized (Shipboard Scientific Party, 1995).

Mafic and silicic rocks recovered from Sites 921, 922, and 923 from the gabbroic massif include troctolite, olivine gabbro, gabbro, gabbro norite, oxide gabbro, quartz diorite, trondhjemite, and diabase. Combined with the basaltic samples previously recovered from the region, this range of recovered samples from the lower part of the oceanic crust and upper mantle essentially duplicates the lithologic range of most large well-exposed ophiolite complexes and the range of plutonic rocks recovered from the oceanic spreading centers and off-axis drill sites.

Recovery and lithologic units defined for each of the investigated holes drilled into the ultramafic and gabbroic massifs are shown in Figures $2 \mathrm{~A}$ and $2 \mathrm{~B}$, respectively. Recovery depths are recorded for each sample analyzed in Tables 1 through 5 . The geochemical and lithological diversity of Leg 153 samples is well displayed on the AFM diagram in Figure 6, where $\mathrm{A}=\mathrm{Na}_{2} \mathrm{O}+\mathrm{K}_{2} \mathrm{O}, \mathrm{F}=\mathrm{FeO}+\mathrm{Fe}_{2} \mathrm{O}_{3}$ $+\mathrm{MnO}$, and $\mathrm{M}=\mathrm{MgO}$. Bulk-rock geochemical data from Holes 920B and 920D drilled into the ultramafic massif, gabbroic, and silicic rocks from Sites 921, 922, and 923 drilled into the gabbroic mas- sif, and MARK basalts (Bryan et al., 1981; 1994) are displayed. The ultramafic samples plot along the $\mathrm{MgO}-\left(\mathrm{FeO}+\mathrm{Fe}_{2} \mathrm{O}_{3}+\mathrm{MnO}\right)$ join, and show a range from $\mathrm{MgO}$-rich harzburgites that are the residual products of mantle melting to more $\mathrm{FeO}$-rich wehrlites and pyroxenites that appear to be magmatic in origin. Gabbroic rocks show a strong iron-enrichment trend parallel to the linear trend of basalt glass data from the MARK area (Bryan et al., 1981; 1994), but displaced towards the more $\mathrm{MgO}$-rich apex as expected from cumulate assemblages in equilibrium with basalts.

The variation in bulk-rock $\mathrm{Mg} \#(100 \times \mathrm{Mg} /[\mathrm{Mg}+\mathrm{Fe} *])$ vs. $\mathrm{MgO} /$ $\mathrm{SiO}_{2}$ for Leg 153 gabbroic and ultramafic samples is displayed (Fig. 7A) to directly compare their lithologies and chemistries with the upper mantle and plutonic samples from a typical ophiolite complex like the Bay of Islands Complex (Casey et al., 1981) (Fig. 7B). The bulk-rock chemistry of ultramafic and mafic rocks from Hole 920B and 920D samples from the ultramafic massif are distinguished from the mafic plutonic samples from Sites 921, 922, and 923 from the gabbroic massif to allow direct comparisons. Bulk-rock $\mathrm{Mg \#} \mathrm{closely}$ reflects the $\mathrm{Mg} \#$ of mafic phases within the samples, and $\mathrm{MgO} / \mathrm{SiO}_{2}$ reflects the relative modal abundances of olivine, pyroxenes, and plagioclase. Mafic rocks sampled from both the gabbroic massif and the ultramafic massif show a similar wide compositional range in terms of their Mg\#. The bulk-rock chemistries of plutonic mafic and ultramafic cumulate rocks and residual mantle rocks from the Bay of Islands Ophiolite Complex (BOIC) (Fig. 7B) depict a similar compositional range in $\mathrm{Mg \#}$ and sample distribution to that of Leg 153.

In these plots, $\mathrm{MgO} / \mathrm{SiO}_{2}$ ratios are related to the mineral mode (i.e., the percent [olivine + clinopyroxene]/plagioclase). The $\mathrm{MgO}$ / 

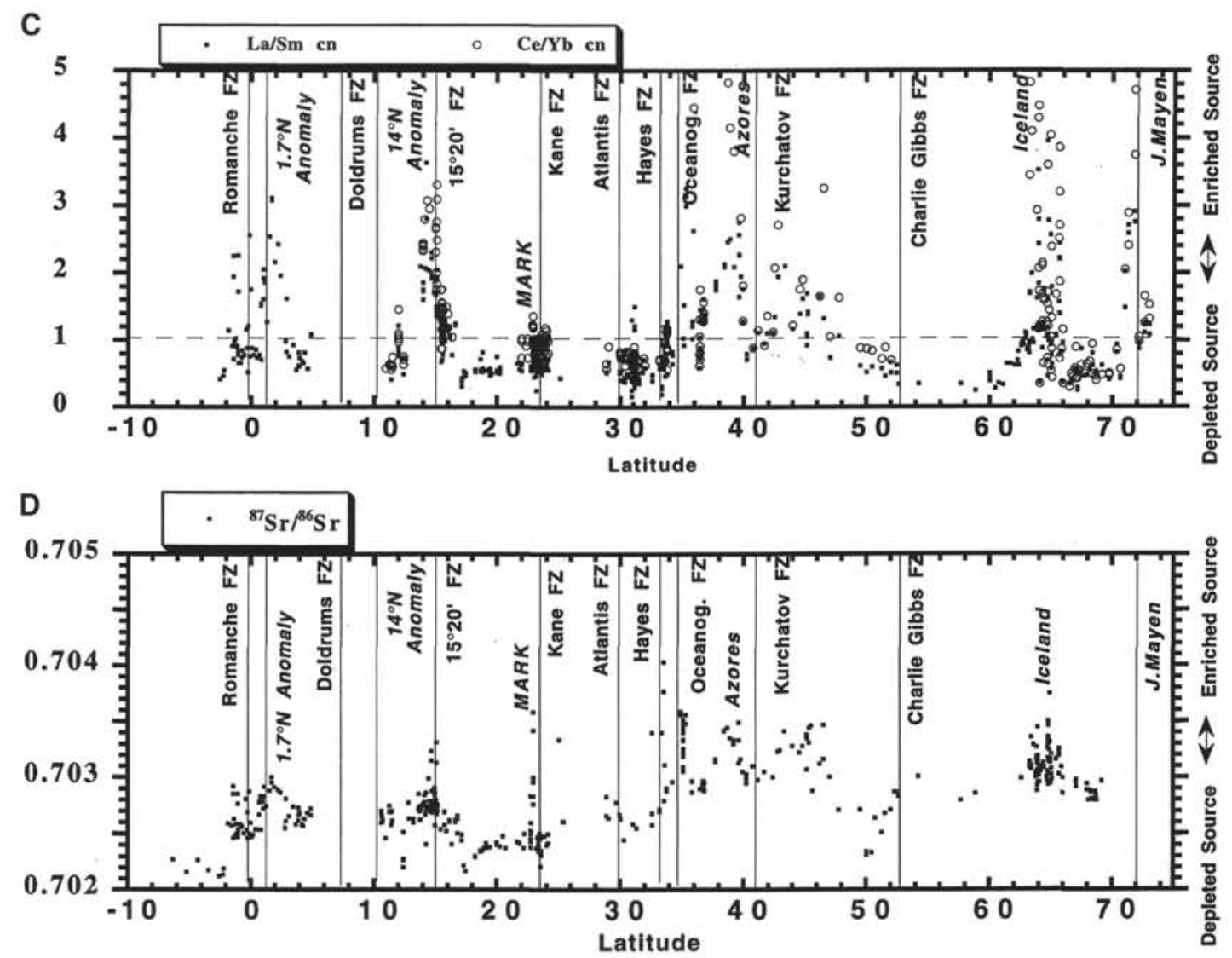

Figure 3 (continued). C. $\mathrm{Ce} / \mathrm{Yb}$ and $\mathrm{La} / \mathrm{Sm}$ variations in basalt along the Mid-Atlantic Ridge from $10^{\circ} \mathrm{S}$ to $70^{\circ} \mathrm{N}$. The MARK region is characterized by depleted N-type LREE/HREE and LREE/MREE ratios typical of N-MORB. D. ${ }^{87} \mathrm{Sr} /{ }^{86} \mathrm{Sr}$ isotopic ratios from $10^{\circ} \mathrm{S}$ to $70^{\circ} \mathrm{N}$ along the Mid-Atlantic Ridge. $14^{\circ} \mathrm{N}$, Azores, and Iceland are characterized by high ${ }^{87} \mathrm{Sr} /{ }^{86} \mathrm{Sr}$ ratios. Most of the MARK region basalts have low ${ }^{87} \mathrm{Sr} /{ }^{86} \mathrm{Sr}$. Several samples also show enhanced ${ }^{87} \mathrm{Sr} /{ }^{86} \mathrm{Sr}$ ratios that may be a result of seawater alteration. The North Atlantic MORB glass, trace-element, and isotopic data are compiled from White and Schilling (1978), Dupré and Allègre (1980), Sun (1980), Cohen and O'Nions (1982), Machado et al. (1982), Meyer et al. (1989), Ito et al. (1987), Shirey et al. (1987), Mertz et al. (1991), Hemond et al. (1993), Dosso et al. (1991; 1993), Bougault et al. (1988), Smith et al. (1991; 1995), S. Smith and J.F. Casey (unpubl. data), the Smithsonian catalog of glass analyses (Melson and O'Hearn, 1986), Sigurdsson (1981), Bryan et al. (1981; 1994), Stakes et al. (1984), Xia (1995), Schilling (1973), and Schilling et al. (1983; 1995).

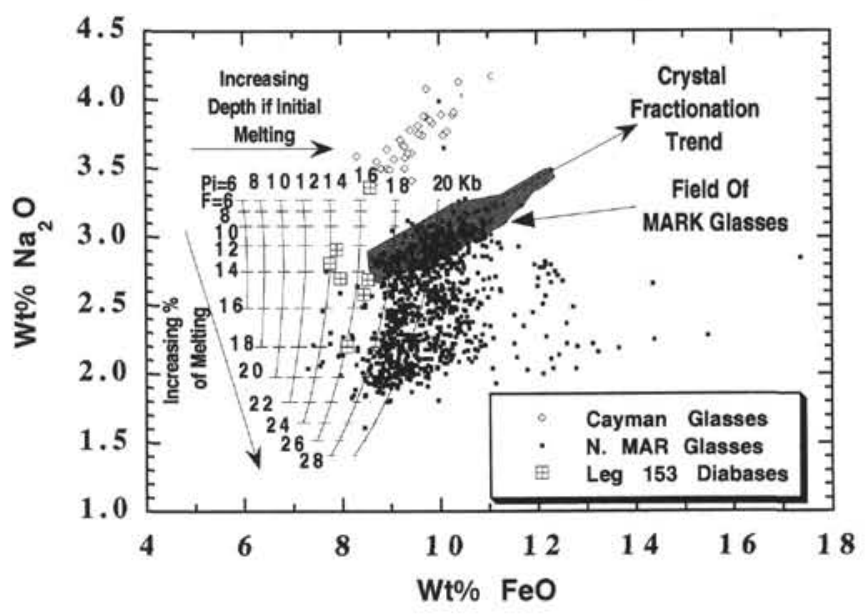

Figure 4. $\mathrm{Na}_{2} \mathrm{O}$ wt\% vs. $\mathrm{FeO}$ wt $\%$ of basaltic glasses from the North Atlantic and the Cayman Trough. Data sources are listed in Figure 2. Shown on the right are theoretical calculations of how $\mathrm{Na}_{2} \mathrm{O}$ and $\mathrm{FeO}$ would vary with respect to extent of melting $(F)$ and initial depth of partial melting $(P i)$ in response to decompression of an upwelling mantle (Niu and Batiza, 1991). The field from the MARK glasses is shown by the shaded pattern. Leg 153 altered diabases are also shown. In addition, data from glasses from the northern Mid-Atlantic Ridge and the Cayman Trough are plotted for reference. (Data sources as in Fig. 3.)
$\mathrm{SiO}_{2}$ ratio in olivine is the largest, and decreases progressively in the direction olivine $>$ orthopyroxene $>$ clinopyroxene $>>$ plagioclase. Thus, increases in modal plagioclase act to depress the ratio. Ultramafic rocks, including harzburgites, dunites, pyroxenites, and wehrlites, have ratios in excess of 0.25 . Harzburgites and lherzolite have the highest ratios, whereas clinopyroxene-rich wehrlites and pyroxenites have lower ratios. Plagioclase-bearing gabbroic rocks dominantly have a $\mathrm{MgO} / \mathrm{SiO}_{2}$ ratio less than 0.25 , although a few samples of olivine-rich troctolites have ratios slightly in excess of 0.25 . If iron oxides are low or absent, $\mathrm{Mg \# s}$ of the whole rock in these plots can be regarded as a direct measure of how primitive the melts in equilibrium with various cumulate and residual mafic minerals were, keeping in mind that the $\mathrm{Mg} \#$ of olivine will be somewhat lower than the $\mathrm{Mg} \#$ of clinopyroxene in equilibrium with the same melt because of small differences in the $\mathrm{Fe}-\mathrm{Mg}$ exchange partition coefficients (Roeder and Emslie, 1970; Hess, 1992). Generally, the differences in $\mathrm{Mg} \# \mathrm{~s}$ for the mafic phases are less than $2 \%$ (pyroxene $>$ olivine). Thus, the bulk-rock Mg\#s track the primitiveness of the mafic phases in the rock very well, regardless of the their relative modal percentages. In peridotites and most gabbros, $\mathrm{Fe}$ oxides are generally a very small component of the primary assemblage, or they are totally absent. Except for the most fractionated oxide-bearing gabbros, oxides will not contribute to significant lowering of the $\mathrm{Mg \# s}$ below that of the mafic phases for the majority of the samples. Oxide-rich gabbros generally have $\mathrm{Mg \# s}$ lower than 55 .

Harzburgite compositions exhibit the highest bulk-rock $\mathrm{Mg} \# \mathrm{~s}$ (>90). Dunite, wehrlite, and pyroxenite compositions have Mg\#s that 
A
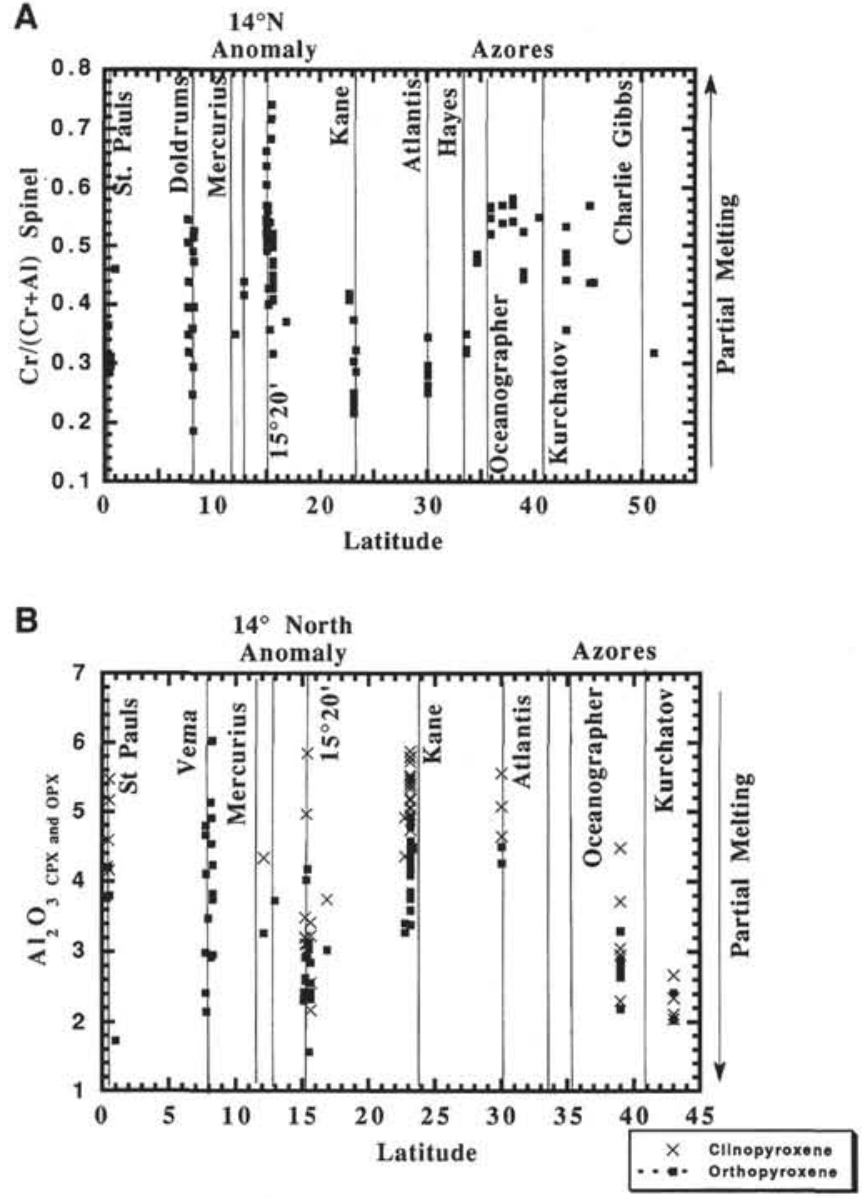

Figure 5. A. $\mathrm{Cr} /(\mathrm{Cr}+\mathrm{Al})$ in spinel variations from $0^{\circ}$ to $55^{\circ} \mathrm{N}$ along the MidAtlantic Ridge. The $\mathrm{Cr} \#$ is rather low in comparison to those for the $14^{\circ} \mathrm{N}$ and the Azores regions, suggesting low extents of melting. B. $\mathrm{Al}_{2} \mathrm{O}_{3}$ wt $\%$ in orthopyroxene or clinopyroxene from $0^{\circ}$ to $45^{\circ} \mathrm{N}$ along the Mid-Atlantic Ridge. $\mathrm{Al}_{2} \mathrm{O}_{3}$ contents in pyroxenes are high compared to those from the adjacent $14^{\circ} \mathrm{N}$ and the Azores regions. They also suggest that the MARK region is characterized by low degrees of mantle melting or depletion. Data sources are Komor et al. (1990), Juteau et al. (1990), Michael and Bonatti (1985), Bonatti and Michael (1989), Bonatti et al. (1993), Bonatti et al. (1992), Cannat (1993), Cannat and Casey (1995), and our unpublished data along the northern Mid-Atlantic Ridge (J.F. Casey and S. Silantyev, unpubl. data).

are generally in the range from 80 to 90 (see Table 2), reflecting the lower $\mathrm{Mg \# s}$ of pyroxenes and olivine in these rocks with respect to similar phases in residual harzburgites $(\mathrm{Mg} \# \mathrm{~s}>90)$. Fertile MORB mantle is typically regarded to have forsterite (Fo) values $>89-90$, so that residual products of mantle melting should generally have higher values than this. The lower Mg\#s of wehrlites, pyroxenites, and dunites confirm the nonresidual "cumulate" or "magmatic" origin of these rocks from Leg 153, as do similarly lower values in the BOIC samples. The term "cumulate" is not used here in the traditional sense of Wager and Brown (1968), but is simply used to describe any coarsely crystalline rock that represents mostly solid (>50\%) fractionally crystallized products (cumulus crystals) of a melt with from $0 \%$ to $50 \%$ solidified "intercumulus" trapped melt products. As used here, it does not necessarily infer or mandate any gravity crystal settling in the origin of the rock (Campbell, 1978; Irvine, 1979).

Although there are similarities between mantle and transition zone rock types and compositional ranges from the BOIC ophiolite and Leg 153 samples, there are some important differences in the range of $\mathrm{Mg \# s}$ of some of the intrusive plutonic rocks (including pyroxenites, wehrlites, gabbros, and oxide gabbros) within the residual mantle section of Site 920 . Some of these rocks show trends toward very fractionated compositions ( $\mathrm{Mg} \# \mathrm{~s}$ of $~ 30-57$ ). In comparison, basal ultramafic cumulates of the transition zone from the Bay of Islands and other ophiolites tend to be quite primitive in nature. Although they do have mineral compositions that are more evolved than the residual harzburgites, the extent of fractionation in the transition between residual ultramafic and mafic plutonic rocks is more limited within the ophiolite (Komor et al., 1985a; Elthon et al., 1984a; Elthon and Casey, 1985; J.F. Casey, unpubl. data). These compositional differences are also reflected in mineral chemistry of Leg 153 samples (see below). Thus, although lithologies and modal variation are similar in both the MARK samples and the BOIC, the bulk compositional ranges are somewhat different for the two data sets. Overall, the compositions of MARK ultramafic and gabbroic intrusive rocks within harzburgites of Hole 920B and 920D tend to be much more fractionated and evolved than their ophiolitic transition zone counterparts. Another important difference is that the distribution of various rock types is quite distinct. In the BOIC, transitional cumulate ultramafic dunites, wehrlites, clinopyroxenites, and websterites are located stratigraphically above the residual harzburgite (Casey et al., 1981; 1983 ) and are uncommon within the harzburgite, whereas MARK cumulate dunites, wehrlites, clinopyroxenites, websterites, and gabbroic rocks are distributed as intrusive rocks within the residual mantle section. Differences may reflect variable spreading rates and melt supply during crustal and upper mantle formation at slow-spreading centers. The colder and thicker lithospheric mechanical lid at slowspreading centers and the lower melt supply in the MARK region may act together to allow more extensive cooling and upper mantle fractionation of basaltic melts. This leads to the intermediate transitional compositions (wehrlites, dunites, lherzolite, pyroxenites, and gabbros) with $\mathrm{Mg} \#$ s between 90 and 80 , as well as the highly fractionated gabbroic and oxide gabbros (Mg\#s between 89 and 30). The products of solidification or reaction of these melts with the harzburgite wallrock are dispersed throughout the residual mantle rather than concentrated in a transition zone between the gabbroic unit and a residual mantle unit as observed in ophiolites with full classical stratigraphies (e.g., Casey et al., 1981; Pallister and Hopson, 1981; Nicolas, 1986).

Girardeau and Mercier (1992) and Girardeau and Francheteau (1993) have recently reviewed other peridotite drill sites where similar intrusive rocks have been noted, and reach the conclusion that such intrusive ultramafic and mafic rocks could form a substantial volume of the uppermost oceanic mantle. Moderate-pressure fractionation has also been suggested for the MARK region (Tormey et al., 1987), based on experimental investigations of MARK basalts. Moderate-pressure fractionation may be consistent with a thick mechanical lithosphere in the MARK area where mantle-derived melts can fractionate and evolve before reaching crustal levels.

Dick (1989) pointed out that dunites, wehrlites, and pyroxenites that are typical of ophiolite complex transition zones are generally absent from dredge collections of slow-spreading slipping transforms. MORB basalts and mineral assemblages of oceanic gabbros are generally too fractionated to be in equilibrium with mantle-derived melts, and the lack of primitive dunite, pyroxenite, wehrlite, and gabbroic rocks represents an important compositional and lithologic gap in oceanic suites. To date, this gap has not been satisfactorily explained. The ability to sample intact peridotite core on Leg 153 away from a major transform and to identify these intermediate (or typical transition zone) compositions and even more fractionated compositions dispersed throughout the mantle section in the MARK region may, in part, solve the dilemma regarding the lack of intermediate compositions in the oceanic realm. These rocks may be undersampled at fracture zones because they are disguised by mantle veining or they may be present only away form fracture zones towards the center of the spreading segments where magma delivery is focused. Presumably, melt withdrawal is likely to predominate mantle sections near major transform boundaries, whereas focused flow and melt delivery at the center of a segment is likely to promote formation of transitional ultramafic and mafic rocks and a secondary veining of 
Table 1. Bulk-rock major- and trace-element geochemistry of Holes $920 \mathrm{~A}$ and $920 \mathrm{~B}$ residual mantle and plutonic ultramafic and mafic samples.

\begin{tabular}{|c|c|c|c|c|c|c|c|c|c|c|c|c|c|c|}
\hline Hole: & $920 \mathrm{~A}$ & $920 \mathrm{~B}$ & $920 \mathrm{~B}$ & $920 \mathrm{~B}$ & $920 \mathrm{~B}$ & $920 \mathrm{~B}$ & $920 \mathrm{~B}$ & $920 \mathrm{~B}$ & $920 \mathrm{~B}$ & $920 \mathrm{~B}$ & $920 \mathrm{~B}$ & $920 \mathrm{~B}$ & $920 \mathrm{~B}$ & $920 \mathrm{~B}$ \\
\hline Core, section, interval $(\mathrm{cm})$ : & $1 \mathrm{~W}-1,73-77$ & $2 \mathrm{R}-1,17-20$ & $3 R-1,84-90$ & $3 \mathrm{R}-2,98-101$ & $4 R-1,60-63$ & SR-1, 100-106 & $5 R-3,86-87$ & $6 \mathrm{R}-3,20-26$ & $7 \mathrm{R}-1,52-58$ & $7 \mathrm{R}-2,114-11$ & $R-3,15-18$ & $8 R-2,70-77$ & $8 \mathrm{R}-3,115-120$ & $9 R-2,36-43$ \\
\hline Depth (mbsf): & 0.73 & 14.17 & 24.34 & 25.98 & 33.80 & 43.70 & 46.48 & 55.05 & 61.52 & 63.56 & 63.98 & 72.38 & 74.17 & 81.55 \\
\hline $\begin{array}{l}\text { Laboratory } \\
\text { (major, trace): }\end{array}$ & $\begin{array}{l}\text { Shipboard } \\
\text { XRF }\end{array}$ & $\begin{array}{l}\text { Shipboard } \\
\text { XRF }\end{array}$ & ICP-AES-UH & $\begin{array}{l}\text { Shipboard } \\
\text { XRF }\end{array}$ & $\begin{array}{c}\text { Shipboard } \\
\text { XRF }\end{array}$ & ICP-AES-UH & $\begin{array}{l}\text { Shipboard } \\
\text { XRF }\end{array}$ & $\begin{array}{l}\text { Shipboard } \\
\text { XRF }\end{array}$ & $\begin{array}{c}\text { Shipboard } \\
\text { XRF }\end{array}$ & $\begin{array}{c}\text { Shipboard } \\
\text { XRF }\end{array}$ & $\begin{array}{c}\text { Shipboard } \\
\text { XRF }\end{array}$ & $\begin{array}{c}\text { Shipboard } \\
\text { XRF }\end{array}$ & $\begin{array}{c}\text { Shipboard } \\
\text { XRF }\end{array}$ & $\begin{array}{c}\text { Shipboard } \\
\text { XRF }\end{array}$ \\
\hline Lithology: & Harzburgite & Harzburgite & Harzburgite & Harzburgite & Harzburgite & Harzburgite & Harzburgite & Harzburgite & Harzburgite & Harzburgite & Harzburgite & Harzburgite & Harzburgite & Harzburgite \\
\hline \multicolumn{15}{|l|}{ Major elements (wt\%) } \\
\hline $\mathrm{SiO}_{2}$ & 44.61 & 44.14 & 44.90 & 43.74 & 44.97 & 44.21 & 44.29 & 46.16 & 44.49 & 44.02 & 44.86 & 44.22 & 44.61 & 43.87 \\
\hline $\mathrm{TiO}_{2}^{2}$ & 0.02 & 0.02 & 0.02 & 0.02 & 0.02 & 0.03 & 0.02 & 0.01 & 0.01 & 0.02 & 0.02 & 0.01 & 0.02 & 0.01 \\
\hline $\mathrm{Al}_{2} \mathrm{O}_{3}$ & 1.14 & 1.41 & 1.32 & 1.29 & 1.83 & 1.56 & 1.52 & 1.56 & 1.44 & 1.24 & 1.39 & 1.22 & 1.33 & 1.06 \\
\hline $\mathrm{Fe}_{2} \mathrm{O}_{3}$ & 9.12 & 9.67 & 9.37 & 9.27 & 9.12 & 9.04 & 9.71 & 9.45 & 9.24 & 9.21 & 8.72 & 9.58 & 9.03 & 9.42 \\
\hline $\mathrm{MnO}$ & 0.14 & 0.13 & 0.14 & 0.14 & 0.13 & 0.12 & 0.14 & 0.12 & 0.11 & 0.14 & 0.14 & 0.12 & 0.13 & 0.12 \\
\hline $\mathrm{MgO}$ & 44.60 & 44.01 & 44.12 & 44.72 & 42.82 & 44.85 & 44.37 & 43.34 & 44.17 & 46.21 & 44.12 & 44.49 & 44.41 & 45.00 \\
\hline $\mathrm{CaO}$ & 0.01 & 0.10 & 0.00 & 0.01 & 0.42 & 0.00 & 0.18 & 0.33 & 0.21 & 0.06 & 0.33 & 0.06 & 0.13 & 0.14 \\
\hline $\mathrm{Na}_{2} \mathrm{O}$ & 0.00 & 0.00 & 0.02 & 0.00 & 0.00 & 0.00 & 0.00 & 0.00 & 0.00 & 0.00 & 0.00 & 0.00 & 0.00 & 0.00 \\
\hline $\mathrm{K}_{2} \mathrm{O}$ & 0.00 & 0.00 & 0.00 & 0.00 & 0.01 & 0.00 & 0.00 & 0.00 & 0.00 & 0.00 & 0.00 & 0.01 & 0.00 & 0.00 \\
\hline $\mathrm{P}_{2} \mathrm{O}_{5}$ & 0.00 & 0.00 & 0.00 & 0.00 & 0.00 & 0.00 & 0.00 & 0.00 & 0.00 & 0.00 & 0.00 & 0.00 & 0.00 & 0.00 \\
\hline $\mathrm{Cr}_{2} \mathrm{O}_{3}$ & 0.31 & 0.37 & 0.39 & 0.38 & 0.54 & 0.49 & 0.40 & 0.39 & 0.39 & 0.38 & 0.43 & 0.39 & 0.41 & 0.38 \\
\hline $\mathrm{NiO}$ & 0.33 & 0.28 & 0.32 & 0.35 & 0.32 & 0.34 & 0.32 & 0.27 & 0.33 & 0.34 & 0.32 & 0.34 & 0.33 & 0.35 \\
\hline Total & 100.29 & 100.13 & 100.60 & 99.92 & 100.19 & 100.63 & 100.96 & 101.63 & 100.39 & 101.62 & 100.33 & 100.44 & 100.40 & 100.35 \\
\hline LOI & 12.78 & 12.10 & 12.38 & 12.81 & 11.51 & 12.19 & 12.26 & 11.41 & 11.97 & 13.22 & 12.04 & 12.51 & 12.75 & 12.41 \\
\hline $\mathrm{CO}_{2}$ & 0.03 & 0.24 & & 0.19 & 0.41 & & 0.08 & 0.31 & 0.27 & 0 & 0.34 & 0.3 & 0.31 & 0.3 \\
\hline $\mathrm{H}_{2} \mathrm{O}$ & 13.1 & 12.7 & & 16 & 11.1 & & 15.4 & 12.4 & 12.6 & 13.4 & 11.6 & 14.6 & 14.4 & 13.2 \\
\hline FeOT & 8.21 & 8.70 & 8.43 & 8.34 & 8.21 & 8.13 & 8.74 & 8.50 & 8.31 & 8.29 & 7.85 & 8.62 & 8.13 & 8.48 \\
\hline \multicolumn{15}{|l|}{ Trace elements (ppm) } \\
\hline $\mathrm{V}$ & 48 & 43 & 78 & 50 & 63 & 88 & 50 & 45 & 51 & 50 & 55 & 50 & 54 & 44 \\
\hline $\mathrm{Ti}$ & 120 & 120 & 120 & 120 & 120 & 180 & 120 & 60 & 60 & 120 & 120 & 60 & 120 & 60 \\
\hline $\mathrm{Cr}$ & 2147 & 2510 & 2648 & 2576 & 3713 & 3335 & 2770 & 2646 & 2681 & 2631 & 2967 & 2679 & 2815 & 2615 \\
\hline $\mathrm{Ni}$ & 2609 & 2198 & 2536 & 2757 & 2540 & 2645 & 2526 & 2127 & 2565 & 2638 & 2529 & 2641 & 2624 & 2772 \\
\hline $\mathrm{Sr}$ & 8 & 9 & 10 & 8 & 18 & 10 & 8 & 9 & 8 & 8 & 8 & 8 & 8 & 8 \\
\hline $\mathrm{Zr}$ & 5 & 4 & -1 & 5 & 5 & 3 & 5 & 6 & 5 & 4 & 4 & 4 & 4 & 4 \\
\hline $\mathrm{Ba}$ & 0 & 0 & 18 & 0 & 0 & 19 & 0 & 0 & 0 & 0 & 0 & 0 & 0 & 0 \\
\hline \multirow{2}{*}{\multicolumn{15}{|c|}{$\begin{array}{l}\mathrm{Co} \\
\mathrm{Cu}\end{array}$}} \\
\hline & & & & & & & & & & & & & & \\
\hline \multicolumn{15}{|l|}{$\mathrm{Zn}$} \\
\hline $\begin{array}{l}\mathrm{Sc} \\
\mathrm{Y}\end{array}$ & & & & & & $\begin{array}{r}25.85 \\
0.96\end{array}$ & & & & & & & & \\
\hline $\mathrm{Y}$ & & & & & & 0.96 & & & & & & & & \\
\hline \multicolumn{15}{|l|}{ Rare earths (ppm) } \\
\hline $\mathrm{La}$ & & & & & & 0.63 & & & & & & & & \\
\hline $\mathrm{Ce}$ & & & & & & 1.34 & & & & & & & & \\
\hline $\mathrm{Nd}$ & & & & & & 0.25 & & & & & & & & \\
\hline $\mathrm{Sm}$ & & & & & & 0.26 & & & & & & & & \\
\hline Eu & & & & & & 0.27 & & & & & & & & \\
\hline Gd & & & & & & 1.15 & & & & & & & & \\
\hline Dy & & & & & & 0.04 & & & & & & & & \\
\hline $\mathrm{Er}$ & & & & & & 0.21 & & & & & & & & \\
\hline $\begin{array}{l}\mathrm{Yb} \\
\mathrm{Lu}\end{array}$ & & & & & & $\begin{array}{l}0.23 \\
0.03\end{array}$ & & & & & & & & \\
\hline
\end{tabular}

Notes: $\mathrm{UH}=$ University of Houston. $\mathrm{FeOT}=$ all iron as $\mathrm{FeO}$. 
Table 1 (continued).

\begin{tabular}{|c|c|c|c|c|c|c|c|c|c|c|c|c|c|}
\hline Hole: & $920 \mathrm{~B}$ & $920 \mathrm{~B}$ & $920 \mathrm{~B}$ & $920 \mathrm{~B}$ & $920 \mathrm{~B}$ & $920 \mathrm{~B}$ & $920 \mathrm{~B}$ & $920 \mathrm{~B}$ & $920 \mathrm{~B}$ & $920 \mathrm{~B}$ & $920 \mathrm{~B}$ & $920 \mathrm{~B}$ & $920 \mathrm{~B}$ \\
\hline Core, section, interval $(\mathrm{cm})$ : & $9 R-2,84-91$ & $9 R-3,55-57$ & $9 R-4,17-20$ & $10 \mathrm{R}-1,17-23$ & $10 \mathrm{R}-3,0-5^{*}$ & $10 \mathrm{R}-3,5-21$ & $10 \mathrm{R}-3,136-143$ & $10 R-4,139-143$ & $11 \mathrm{R}-1,0-5$ & $11 R-1,119-125$ & $11 \mathrm{R}-1,110-119 \mathrm{~A}$ & A $11 R-1,110-119 B$ & $12 R-1,23-28$ \\
\hline Depth (mbsf): & 82.00 & 83.20 & 84.29 & 89.30 & 91.87 & 92.02 & 98.23 & 94.70 & 98.50 & 99.69 & 99.69 & 99.60 & 107.93 \\
\hline $\begin{array}{l}\text { Laboratory } \\
\text { (major, trace): }\end{array}$ & $\begin{array}{c}\text { Shipboard } \\
\text { XRF }\end{array}$ & $\begin{array}{c}\text { Shipboard } \\
\text { XRF }\end{array}$ & ICP-AES-UH & $\begin{array}{l}\text { Shipboard } \\
\text { XRF }\end{array}$ & ICP-AES-UH & ICP-AES-UH & ICP-AES-UH & $\begin{array}{c}\text { Shipboard } \\
\text { XRF }\end{array}$ & ICP-AES-UH & $\begin{array}{c}\text { Shipboard } \\
\text { XRF }\end{array}$ & ICP-AES-UH & ICP-AES-UH & ICP-AES-UH \\
\hline Lithology: & Harzburgite & Diabase & Diabase & Harzburgite & $\begin{array}{c}\text { Metagabbro } \\
\text { vein }\end{array}$ & Harzburgite & Harzburgite & Harzburgite & Harzburgite & Harzburgite & Harzburgite & Harz/Gabbro dike & $\begin{array}{c}\text { Harzburgite- } \\
\text { Lehrz }\end{array}$ \\
\hline \multicolumn{14}{|l|}{ Major elements (wt\%) } \\
\hline $\mathrm{SiO}_{2}$ & 44.71 & 46.64 & 48.25 & 43.97 & 46.44 & 44.96 & 45.01 & 43.88 & 45.76 & 44.30 & 44.10 & 43.24 & 44.23 \\
\hline $\mathrm{TiO}_{2}^{2}$ & 0.02 & 1.04 & 0.91 & 0.03 & 0.20 & 0.03 & 0.03 & 0.02 & 0.02 & 0.02 & 0.03 & 0.23 & 0.02 \\
\hline $\mathrm{Al}_{2} \mathrm{O}_{3}$ & 1.42 & 18.05 & 17.47 & 1.51 & 8.92 & 1.30 & 1.61 & 1.33 & 1.31 & 1.48 & 1.53 & 4.77 & 1.59 \\
\hline $\mathrm{Fe}_{2} \mathrm{O}_{3}$ & 9.10 & 9.00 & 8.62 & 9.93 & 3.28 & 9.45 & 9.10 & 9.50 & 9.13 & 9.20 & 10.10 & 12.23 & 9.03 \\
\hline $\mathrm{MnO}$ & 0.13 & 0.25 & 0.16 & 0.13 & 0.21 & 0.11 & 0.14 & 0.13 & 0.11 & 0.13 & 0.13 & 0.22 & 0.13 \\
\hline $\mathrm{MgO}$ & 44.37 & 13.03 & 12.14 & 43.95 & 16.07 & 43.07 & 43.05 & 45.33 & 43.63 & 43.44 & 40.96 & 32.76 & 43.72 \\
\hline $\mathrm{CaO}$ & 0.04 & 8.92 & 9.58 & 0.08 & 23.77 & 0.23 & 0.74 & 0.23 & 0.51 & 0.37 & 0.85 & 7.18 & 1.17 \\
\hline $\mathrm{Na}_{2} \mathrm{O}$ & 0.00 & 2.20 & 2.81 & 0.00 & 0.08 & 0.00 & 0.00 & 0.00 & 0.02 & 0.00 & 0.00 & 0.06 & 0.01 \\
\hline $\mathrm{K}_{2} \mathrm{O}$ & 0.00 & 0.01 & 0.02 & 0.00 & 0.00 & 0.00 & 0.00 & 0.00 & 0.01 & 0.00 & 0.00 & 0.00 & 0.00 \\
\hline $\mathrm{P}_{2} \mathrm{O}_{5}$ & 0.00 & 0.05 & 0.05 & 0.00 & 0.02 & 0.00 & 0.00 & 0.00 & 0.01 & 0.00 & 0.00 & 0.04 & 0.02 \\
\hline $\mathrm{Cr}_{2} \mathrm{O}_{3}$ & 0.42 & 0.06 & 0.07 & 0.33 & 0.06 & 0.36 & 0.49 & 0.33 & 0.33 & 0.49 & 0.38 & 0.30 & 0.44 \\
\hline $\mathrm{NiO}^{3}$ & 0.32 & 0.03 & 0.03 & 0.27 & 0.05 & 0.29 & 0.31 & 0.35 & 0.28 & 0.34 & 0.31 & 0.22 & 0.30 \\
\hline Total & 100.54 & 99.28 & 100.11 & 100.19 & 99.10 & 99.80 & 100.49 & 101.11 & 101.12 & 99.77 & 98.39 & 101.25 & 100.66 \\
\hline LOI & 12.77 & 4.29 & 3.61 & 12.23 & 4.05 & 11.63 & 9.76 & 13.82 & 4.05 & 11.25 & 9.71 & 8.36 & 10.02 \\
\hline $\begin{array}{l}\mathrm{CO}_{2} \\
\mathrm{H}_{2} \mathrm{O}\end{array}$ & $\begin{array}{c}0.13 \\
13.1\end{array}$ & $\begin{array}{l}0 \\
5.87\end{array}$ & & $\begin{array}{r}0.3 \\
15.4\end{array}$ & & & & $\begin{array}{l}0.03 \\
13.1\end{array}$ & & $\begin{array}{l}0.26 \\
12.3\end{array}$ & & & \\
\hline $\begin{array}{l}\mathrm{H}_{2} \mathrm{O} \\
\mathrm{FeOT}\end{array}$ & 8.19 & 8.10 & 7.76 & $\begin{array}{l}18.4 \\
8.94\end{array}$ & 2.95 & 8.50 & 8.19 & 8.55 & 8.22 & $\begin{array}{l}1.3 .3 \\
8.28\end{array}$ & 9.09 & 11.00 & 8.13 \\
\hline \multicolumn{14}{|l|}{ Trace elements (ppm) } \\
\hline V & 57 & 214 & 184 & 44 & 144 & 79 & 66 & 48 & 60 & 58 & 230 & 130 & 57 \\
\hline $\mathrm{Ti}$ & 120 & 6235 & 5455 & 180 & 1199 & 180 & 180 & 120 & 115 & 120 & 180 & 1379 & 132 \\
\hline $\mathrm{Cr}$ & 2897 & 391 & 469 & 2226 & 381 & 2429 & 3385 & 2270 & 2279 & 3349 & 2585 & 2065 & 2977 \\
\hline $\mathrm{Ni}$ & 2547 & 228 & 273 & 2092 & 404 & 2315 & 2455 & 2784 & 2203 & 2647 & 2448 & 1727 & 2346 \\
\hline $\mathrm{Sr}$ & 8 & 190 & 340 & 9 & 21 & 12 & 8 & 8 & 4 & 8 & 7 & 46 & 3 \\
\hline $\mathrm{Zr}$ & 4 & 70 & 69 & 5 & 16 & 5 & & 4 & 5 & 4 & 7 & 26 & 5 \\
\hline $\mathrm{Ba}$ & 0 & 1 & 55 & 0 & 17 & 20 & 16 & 0 & 9 & 0 & 12 & 13 & 10 \\
\hline \multirow{2}{*}{\multicolumn{14}{|c|}{$\begin{array}{l}\mathrm{Co} \\
\mathrm{Cu}\end{array}$}} \\
\hline & & & & & & & \multicolumn{7}{|c|}{$\mathrm{Zn}$} \\
\hline $\mathrm{Sc}$ & & & 30.19 & 29.18 & & & & & & & & & \\
\hline $\mathrm{Y}$ & & & 21.47 & 6.31 & & & & & & & & & \\
\hline \multicolumn{14}{|l|}{ Rare earths (ppm) } \\
\hline $\mathrm{La}$ & & & 2.06 & & 0.18 & & & & & & & & \\
\hline $\mathrm{Ce}$ & & & 7.30 & & 0.83 & & & & & & & & \\
\hline $\mathrm{Nd}$ & & & 6.15 & & 0.87 & & & & & & & & \\
\hline $\mathrm{Sm}$ & & & 1.91 & & 0.37 & & & & & & & & \\
\hline Eu & & & 0.76 & & 0.30 & & & & & & & & \\
\hline Gd & & & 2.44 & & 0.63 & & & & & & & & \\
\hline Dy & & & 3.31 & & 1.00 & & & & & & & & \\
\hline $\mathrm{Er}$ & & & 2.11 & & 0.70 & & & & & & & & \\
\hline $\mathrm{Yb}$ & & & 2.01 & & 0.57 & & & & & & & & \\
\hline Lu & & & 0.29 & & & & & & & & & & \\
\hline
\end{tabular}


Table 1 (continued).

\begin{tabular}{|c|c|c|c|c|c|c|c|c|c|c|}
\hline Hole: & $920 \mathrm{~B}$ & $920 \mathrm{~B}$ & $920 \mathrm{~B}$ & $920 \mathrm{~B}$ & $920 \mathrm{~B}$ & $920 \mathrm{~B}$ & $920 \mathrm{~B}$ & $920 \mathrm{~B}$ & $920 \mathrm{~B}$ & $920 \mathrm{~B}$ \\
\hline Core, section, interval $(\mathrm{cm})$ : & $12 \mathrm{R}-2,77-84$ & $12 \mathrm{R}-3,100-106$ & $12 \mathrm{R}-5,10-17$ & $12 \mathrm{R}-5,32-40$ & $12 R-5,128-133$ & $13 \mathrm{R}-1,33-38$ & $13 R-3,15-21$ & $13 R-3,94-103$ & $13 R-3,129-1$ & $13 R-4,23-26$ \\
\hline Depth (mbsf): & 109.92 & 111.65 & 113.10 & 113.32 & 114.28 & 117.53 & 120.04 & 120.83 & 121.18 & 121.51 \\
\hline $\begin{array}{l}\text { Laboratory } \\
\text { (major, trace): }\end{array}$ & ICP-AES-UH & ICP-AES-UH & $\begin{array}{l}\text { Shipboard } \\
\text { XRF }\end{array}$ & ICP-AES-UH & ICP-AES-UH & $\begin{array}{c}\text { Shipboard } \\
\text { XRF }\end{array}$ & ICP-AES-UH & ICP-AES-UH & $\begin{array}{c}\text { Shipboard } \\
\text { XRF }\end{array}$ & $\begin{array}{l}\text { Shipboard } \\
\text { XRF }\end{array}$ \\
\hline Lithology: & Harzburgite & Harzburgite & Harzburgite & Harzburgite & Harzburgite & Harzburgite & Harzburgite & Harzburgite & Ferrogabbro & $\begin{array}{c}\text { Gneissic } \\
\text { amphibolite }\end{array}$ \\
\hline \multicolumn{11}{|l|}{ Major elements (wt\%) } \\
\hline $\mathrm{SiO}_{2}$ & 45.40 & 44.14 & 43.81 & 44.69 & 43.49 & 42.63 & 44.33 & 44.23 & 49.84 & 46.64 \\
\hline $\mathrm{TiO}_{2}$ & 0.02 & 0.02 & 0.02 & 0.02 & 0.02 & 0.02 & 0.02 & 0.03 & 2.89 & 1.06 \\
\hline $\mathrm{Al}_{2} \mathrm{O}_{3}$ & 1.57 & 1.25 & 1.40 & 1.43 & 1.35 & 1.27 & 1.34 & 1.30 & 16.93 & 16.13 \\
\hline $\mathrm{Fe}_{2} \mathrm{O}_{3}$ & 8.91 & 9.26 & 9.22 & 9.09 & 9.44 & 9.21 & 8.79 & 9.31 & 13.38 & 11.83 \\
\hline $\mathrm{MnO}$ & 0.13 & 0.13 & 0.14 & 0.13 & 0.13 & 0.13 & 0.14 & 0.18 & 0.19 & 0.19 \\
\hline $\mathrm{MgO}$ & 42.31 & 42.54 & 43.55 & 43.13 & 43.68 & 45.98 & 45.19 & 43.45 & 3.14 & \\
\hline $\mathrm{CaO}$ & 1.23 & 1.04 & 1.01 & 1.15 & 0.70 & 0.88 & 0.35 & 0.87 & 8.10 & 9.65 \\
\hline $\mathrm{Na}_{2} \mathrm{O}$ & 0.00 & 0.00 & 0.00 & 0.00 & 0.00 & 0.00 & 0.04 & 0.03 & 4.63 & 2.46 \\
\hline $\mathrm{K}_{2} \mathrm{O}$ & 0.00 & 0.00 & 0.00 & 0.00 & 0.00 & 0.00 & 0.02 & 0.02 & 0.09 & 0.04 \\
\hline $\mathrm{P}_{2} \mathrm{O}_{4}$ & 0.01 & 0.00 & 0.00 & 0.01 & 0.01 & 0.00 & 0.01 & 0.01 & 0.00 & 0.02 \\
\hline $\mathrm{Cr}_{2} \mathrm{O}_{3}$ & 0.43 & 0.42 & 0.40 & 0.42 & 0.46 & 0.38 & 0.44 & 0.59 & 0.01 & 0.05 \\
\hline $\mathrm{NiO}^{3}$ & 0.30 & 0.34 & 0.33 & 0.35 & 0.35 & 0.35 & 0.31 & 0.31 & 0.01 & 0.04 \\
\hline Total & 100.32 & 99.14 & 99.88 & 100.42 & 99.63 & 100.85 & 100.99 & 100.33 & 99.21 & 88.11 \\
\hline LOI & 11.07 & 12.50 & 11.86 & 12.28 & 12.59 & 12.60 & 11.85 & 12.02 & 1.26 & -0.39 \\
\hline $\mathrm{CO}_{2}$ & & & 0.52 & & & 0.33 & & & 0 & 0.08 \\
\hline $\mathrm{H}_{2} \mathrm{O}$ & & & 12.4 & & & 14.3 & & & 2.29 & 0.71 \\
\hline $\mathrm{FeOT}$ & 8.02 & 8.33 & 8.30 & 8.18 & 8.49 & 8.29 & 7.91 & 8.38 & 12.04 & 10.64 \\
\hline \multicolumn{11}{|l|}{ Trace elements (ppm) } \\
\hline $\mathrm{V}$ & 62 & 94 & 57 & 74 & 71 & 44 & 74 & 62 & 339 & 163 \\
\hline $\mathrm{Ti}$ & 120 & 120 & 120 & 120 & 120 & 120 & 147 & 162 & 17326 & 6355 \\
\hline $\mathrm{Cr}$ & 2966 & 2846 & 2749 & 2879 & 3164 & 2577 & 3014 & 4030 & 72 & 348 \\
\hline $\mathrm{Ni}$ & 2396 & 2676 & 2576 & 2741 & 2766 & 2762 & 2449 & 2442 & 60 & 322 \\
\hline $\mathrm{Sr}$ & 22 & 10 & 8 & 12 & 10 & 8 & 9 & 24 & 391 & 132 \\
\hline $\mathrm{Zr}$ & 7 & 2 & 4 & 6 & 8 & $\begin{array}{l}\circ \\
4\end{array}$ & 4 & 3 & 72 & 55 \\
\hline $\mathrm{Ba}$ & 15 & 18 & 0 & 18 & 14 & 0 & 17 & 13 & 23 & 12 \\
\hline Co & & & & & & & & & 67 & 76 \\
\hline $\mathrm{Cu}$ & & & & & & & & & 45 & 56 \\
\hline $\mathrm{Zn}$ & & & & & & & & & 149 & 75 \\
\hline $\mathrm{Sc}$ & & & & & & & & & 22.50 & \\
\hline $\mathrm{Y}$ & & & & & & & & & 29.71 & \\
\hline \multicolumn{11}{|l|}{ Rare earths (ppm) } \\
\hline $\mathrm{La}$ & & & & & & & & & 2.06 & \\
\hline $\mathrm{Ce}$ & & & & & & & & & 7.19 & \\
\hline $\mathrm{Nd}$ & & & & & & & & & 6.74 & \\
\hline $\mathrm{Sm}$ & & & & & & & & & 2.44 & \\
\hline Eu & & & & & & & & & 2.88 & \\
\hline Gd & & & & & & & & & 3.63 & \\
\hline Dy & & & & & & & & & 4.89 & \\
\hline $\mathrm{Er}$ & & & & & & & & & 3.15 & \\
\hline $\mathrm{Yb}$ & & & & & & & & & 3.01 & \\
\hline $\mathrm{Lu}$ & & & & & & & & & 0.49 & \\
\hline
\end{tabular}


Table 2. Bulk-rock major- and trace-element geochemistry of Hole 920D residual mantle and plutonic ultramafic and mafic samples.

\begin{tabular}{|c|c|c|c|c|c|c|c|c|c|c|c|c|c|}
\hline Hole: & 920D & 920D & 920D & 920D & 920D & 920D & 920D & 920D & 920D & 920D & 920D & 920D & 920D \\
\hline Core, section, interval $(\mathrm{cm})$ : & $2 \mathrm{R}-1,21-27$ & $2 \mathrm{R}-1,45-51$ & 2R-1, 133-138 & $3 R-1,38-41$ & $3 R-1,130-136$ & $4 \mathrm{R}-1,80-84$ & $4 \mathrm{R}-2,45-51$ & $4 R-2-97-100$ & $5 R-1,71-74$ & $5 R-2,13-19$ & $5 R-2,33-39$ & $5 R-4,0-5$ & $5 R-4,19-25$ \\
\hline Depth (mbsf): & 8.21 & 8.45 & 9.33 & 17.88 & 18.80 & 34.00 & 29.05 & 29.57 & 37.60 & 38.04 & 38.24 & 40.73 & 40.92 \\
\hline Laboratory: & $\begin{array}{c}\text { Shipboard } \\
\text { XRF }\end{array}$ & ICP-AES-UH & ICP-AES-UH & $\begin{array}{c}\text { Shipboard } \\
\text { XRF }\end{array}$ & ICP-AES-UH & ICP-AES-UH & ICP-AES-UH & $\begin{array}{l}\text { Shipboard } \\
\text { XRF }\end{array}$ & ICP-AES-UH & ICP-AES-UH & $\begin{array}{l}\text { Shipboard } \\
\text { XRF }\end{array}$ & $\begin{array}{l}\text { Shipboard } \\
\text { XRF }\end{array}$ & ICP-AES-UH \\
\hline Lithology: & Harzburgite & $\begin{array}{l}\text { Harzburgite/ } \\
\text { pyroxenite }\end{array}$ & $\begin{array}{l}\text { Harzburgite/ } \\
\text { pyroxenite }\end{array}$ & Harzburgite & Harzburgite & Harzburgite & Harzburgite & Harzburgite & Harzburgite & Harzburgite & Harzburgite & Harzburgite & Harzburgite \\
\hline \multicolumn{14}{|l|}{ Major elements (wt\%) } \\
\hline $\mathrm{SiO}_{2}$ & 44.75 & 45.55 & 45.74 & 44.09 & 45.01 & 46.54 & 43.97 & 45.09 & 43.93 & 43.83 & 43.51 & 44.64 & 44.65 \\
\hline $\mathrm{TiO}_{2}$ & 0.03 & 0.05 & 0.11 & 0.02 & 0.02 & 0.04 & 0.02 & 0.02 & 0.03 & 0.03 & 0.02 & 0.02 & 0.03 \\
\hline $\mathrm{Al}_{2} \mathrm{O}_{3}$ & 1.49 & 1.58 & 3.40 & 1.43 & 1.54 & 1.78 & 1.38 & 1.49 & 1.45 & 1.54 & 1.57 & 1.45 & 1.39 \\
\hline $\mathrm{Fe}_{2} \mathrm{O}_{3}$ & 9.30 & 10.34 & 12.05 & 9.21 & 9.01 & 8.47 & 9.13 & 9.04 & 8.84 & 9.08 & 9.21 & 9.02 & 9.38 \\
\hline $\mathrm{MnO}$ & 0.12 & 0.13 & 0.26 & 0.12 & 0.13 & 0.13 & 0.12 & 0.12 & 0.14 & 0.12 & 0.14 & 0.15 & 0.13 \\
\hline $\mathrm{MgO}$ & 43.56 & 40.49 & 34.17 & 44.17 & 42.73 & 40.54 & 43.49 & 42.74 & 44.19 & 44.48 & 43.99 & 43.60 & 43.85 \\
\hline $\mathrm{CaO}$ & 0.33 & 0.77 & 4.19 & 0.07 & 0.00 & 1.30 & 0.00 & 0.39 & 0.42 & 0.02 & 0.10 & $\begin{array}{r}43.00 \\
0.01\end{array}$ & 0.04 \\
\hline $\mathrm{Na}_{2} \mathrm{O}$ & 0.00 & 0.00 & 0.14 & 0.00 & 0.00 & 0.07 & 0.00 & 0.00 & 0.05 & 0.06 & 0.00 & 0.00 & 0.06 \\
\hline $\mathrm{K}_{2} \mathrm{O}$ & 0.01 & 0.00 & 0.00 & 0.00 & 0.00 & 0.03 & 0.00 & 0.00 & 0.03 & 0.02 & 0.00 & 0.00 & 0.02 \\
\hline $\mathrm{P}_{2} \mathrm{O}_{5}$ & 0.00 & 0.02 & 0.04 & 0.00 & 0.00 & 0.04 & 0.00 & 0.01 & 0.01 & 0.01 & 0.00 & 0.00 & 0.01 \\
\hline $\mathrm{Cr}_{2} \mathrm{O}_{3}$ & 0.42 & 0.35 & 0.38 & 0.43 & 0.50 & 0.42 & 0.43 & 0.39 & 0.39 & 0.43 & 0.43 & 0.42 & 0.42 \\
\hline $\mathrm{NiO}$ & 0.33 & 0.31 & 0.24 & 0.34 & 0.35 & 0.29 & 0.33 & 0.31 & 0.32 & 0.33 & 0.32 & 0.33 & 0.37 \\
\hline Total & 100.33 & 99.59 & 100.72 & 99.87 & 99.28 & 99.63 & 98.87 & 99.60 & 99.78 & 99.95 & 99.29 & 99.64 & 100.35 \\
\hline LOI & 11.67 & 12.21 & 7.13 & 12.06 & 12.05 & 8.21 & 12.21 & 11.01 & 12.47 & 12.29 & 12.35 & 12.07 & \\
\hline $\mathrm{CO}_{2}$ & 0.51 & & & 0.36 & & & & 0.41 & & & 0.33 & 0.3 & \\
\hline $\mathrm{H}_{2} \mathrm{O}$ & 10.9 & & & 12.5 & & & & 11.5 & & & 12.2 & 12.2 & \\
\hline $\mathrm{FeOT}$ & 8.37 & 9.30 & 10.84 & 8.29 & 8.11 & 7.62 & 8.22 & 8.13 & 7.96 & 8.17 & 8.29 & 8.12 & 8.44 \\
\hline $\mathrm{Mg \#}$ & 90.27 & 88.58 & 84.88 & 90.47 & 90.38 & 90.46 & 90.42 & 90.35 & 90.82 & 90.66 & 90.44 & 90.54 & 90.25 \\
\hline $\mathrm{Mg \# +2}$ & 91.61 & 90.12 & 86.85 & 91.79 & 91.70 & 91.77 & 91.73 & 91.68 & 92.09 & 91.94 & 91.75 & 91.84 & 91.59 \\
\hline FeOT/MgO & 0.19 & 0.23 & 0.32 & 0.19 & 0.19 & 0.19 & 0.19 & 0.19 & 0.18 & 0.18 & 0.19 & 0.19 & 0.19 \\
\hline \multicolumn{14}{|l|}{ Trace elements (ppm) } \\
\hline $\mathrm{V}$ & 53 & 68 & 62 & 50 & 62 & 72 & 63 & 55 & 55 & 63 & 54 & 57 & 91 \\
\hline $\mathrm{Ti}$ & 180 & 300 & 659 & 120 & 120 & 215 & 120 & 120 & 150 & 150 & 120 & 120 & 163 \\
\hline $\mathrm{Cr}$ & 2864 & 2369 & 2592 & 2910 & 3413 & 2850 & 2929 & 2658 & 2653 & 2962 & 2921 & 2873 & 2894 \\
\hline $\mathrm{Ni}$ & 2564 & 2461 & 1927 & 2641 & 2723 & 2292 & 2612 & 2469 & 2510 & 2621 & 2533 & 2600 & 2935 \\
\hline $\mathrm{Sr}$ & $\begin{array}{r}204 \\
8\end{array}$ & $\begin{array}{r}2401 \\
39\end{array}$ & $\begin{array}{r}1927 \\
12\end{array}$ & $\begin{array}{r}20+1 \\
9\end{array}$ & 8 & 10 & $\begin{array}{l}2012 \\
15\end{array}$ & $\begin{aligned} 2409 \\
8\end{aligned}$ & 73 & ${ }_{11}^{2021}$ & 2539 & 8000 & 11 \\
\hline $\mathrm{Zr}$ & 8 & 226 & 507 & 4 & $\begin{array}{l}8 \\
4\end{array}$ & 8 & 3 & 4 & 6 & 11 & 3 & 4 & 11 \\
\hline $\mathrm{Ba}$ & 0 & 16 & 15 & 0 & 15 & $\stackrel{8}{16}$ & 15 & 0 & 16 & 16 & 0 & 0 & 14 \\
\hline $\mathrm{Sc}$ & & 11.34 & 17.73 & & & & 23.71 & & 11.55 & & & & \\
\hline $\mathrm{Y}$ & & 9.72 & 48.99 & & & & 0.92 & & 0.25 & & & & \\
\hline \multicolumn{14}{|l|}{ Rare earths (ppm) } \\
\hline $\mathrm{La}$ & & 0.63 & 4.79 & & & & 0.64 & & 0.22 & & & & \\
\hline $\mathrm{Ce}$ & & 2.29 & 16.70 & & & & 1.39 & & 0.31 & & & & \\
\hline $\mathrm{Nd}$ & & 2.45 & 12.72 & & & & 0.19 & & & & & & \\
\hline $\mathrm{Sm}$ & & 0.96 & $\begin{array}{r}4.54 \\
4.54\end{array}$ & & & & 0.23 & & & & & & \\
\hline $\mathrm{Eu}$ & & 0.16 & $\begin{array}{l}4.34 \\
1.15\end{array}$ & & & & 0.25 & & 0.04 & & & & \\
\hline Gd & & $\begin{array}{l}.10 \\
1.53\end{array}$ & 6.65 & & & & 1.16 & & 0.38 & & & & \\
\hline Dy & & 1.48 & 7.79 & & & & 0.04 & & 0.02 & & & & \\
\hline Er & & 1.15 & 5.86 & & & & 0.18 & & 0.07 & & & & \\
\hline $\mathrm{Yb}$ & & 1.16 & 5.39 & & & & 0.21 & & 0.09 & & & & \\
\hline $\mathrm{Lu}$ & & & & & & & 0.02 & & & & & & \\
\hline
\end{tabular}

Notes: $\mathrm{MgH}+2=100 \times \mathrm{Mg} /\left(\mathrm{Mg}+\mathrm{Fe}^{2+}\right)$, where $\left.\mathrm{Fe}^{3+} /\left[\mathrm{Fe}^{2+}+\mathrm{Fe}^{3+}\right]\right)$ is equal to 0.10 . D. harzburgite = harzburgite depleted in pyroxene, E. harzburgite = harzburgite enriched in pyroxene. Other abbreviations symbols and abbreviations as in Table 1. 
Table 2 (continued).

\begin{tabular}{|c|c|c|c|c|c|c|c|c|c|c|c|c|c|}
\hline Hole: & 920D & 920D & 920D & 920D & 920D & 920D & 920D & 920D & 920D & 920D & 920D & 920D & 920D \\
\hline Core, section, interval $(\mathrm{cm})$ : & : $6 \mathrm{R}-1,82-88$ & $6 \mathrm{R}-2,45-51$ & $7 R-1,76-83$ & $7 R-2,22-29$ & $7 R-2,22-29$ & $8 \mathrm{R}-1,123-12$ & $8 R-2,82-90$ & $8 R-3,66-73$ & $8 R-3,80-85$ & $10 R-1,57-60$ & 10R-1, 101-107 & $10 \mathrm{R}-2,48-55$ & $10 R-3,15-20$ \\
\hline Depth (mbsf): & 47.32 & 48.27 & 56.96 & 57.89 & 57.89 & 67.13 & 68.16 & 69.46 & 69.60 & 78.67 & 79.11 & 79.97 & 81.11 \\
\hline Laboratory: & ICP-AES-UH & $\begin{array}{c}\text { Shipboard } \\
\text { XRF }\end{array}$ & ICP-AES-UH & $\begin{array}{l}\text { Shipboard } \\
\text { XRF }\end{array}$ & ICP-AES-UH & $\begin{array}{c}\text { Shipboard } \\
\text { XRF }\end{array}$ & ICP-AES-UH & $\begin{array}{l}\text { Shipboard } \\
\text { XRF }\end{array}$ & ICP-AES-UH & $\begin{array}{c}\text { Shipboard } \\
\text { XRF }\end{array}$ & ICP-AES-UH & ICP-AES-UH & ICP-AES-UH \\
\hline Lithology: & Harzburgite & Harzburgite & Harzburgite & Gabbro & $\begin{array}{l}\text { Amphibolite } \\
\text { microgabbro }\end{array}$ & Harzburgite & Harzburgite & Diabase & Diabase & Diabase & D. Harzburgite & $\begin{array}{l}\text { Pyroxenite/ } \\
\text { harzburgite }\end{array}$ & D. harzburgite \\
\hline \multicolumn{14}{|l|}{ Major elements (wt\%) } \\
\hline $\mathrm{SiO}_{2}$ & 44.94 & 44.81 & 45.77 & 45.10 & 44.99 & 43.71 & 44.92 & 48.10 & 48.94 & 50.21 & 46.02 & 44.39 & 44.81 \\
\hline $\mathrm{TiO} 2$ & 0.02 & 0.01 & 0.43 & 1.22 & 1.20 & 0.01 & 0.03 & 0.99 & 0.96 & 1.11 & 0.02 & 0.41 & 0.03 \\
\hline $\mathrm{Al}_{2} \mathrm{O}_{3}$ & 1.49 & 1.29 & 1.98 & 16.68 & 17.31 & 1.31 & 1.67 & 16.68 & 17.79 & 14.93 & 1.56 & 6.88 & 1.49 \\
\hline $\mathrm{Fe}_{2} \mathrm{O}_{3}$ & 9.07 & 9.29 & 12.92 & 12.97 & 12.75 & 9.60 & 9.37 & 8.86 & 8.78 & 9.55 & 9.14 & 12.72 & 8.75 \\
\hline $\mathrm{MnO}$ & 0.12 & 0.13 & 0.18 & 0.23 & 0.23 & 0.14 & 0.13 & 0.25 & 0.23 & 0.28 & 0.11 & 0.19 & 0.11 \\
\hline $\mathrm{MgO}$ & 42.46 & 43.66 & 35.79 & 12.07 & 11.62 & 43.61 & 42.59 & 14.00 & 12.60 & 15.08 & 42.44 & 30.19 & 43.94 \\
\hline $\mathrm{CaO}$ & 0.15 & 0.02 & 2.26 & 8.61 & 8.59 & 0.08 & 0.38 & 7.73 & 8.42 & 6.21 & 0.00 & 5.07 & 0.18 \\
\hline $\mathrm{Na}_{2} \mathrm{O}$ & 0.00 & 0.00 & 0.17 & 2.33 & 2.77 & 0.00 & 0.00 & 2.70 & 2.91 & 3.36 & 0.00 & 0.15 & 0.03 \\
\hline $\mathrm{K}_{2} \mathrm{O}$ & 0.00 & 0.00 & 0.00 & 0.05 & 0.03 & 0.00 & 0.00 & 0.02 & 0.05 & 0.02 & 0.00 & 0.00 & 0.01 \\
\hline $\mathrm{P}_{2} \mathrm{O}_{5}$ & 0.01 & 0.00 & 0.04 & 0.04 & 0.06 & 0.00 & 0.01 & 0.04 & 0.08 & 0.04 & 0.01 & 0.33 & 0.01 \\
\hline $\mathrm{Cr}_{2} \mathrm{O}_{3}$ & 0.42 & 0.43 & 0.30 & 0.05 & 0.06 & 0.42 & 0.48 & 0.06 & 0.07 & 0.07 & 0.44 & 0.39 & 0.48 \\
\hline $\mathrm{NiO}$ & 0.32 & 0.33 & 0.25 & 0.04 & 0.04 & 0.34 & 0.33 & 0.04 & 0.03 & 0.04 & 0.29 & 0.17 & 0.34 \\
\hline Total & 99.00 & 99.97 & 100.09 & 99.39 & 99.65 & 99.21 & 99.91 & 99.47 & 100.86 & 100.90 & 100.03 & 100.89 & 100.18 \\
\hline $\begin{array}{l}\mathrm{LOI} \\
\mathrm{CO}_{2}\end{array}$ & 11.75 & $\begin{array}{r}12.50 \\
0.47\end{array}$ & 5.04 & $\begin{array}{l}2.56 \\
0.05\end{array}$ & 2.68 & $\begin{array}{r}12.09 \\
0.41\end{array}$ & 11.07 & $\begin{array}{l}4.49 \\
0.04\end{array}$ & 4,01 & $\begin{array}{l}4.58 \\
0.06\end{array}$ & 12.20 & 8.43 & 11.63 \\
\hline $\mathrm{H}_{2} \mathrm{O}$ & & 11 & & 4.13 & & 13 & & 5.75 & & 5.71 & & & \\
\hline FeOT & 8.16 & 8.36 & 11.63 & 11.67 & 11.47 & 8.64 & 8.43 & 7.97 & 7.90 & 8.59 & 8.22 & 11.45 & 7.88 \\
\hline Mg\# & 90.26 & 90.30 & 84.58 & 64.83 & 64.35 & 90.00 & 90.00 & 75.78 & 73.97 & 75.77 & 90.19 & 82.46 & 90.86 \\
\hline $\mathrm{Mg \# +2}$ & 91.60 & 91.63 & 86.58 & 68.44 & 67.98 & 91.37 & 91.37 & 78.64 & 76.97 & 78.63 & 91.54 & 84.69 & 92.12 \\
\hline $\mathrm{FeOT} / \mathrm{MgO}$ & 0.19 & 0.19 & 0.32 & 0.97 & 0.99 & 0.20 & 0.20 & 0.57 & 0.63 & 0.57 & 0.19 & 0.38 & 0.18 \\
\hline \multicolumn{14}{|l|}{ Trace elements (ppm): } \\
\hline $\mathrm{V}$ & 48 & 49 & 95 & 223 & 199 & 50 & 73 & 177 & 152 & 178 & 57 & 71 & 66 \\
\hline $\mathrm{Ti}$ & 120 & 60 & 2578 & 7314 & 7194 & 60 & 180 & 5935 & 5752 & 6654 & 120 & 2458 & 193 \\
\hline $\mathrm{Cr}$ & 2848 & 2909 & 2034 & 343 & 413 & 2844 & 3295 & 427 & 473 & 489 & 3011 & 2674 & 3269 \\
\hline $\mathrm{Ni}$ & 2517 & 2612 & 1965 & 305 & 344 & 2663 & 2604 & 277 & 251 & 281 & 2322 & 1330 & 2673 \\
\hline $\mathrm{Sr}$ & 13 & 8 & 10 & 110 & 129 & 8 & 15 & 287 & 334 & 300 & 14 & 13 & 10 \\
\hline $\mathrm{Zr}$ & 6 & 4 & 31 & 67 & 78 & 4 & 8 & 69 & 72 & 75 & 5 & 253 & 7 \\
\hline $\mathrm{Ba}$ & 18 & 0 & 14 & 7 & 20 & 0 & 16 & 17 & 20 & 23 & 10 & 13 & 17 \\
\hline $\mathrm{Sc}$ & & & & 42.96 & 44.50 & & 10.81 & 30.99 & 31.58 & 33.83 & & 19.50 & \\
\hline $\mathrm{Y}$ & & & & 37.40 & 37.39 & & 0.32 & 21.33 & 22.70 & 23.97 & & 61.79 & \\
\hline \multicolumn{14}{|l|}{ Rare earths (ppm): } \\
\hline $\mathrm{La}$ & & & & 2.26 & 2.41 & & 0.66 & 5.08 & 2.53 & 2.70 & & 8.45 & \\
\hline $\mathrm{Ce}$ & & & & 8.64 & 8.75 & & 1.69 & 7.82 & 8.79 & 8.52 & & 27.15 & \\
\hline $\mathrm{Nd}$ & & & & 6.94 & 7.71 & & & 6.36 & 6.59 & 6.66 & & 18.52 & \\
\hline $\mathrm{Sm}$ & & & & 2.53 & 2.77 & & & 2.04 & 2.16 & 2.22 & & 6.48 & \\
\hline $\mathrm{Eu}$ & & & & 1.15 & 1.22 & & 0.01 & 0.74 & 0.84 & 0.93 & & 1.28 & \\
\hline Gd & & & & 3.80 & 4.09 & & 0.51 & 2.68 & 2.92 & 3.04 & & 9.33 & \\
\hline Dy & & & & 5.52 & 5.52 & & & 3.33 & 3.56 & 3.70 & & 9.76 & \\
\hline Er & & & & 3.89 & 4.01 & & 0.12 & 2.28 & 2.32 & 2.40 & & 6.35 & \\
\hline $\mathrm{Yb}$ & & & & 3.87 & 3.86 & & 0.09 & 2.05 & 2.21 & 2.35 & & 5.63 & \\
\hline Lu & & & & & 0.64 & & & & & 0.39 & & & \\
\hline
\end{tabular}


Table 2 (continued).

\begin{tabular}{|c|c|c|c|c|c|c|c|c|c|c|c|c|c|}
\hline Hole: & $920 \mathrm{D}$ & $920 \mathrm{D}$ & $920 \mathrm{D}$ & $920 \mathrm{D}$ & 920D & 920D & 920D & 920D & 920D & 920D & 920D & 920D & 920D \\
\hline Core, section, interval $(\mathrm{cm})$ : & $10 \mathrm{R}-3,74-80$ & $11 \mathrm{R}-2,63-69$ & $11 R-3,22-28$ & $11 R-4,16-22$ & $12 \mathrm{R}-2,119-12$ & $12 \mathrm{R}-3,50-57$ & $12 \mathrm{R}-3,96-100$ & $12 \mathrm{R}-5,23-29$ & $13 \mathrm{R}-1,62-70 \mathrm{~A}$ & $13 R-1,62-70 B$ & $13 \mathrm{R}-2,51-57$ & $14 \mathrm{R}-3,37-43$ & $14 \mathrm{R}-3,82-88$ \\
\hline Depth (mbsf): & 81.70 & 87.35 & 88.14 & 89.45 & 97.64 & 98.23 & 98.69 & 100.76 & 105.22 & 105.22 & 106.00 & 117.40 & 117.85 \\
\hline Laboratory: & $\begin{array}{l}\text { Shipboard } \\
\text { XRF }\end{array}$ & $\begin{array}{c}\text { Shipboard } \\
\text { XRF }\end{array}$ & $\begin{array}{c}\text { Shipboard } \\
\text { XRF }\end{array}$ & ICP-AES-UH & $\begin{array}{c}\text { Shipboard } \\
\text { XRF }\end{array}$ & ICP-AES-UH & ICP-AES-UH & $\begin{array}{c}\text { Shipboard } \\
\text { XRF }\end{array}$ & ICP-AES-UH & ICP-AES-UH & ICP-AES-UH & ICP-AES-UH & $\begin{array}{l}\text { Shipboard } \\
\text { XRF }\end{array}$ \\
\hline Lithology & Harzburgite & Harzburgite & Harzburgite & E. Harzburgite & Harzburgite & Harzburgite & $\begin{array}{l}\text { Anorthositic } \\
\text { dike }\end{array}$ & 'Harzburgite & Harzburgite & $\begin{array}{l}\text { Pyroxenite/ } \\
\text { wehrlite }\end{array}$ & Harzburgite & Harzburgite & Harzburgite \\
\hline \multicolumn{14}{|l|}{ Major elements (wt\%) } \\
\hline $\mathrm{STO}_{2}$ & 44.11 & 43.70 & 44.48 & 44.97 & 43.68 & 47.22 & 55.02 & 43.80 & 44.63 & 47.12 & 44.31 & 44.25 & 43.26 \\
\hline $\mathrm{TiO}_{2}$ & 0.01 & 0.02 & 0.03 & 0.03 & 0.01 & 0.04 & 0.44 & 0.02 & 0.02 & 0.47 & 0.02 & 0.02 & 0.01 \\
\hline $\mathrm{Al}_{2} \mathrm{O}_{3}$ & 1.34 & 1.49 & 1.48 & 1.83 & 1.28 & 1.69 & 20.30 & 1.35 & 1.43 & 2.32 & 1.21 & 1.38 & 1.41 \\
\hline $\mathrm{Fe}_{2} \mathrm{O}_{3}$ & 9.52 & 9.92 & 8.84 & 8.98 & 9.31 & 8.55 & 5.42 & 9.12 & 10.21 & 14.52 & 9.37 & 9.35 & 9.08 \\
\hline & 0.14 & 0.15 & 0.14 & 0.13 & 0.12 & 0.14 & 0.10 & 0.15 & 0.12 & 0.27 & 0.13 & 0.13 & 0.14 \\
\hline $\mathrm{MgO}$ & 44.09 & 43.54 & 43.87 & 42.82 & 43.83 & 41.47 & 3.61 & 44.29 & 43.39 & 28.82 & 43.79 & 43.48 & 43.52 \\
\hline $\mathrm{CaO}$ & 0.29 & 0.03 & 0.29 & 1.38 & 0.23 & 1.06 & 8.97 & 0.26 & 0.51 & 6.20 & 0.42 & 1.30 & 1.55 \\
\hline $\mathrm{Na}_{2} \mathrm{O}$ & 0.00 & 0.00 & 0.00 & 0.03 & 0.00 & 0.00 & 6.54 & 0.00 & 0.05 & 0.12 & 0.00 & 0.00 & 0.00 \\
\hline $\mathrm{K}_{2} \mathrm{O}$ & 0.00 & 0.00 & 0.00 & 0.01 & 0.00 & 0.00 & 0.13 & 0.00 & 0.02 & 0.00 & 0.00 & 0.00 & 0.00 \\
\hline $\mathrm{P}_{2} \mathrm{O}_{5}$ & 0.00 & 0.00 & 0.00 & 0.01 & 0.00 & 0.01 & 0.01 & 0.00 & 0.01 & 0.01 & 0.00 & 0.00 & 0.00 \\
\hline $\mathrm{Cr}_{2} \mathrm{O}_{3}$ & 0.38 & 0.50 & 0.48 & 0.49 & 0.38 & 0.43 & 0.02 & 0.42 & 0.36 & 0.25 & 0.30 & 0.41 & 0.43 \\
\hline $\mathrm{NiO}^{3}$ & 0.35 & 0.33 & 0.33 & 0.22 & 0.34 & 0.31 & 0.01 & 0.33 & 0.31 & 0.15 & 0.32 & 0.28 & 0.32 \\
\hline Total & 100.23 & 99.68 & 99.94 & 100.90 & 99.18 & 100.92 & 100.57 & 99.74 & 101.07 & 100.25 & 99.87 & 100.60 & 99.73 \\
\hline & 11.90 & 12.26 & 12.42 & 8.89 & 12.44 & 9.11 & 0.90 & 12.76 & 12.53 & 2.78 & 11.56 & 8.86 & 10.36 \\
\hline $\mathrm{CO}_{2}$ & 0.76 & & 0.73 & & & 0.74 & & 0.85 & & & & & 0.73 \\
\hline $\mathrm{H}_{2} \mathrm{O}$ & 10.8 & 12.8 & 12.1 & & & 12.5 & & & & & & & \\
\hline FeOT & 8.57 & 8.93 & 7.95 & 8.08 & 8.38 & 7.69 & 4.88 & 8.21 & 9.18 & 13.07 & 8.43 & 8.41 & 8.17 \\
\hline Mg\# & 90.17 & 89.68 & 90.76 & 90.43 & 90.31 & 90.57 & 56.88 & 90.58 & 89.38 & 79.72 & 90.25 & 90.21 & 90.47 \\
\hline $\mathrm{Mg \# +2}^{2}$ & 91.52 & 91.09 & 92.04 & 91.75 & 91.65 & 91.87 & 60.81 & 91.88 & 90.83 & 82.22 & 91.59 & 91.55 & 91.78 \\
\hline $\mathrm{FeOT} / \mathrm{MgO}$ & 0.19 & 0.21 & 0.18 & 0.19 & 0.19 & 0.19 & 1.35 & 0.19 & 0.21 & 0.45 & 0.19 & 0.19 & 0.19 \\
\hline \multicolumn{14}{|l|}{ Trace elements (ppm) } \\
\hline $\mathrm{V}$ & 50 & 54 & 59 & 53 & 49 & 80 & 93 & 56 & 49 & 197 & 48 & 41 & 51 \\
\hline $\mathrm{Ti}$ & 60 & 120 & 180 & 159 & 60 & 240 & 2638 & 120 & 140 & 2818 & 120 & 120 & 60 \\
\hline $\mathrm{Cr}$ & 2609 & 3395 & 3297 & 3354 & 2580 & 2949 & 134 & 2907 & 2485 & 1727 & 2075 & 2808 & 2974 \\
\hline $\mathrm{Ni}$ & 2733 & 2622 & 2579 & 1734 & 2662 & 2435 & 56 & 2566 & 2447 & 1151 & 2532 & 2222 & 2529 \\
\hline $\mathrm{Sr}$ & 8 & 8 & 8 & 3 & 16 & 9 & 327 & 8 & 42 & 8 & 4 & 4 & 7 \\
\hline $\mathrm{Zr}$ & 4 & 5 & 5 & 7 & 4 & 10 & 24 & 4 & 4 & 17 & 4 & 5 & 5 \\
\hline $\mathrm{Ba}$ & 0 & 0 & 0 & 14 & 0 & 14 & 34 & 0 & 18 & 11 & 9 & 12 & 0 \\
\hline $\begin{array}{l}\mathrm{Sc} \\
\mathrm{Y}\end{array}$ & & & & & & & 23.30 & & & 46.16 & & & \\
\hline $\mathrm{Y}$ & & & & & & & 18.95 & & & 12.01 & & & \\
\hline \multicolumn{14}{|l|}{ Rare earths (ppm) } \\
\hline $\mathrm{La}$ & & & & & & & 1.98 & & & 0.24 & & & \\
\hline $\mathrm{Ce}$ & & & & & & & 6.25 & & & 1.24 & & & \\
\hline $\mathrm{Nd}$ & & & & & & & 4.07 & & & 1.58 & & & \\
\hline $\mathrm{Sm}$ & & & & & & & 1.49 & & & 0.74 & & & \\
\hline $\mathrm{Eu}$ & & & & & & & 2.90 & & & 0.21 & & & \\
\hline $\mathrm{Gd}$ & & & & & & & 2.24 & & & 1.42 & & & \\
\hline Dy & & & & & & & 3.05 & & & 1.83 & & & \\
\hline Er & & & & & & & 2.11 & & & 1.32 & & & \\
\hline $\mathrm{Yb}$ & & & & & & & 2.01 & & & 1.29 & & & \\
\hline Lu & & & & & & & & & & & & & \\
\hline
\end{tabular}


Table 2 (continued).

\begin{tabular}{|c|c|c|c|c|c|c|c|c|c|c|c|c|c|c|}
\hline Hole: & 920D & $920 \mathrm{D}$ & 920D & 920D & 920D & 920D & 920D & 920D & 920D & 920D & 920D & 920D & 920D & 920D \\
\hline Core, section, interval $(\mathrm{cm})$ & $14 R-4,103-110$ & $15 \mathrm{R}-3,40-45$ & $15 R-3,48-54$ & $16 \mathrm{R}-1,98-10$ & $16 R-7,54-60$ & $17 R-3,37-43$ & $18 \mathrm{R}-2,92-98$ & $18 \mathrm{R}-3,29-35$ & $18 \mathrm{R}-3,75-81$ & $18 R-3,93-98$ & $19 \mathrm{R}-2,13-19$ & $20 \mathrm{R}-1,103-1$ & 1) $20 \mathrm{R}-2,24-30$ & $20 R-4,38-44$ \\
\hline Depth (mbsf): & 119.52 & 126.91 & 126.99 & 134.38 & 141.53 & 145.74 & 155.02 & 155.64 & 156.10 & 156.26 & 163.80 & 172.93 & 173.30 & 175.92 \\
\hline Laboratory: & ICP-AES-UH & $\begin{array}{c}\text { Shipboard } \\
\text { XRF }\end{array}$ & $\begin{array}{c}\text { Shipboard } \\
\text { XRF }\end{array}$ & $\begin{array}{c}\text { Shipboard } \\
\text { XRF }\end{array}$ & $\begin{array}{c}\text { Shipboard } \\
\text { XRF }\end{array}$ & $\begin{array}{c}\text { Shipboard } \\
\text { XRF }\end{array}$ & ICP-AES-UH & ICP-AES-UH & $\begin{array}{c}\text { Shipboard } \\
\text { XRF }\end{array}$ & ICP-AES-UH & $\begin{array}{c}\text { Shipboard } \\
\text { XRF }\end{array}$ & $\begin{array}{c}\text { Shipboard } \\
\text { XRF }\end{array}$ & $\begin{array}{c}\text { Shipboard } \\
\text { XRF }\end{array}$ & $\begin{array}{l}\text { Shipboard } \\
\text { XRF }\end{array}$ \\
\hline Lithology: & Harzburgite & Harzburgite & Harzburgite & Harzburgite & Harzburgite & Harzburgite & $\begin{array}{c}\text { Harzburgite/ } \\
\text { pyroxenite }\end{array}$ & Harzburgite & Harzburgite & $\begin{array}{l}\text { Harzburgite/ } \\
\text { pyroxenite }\end{array}$ & Harzburgite & Harzburgite & Harzburgite & Harzburgite \\
\hline \multicolumn{15}{|l|}{ Major elements (wt\%) } \\
\hline $\mathrm{SiO}_{2}$ & 43.28 & 42.85 & 43.00 & 43.79 & 44.02 & 43.98 & 46.64 & 44.10 & 43.63 & 44.38 & 44.27 & 43.79 & 44.29 & 43.90 \\
\hline $\mathrm{TiO}_{2}^{2}$ & 0.02 & 0.01 & 0.01 & 0.02 & 0.01 & 0.02 & 0.06 & 0.07 & 0.01 & 0.07 & 0.02 & 0.01 & 0.01 & 0.01 \\
\hline $\mathrm{Al}_{2} \mathrm{O}_{3}$ & 1.55 & 1.49 & 1.34 & 1.50 & 1.30 & 1.51 & 1.50 & 1.44 & 1.39 & 1.43 & 1.34 & 1.36 & 1.64 & 1.48 \\
\hline $\mathrm{Fe}_{2} \mathrm{O}_{3}$ & 8.53 & 10.46 & 9.36 & 8.99 & 9.52 & 9.25 & 11.32 & 11.20 & 10.90 & 11.60 & 9.71 & 9.34 & 9.05 & 9.11 \\
\hline $\mathrm{MnO}$ & 0.13 & 0.16 & 0.14 & 0.15 & 0.13 & 0.14 & 0.16 & 0.16 & 0.15 & 0.16 & 0.14 & 0.14 & 0.15 & 0.14 \\
\hline $\mathrm{MgO}$ & 43.19 & 44.05 & 44.23 & 43.97 & 44.39 & 43.90 & 38.02 & 40.55 & 42.74 & 40.63 & 43.33 & 44.14 & 43.49 & 43.85 \\
\hline $\mathrm{CaO}$ & 1.05 & 0.58 & 0.99 & 1.15 & 0.12 & 0.39 & 1.05 & 0.32 & 0.14 & 0.79 & 0.25 & 0.23 & 0.26 & 0.39 \\
\hline $\mathrm{Na}_{2} \mathrm{O}$ & 0.03 & 0.00 & 0.00 & 0.00 & 0.00 & 0.00 & 0.11 & 0.00 & 0.00 & 0.06 & 0.00 & 0.00 & 0.00 & 0.00 \\
\hline $\mathrm{K}_{2} \mathrm{O}$ & 0.02 & 0.00 & 0.00 & 0.00 & 0.00 & 0.00 & 0.03 & 0.00 & 0.00 & 0.02 & 0.00 & 0.00 & 0.00 & 0.00 \\
\hline $\mathrm{P}_{2} \mathrm{O}_{5}$ & 0.01 & 0.00 & 0.00 & 0.00 & 0.00 & 0.00 & 0.01 & 0.00 & 0.00 & 0.01 & 0.00 & 0.00 & 0.00 & 0.00 \\
\hline $\mathrm{Cr}_{2} \mathrm{O}_{3}$ & 0.46 & 0.53 & 0.42 & 0.41 & 0.39 & 0.50 & 0.41 & 0.36 & 0.45 & 0.37 & 0.42 & 0.42 & 0.48 & 0.47 \\
\hline 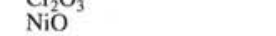 & 0.28 & 0.33 & 0.34 & 0.32 & 0.35 & 0.34 & 0.29 & 0.29 & 0.32 & 0.29 & 0.33 & 0.34 & 0.31 & 0.33 \\
\hline Total & 98.55 & 100.46 & 99.84 & 100.31 & 100.23 & 100.02 & 99.58 & 98.49 & 99.73 & 99.82 & 99.82 & 99.77 & 99.68 & 99.67 \\
\hline LOI & 10.92 & 12.34 & 11.91 & 11.23 & 12.16 & 12.17 & 9.14 & 11.06 & 12.10 & 10.41 & 11.86 & 12.30 & 11.83 & 12.02 \\
\hline $\mathrm{CO}_{2}$ & & 0.54 & 0.82 & 0.74 & 0.4 & 0.51 & & & 0.38 & & 0.48 & 0.63 & 0.63 & 0.56 \\
\hline $\mathrm{H}_{2} \mathrm{O}$ & & 12.1 & 10.1 & 10.7 & 12.5 & 11.8 & & & 12 & & 11.2 & 12.4 & 12.1 & 11.5 \\
\hline FéOT & 7.67 & 9.41 & 8.42 & 8.09 & 8.57 & 8.32 & 10.18 & 10.08 & 9.81 & 10.44 & 8.74 & 8.40 & 8.14 & 8.20 \\
\hline Mg\# & 90.94 & 89.29 & 90.35 & 90.64 & 90.23 & 90.38 & 86.93 & 87.76 & 88.59 & 87.40 & 89.83 & 90.35 & 90.49 & 90.51 \\
\hline $\mathrm{Mg \#}^{+2}$ & 92.19 & 90.75 & 91.67 & 91.93 & 91.57 & 91.71 & 88.67 & 89.40 & 90.13 & 89.08 & 91.23 & 91.67 & 91.80 & 91.81 \\
\hline $\mathrm{FeOT} / \mathrm{MgO}$ & 0.18 & 0.21 & 0.19 & 0.18 & 0.19 & 0.19 & 0.27 & 0.25 & 0.23 & 0.26 & 0.20 & 0.19 & 0.19 & 0.19 \\
\hline \multicolumn{15}{|l|}{ Trace elements (ppm) } \\
\hline $\mathrm{V}$ & 53 & 51 & 50 & 51 & 48 & 55 & 60 & 72 & 55 & 82 & 53 & 51 & 63 & 56 \\
\hline $\mathrm{Ti}$ & 140 & 60 & 60 & 120 & 60 & 120 & 333 & 420 & 60 & 417 & 120 & 60 & 60 & 60 \\
\hline $\mathrm{Cr}$ & 3154 & 3629 & 2896 & 2821 & 2683 & 3409 & 2823 & 2477 & 3071 & 2519 & 2898 & 2900 & 3264 & 3191 \\
\hline $\mathrm{Ni}$ & 2206 & 2560 & 2691 & 2541 & 2720 & 2642 & 2290 & 2292 & 2501 & 2294 & 2637 & 2648 & 2441 & 2582 \\
\hline $\mathrm{Sr}$ & 5 & 7 & 7 & 8 & 7 & 8 & 8 & 6 & 7 & 7 & 7 & 8 & 8 & 8 \\
\hline $\mathrm{Zr}$ & 4 & 4 & 4 & 4 & 4 & 4 & 7 & 6 & 5 & 11 & 5 & 4 & 5 & 4 \\
\hline $\mathrm{Ba}$ & 10 & 0 & 0 & 0 & 0 & 0 & 14 & 11 & 0 & i1 & 0 & 0 & 0 & 0 \\
\hline $\mathrm{Sc}$ & 25.72 & & & & & & & & & & & & & \\
\hline $\mathrm{Y}$ & 0.91 & & & & & & & & & & & & & \\
\hline \multicolumn{15}{|l|}{ Rare earths (ppm) } \\
\hline $\mathrm{La}$ & 0.67 & & & & & & & - & & & & & & \\
\hline $\mathrm{Ce}$ & 1.39 & & & & & & & & & & & & & \\
\hline $\begin{array}{l}\mathrm{Nd} \\
\mathrm{Sm}\end{array}$ & $\begin{array}{l}0.14 \\
0.20\end{array}$ & & & & & & & & & & & & & \\
\hline $\mathrm{Eu}$ & 0.22 & & & & & & & & & & & & & \\
\hline Gd & 1.12 & & & & & & & & & & & & & \\
\hline Dy & 0.03 & & & & & & & & & & & & & \\
\hline $\begin{array}{l}\mathrm{Er} \\
\mathrm{Yb}\end{array}$ & $\begin{array}{l}0.19 \\
0.22\end{array}$ & & & & & & & & & & & & & \\
\hline $\mathrm{Lu}$ & 0.02 & & & & & & & & & & & & & \\
\hline
\end{tabular}


Table 2 (continued).

\begin{tabular}{|c|c|c|c|c|c|c|c|c|c|c|c|c|}
\hline Hole: & $920 \mathrm{D}$ & 920D & 920D & 920D & 920D & 920D & 920D & 920D & 920D & 920D & 920D & 920D \\
\hline Core, section, interval $(\mathrm{cm})$ : & $21 R-2,40-46$ & $21 \mathrm{R}-3,57-66 \mathrm{~A}$ & 21R-3, 57-66B & $22 \mathrm{R}-2,36-43$ & $22 \mathrm{R}-2,86-92$ & $22 \mathrm{R}-3,6-14$ & $22 \mathrm{R}-4,33-38$ & $22 \mathrm{R}-5,12-19$ & $22 \mathrm{R}-5,104-1$ & $22 \mathrm{R}-5,129-135$ & $22 \mathrm{R}-6,0-8$ & $22 \mathrm{R}-7,16-22$ \\
\hline Depth (mbsf): & 183.15 & 184.67 & 184.67 & 192.16 & 192.66 & 192.79 & 193.76 & 194.75 & 195.67 & 195.92 & 196.04 & 197.49 \\
\hline Laboratory: & $\begin{array}{l}\text { Shipboard } \\
\text { XRF }\end{array}$ & ICP-AES-UH & ICP-AES-UH & ICP-AES-UH & $\begin{array}{l}\text { Shipboard } \\
\text { XRF }\end{array}$ & ICP-AES-UH & ICP-AES-UH & ICP-AES-UH & $\begin{array}{l}\text { Shipboard } \\
\text { XRF }\end{array}$ & ICP-AES-UH & ICP-AES-UH & $\begin{array}{c}\text { Shipboard } \\
\text { XRF }\end{array}$ \\
\hline Lithology & Harzburgite & Harzburgite & $\begin{array}{l}\text { Pyroxenitel } \\
\text { harzburgite }\end{array}$ & Harzburgite & Harzburgite & Harzburgite & Harzburgite & Harzburgite & Harzburgite & $\begin{array}{l}\text { Harzburgite/ } \\
\text { pyroxenite }\end{array}$ & Harzburgite & Harzburgite \\
\hline \multicolumn{13}{|l|}{ Major elements (wt\%) } \\
\hline $\mathrm{SiO}_{2}$ & 43.55 & 43.32 & 47.14 & 44.05 & 43.58 & 44.39 & 44.32 & 43.64 & 44.72 & 45.44 & 43.37 & 43.94 \\
\hline $\mathrm{TiO}_{2}^{-}$ & 0.01 & 0.02 & 1.09 & 0.03 & 0.01 & 0.03 & 0.03 & 0.02 & 0.01 & 0.68 & 0.02 & 0.01 \\
\hline $\mathrm{Al}_{2} \mathrm{O}_{3}$ & 1.50 & 1.25 & 3.27 & 1.52 & 1.45 & 1.36 & 1.75 & 1.38 & 1.61 & 6.96 & 1.18 & 1.49 \\
\hline $\mathrm{Fe}_{2} \mathrm{O}_{3}$ & 9.09 & 10.92 & 14.57 & 8.78 & 8.83 & 8.93 & 8.58 & 8.85 & 8.77 & 14.57 & 9.05 & 8.88 \\
\hline $\mathrm{MnO}$ & 0.14 & 0.14 & 0.28 & 0.12 & 0.13 & 0.13 & 0.13 & 0.12 & 0.14 & 0.25 & 0.10 & 0.14 \\
\hline $\mathrm{MgO}$ & 43.95 & 42.61 & 22.07 & 41.83 & 42.93 & 42.77 & 42.90 & 43.55 & 43.22 & 17.41 & 44.19 & 43.22 \\
\hline $\mathrm{CaO}$ & 0.73 & 0.17 & 11.07 & 1.42 & 1.41 & 1.25 & 1.33 & 0.32 & 0.57 & 14.55 & 0.10 & 1.39 \\
\hline $\mathrm{Na}_{2} \mathrm{O}$ & 0.00 & 0.03 & 0.36 & 0.00 & 0.00 & 0.00 & 0.03 & 0.04 & 0.00 & 0.30 & 0.05 & 0.00 \\
\hline $\mathrm{K}_{2} \mathrm{O}$ & 0.00 & 0.02 & 0.00 & 0.00 & 0.00 & 0.00 & 0.02 & 0.03 & 0.00 & 0.02 & 0.02 & 0.00 \\
\hline 等2 & 0.00 & 0.00 & 0.01 & 0.00 & 0.00 & 0.00 & 0.01 & 0.01 & 0.00 & 0.02 & 0.01 & 0.00 \\
\hline $\mathrm{Cr}_{2} \mathrm{O}_{3}$ & 0.46 & 0.41 & 0.26 & 0.44 & 0.39 & 0.39 & 0.50 & 0.36 & 0.45 & 0.07 & 0.37 & 0.39 \\
\hline $\mathrm{NiO}_{2}$ & 0.33 & 0.32 & $\begin{array}{l}0.20 \\
0.09\end{array}$ & 0.31 & 0.32 & 0.31 & 0.28 & 0.31 & 0.30 & 0.07 & 0.35 & 0.32 \\
\hline Total & 99.77 & 99.21 & 100.21 & 98.50 & 99.05 & 99.57 & 99.87 & 98.64 & 99.79 & 100.33 & 98.80 & 99.78 \\
\hline LOI & 12.21 & 11.88 & 2.70 & 10.15 & 9.62 & 8.97 & 9.91 & 12.34 & 11.99 & 4.49 & 13.66 & 10.97 \\
\hline $\mathrm{CO}_{2}$ & 0.46 & & & & 0.45 & & & & 0.55 & & & 0.71 \\
\hline $\begin{array}{l}\mathrm{H}_{2} \mathrm{O} \\
\mathrm{FeOT}\end{array}$ & & 9.82 & & & & 8.04 & & & & & & \\
\hline $\mathrm{FeOT}$ & 8.18 & 9.82 & 13.11 & 7.90 & 7.95 & 8.04 & 7.72 & 7.96 & 7.89 & 13.11 & 8.14 & 7.99 \\
\hline Mg\# & 90.54 & 88.54 & 75.00 & 90.42 & 90.59 & 90.46 & 90.83 & 90.69 & 90.71 & 70.30 & 90.63 & 90.60 \\
\hline Mg\#+2 & 91.85 & 90.09 & 77.92 & 91.74 & 91.89 & 91.78 & 92.09 & 91.98 & 91.99 & 73.58 & 91.92 & 91.90 \\
\hline $\mathrm{FeOT} / \mathrm{MgO}$ & 0.19 & 0.23 & 0.59 & 0.19 & 0.19 & 0.19 & 0.18 & 0.18 & 0.18 & 0.75 & 0.18 & 0.18 \\
\hline \multicolumn{13}{|l|}{ Trace elements (ppm) } \\
\hline $\mathrm{V}$ & 54 & 79 & 417 & 69 & 49 & 42 & 53 & 51 & 57 & 244 & 60 & 52 \\
\hline $\mathrm{Ti}$ & 60 & 128 & 6535 & 180 & 60 & 180 & 164 & 145 & 60 & 4056 & 146 & 60 \\
\hline $\mathrm{Cr}$ & 3177 & 2793 & 1751 & 3018 & 2664 & 2688 & 3449 & 2480 & 3051 & 510 & 2559 & 2640 \\
\hline $\mathrm{Ni}$ & 2628 & 2543 & 716 & 2468 & 2514 & 2480 & 2167 & 2433 & 2396 & 549 & 2745 & 2522 \\
\hline $\mathrm{Sr}$ & 8 & 11 & 15 & 5 & 8 & 10 & 8 & 2 & 8 & 23 & 9 & 8 \\
\hline $\mathrm{Zr}$ & 4 & 10 & 50 & 4 & 4 & 8 & 7 & 2 & $\frac{8}{5}$ & 30 & 6 & 8 \\
\hline $\mathrm{Ba}$ & 0 & 15 & 14 & 12 & 0 & 15 & 13 & $\frac{2}{7}$ & 0 & 19 & 19 & $\int_{0}^{0}$ \\
\hline $\mathrm{Sc}$ & & & $\begin{array}{l}14.09 \\
69.09\end{array}$ & & & & & & & & & \\
\hline $\mathrm{Y}$ & & & 30.83 & & & & & & & & & \\
\hline Rare earths (ppm) & & & & & & & & & & & & \\
\hline $\mathrm{La}$ & & & 0.90 & & & & & & & & & \\
\hline $\mathrm{Ce}$ & & & 4.12 & & & & & & & & & \\
\hline$\underset{N}{\mathrm{Nd}}$ & & & 5.33 & & & & & & & & & \\
\hline $\mathrm{Sm}$ & & & 2.18 & & & & & & & & & \\
\hline Eu & & & 0.91 & & & & & & & & & \\
\hline Gd & & & 3.67 & & & & & & & & & \\
\hline Dy & & & 4.81 & & & & & & & & & \\
\hline Er & & & 3.28 & & & & & & & & & \\
\hline $\begin{array}{l}\mathrm{Yb} \\
\mathrm{Lu}\end{array}$ & & & 2.99 & & & & & & & & & \\
\hline
\end{tabular}


Table 3. Bulk-rock major- and trace-element geochemistry of Site $\mathbf{9 2 1}$ mafic plutonic samples.

\begin{tabular}{|c|c|c|c|c|c|c|c|c|c|}
\hline Hole: & $921 \mathrm{~A}$ & $921 \mathrm{~B}$ & $921 \mathrm{~B}$ & $921 \mathrm{~B}$ & $921 \mathrm{~B}$ & $921 \mathrm{~B}$ & $921 \mathrm{~B}$ & $921 \mathrm{~B}$ & $921 \mathrm{~B}$ \\
\hline Core, section, interval $(\mathrm{cm})$ : & $2 \mathrm{R}-1,34-40$ & $1 \mathrm{~W}-1,64-70$ & IW-2, 5-8 & $2 \mathrm{R}-1,85-88$ & $2 R-2,36-43$ & $3 \mathrm{R}-1,13-20$ & $3 \mathrm{R}-1,80-84$ & $3 R-1,133-13$ & $3 R-2,43-46$ \\
\hline Depth (mbsf): & 8.04 & 0.64 & 1.47 & 15.45 & 16.28 & 24.63 & 25.30 & 25.83 & 26.40 \\
\hline Laboratory (major elements): & ODP XRF & ODP XRF & ODP XRF & ODP XRF & ICP-AES-UH & ODP XRF & ICP-AES-UH & ODP XRF & ICP-AES-UH \\
\hline Laboratory (trace and REEs): & ICP-AES-UH & ICP-AES-UH & ICP-AES-UH & ICP-AES-UH & ICP-AES-UH & ICP-AES-UH & ICP-AES-UH & ICP-AES-UH & ICP-AES-UH \\
\hline Lithology: & Olivine gabbro & MGabbro & Diabase & Gabbro & Olivine gabbro & $\begin{array}{c}\text { Olivine } \\
\text { MGabbro }\end{array}$ & Olivine gabbro & Gabbro & Gabbro \\
\hline \multicolumn{10}{|l|}{ Major elements (wt\%) } \\
\hline $\mathrm{SiO}_{2}$ & 51.21 & 51.25 & 49.08 & 49.58 & 51.01 & 50.69 & 49.69 & 52.70 & 50.64 \\
\hline $\mathrm{TiO}_{2}$ & 0.36 & 0.47 & 1.38 & 0.35 & 0.39 & 0.37 & 1.21 & 0.45 & 0.30 \\
\hline $\mathrm{Al}_{2} \mathrm{O}_{3}$ & 16.71 & 17.19 & 16.96 & 14.81 & 17.40 & 16.48 & 16.24 & 15.41 & 17.20 \\
\hline $\mathrm{Fe}_{2} \mathrm{O}_{3}$ & 6.42 & 6.17 & 9.39 & 9.11 & 5.80 & 5.81 & 9.86 & 5.78 & 6.73 \\
\hline $\mathrm{MnO}^{3}$ & 0.13 & 0.14 & 0.18 & 0.15 & 0.11 & 0.12 & 0.17 & 0.13 & 0.12 \\
\hline $\mathrm{MgO}$ & 9.67 & 9.09 & 7.99 & 12.45 & 8.50 & 10.23 & 8.14 & 9.07 & 10.24 \\
\hline $\mathrm{CaO}$ & 13.64 & 11.96 & 11.86 & 10.87 & 13.35 & 13.92 & 12.12 & 14.28 & 12.91 \\
\hline $\mathrm{Na}_{2} \mathrm{O}$ & 2.38 & 2.90 & 2.58 & 2.83 & 2.84 & 2.12 & 2.87 & 2.41 & 2.54 \\
\hline $\mathrm{K}_{2} \mathrm{O}$ & 0.01 & 0.03 & 0.01 & 0.05 & 0.01 & 0.01 & 0.01 & 0.02 & 0.06 \\
\hline $\mathrm{P}_{2} \mathrm{O}_{5}$ & 0.00 & 0.04 & 0.08 & 0.04 & 0.02 & 0.00 & 0.08 & 0.00 & 0.02 \\
\hline $\mathrm{Cr}_{2} \mathrm{O}_{3}$ & 0.04 & 0.01 & 0.04 & 0.01 & 0.02 & 0.07 & 0.06 & 0.01 & 0.04 \\
\hline $\mathrm{NiO}$ & 0.01 & 0.01 & 0.01 & 0.02 & 0.01 & 0.02 & 0.01 & 0.01 & 0.01 \\
\hline Total & 100.58 & 99.26 & 99.57 & 100.28 & 99.46 & 99.84 & 100.46 & 100.27 & 100.82 \\
\hline LOI & 0.18 & 1.17 & 1.93 & 0.17 & 0.68 & 0.20 & 0.23 & 0.15 & 1.00 \\
\hline $\mathrm{CO}_{2}$ & 0.06 & 0.06 & 0.01 & 0.04 & & 0.06 & & 0.00 & \\
\hline $\mathrm{H}_{2} \mathrm{O}$ & 1.23 & 1.91 & 2.57 & 0.88 & & 0.68 & & 0.61 & \\
\hline FeOT & 5.78 & 5.55 & 8.45 & 8.20 & 5.22 & 5.23 & 8.87 & 5.20 & 6.06 \\
\hline \multicolumn{10}{|l|}{ Trace elements (ppm) } \\
\hline V & 139 & 121 & 203 & 128 & 185 & 149 & 294 & 196 & 130 \\
\hline $\mathrm{Ti}$ & 2158 & 2818 & 8273 & 2102 & 2338 & 2218 & 7254 & 2698 & 1824 \\
\hline $\mathrm{Cr}$ & 284 & 95 & 274 & 99 & 147 & 491 & 420 & 81 & 278 \\
\hline $\mathrm{Ni}$ & 103 & 83 & 118 & 127 & 103 & 163 & 99 & 67 & 113 \\
\hline $\mathrm{Sr}$ & 131 & 139 & 131 & 140 & 166 & 129 & 163 & 120 & 136 \\
\hline $\mathrm{Zr}$ & 21 & 58 & 89 & 29 & 28 & 20 & 46 & 19 & 17 \\
\hline $\mathrm{Ba}$ & 14 & 15 & 13 & 15 & 21 & 11 & 22 & 12 & 9 \\
\hline Co & 37 & 29 & 54 & 58 & & 38 & & 31 & \\
\hline $\mathrm{Cu}$ & 93 & 22 & 62 & 87 & & 107 & & 80 & \\
\hline $\mathrm{Zn}$ & 28 & 36 & 50 & 48 & & 28 & & 21 & \\
\hline $\mathrm{Sc}$ & 41 & 38 & 36 & 31 & 39 & 40 & 36 & 54 & 35 \\
\hline $\mathrm{Y}$ & 11 & 28 & 30 & 11 & 12 & 12 & 21 & 14 & 9 \\
\hline \multicolumn{10}{|l|}{ Rare earths (ppm) } \\
\hline $\mathrm{La}$ & 0.76 & 2.94 & 2.72 & 1.09 & 0.88 & 0.83 & 1.41 & 0.73 & 0.41 \\
\hline $\mathrm{Ce}$ & 2.93 & 10.13 & 9.69 & 4.01 & 3.70 & 3.17 & 5.64 & 2.83 & 1.78 \\
\hline Nd & 2.16 & 8.83 & 8.86 & 2.65 & 2.62 & 2.47 & 4.78 & 2.25 & 1.57 \\
\hline $\mathrm{Sm}$ & 0.84 & 2.84 & 2.95 & 0.96 & 0.98 & 0.95 & 1.78 & 0.96 & 0.62 \\
\hline Eu & 0.55 & 0.98 & 1.21 & 0.50 & 0.55 & 0.52 & 1.11 & 0.60 & 0.46 \\
\hline Gd & 1.34 & 3.76 & 4.03 & 1.43 & 1.51 & 1.51 & 2.73 & 1.68 & 1.00 \\
\hline Dy & 1.76 & 4.50 & 4.83 & 1.77 & 1.88 & 1.94 & 3.37 & 2.25 & 1.46 \\
\hline $\mathrm{Er}$ & 1.14 & 2.86 & 3.04 & 1.12 & 1.21 & 1.21 & 2.21 & 1.46 & 0.88 \\
\hline $\mathrm{Yb}$ & 1.01 & 2.65 & 2.77 & 1.08 & 1.14 & 1.11 & 2.04 & 1.29 & 0.77 \\
\hline $\mathrm{Lu}$ & 0.16 & 0.43 & 0.44 & 0.16 & 0.15 & 0.17 & 0.30 & 0.21 & \\
\hline $\begin{array}{l}\text { Isotopes } \\
{ }^{87} \mathrm{Sr} /{ }^{86} \mathrm{Sr}\end{array}$ & & & & & & & & & \\
\hline
\end{tabular}

Notes: MGabbro = microgabbro. Other abbreviations are defined in text and Table 2.

the mantle subsequent to melt extraction. The interpretation of most dunites within residual mantle sections as ultradepleted residuum based on visual inspection may also prove to be in error if lower forsterite values $(90-80)$ for olivine can be documented as a general phenomenon in oceanic mantle dunites. The zoned dunite-wehrlite-clinopyroxenite-gabbro intrusive rocks within mantle zones in Holes 920B and 920D that are described below, in Cannat, Karson, Miller, et al. (1995), and in Cannat, Chatin, et al. (this volume) exhibit these lower forsterite values.

The compositional and lithologic gaps between residual peridotite and gabbro appear to be completely filled by intrusive samples obtained in Holes 920B and 920D in the MARK region. Clinopyroxene, olivine, and orthopyroxene compositional fields measured during this study for peridotites and gabbros are also plotted in Figure 7A for comparison to the bulk chemistry of MARK peridotites and gabbros. These phases likewise show a continuum of mineral compositions without gaps (in terms of their $\mathrm{Mg \# s}$ ) between those characteristic of residual mantle and MORB basalts and gabbros.

\section{BULK-ROCK AND MINERAL CHEMISTRY OF ULTRAMAFIC AND COARSE-GRAINED INTRUSIVE ROCKS FROM HOLES 920B AND 920D}

Drilling into the peridotite massif on the western wall of the rift valley in the MARK region penetrated 126.4 meters below seafloor (mbsf) at Hole $920 \mathrm{~B}$ and resulted in $44.57 \mathrm{~m}$ of recovery. Penetration of 200.8 mbsf was achieved at Hole 920D and resulted in $95.08 \mathrm{~m}$ of recovery. Holes $920 \mathrm{~B}$ and $920 \mathrm{D}$ are only $20 \mathrm{~m}$ apart and overlap in recovery intervals. Many of the features in the core are correlatable (Shipboard Scientific Party, 1995). Lithologies within these holes included dominantly serpentinized residual harzburgite, but also minor amounts of variably altered intrusive clinopyroxenites, lherzolite, websterites, wehrlites, dunites, gabbro, oxide metagabbro, amphibolites, and diabases. Core recovery intervals and units identified in Holes 920B and 920D are shown in Figure 2A. Petrographic and core descriptions of these various lithologies are presented in Cannat, Karson, Miller, et al. (1995) and will not be reviewed here. 
Table 3 (continued).

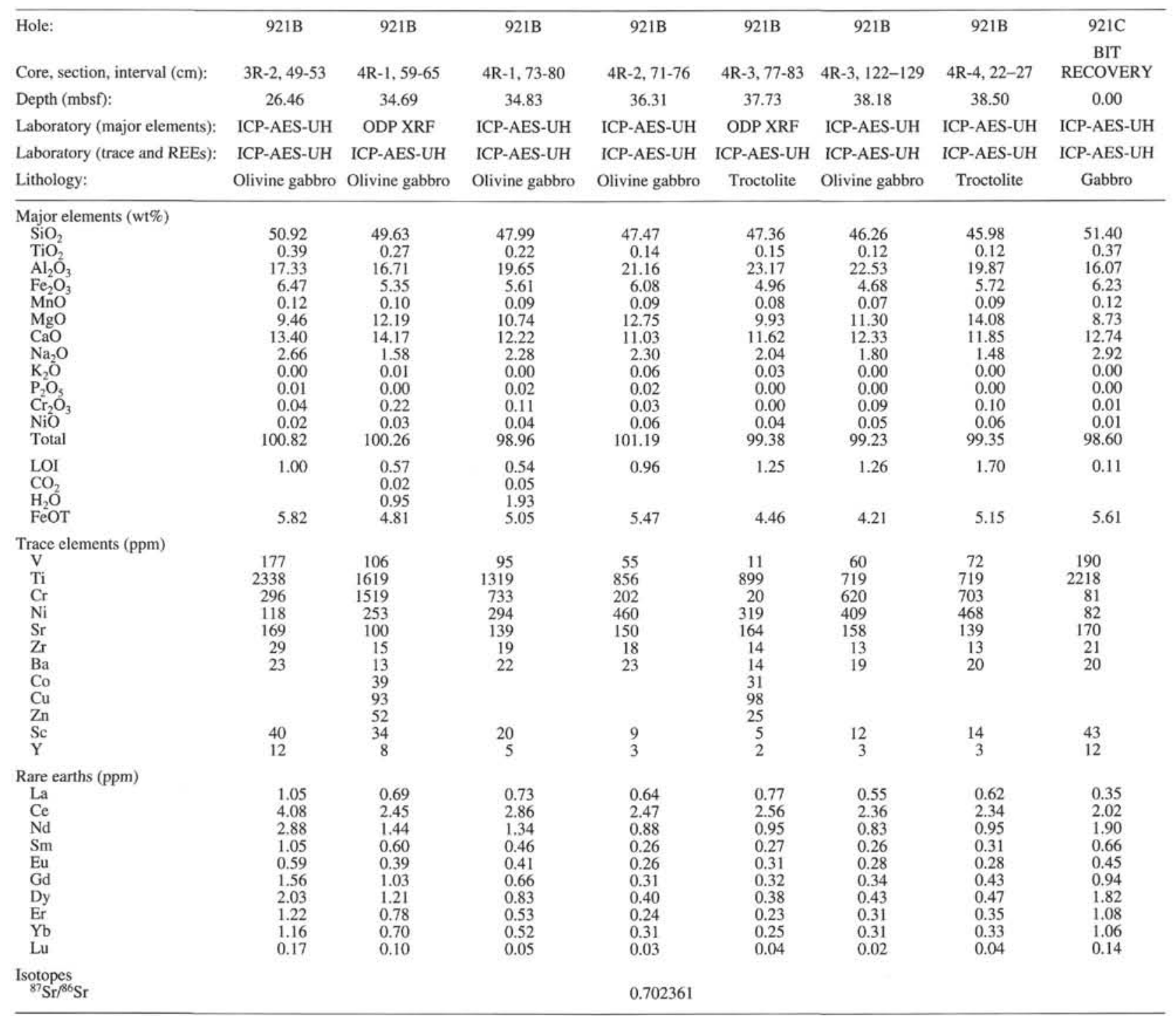

Residual harzburgites from these holes tend to be depleted in clinopyroxene. The average olivine: orthopyroxene: clinopyroxene: spinel mode is $80.5 \%: 17.6 \%: 2.5 \%: 1.1 \%$ for Hole $920 \mathrm{~B}$ harzburgites, and is $83.2 \%: 14.6 \%: 02.2 \%: 01.1 \%$ for Hole 920D (Shipboard Scientific Party, 1995). Bulk-rock chemistries for Hole 920B and 920D are reported in Tables 1 and 2, respectively, and samples are listed in order of their depth of recovery for each hole. The position of each sample in the core is indicated by its depth of recovery and can be correlated with the recovery interval of each lithologic unit shown in Figure 2A. Lithologies (i.e., their protoliths before serpentinization) are listed with each sample analysis. Analyses of diabasic rocks from Holes 920B and 920D are reported in Tables 1 and 2, but are discussed below more fully along with other diabasic rocks sampled during Leg 153 (also see Kempton and Casey, this volume).

\section{Effects of Alteration on Bulk Chemistry of the Peridotites}

The metasomatic effects of extensive alteration must be considered in any discussion of bulk chemistry of serpentinized mantle rocks. Although most samples from Holes 920B and 920D are strongly serpentinized (generally $80 \%-100 \%$ ) under greenschist facies conditions, these samples have not been subjected to the much stronger alteration effects caused by higher water/rock ratios that characterize the seafloor weathering zone. Because most previously analyzed samples of abyssal peridotites were recovered by dredging and submersibles, they have undergone some extent of seafloor weathering. Significant bulk-rock changes and elemental mobility have certainly been documented in samples affected by seafloor weathering, especially loss of bulk MgO (Snow and Dick, 1995) and gain in $\mathrm{CaO}$ or $\mathrm{CO}_{2}$ as a consequence of carbonate precipitation (Bonatti et al., 1980, 1984; Menzies et al., 1993). MgO loss by dissolution has been shown to be more significant in weathering rinds of some peridotite samples, but these processes postdate greenschist facies serpentinization and appear to represent a near-surface weathering phenomenon (Snow and Dick, 1995). This has an overall effect of lowering the $\mathrm{MgO} / \mathrm{SiO}_{2}$ ratio of most abyssal peridotites when compared to continental mantle xenoliths. In addition to $\mathrm{MgO}$ variations, extensive seafloor weathering and related high water/rock ra- 
Table 3 (continued).

\begin{tabular}{|c|c|c|c|c|c|c|c|c|c|}
\hline Hole: & $921 \mathrm{C}$ & $921 \mathrm{C}$ & $921 \mathrm{C}$ & $921 \mathrm{C}$ & $921 \mathrm{C}$ & $921 \mathrm{D}$ & 921D & $921 \mathrm{D}$ & $921 \mathrm{D}$ \\
\hline Core, section, interval $(\mathrm{cm})$ : & $2 \mathrm{R}-1,3-7$ & $2 \mathrm{R}-1,110-11$ & $2 R-2,114-12$ & $3 \mathrm{R}-1,83-89$ & $3 R-3,20-28$ & $2 \mathrm{R}-1,55-60$ & $4 \mathrm{R}-1,36-43$ & $4 \mathrm{R}-1,54-59$ & $4 \mathrm{R}-1,59-63$ \\
\hline Depth (mbsf): & 10.03 & 11.10 & 12.54 & 20.43 & 22.66 & 10.35 & 29.66 & 29.84 & 29.89 \\
\hline Laboratory (major elements): & ODP XRF & ODP XRF & ODP XRF & ODP XRF & ICP-AES-UH & ODP XRF & ICP-AES-UH & ODP XRF & ICP-AES-UH \\
\hline Laboratory (trace and REEs): & ICP-AES-UH & ICP-AES-UH & ICP-AES-UH & ICP-AES-UH & ICP-AES-UH & ICP-AES-UH & ICP-AES-UH & ICP-AES-UH & ICP-AES-UH \\
\hline Lithology: & Diabase & Olivine gabbro & Olivine gabbro & Olivine gabbro & Olivine gabbro & Gabbronorite & Gabbro & Olivine gabbro & Gabbro \\
\hline \multicolumn{10}{|l|}{ Major elements (wt\%) } \\
\hline $\begin{array}{l}\mathrm{SiO}_{2} \\
\mathrm{TiO}_{2} \\
\mathrm{Al}_{2} \mathrm{O}_{3} \\
\mathrm{Fe}_{2} \mathrm{O}_{3} \\
\mathrm{MnO} \\
\mathrm{MgO} \\
\mathrm{CaO} \\
\mathrm{Na}_{2} \mathrm{O} \\
\mathrm{K}_{2} \mathrm{O} \\
\mathrm{P}_{2} \mathrm{O}_{5} \\
\mathrm{CR}_{2} \mathrm{O}_{3} \\
\mathrm{NiO} \\
\text { Total }\end{array}$ & $\begin{array}{r}50.19 \\
1.31 \\
16.89 \\
9.49 \\
0.15 \\
7.61 \\
11.80 \\
2.69 \\
0.02 \\
0.07 \\
0.04 \\
0.02 \\
100.28\end{array}$ & $\begin{array}{r}50.39 \\
0.39 \\
17.60 \\
6.62 \\
0.12 \\
9.60 \\
12.56 \\
2.27 \\
0.01 \\
0.01 \\
0.10 \\
0.01 \\
99.68\end{array}$ & $\begin{array}{r}50.01 \\
0.44 \\
16.70 \\
4.91 \\
0.10 \\
11.27 \\
15.22 \\
1.61 \\
0.00 \\
0.00 \\
0.25 \\
0.03 \\
100.54\end{array}$ & $\begin{array}{r}49.70 \\
0.39 \\
17.18 \\
4.60 \\
0.09 \\
10.68 \\
15.14 \\
1.57 \\
0.00 \\
0.00 \\
0.25 \\
0.02 \\
99.62\end{array}$ & $\begin{array}{r}49.27 \\
0.44 \\
16.94 \\
4.56 \\
0.09 \\
11.26 \\
14.99 \\
1.65 \\
0.00 \\
0.01 \\
0.30 \\
0.03 \\
99.55\end{array}$ & $\begin{array}{r}53.77 \\
0.50 \\
17.41 \\
7.20 \\
0.15 \\
7.42 \\
10.34 \\
3.23 \\
0.03 \\
0.00 \\
0.01 \\
0.01 \\
100.06\end{array}$ & $\begin{array}{r}48.28 \\
1.83 \\
15.12 \\
10.71 \\
0.18 \\
8.35 \\
11.43 \\
3.00 \\
0.01 \\
0.00 \\
0.03 \\
0.01 \\
98.95\end{array}$ & $\begin{array}{r}48.56 \\
3.41 \\
11.85 \\
13.21 \\
0.25 \\
8.00 \\
12.17 \\
2.32 \\
0.02 \\
0.04 \\
0.02 \\
0.02 \\
99.86\end{array}$ & $\begin{array}{r}45.37 \\
4.28 \\
11.48 \\
15.32 \\
0.26 \\
8.95 \\
12.34 \\
2.19 \\
0.05 \\
0.57 \\
0.03 \\
0.01 \\
100.86\end{array}$ \\
\hline $\begin{array}{l}\mathrm{LOI} \\
\mathrm{CO}_{2} \\
\mathrm{H}_{2} \mathrm{O} \\
\text { FeOT }\end{array}$ & $\begin{array}{l}1.41 \\
0.00 \\
2.19 \\
8.54\end{array}$ & $\begin{array}{l}0.73 \\
0.00 \\
1.34 \\
5.96\end{array}$ & $\begin{array}{l}0.58 \\
0.00 \\
0.99 \\
4.42\end{array}$ & $\begin{array}{l}0.43 \\
0.03 \\
0.34 \\
4.14\end{array}$ & 0.99 & $\begin{array}{l}0.30 \\
0.00 \\
0.00 \\
6.48\end{array}$ & 1.61 & $\begin{array}{r}0.79 \\
0.00 \\
2.87 \\
11.89\end{array}$ & 2.01 \\
\hline $\begin{array}{l}\text { Trace elements (ppm) } \\
\text { V } \\
\mathrm{Ti} \\
\mathrm{Cr} \\
\mathrm{Ni} \\
\mathrm{Sr} \\
\mathrm{Zr} \\
\mathrm{Ba} \\
\mathrm{Co} \\
\mathrm{Cu} \\
\mathrm{Zn} \\
\mathrm{Sc} \\
\mathrm{Y}\end{array}$ & $\begin{array}{r}218 \\
7853 \\
280 \\
122 \\
137 \\
87 \\
14 \\
55 \\
74 \\
56 \\
35 \\
30\end{array}$ & $\begin{array}{r}125 \\
2338 \\
674 \\
114 \\
139 \\
24 \\
14 \\
34 \\
92 \\
35 \\
32 \\
11\end{array}$ & $\begin{array}{r}140 \\
2638 \\
1744 \\
204 \\
123 \\
23 \\
16 \\
44 \\
84 \\
25 \\
40 \\
11\end{array}$ & $\begin{array}{r}123 \\
2338 \\
1692 \\
184 \\
116 \\
22 \\
13 \\
24 \\
74 \\
21 \\
39 \\
10\end{array}$ & $\begin{array}{r}152 \\
2638 \\
2082 \\
250 \\
116 \\
30 \\
17\end{array}$ & $\begin{array}{r}134 \\
2998 \\
48 \\
43 \\
160 \\
23 \\
13 \\
23 \\
27 \\
42 \\
34 \\
13\end{array}$ & $\begin{array}{r}313 \\
10971 \\
195 \\
103 \\
145 \\
42 \\
20\end{array}$ & $\begin{array}{r}407 \\
20443 \\
104 \\
148 \\
119 \\
89 \\
12 \\
72 \\
128 \\
70 \\
53 \\
40\end{array}$ & $\begin{array}{r}509 \\
25675 \\
218 \\
84 \\
96 \\
98 \\
9\end{array}$ \\
\hline $\begin{array}{l}\text { Rare earths (ppm) } \\
\text { La } \\
\mathrm{Ce} \\
\mathrm{Nd} \\
\mathrm{Sm} \\
\mathrm{Eu} \\
\mathrm{Gd} \\
\mathrm{Dy} \\
\mathrm{Er} \\
\mathrm{Yb} \\
\mathrm{Lu}\end{array}$ & $\begin{array}{l}3.03 \\
9.73 \\
8.84 \\
2.92 \\
1.19 \\
3.97 \\
4.69 \\
2.99 \\
2.77 \\
0.44\end{array}$ & $\begin{array}{l}0.93 \\
3.45 \\
2.37 \\
0.89 \\
0.55 \\
1.38 \\
1.72 \\
1.08 \\
0.97 \\
0.14\end{array}$ & $\begin{array}{l}0.86 \\
3.22 \\
2.43 \\
0.95 \\
0.48 \\
1.52 \\
1.80 \\
1.14 \\
1.01 \\
0.15\end{array}$ & $\begin{array}{l}0.93 \\
3.19 \\
2.42 \\
0.92 \\
0.49 \\
1.43 \\
1.70 \\
1.07 \\
0.94 \\
0.15\end{array}$ & $\begin{array}{l}1.09 \\
3.76 \\
2.74 \\
1.02 \\
0.48 \\
1.55 \\
1.81 \\
1.19 \\
1.04 \\
0.15\end{array}$ & $\begin{array}{l}1.40 \\
3.58 \\
2.77 \\
1.06 \\
0.81 \\
1.68 \\
2.17 \\
1.43 \\
1.34 \\
0.22\end{array}$ & $\begin{array}{l}1.27 \\
4.82 \\
4.23 \\
1.54 \\
1.09 \\
2.29 \\
3.20 \\
2.15 \\
2.08 \\
0.33\end{array}$ & $\begin{array}{l}1.97 \\
7.54 \\
8.41 \\
3.26 \\
1.49 \\
5.19 \\
6.53 \\
4.15 \\
3.83 \\
0.63\end{array}$ & $\begin{array}{c}3.17 \\
12.9 \\
15.0 \\
5.31 \\
2.08 \\
7.71 \\
8.95 \\
5.38 \\
4.40\end{array}$ \\
\hline $\begin{array}{l}\text { Isotopes } \\
{ }^{87} \mathrm{Sr} /{ }^{86} \mathrm{Sr}\end{array}$ & & \multicolumn{7}{|c|}{ Isotopes } & \\
\hline
\end{tabular}

tios during weathering can also affect other major and trace elements in some cases (Menzies et al., 1993). Because most analyses available for abyssal peridotites represent surface weathered samples that tend to be strongly altered, the bulk chemical compositions have often been strongly criticized or disregarded as representative of mantle compositions.

Because of this, abyssal peridotite compositions have generally been calculated on the basis of mineral chemistry and primary modal abundances (Dick, 1989; Snow and Dick, 1995) as a way around the problem of element mobility during seafloor weathering and alteration. This technique, however, has some drawbacks as well. Determining modal abundances of primary minerals is at best difficult in highly altered and serpentinized rocks, and there is no statistical means to assess the accuracy and quality of the modal estimates because of the various states of alteration. Interpreting the mode of a sample likewise requires that a choice be made among assuming constant volume (loss of mass), constant mass (isochemical except for $\mathrm{H}_{2} \mathrm{O}$ addition), or variations in both mass and volume during alteration (Komor et al., 1985b). Primary accessory phases may also not to be totally accounted for when calculating bulk compositions from modes.

If it can be assumed that samples taken from deeper than the weathering zone and outside major shear zones are less likely to have undergone high water/rock ratios and attendant large changes in bulk chemistry, the bulk analyses of drill core may provide an alternative way to assess depleted abyssal mantle compositions. In support of this contention, although water/rock ratios have been estimated to be between $10^{3}$ and $10^{5}$ for abyssal peridotites affected by seafloor weathering (Snow and Dick, 1995), water/rock ratios during greenschist facies serpentinization in samples not affected by seafloor weathering are generally regarded to be much smaller (6-30), based on both experimental and empirical data sets (Janecky, 1982; Janecky and Seyfried, 1983, 1986; Silant'ev, 1995; Menzies et al., 1993). Although interpretive problems can arise in bulk analyses if large hydrothermal veins (e.g., large serpentine or aragonite veins) or thick serpentine shear zones that are likely to have undergone high water/rock ratios are included in the sample chosen for analysis, these problems can be eliminated or minimized in most samples by careful removal of large veins and avoidance of shear zones affected by extreme hydration. This is not to say that there are not wholesale changes in mineralogy of the samples or that the bulk-rock effects of serpentinization can be ignored. To some extent, even at depths below the seafloor weathering zone, the bulk-rock geochemistries of all oceanic peridotites are affected by serpentinization, simply by the addition of volatiles (up to $\sim 16 \% \mathrm{H}_{2} \mathrm{O}$ in Leg 153 peridotites). Major, as well as trace elements, are analyzed on a volatile-free basis to 
Table 3 (continued).

\begin{tabular}{|c|c|c|c|c|c|c|c|c|c|c|}
\hline Hole: & $921 \mathrm{D}$ & $921 \mathrm{D}$ & 921D & $921 \mathrm{D}$ & $921 \mathrm{E}$ & $921 \mathrm{E}$ & $921 \mathrm{E}$ & $921 \mathrm{E}$ & $921 \mathrm{E}$ & $921 \mathrm{E}$ \\
\hline Core, section, interval $(\mathrm{cm})$ : & $5 \mathrm{R}-1,118-12$ & $5 R-2,47-51$ & $5 R-2,56-63$ & $5 R-2,68-73$ & 2R-1, 34-39 & $2 \mathrm{R}-2,24-30$ & $2 \mathrm{R}-2,87-93$ & $3 R-1,66-72$ & $3 R-1,138-14$ & $4 \mathrm{R}-1,18-24$ \\
\hline Depth (mbsf): & 40.08 & 40.82 & 40.91 & 41.03 & 10.24 & 11.39 & 12.02 & 20.46 & 21.18 & 29.88 \\
\hline Laboratory (major elements): & ODP XRF & ICP-AES-UH & ODP XRF & ICP-AES-UH & ICP-AES-UH & ODP XRF & ICP-AES-UH & ICP-AES-UH & ODP XRF & ICP-AES-UH \\
\hline Laboratory (trace and REEs): & ICP-AES-UH & ICP-AES-UH & ICP-AES-UH & ICP-AES-UH & ICP-AES-UH & ICP-AES-UH & ICP-AES-UH & ICP-AES-UH & ICP-AES-UH & ICP-AES-UH \\
\hline Lithology: & Olivine gabbro & Olivine gabbro & Olivine gabbro & Gabbro & $\begin{array}{c}\text { Gabbro, } \\
\text { with veins }\end{array}$ & Olivine gabbro & Gabbro & Gabbro & Olivine gabbro & Gabbro \\
\hline \multicolumn{11}{|l|}{ Major elements (wt\%) } \\
\hline $\mathrm{SiO}_{2}$ & 51.17 & 47.60 & 47.01 & 50.53 & 51.28 & 50.69 & 48.91 & 51.10 & 51.00 & 51.66 \\
\hline $\mathrm{TiO}_{2}^{2}$ & 0.33 & 0.20 & 0.20 & 0.29 & 0.52 & 0.61 & 0.34 & 0.36 & 0.41 & 0.29 \\
\hline $\mathrm{Al}_{2} \mathrm{O}_{3}$ & 17.15 & 21.17 & 20.03 & 17.47 & 15.99 & 15.37 & 17.07 & 16.68 & 17.42 & 20.76 \\
\hline $\mathrm{Fe}_{2} \mathrm{O}_{3}$ & 5.43 & 5.41 & 6.76 & 5.62 & 5.81 & 7.56 & 8.59 & 5.82 & 5.82 & 3.58 \\
\hline $\mathrm{MnO}$ & 0.11 & 0.08 & 0.11 & 0.10 & 0.12 & 0.14 & 0.14 & 0.11 & 0.12 & 0.07 \\
\hline $\mathrm{MgO}$ & 9.70 & 11.04 & 13.30 & 9.46 & 8.64 & 9.65 & 9.60 & 9.52 & 9.52 & 5.74 \\
\hline $\mathrm{CaO}$ & 13.37 & 11.53 & 10.11 & 13.62 & 14.16 & 13.18 & 11.15 & 12.38 & 13.43 & 13.88 \\
\hline $\mathrm{Na}_{2} \mathrm{O}$ & 2.25 & 2.27 & 1.95 & 2.38 & 2.77 & 2.25 & 3.00 & 2.74 & 2.44 & 3.22 \\
\hline $\mathrm{K}_{2} \mathrm{O}$ & 0.00 & 0.04 & 0.02 & 0.04 & 0.00 & 0.02 & 0.00 & 0.04 & 0.03 & 0.00 \\
\hline $\mathrm{P}_{2} \mathrm{O}_{5}$ & 0.00 & 0.01 & 0.00 & 0.03 & 0.01 & 0.01 & 0.00 & 0.02 & 0.00 & 0.00 \\
\hline $\mathrm{CR}_{2} \mathrm{O}_{3}$ & 0.03 & 0.03 & 0.00 & 0.13 & 0.03 & 0.03 & 0.02 & 0.07 & 0.05 & 0.05 \\
\hline $\mathrm{NiO}$ & 0.01 & 0.04 & 0.04 & 0.02 & 0.01 & 0.01 & 0.02 & 0.02 & 0.02 & 0.01 \\
\hline Total & 99.56 & 99.42 & 99.54 & 99.70 & 99.35 & 99.52 & 98.84 & 98.86 & 100.26 & 99.26 \\
\hline LOI & 0.85 & 1.64 & . 0.97 & 0.83 & 0.27 & 0.29 & 0.10 & 0.55 & 0.55 & 0.33 \\
\hline $\mathrm{CO}_{2}$ & 0.02 & & 0.02 & & & 0.00 & & & 0.03 & \\
\hline $\mathrm{H}_{2} \mathrm{O}$ & 1.21 & & 1.43 & & & 0.47 & & & 0.00 & \\
\hline FeOT & 4.89 & 4.87 & 6.08 & 5.05 & 5.23 & 6.80 & 7.73 & 5.24 & 5.24 & 3.22 \\
\hline \multicolumn{11}{|l|}{ Trace elements (ppm) } \\
\hline V & 119 & 55 & 37 & 120 & 206 & 167 & 124 & 147 & 163 & 140 \\
\hline $\mathrm{Ti}$ & 1978 & 1199 & 1199 & 1764 & 3117 & 3657 & 2038 & 2158 & 2458 & 1739 \\
\hline $\mathrm{Cr}$ & 220 & 209 & 32 & 882 & 232 & 187 & 152 & 489 & 321 & 325 \\
\hline $\mathrm{Ni}$ & 112 & 312 & 331 & 182 & 95 & 116 & 132 & 160 & 146 & 67 \\
\hline $\mathrm{Sr}$ & 148 & 162 & 147 & 126 & 138 & 128 & 155 & 134 & 142 & 194 \\
\hline $\mathrm{Zr}$ & 17 & 17 & 19 & 38 & 25 & 41 & 22 & 58 & 31 & 23 \\
\hline $\mathrm{Ba}$ & 13 & 20 & 14 & 9 & 17 & 14 & 18 & 19 & 11 & 20 \\
\hline $\mathrm{Co}$ & 35 & & 46 & & & 38 & & & 71 & \\
\hline $\mathrm{Cu}$ & 85 & & 89 & & & 118 & & & 55 & \\
\hline $\mathrm{Zn}$ & 24 & & 33 & & & 41 & & & 36 & \\
\hline Sc & 38 & 11 & 8 & 29 & 48 & 44 & 29 & 35 & 36 & 32 \\
\hline $\mathrm{Y}$ & 9 & 4 & 4 & 8 & 17 & 18 & 10 & 22 & 14 & 9 \\
\hline \multicolumn{11}{|l|}{ Rare earths (ppm) } \\
\hline $\mathrm{La}$ & 0.65 & 0.60 & 0.77 & 0.23 & 1.01 & 1.28 & 0.94 & 2.80 & 1.33 & 0.70 \\
\hline $\mathrm{Ce}$ & 22.46 & 2.44 & 2.71 & 1.77 & 4.05 & 4.65 & 3.68 & 9.09 & 4.88 & 3.02 \\
\hline $\mathrm{Nd}$ & 41.49 & 1.28 & 1.25 & 1.20 & 3.29 & 3.98 & 2.44 & 6.35 & 3.59 & 1.71 \\
\hline Sm & 0.63 & 0.28 & 0.41 & 0.49 & 1.27 & 1.46 & 0.87 & 2.03 & 1.26 & 0.65 \\
\hline Eu & 0.47 & 0.26 & 0.34 & 0.32 & 0.76 & 0.72 & 0.60 & 0.66 & 0.61 & 0.44 \\
\hline Gd & 1.11 & 0.26 & 0.56 & 0.83 & 2.03 & 2.19 & 1.28 & 2.67 & 1.81 & 1.02 \\
\hline Dy & 1.46 & 0.59 & 0.69 & 1.23 & 2.61 & 2.88 & 1.59 & 3.32 & 2.28 & 1.29 \\
\hline Er & 0.92 & 0.38 & 0.47 & 0.74 & 1.67 & 1.80 & 1.02 & 2.26 & 1.44 & 0.85 \\
\hline $\mathrm{Yb}$ & 0.82 & 0.39 & 0.43 & 0.65 & 1.47 & 1.64 & 0.96 & 2.18 & 1.35 & 0.80 \\
\hline $\mathrm{Lu}$ & 0.12 & 0.05 & 0.07 & & 0.22 & 0.25 & 0.14 & 0.33 & 0.21 & 0.09 \\
\hline \multicolumn{11}{|l|}{ Isotopes } \\
\hline${ }^{87} \mathrm{Sr} /{ }^{86} \mathrm{Sr}$ & & & & & & & & 0.702592 & & \\
\hline
\end{tabular}

eliminate the effects of volatile dilution, so analyses more closely approximate the preserpentinization composition. Because Leg 153 ultramafic rocks were drilled and sampled from below the seafloor weathering zone, major elements may be less likely to have been significantly affected or mobilized by the serpentinization or alteration processes. Various studies of unweathered samples that have undergone greenschist facies serpentinization show that the alteration can be regarded as near isochemical (i.e., except for the addition of volatiles; Komor et al., 1985b; Menzies et al., 1993).

In the seawater-peridotite serpentinization experiments by Seyfried and Dibble (1980) and Janecky and Seyfried (1986), the concentrations of $\mathrm{Na}$ and $\mathrm{K}$ in the altering fluids were constant during serpentinization, suggesting little exchange. Likewise, in the Leg 153 ultramafic samples analyzed here, there is no apparent alkali gain. $\mathrm{CaO}$ concentrations in the fluid of these experiments tended to decrease because of formation of anhydrite and possibly carbonate. Anhydrite was not observed in Leg 153 serpentinized peridotites, and only minor aragonite veining exists. Although bulk-rock $\mathrm{Sr}$ and $\mathrm{CaO}$ content and ${ }^{87} \mathrm{Sr}^{186} \mathrm{Sr}$ ratios can increase significantly if carbonate or other Ca-rich phases are formed in the sample (Menzies et al., 1993), the extent of aragonite veining is low in the Leg 153 samples and thought to represent local alteration of calcic primary phases and fixation of $\mathrm{CO}_{2}$. They are most commonly associated with zones where plagioclase or clinopyroxene-rich rocks are altered. Thus $\mathrm{CaO}, \mathrm{CO}_{2}$, and $\mathrm{Sr}$ abundances are typically low in the bulk-rock analyses of unweathered Leg 153 residual peridotites. Average $\mathrm{CaO}$ contents of $0.45 \mathrm{wt} \%$ in Hole 920B and $0.25 \mathrm{wt} \%$ in Hole 920D are similar to those expected based on an average harzburgite primary clinopyroxene mode of $2.5 \%$ in Hole $920 \mathrm{~B}$ and $2.2 \%$ in Hole 920D. This indicates that the extensive addition of carbonate minerals, which is observed in many weathered abyssal peridotite samples (e.g., Bonatti et al., 1980), is likely to be the result of near-surface seafloor weathering and precipitation from seawater (also see Bonatti et al., 1984; Snow and Dick, 1995) rather than the original greenschist facies serpentinization process. Fixation of $\mathrm{CO}_{2}$ in zones where Ca-rich primary phases are altered is likely to cause the formation of limited carbonate and carbonate veins observed in the core (Shipboard Scientific Party, 1995).

$\mathrm{MgO}$ also tended to decrease in the experimental solutions of Seyfried and Dibble (1980) and Janecky and Seyfried (1986) during greenschist facies serpentinization, suggesting that $\mathrm{MgO}$ addition from a seawater reservoir could be possible within peridotites. This should cause shifts to higher $\mathrm{MgO} / \mathrm{SiO}_{2}$ ratios when compared with unaltered depleted mantle xenoliths. These upward shifts are not apparent with the MARK peridotites. The $\mathrm{MgO}$ content of alteration seawater or hydrothermal fluid would be rapidly depleted during pas- 
Table 3 (continued).

\begin{tabular}{|c|c|c|c|c|c|}
\hline Hole: & $921 \mathrm{E}$ & $921 \mathrm{E}$ & $921 \mathrm{E}$ & $921 \mathrm{E}$ & $921 \mathrm{E}$ \\
\hline Core, section, interval $(\mathrm{cm})$ : & $4 \mathrm{R}-1,44-50$ & $5 R-1,0-6$ & $5 R-1,45-51$ & $5 R-2,32-39$ & $5 R-2,125-13$ \\
\hline Depth (mbsf): & 30.14 & 39.60 & 40.05 & 40.96 & 41.89 \\
\hline Laboratory (major elements): & ODP XRF I & ICP-AES-UH & ICP-AES-UH & ICP-AES-UH & ODP XRF \\
\hline Laboratory (trace and REEs): & ICP-AES-UH I & ICP-AES-UH & ICP-AES-UH & ICP-AES-UH & ICP-AES-UH \\
\hline Lithology: & Olivine gabbro & Diorite & Olivine gabbro & Gabbro & Olivine gabbro \\
\hline \multicolumn{6}{|l|}{ Major elements (wt\%) } \\
\hline $\mathrm{SiO}_{2}$ & 50.94 & 61.24 & 49.02 & 50.08 & 49.28 \\
\hline $\mathrm{TiO}_{2}$ & 0.28 & 0.79 & 0.31 & 0.27 & 0.21 \\
\hline $\mathrm{Al}_{2} \mathrm{O}_{3}$ & 20.63 & 18.51 & 15.98 & 16.15 & 21.72 \\
\hline $\mathrm{Fe}_{2} \mathrm{O}_{3}$ & 4.54 & 5.33 & 6.23 & 5.42 & 5.31 \\
\hline $\mathrm{MnO}$ & 0.09 & 0.08 & 0.11 & 0.10 & 0.09 \\
\hline $\mathrm{MgO}$ & 7.72 & 1.62 & 12.33 & 12.87 & 9.22 \\
\hline $\mathrm{CaO}$ & 12.88 & 5.15 & 12.78 & 13.64 & 11.34 \\
\hline $\mathrm{Na}_{2} \mathrm{O}$ & 2.63 & 6.58 & 2.10 & 2.13 & 2.63 \\
\hline $\mathrm{K}_{2} \mathrm{O}$ & 0.02 & 0.20 & 0.00 & 0.05 & 0.01 \\
\hline $\mathrm{P}_{2} \mathrm{O}_{5}$ & 0.00 & 0.13 & 0.02 & 0.02 & 0.00 \\
\hline $\mathrm{CR}_{2} \mathrm{O}_{3}$ & 0.03 & 0.00 & 0.08 & 0.10 & 0.01 \\
\hline $\mathrm{NiO}^{3}$ & 0.02 & 0.00 & 0.03 & 0.03 & 0.02 \\
\hline Total & 99.78 & 99.63 & 98.99 & 100.86 & 99.84 \\
\hline LOI & 0.55 & 1.56 & 0.86 & 0.93 & 0.53 \\
\hline $\mathrm{CO}_{2}$ & 0.03 & & & & 0.04 \\
\hline $\mathrm{H}_{2} \mathrm{O}$ & 0.00 & & & & 1.18 \\
\hline FeOT & 4.09 & 4.80 & 5.61 & 4.88 & 4.78 \\
\hline \multicolumn{6}{|l|}{ Trace elements (ppm) } \\
\hline V & 89 & 62 & 137 & 150 & 34 \\
\hline $\mathrm{Ti}$ & 1679 & 4729 & 1858 & 1632 & 1259 \\
\hline $\mathrm{Cr}$ & 214 & 20 & 529 & 695 & 79 \\
\hline $\mathrm{Ni}$ & 121 & 13 & 261 & 269 & 158 \\
\hline $\mathrm{Sr}$ & 154 & 168 & 130 & 123 & 174 \\
\hline $\mathrm{Zr}$ & 34 & 296 & 27 & 22 & 15 \\
\hline $\mathrm{Ba}$ & 10 & 31 & 18 & 19 & 13 \\
\hline Co & 36 & & & & 32 \\
\hline $\mathrm{Cu}$ & 65 & & & & 51 \\
\hline $\mathrm{Zn}$ & 29 & & & & 23 \\
\hline $\mathrm{Sc}$ & 22 & 7 & 33 & 36 & 11 \\
\hline $\mathrm{Y}$ & 10 & 93 & 8 & 8 & 4 \\
\hline \multicolumn{6}{|l|}{ Rare earths (ppm) } \\
\hline La & 1.27 & 11.48 & 0.79 & 0.74 & 0.64 \\
\hline $\mathrm{Ce}$ & 4.33 & 34.37 & 3.16 & 2.93 & 2.48 \\
\hline Nd & 2.72 & 28.07 & 1.95 & 1.84 & 0.94 \\
\hline Sm & 0.91 & 8.82 & 0.71 & 0.69 & 0.33 \\
\hline $\mathrm{Eu}$ & 0.52 & 5.36 & 0.45 & 0.42 & 0.36 \\
\hline Gd & 1.24 & 11.38 & 1.06 & 1.07 & 0.46 \\
\hline Dy & 1.57 & 14.02 & 1.28 & 1.27 & 0.63 \\
\hline $\mathrm{Er}$ & 1.01 & 9.79 & 0.82 & 0.82 & 0.39 \\
\hline $\mathrm{Yb}$ & 0.95 & 9.43 & 0.78 & 0.76 & 0.38 \\
\hline $\mathrm{Lu}$ & 0.14 & & 0.10 & 0.10 & 0.04 \\
\hline $\begin{array}{l}\text { Isotopes } \\
{ }^{87} \mathrm{Sr} /{ }^{86} \mathrm{Sr}\end{array}$ & & & & & \\
\hline
\end{tabular}

sage through the near-surface mafic or peridotite layers (Bischoff and Seyfried, 1978; Campbell et al., 1988), so that alteration at depth by hydrothermal fluids would produce minimal $\mathrm{MgO}$ gain from a seawater reservoir. Such low-MgO hydrothermal solutions are likely to be undersaturated with respect to the rock, accounting for some mobility of $\mathrm{MgO}$ and possibly small localized losses or gains (e.g., Komor et al., 1985b). Given the low water/rock ratios thought to be operative during serpentinization, small $\mathrm{MgO}$ additions or extractions from the peridotite are probable, but major shifts are unlikely to significantly affect the $\mathrm{MgO}$ content of the peridotites (ranging from $\sim 40$ to $44 \mathrm{wt} \%$ ) unless associated with zones of high fluid-rock ratios (e.g., along shear zones and large hydrothermal veins).

The extent of $\mathrm{MgO}$ addition or extraction may be evaluated in the samples analyzed. There are strong similarities between the wholerock $\mathrm{Mg} \# \mathrm{~s}\left(100 \times \mathrm{Mg} /\left[\mathrm{Mg}+\mathrm{Fe}^{*}\right]\right)$ and the $\mathrm{Mg} \# \mathrm{~s}$ of mafic mineral phases analyzed in the same peridotites. There is also a near constancy of bulk-rock Mg\#s in the residual harzburgites, as expected of residual mantle if metasomatism is not significant. In addition, $\mathrm{MgO}$ and $\mathrm{MgO} / \mathrm{SiO}_{2}$ ratios are similar to those of unaltered depleted mantle xenoliths (Jagoutz et al., 1979; Hart and Zindler, 1986). They are also similar to those of residual mantle of ophiolites, where studies of serpentinization show that it was largely constant mass and isochemical (i.e., except for the addition of water; Komor et al., 1985b). Serpentinized harzburgites that compositionally approximate mass balance typically contain brucite in addition to serpentine (Helmley et al., 1977; Komor et al., 1985b; Lane, 1988; Gillis et al., 1993; Snow and Dick, 1995). Brucite is also observed in the drilled peridotites of Leg 153 (Shipboard Scientific Party, 1995). Finally, the $\mathrm{MgO} / \mathrm{SiO}_{2}$ of MARK residual peridotites, mantle xenoliths, and ophiolite massifs are somewhat higher and lie within the same ranges when compared with most bulk-rock data from weathered abyssal peridotites, which show lower $\mathrm{MgO} / \mathrm{SiO}_{2}$ ratios. This appears to indicate that strong depletions or additions of $\mathrm{MgO}$ are unlikely for MARK peridotites, unlike their weathered ocean-floor counterparts.

$\mathrm{Al}_{2} \mathrm{O}_{3}$ and $\mathrm{TiO}_{2}$ are expected to be the most immobile major elements within serpentinized peridotites (Mottl, 1976; Nesbitt and Wilson, 1992; Snow and Dick, 1995). $\mathrm{Al}_{2} \mathrm{O}_{3}$ is generally regarded as insoluble in seawater at any temperature (Janecky and Seyfried, 1986). In this study, we rely heavily on the bulk $\mathrm{Al}_{2} \mathrm{O}_{3}$ content in estimations of the extent of mantle depletion because it is likely to have been relatively immobile. Trace elements such as Ni, Cr, REEs, and high field-strength elements (HFSEs) are also unlikely to be significantly altered (Menzies et al., 1993), except at very high water/rock ratios typical of the weathering zone, but this is atypical of deeper levels where greenschist facies alteration occurs.

The Leg 153 peridotite Holes 920B and 920D represent the deepest penetrations into unweathered oceanic residual mantle to date. Certain elements in the peridotites are likely to have been mobilized 
Table 4. Bulk-rock major- and trace-element geochemistry of Site 922 mafic plutonic samples.

\begin{tabular}{|c|c|c|c|c|c|c|c|c|}
\hline Hole: & $922 \mathrm{~A}$ & $922 \mathrm{~A}$ & $922 \mathrm{~A}$ & $922 \mathrm{~A}$ & $922 \mathrm{~A}$ & $922 \mathrm{~A}$ & $992 \mathrm{~A}$ & $922 \mathrm{~A}$ \\
\hline Core, interval $(\mathrm{cm})$ : & $1 R-1,130-133$ & $2 \mathrm{R}-1,83-87$ & $2 \mathrm{R}-1,122-12$ & $2 R-2,68-74$ & $2 \mathrm{R}-2,106-11$ & $2 R-5,85-90$ & 3R-1, 104-109 & $3 R-1,104-109$ \\
\hline Depth (mbsf): & 1.30 & 7.43 & 7.82 & 7.53 & 9.08 & 12.79 & 14.54 & 11.96 \\
\hline Laboratory (major elements) & ODP XRF & ICP-AES-UH & ODP XRF & ICP-AES-UH & ODP XRF & ICP-AES-UH & ODP XRF & ODP XRF \\
\hline Laboratory (trace and REEs) & ICP-AES-UH & ICP-AES-UH & ICP-AES-UH & ICP-AES-UH & ICP-AES-UH & ICP-AES-UH & ICP-AES-UH & ICP-AES-UH \\
\hline Lithology: & MOl-gabbro & Olivine gabbro & Troctolite & Olivine gabbro & Oxide gabbro & Oxide gabbro & Oxide gabbro & Olivine gabbro \\
\hline \multicolumn{9}{|l|}{ Major elements (wt\%) } \\
\hline $\begin{array}{l}\mathrm{SiO}_{2} \\
\mathrm{TiO}_{2} \\
\mathrm{Al}_{2} \mathrm{O}_{3} \\
\mathrm{Fe}_{2} \mathrm{O}_{3} \\
\mathrm{MnO} \\
\mathrm{MgO} \\
\mathrm{CaO} \\
\mathrm{Na}_{2} \mathrm{O} \\
\mathrm{K}_{2} \mathrm{O} \\
\mathrm{P}_{2} \mathrm{O}_{5} \\
\mathrm{CR}_{2} \mathrm{O}_{3} \\
\mathrm{NiO} \\
\text { Total }\end{array}$ & $\begin{array}{r}47.79 \\
0.27 \\
22.38 \\
5.14 \\
0.11 \\
11.81 \\
11.39 \\
1.70 \\
0.03 \\
0.00 \\
0.01 \\
0.04 \\
100.67\end{array}$ & $\begin{array}{r}45.76 \\
0.81 \\
17.44 \\
10.78 \\
0.16 \\
14.58 \\
9.19 \\
1.96 \\
0.05 \\
0.20 \\
0.02 \\
0.07 \\
101.01\end{array}$ & $\begin{array}{r}46.41 \\
0.19 \\
18.50 \\
6.15 \\
0.11 \\
17.05 \\
10.81 \\
1.29 \\
0.03 \\
0.01 \\
0.08 \\
0.07 \\
100.69\end{array}$ & $\begin{array}{r}45.77 \\
0.15 \\
21.12 \\
5.20 \\
0.08 \\
13.91 \\
10.83 \\
1.87 \\
0.00 \\
0.04 \\
0.02 \\
0.06 \\
99.05\end{array}$ & $\begin{array}{r}44.21 \\
2.06 \\
18.14 \\
13.17 \\
0.19 \\
7.26 \\
9.75 \\
2.54 \\
0.03 \\
1.50 \\
0.00 \\
0.03 \\
98.88\end{array}$ & $\begin{array}{r}44.15 \\
2.66 \\
14.34 \\
15.34 \\
0.24 \\
6.91 \\
11.69 \\
2.36 \\
0.05 \\
1.07 \\
0.08 \\
0.02 \\
98.91\end{array}$ & $\begin{array}{r}40.22 \\
5.14 \\
12.60 \\
18.57 \\
0.32 \\
6.80 \\
9.83 \\
2.25 \\
0.03 \\
3.35 \\
0.02 \\
0.01 \\
99.14\end{array}$ & $\begin{array}{r}47.59 \\
0.38 \\
21.50 \\
5.24 \\
0.09 \\
12.43 \\
11.78 \\
1.57 \\
0.03 \\
0.02 \\
0.03 \\
0.05 \\
100.71\end{array}$ \\
\hline $\begin{array}{l}\mathrm{LOI} \\
\mathrm{CO}_{2} \\
\mathrm{H}_{2} \mathrm{O} \\
\mathrm{FeOT}\end{array}$ & $\begin{array}{l}3.35 \\
0.24 \\
3.52 \\
4.62\end{array}$ & 9.70 & $\begin{array}{l}0.86 \\
0.06 \\
1.17 \\
5.53\end{array}$ & $\begin{array}{l}1.38 \\
4.68\end{array}$ & $\begin{array}{r}0.34 \\
0.04 \\
0.98 \\
11.85\end{array}$ & $\begin{array}{r}0.53 \\
13.80\end{array}$ & $\begin{array}{r}0.33 \\
0.08 \\
1.56 \\
16.71\end{array}$ & $\begin{array}{l}3.37 \\
0.02 \\
3.6 \\
4.71\end{array}$ \\
\hline \multicolumn{9}{|l|}{ Trace elements (ppm) } \\
\hline $\begin{array}{l}\mathrm{V} \\
\mathrm{Ti} \\
\mathrm{Cr} \\
\mathrm{Ni} \\
\mathrm{Sr} \\
\mathrm{Zr} \\
\mathrm{Ba} \\
\mathrm{Co} \\
\mathrm{Cu} \\
\mathrm{Zn} \\
\mathrm{Sc} \\
\mathrm{Y}\end{array}$ & $\begin{array}{r}27 \\
1619 \\
81 \\
298 \\
133 \\
70 \\
13 \\
32 \\
32 \\
31 \\
6.5 \\
8.8\end{array}$ & $\begin{array}{r}72 \\
4831 \\
147 \\
524 \\
131 \\
196 \\
11\end{array}$ & $\begin{array}{r}42 \\
1139 \\
513 \\
532 \\
127 \\
33 \\
14 \\
51 \\
52 \\
45 \\
11.4 \\
8.6\end{array}$ & $\begin{array}{r}34 \\
899 \\
109 \\
498 \\
151 \\
23 \\
18\end{array}$ & $\begin{array}{r}231 \\
12350 \\
28 \\
213 \\
170 \\
52 \\
0\end{array}$ & $\begin{array}{r}392 \\
15975 \\
555 \\
169 \\
129 \\
73 \\
14\end{array}$ & $\begin{array}{r}262 \\
30814 \\
140 \\
112 \\
141 \\
154 \\
16 \\
88 \\
86 \\
198 \\
15.4 \\
220.9\end{array}$ & $\begin{array}{r}65 \\
2278 \\
233 \\
376 \\
132 \\
35 \\
0\end{array}$ \\
\hline \multicolumn{9}{|l|}{ Rare earths (ppm) } \\
\hline $\begin{array}{l}\mathrm{La} \\
\mathrm{Ce} \\
\mathrm{Nd} \\
\mathrm{Sm} \\
\mathrm{Eu} \\
\mathrm{Gd} \\
\mathrm{Dy} \\
\mathrm{Er} \\
\mathrm{Yb} \\
\mathrm{Lu}\end{array}$ & $\begin{array}{l}1.99 \\
6.13 \\
3.40 \\
0.94 \\
0.40 \\
1.07 \\
1.23 \\
0.88 \\
0.94 \\
0.15\end{array}$ & $\begin{array}{r}3.16 \\
10.93 \\
10.13 \\
3.08 \\
1.18 \\
3.86 \\
4.25 \\
2.64 \\
2.35\end{array}$ & $\begin{array}{l}1.19 \\
4.16 \\
2.42 \\
0.81 \\
0.40 \\
1.12 \\
1.33 \\
0.85 \\
0.82 \\
0.12\end{array}$ & $\begin{array}{l}1.36 \\
4.44 \\
2.44 \\
0.68 \\
0.40 \\
0.78 \\
0.86 \\
0.61 \\
0.59 \\
0.08\end{array}$ & & $\begin{array}{r}5.68 \\
21.74 \\
24.24 \\
8.09 \\
2.38 \\
11.08 \\
11.94 \\
6.89 \\
5.28\end{array}$ & $\begin{array}{r}24.61 \\
91.20 \\
102.81 \\
31.10 \\
6.08 \\
38.66 \\
37.07 \\
19.82 \\
14.41 \\
2.19\end{array}$ & $\begin{array}{r}27.47 \\
100.32 \\
101.44 \\
31.81 \\
6.82 \\
41.00 \\
39.34 \\
22.06 \\
16.05\end{array}$ \\
\hline $\begin{array}{l}\text { Isotopes } \\
{ }^{87} \mathrm{Sr}{ }^{86} \mathrm{Sr}\end{array}$ & & & & & & & & \\
\hline
\end{tabular}

Notes: MOl-gabbro = olivine microgabbro. Other abbreviations are defined in the text and in Table 2. For isotopes, see Kempton and Casey (this volume).

during alteration, but the fact that the samples lie outside the weathering zone should help to minimize the effects compared to other abyssal peridotite suites. Previous attempts to use limited bulk-rock abyssal peridotite geochemical data in petrogenetic modeling utilized samples that were largely from the seafloor weathering zone (e.g., Falloon and Green, 1987). The Leg 153 residual harzburgites may be better suited in helping to establish bulk-rock petrogenetic trends in abyssal peridotites, although the primary geochemistry should not be regarded as unmodified.

\section{Downhole Variation in Major-Element Bulk-Rock Chemistry}

Three rock types characterized by distinct petrogeneses were sampled in Holes 920B and 920D from the ultramafic massif. Their petrogenesis is reflected in their bulk chemistry. These rock types include (1) residual peridotites, (2) more evolved cumulate ultramafic and mafic rocks that represent the crystallization or wallrock reaction products of channelized melts within the residual mantle, and (3) late stage, highly altered high-MgO diabase dikes that represent solidified mantle-derived melts. Although the data for diabases are presented in Tables 1 and 2 and various figures, they will be discussed more fully with other Leg 153 diabases below (also see Kempton and Casey, this volume).
The compositional range of coarse-grained rocks in Holes 920B and $920 \mathrm{D}$ is well depicted by the range of $\mathrm{Mg} \#$ plotted vs. depth (Fig. 8 ). The downhole variation for all but the most highly fractionated gabbroic samples $(\mathrm{Mg \# s}<65)$ in each hole is shown. The $\mathrm{Mg \# s} \mathrm{show}$ that the majority of samples, as expected, represent residual harzburgites with bulk Mg\#s in excess of 90 . Other samples represent or include the crystallization products of melts at various stages of fractionation (pyroxenites, wehrlites, gabbros, oxide gabbros) or approximate melt compositions (diabasic rocks). Some of the coarse cumulate rocks are highly fractionated oxide gabbros with bulk-rock

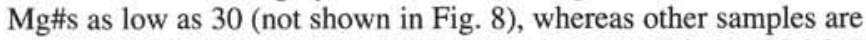
more primitive and approach residual mantle compositions with $\mathrm{Mg} \#$ $>90$. The range of values indicate that some of the ultramafic rocks (dunites, pyroxenites, and wehrlites) and gabbros represent or include the crystallization products of melts that are significantly fractionated with respect to melts that would be in equilibrium with residual mantle assemblages. The bulk-rock $\mathrm{Mg} \# \mathrm{~s}$ are much less than that typical of residual mantle ( $>90$ ) for these samples (e.g., Table 2).

Downhole variations in bulk-rock $\mathrm{Mg \#}$ for residual and intrusive ultramafic rocks also depict distinct depth intervals of lower $\mathrm{Mg \#} \mathrm{ul-}$ tramafic pyroxenites, wehrlites, dunites, websterite, and gabbroic rocks that occur in the stratigraphy and depart from the more monotonous and higher $\mathrm{Mg} \#$ values of the residual mantle. Both holes show 
Table 5. Bulk-rock major- and trace-element geochemistry of Site 923 mafic plutonic samples.

\begin{tabular}{|c|c|c|c|c|c|c|c|c|}
\hline Hole: & $923 \mathrm{~A}$ & $923 \mathrm{~A}$ & $923 \mathrm{~A}$ & $923 \mathrm{~A}$ & $923 \mathrm{~A}$ & $923 \mathrm{~A}$ & $923 \mathrm{~A}$ & $923 \mathrm{~A}$ \\
\hline Sample: & $2 \mathrm{R}-1,27-30$ & $2 R-1,70-75$ & $2 \mathrm{R}-1,108-113$ & 3R-1, 34-38 & $3 \mathrm{R}-1,126-132$ & $4 \mathrm{R}-1,3-10$ & $4 R-1,59-65$ & $4 \mathrm{R}-1,118-124$ \\
\hline Depth (mbsf): & 16.67 & 17.10 & 17.48 & 19.54 & 20.46 & 21.23 & 21.79 & 22.41 \\
\hline Laboratory (major elements): & ODP XRF & ODP XRF & ICP-AES-UH & ODP XRF & ICP-AES-UH & ICP-AES-UH & ICP-AES-UH & ICP-AES-UH \\
\hline Laboratory (trace and REEs): & ICP-AES-UH & ICP-AES-UH & ICP-AES-UH & ICP-AES-UH & ICP-AES-UH & ICP-AES-UH & ICP-AES-UH & ICP-AES-UH \\
\hline Lithology: & Metagabbro & Gabbro & Olivine gabbro & Olivine gabbro & Olivine gabbro & Oxide gabbro & Olivine gabbro & Gabbro \\
\hline \multicolumn{9}{|l|}{ Major elements (wt\%) } \\
\hline $\begin{array}{l}\mathrm{SiO}_{2} \\
\mathrm{TiO}_{2} \\
\mathrm{Al}_{2} \mathrm{O}_{3} \\
\mathrm{Fe}_{2} \mathrm{O}_{3} \\
\mathrm{MnO} \\
\mathrm{MgO} \\
\mathrm{CaO} \\
\mathrm{Na}_{2} \mathrm{O} \\
\mathrm{K}_{2} \mathrm{O} \\
\mathrm{P}_{2} \mathrm{O}_{5} \\
\mathrm{Cr}_{2} \mathrm{O}_{3} \\
\mathrm{NiO}^{\text {Total }}\end{array}$ & $\begin{array}{r}51.17 \\
0.32 \\
17.04 \\
6.97 \\
0.14 \\
9.93 \\
12.50 \\
2.41 \\
0.01 \\
0.00 \\
0.03 \\
0.02 \\
100.54\end{array}$ & $\begin{array}{r}52.49 \\
0.58 \\
13.74 \\
7.32 \\
0.16 \\
9.90 \\
14.13 \\
2.30 \\
0.03 \\
0.00 \\
0.02 \\
0.01 \\
100.67\end{array}$ & $\begin{array}{r}51.25 \\
0.42 \\
16.17 \\
6.05 \\
0.12 \\
8.17 \\
13.38 \\
2.99 \\
0.00 \\
0.01 \\
0.02 \\
0.01 \\
98.59\end{array}$ & $\begin{array}{r}51.79 \\
0.38 \\
16.52 \\
6.87 \\
0.14 \\
8.75 \\
12.82 \\
2.54 \\
0.00 \\
0.03 \\
0.01 \\
0.01 \\
99.86\end{array}$ & $\begin{array}{r}51.20 \\
0.54 \\
14.73 \\
7.60 \\
0.15 \\
9.86 \\
13.71 \\
2.61 \\
0.00 \\
0.05 \\
0.03 \\
0.01 \\
100.49\end{array}$ & $\begin{array}{r}46.54 \\
6.25 \\
16.58 \\
13.48 \\
0.18 \\
4.24 \\
9.33 \\
3.64 \\
0.09 \\
0.03 \\
0.01 \\
0.00 \\
100.38\end{array}$ & $\begin{array}{r}49.65 \\
0.49 \\
14.71 \\
9.20 \\
0.16 \\
10.17 \\
11.62 \\
2.50 \\
0.00 \\
0.06 \\
0.02 \\
0.01 \\
98.59\end{array}$ & $\begin{array}{r}51.66 \\
0.55 \\
15.29 \\
7.42 \\
0.15 \\
8.85 \\
12.99 \\
2.44 \\
0.03 \\
0.04 \\
0.02 \\
0.02 \\
99.46\end{array}$ \\
\hline $\begin{array}{l}\mathrm{LOI} \\
\mathrm{CO}_{2} \\
\mathrm{H}_{2} \mathrm{O} \\
\mathrm{FeOT}\end{array}$ & $\begin{array}{l}0.71 \\
0.02 \\
1.02 \\
6.27\end{array}$ & $\begin{array}{l}0.27 \\
0.05 \\
0.66 \\
6.59\end{array}$ & 0.67 & $\begin{array}{r}-0.01 \\
0.05 \\
0.45 \\
6.18\end{array}$ & 0.25 & 0.45 & 8.28 & $\begin{array}{r}-0.09 \\
0.04 \\
0.34 \\
6.68\end{array}$ \\
\hline $\begin{array}{l}\text { Trace elements (ppm) } \\
\text { V } \\
\mathrm{Ti} \\
\mathrm{Cr} \\
\mathrm{Ni} \\
\mathrm{Sr} \\
\mathrm{Zr} \\
\mathrm{Ba} \\
\mathrm{Co} \\
\mathrm{Cu} \\
\mathrm{Zn} \\
\mathrm{Sc} \\
\mathrm{Y}\end{array}$ & $\begin{array}{r}143 \\
1918 \\
227 \\
169 \\
157 \\
14 \\
10 \\
51 \\
119 \\
38 \\
36.6 \\
8.7\end{array}$ & $\begin{array}{r}235 \\
3477 \\
115 \\
59 \\
111 \\
63 \\
9 \\
40 \\
40 \\
42 \\
56.9 \\
23.7\end{array}$ & $\begin{array}{r}182 \\
2518 \\
121 \\
82 \\
143 \\
27 \\
14\end{array}$ & $\begin{array}{r}150 \\
2278 \\
64 \\
89 \\
146 \\
14 \\
9 \\
51 \\
116 \\
29 \\
40.3 \\
10.8\end{array}$ & $\begin{array}{r}200 \\
3237 \\
199 \\
110 \\
129 \\
54 \\
14\end{array}$ & $\begin{array}{r}677 \\
37459 \\
81 \\
10 \\
174 \\
83 \\
10\end{array}$ & $\begin{array}{r}173 \\
2938 \\
119 \\
117 \\
127 \\
56 \\
13\end{array}$ & $\begin{array}{r}188 \\
3297 \\
130 \\
155 \\
128 \\
65 \\
11 \\
40 \\
77 \\
41 \\
47.3 \\
22.8\end{array}$ \\
\hline $\begin{array}{l}\text { Rare earths (ppm) } \\
\text { La } \\
\text { Ce } \\
\text { Nd } \\
\text { Sm } \\
\text { Eu } \\
\text { Gd } \\
\text { Dy } \\
\text { Er } \\
\text { Yb } \\
\text { Lu }\end{array}$ & $\begin{array}{l}1.07 \\
2.40 \\
1.19 \\
0.54 \\
0.44 \\
1.03 \\
1.36 \\
0.87 \\
0.82 \\
0.13\end{array}$ & $\begin{array}{l}1.80 \\
6.65 \\
5.70 \\
2.02 \\
0.75 \\
2.94 \\
3.75 \\
2.41 \\
2.23 \\
0.35\end{array}$ & $\begin{array}{l}0.67 \\
3.17 \\
2.67 \\
0.95 \\
0.57 \\
1.40 \\
2.03 \\
1.38 \\
1.20 \\
0.18\end{array}$ & $\begin{array}{l}0.75 \\
2.52 \\
1.75 \\
0.75 \\
0.51 \\
1.32 \\
1.76 \\
1.13 \\
1.00 \\
0.16\end{array}$ & $\begin{array}{l}1.75 \\
6.72 \\
5.39 \\
1.97 \\
0.80 \\
2.96 \\
3.54 \\
2.22 \\
2.10 \\
0.32\end{array}$ & $\begin{array}{l}0.88 \\
3.24 \\
3.52 \\
1.36 \\
1.07 \\
2.16 \\
2.74 \\
1.75 \\
1.65\end{array}$ & $\begin{array}{l}1.68 \\
6.38 \\
5.20 \\
1.82 \\
0.84 \\
2.63 \\
3.06 \\
1.92 \\
1.84 \\
0.28\end{array}$ & $\begin{array}{l}2.23 \\
7.50 \\
6.41 \\
2.15 \\
0.78 \\
2.96 \\
3.61 \\
2.30 \\
2.13 \\
0.32\end{array}$ \\
\hline \multicolumn{9}{|l|}{ Isotopes } \\
\hline
\end{tabular}

increased $\mathrm{CaO}$ content and elevated clinopyroxene occurs in intervals between $\sim 155$ and 160 mbsf and between 190 and 200 mbsf. It is not surprising that the downhole chemistry shows similar variations at a given depth because Holes were drilled only $20 \mathrm{~m}$ apart. The downhole variations reflect sympathetic changes in pyroxene/ olivine ratios and variation in the percent of clinopyroxene, which are commonly associated with changes in $\mathrm{Mg \# s}$. The sympathetic variation in both holes appears to indicate discrete zones of melt impregnation, pyroxene addition, and crystal fractionation of melt within the mantle sections. The high- $\mathrm{CaO}$ zones also tend to be the intervals where gabbroic and pyroxenite veins (e.g., Samples 153-920D-12R3, Pieces 5-6; 153-920D-13R-1, Pieces 8-9) have also been recorded (Shipboard Scientific Party, 1995).

\section{Estimates of Extent of Melting and Mantle Refertilization}

A plot of $\mathrm{MgO} / \mathrm{SiO}_{2}$ and $\mathrm{Al}_{2} \mathrm{O}_{3} / \mathrm{SiO}_{2}$ ratios for MARK residual harzburgites, wehrlites, and pyroxenites from Hole 920B and 920B (Fig. 11) depicts the extent of pyroxene depletion for the residual mantle samples compared with available Mid-Atlantic Ridge abyssal peridotite bulk-rock analyses (J.F. Casey and S. Silantyev, unpubl. data). $\mathrm{MgO} / \mathrm{SiO}_{2}$ increases with increasing modal olivine and $\mathrm{Al}_{2} \mathrm{O}_{3} /$ $\mathrm{SiO}_{2}$ decreases with decreasing pyroxene content. Also shown for comparison are residual mantle samples from the BOIC (J.F. Casey, unpubl. data) and proposed upper mantle compositions including Primitive Upper Mantle (PUM) (Sun and McDonough, 1989), Tinaquillo Lherzolite (TQ), MPY-90, and Hawaiian Pyrolite (Falloon and Green, 1987). The BOIC residual mantle array was chosen for comparison because it shows a particularly broad range of composition and near linear depletion trend from more fertile lherzolite to depleted harzburgite. In addition, alteration appears to have been largely isochemical in the BOIC mantle sections, except for addition of volatiles (Komor et al., 1985b). The MARK samples plot along the same trend as the BOIC samples, but plot higher in $\mathrm{MgO} / \mathrm{SiO}_{2}$ than most abyssal peridotites that are sampled from the seafloor weathering zone where $\mathrm{MgO}$ loss is common. The MARK peridotites plot at the $\mathrm{Al}_{2} \mathrm{O}_{3} / \mathrm{SiO}_{2}$ rich side of the abyssal peridotite array and BOIC dense array, indicating that the MARK peridotites are among the least depleted in $\mathrm{Al}_{2} \mathrm{O}_{3}$ (and total pyroxene) of abyssal peridotites analyzed to date. This is consistent with the low $\mathrm{Cr} \#$ of spinels and high $\mathrm{Al}_{2} \mathrm{O}_{3}$ content of pyroxene within these abyssal peridotites, as previously discussed. Compared to various proposed mantle sources shown, however, they are significantly more depleted in pyroxene (i.e., $\mathrm{Al}_{2} \mathrm{O}_{3} / \mathrm{SiO}_{2}$ ). The choice of starting compositions ultimately determines the extent of melting calculated. TQ, MPY-90, and HPY are possible sources; TQ and HPY are somewhat more depleted than 
Table 5 (continued).

\begin{tabular}{|c|c|c|c|c|c|c|c|c|}
\hline Hole: & $923 \mathrm{~A}$ & $923 \mathrm{~A}$ & $923 \mathrm{~A}$ & $923 \mathrm{~A}$ & $923 \mathrm{~A}$ & $923 \mathrm{~A}$ & $923 \mathrm{~A}$ & $923 \mathrm{~A}$ \\
\hline Sample: & $5 R-1,78-84$ & $5 R-2,109-11$ & $6 \mathrm{R}-1,22-25$ & $6 \mathrm{R}-1,42-49$ & $7 R-1,73-79$ & $7 R-2,1-7$ & $8 \mathrm{R}-1,29-32$ & $8 R-1,48-52$ \\
\hline Depth (mbsf): & 23.78 & 25.00 & 24.92 & 25.12 & 27.63 & 28.32 & 29.29 & 29.48 \\
\hline Laboratory (major elements): & ICP-AES-UH & ODP XRF & ODP XRF & ICP-AES-UH & ICP-AES-UH & ODP XRF & ODP XRF & ICP-AES-UH \\
\hline Laboratory (trace and REEs): & ICP-AES-UH & ICP-AES-UH & ICP-AES-UH & ICP-AES-UH & ICP-AES-UH & ICP-AES-UH & ICP-AES-UH & ICP-AES-UH \\
\hline Lithology: & Olivine gabbro & Olivine gabbro & Olivine gabbro & Olivine gabbro & Olivine gabbro & Olivine gabbro & Olivine gabbro & Gabbro \\
\hline \multicolumn{9}{|l|}{ Major elements (wt\%) } \\
\hline $\mathrm{SiO}_{2}$ & 50.39 & 51.79 & 50.92 & 50.16 & 50.68 & 48.49 & 50.21 & 51.32 \\
\hline $\mathrm{TiO}_{2}$ & 0.36 & 0.44 & 0.35 & 0.35 & 0.32 & 0.63 & 0.36 & 0.41 \\
\hline $\begin{array}{l}\mathrm{Al}_{2} \mathrm{O}_{3} \\
\mathrm{Fe}_{2} \mathrm{O}_{3}\end{array}$ & 15.39 & 16.16 & 16.55 & 15.60 & 15.76 & 21.56 & 23.19 & 17.53 \\
\hline $\begin{array}{l}\mathrm{Fe}_{2} \mathrm{O}_{3} \\
\mathrm{MnO}\end{array}$ & 6.81 & 6.18 & 6.96 & 6.81 & 6.29 & 4.80 & 4.91 & 4.76 \\
\hline $\begin{array}{l}\mathrm{MnO} \\
\mathrm{MgO}\end{array}$ & 0.13 & 0.13 & 0.13 & 0.13 & 0.12 & 0.09 & 0.08 & 0.10 \\
\hline $\begin{array}{l}\mathrm{MgO} \\
\mathrm{CaO}\end{array}$ & 9.72 & 9.23 & 9.87 & 9.83 & 10.32 & 8.11 & 5.69 & 7.21 \\
\hline $\begin{array}{l}\mathrm{CaO} \\
\mathrm{Na}_{2} \mathrm{O}\end{array}$ & 13.24 & 13.77 & 12.98 & 13.62 & 13.67 & 13.33 & 12.12 & 14.11 \\
\hline $\begin{array}{l}\mathrm{Na}_{2} \mathrm{O} \\
\mathrm{K}_{2} \mathrm{O}\end{array}$ & 2.46 & 2.40 & 2.32 & 2.41 & 2.19 & 2.11 & 2.88 & 2.84 \\
\hline $\mathrm{K}_{2} \mathrm{O}$ & 0.00 & 0.01 & 0.01 & 0.00 & 0.00 & 0.04 & 0.03 & 0.03 \\
\hline $\mathrm{P}_{2} \mathrm{O}_{5}$ & 0.00 & 0.00 & 0.00 & 0.02 & 0.00 & 0.12 & 0.02 & 0.03 \\
\hline $\mathrm{Cr}_{2} \mathrm{O}_{3}$ & 0.02 & 0.02 & 0.03 & 0.04 & 0.06 & 0.06 & 0.01 & 0.04 \\
\hline $\mathrm{NiO}$ & 0.01 & 0.01 & 0.02 & 0.01 & 0.02 & 0.03 & 0.02 & 0.01 \\
\hline Total & 98.53 & 100.14 & 100.13 & 98.98 & 99.42 & 99.37 & 99.52 & 98.39 \\
\hline LOI & 0.26 & -0.02 & 0.20 & 0.43 & 0.40 & 0.61 & 0.75 & 0.52 \\
\hline $\mathrm{CO}_{2}$ & & 0.01 & 0.04 & & & 0.04 & 0.05 & \\
\hline $\begin{array}{l}\mathrm{H}_{2} \mathrm{O} \\
\mathrm{FeOT}\end{array}$ & 6,3 & 0.33 & 0.28 & & & 0.72 & 0.58 & \\
\hline $\mathrm{FeOT}$ & 6.13 & 5.56 & 6.26 & 6.13 & 5.66 & 4.32 & 4.42 & 4.28 \\
\hline \multicolumn{9}{|l|}{ Trace elements (ppm) } \\
\hline V & 159 & 198 & 146 & 160 & 131 & 112 & 51 & 166 \\
\hline $\mathrm{Ti}$ & 2158 & 2638 & 2098 & 2098 & 1918 & 3777 & 2158 & 2432 \\
\hline $\mathrm{Cr}$ & 116 & 119 & 177 & 246 & 398 & 423 & 42 & 263 \\
\hline $\mathrm{Ni}$ & 113 & 98 & 123 & 113 & 127 & 216 & 152 & 108 \\
\hline $\mathrm{Sr}$ & 123 & 136 & 135 & 124 & 101 & 140 & 185 & 148 \\
\hline $\mathrm{Zr}$ & 19 & 20 & 13 & 19 & 13 & 66 & 24 & 35 \\
\hline $\mathrm{Ba}$ & 9 & 9 & 9 & 8 & 3 & 11 & 12 & 9 \\
\hline Co & & 37 & 46 & & & 34 & 35 & \\
\hline $\mathrm{Cu}$ & & 68 & 101 & & & 48 & 81 & \\
\hline $\mathrm{Zn}$ & & 26 & 33 & & & 27 & 30 & \\
\hline $\mathrm{Sc}$ & 44.3 & 44.5 & 38.6 & 43.9 & 42.3 & 18.3 & 11.4 & 41.4 \\
\hline Y & 12.1 & 12.3 & 9.9 & 11.4 & 10.5 & 22.7 & 10.1 & 15.7 \\
\hline \multicolumn{9}{|l|}{ Rare earths (ppm) } \\
\hline $\mathrm{La}$ & 0.57 & 0.89 & 0.81 & 0.43 & 0.78 & 3.06 & 1.40 & 0.90 \\
\hline $\mathrm{Ce}$ & 2.57 & 2.71 & 2.46 & 2.31 & 3.35 & 8.40 & 4.80 & 3.66 \\
\hline $\mathrm{Nd}$ & 2.23 & 1.92 & 1.47 & 1.88 & 2.00 & 7.33 & 3.12 & 3.56 \\
\hline $\mathrm{Sm}$ & 0.85 & 0.81 & 0.66 & 0.76 & 0.79 & 2.40 & 1.05 & 1.31 \\
\hline $\mathrm{Eu}$ & 0.55 & 0.56 & 0.50 & 0.53 & 0.39 & 0.84 & 0.72 & 0.70 \\
\hline $\mathrm{Gd}$ & 1.33 & 1.41 & 1.20 & 1.28 & 1.27 & 3.22 & 1.45 & 1.97 \\
\hline Dy & 1.85 & 1.91 & 1.59 & 1.76 & 1.57 & 3.61 & 1.65 & 2.57 \\
\hline Er & 1.21 & 1.21 & 1.02 & 1.17 & 1.03 & 2.24 & 0.98 & 1.58 \\
\hline $\mathrm{Yb}$ & 1.06 & 1.12 & 0.92 & 1.01 & 1.00 & 2.01 & 0.94 & 1.37 \\
\hline Lu & 0.15 & 0.16 & 0.14 & 0.15 & 0.13 & 0.32 & 0.13 & \\
\hline $\begin{array}{l}\text { Isotopes } \\
{ }^{87} \mathrm{Sr} /{ }^{86} \mathrm{Sr}\end{array}$ & & & & & & & & \\
\hline
\end{tabular}

MPY-90 and would yield lower extents of melting. Also shown are various related compositions used in melting experiments. MPY-90 is considered a likely fertile MORB mantle source (Falloon and Green, 1987; Niu and Batiza, 1991).

Pearce and Parkinson (1993) have proposed a method of evaluating melting and depletion of the mantle utilizing major and trace elements ordered from compatible to highly incompatible elements with respect to melting of a spinel lherzolite. They have proposed a fertile MORB mantle (FMM) composition for normalization. FMM is an estimate of the average global convecting upper mantle reservoir from which N-MORB is generated. Its composition is derived from published whole-rock data from orogenic lherzolite and nodules that show no evidence of metasomatic enrichment and are nearly identical to MPY-90 (Falloon and Green, 1987), which was used in the modeling of Niu and Batiza (1991) presented above. Computed compositions of residual mantle normalized to FMM after 5\%, 10\%, $15 \%$, $20 \%$, and $25 \%$ melting are based on regression of element concentration vs. percentage of modal clinopyroxene in natural peridotite samples. Pearce and Parkinson (1993) assume that clinopyroxene is exhausted after $25 \%$ melting, which is supported by experimental data. The mantle depletion patterns predicted by the melting models of
Pearce and Parkinson (1993) are shown in Figure 12A. Increasing extents of melting lead to systematic and gradual increases in the most compatible elements within the mantle and to substantial decreases in the more highly incompatible elements (e.g., Johnson et al., 1990).

Spidergrams of certain of our major- and trace-element data normalized to FMM show the characteristics of MARK residual peridotites, as well as characteristics of pyroxenites, wehrlites, lherzolite, gabbros, and diabases veins and dikes that have intruded these residual peridotites. Elements are listed from right to left in order of their increasing compatibility during mantle melting. Figures $12 \mathrm{~B}-12 \mathrm{~F}$ depict typical examples of trace- and major-element patterns for residual peridotites from Hole 920D, as well as more enriched ultramafic, gabbroic, and diabasic intrusive rocks sampled from the same cores. For harzburgite, these patterns appear to indicate an enrichment in $\mathrm{Zr}$, the most incompatible element plotted, and an overall $\mathrm{U}$ shaped pattern not unlike the patterns observed in residual mantle of ophiolites (Frey, 1984). Similar enrichments in highly incompatible light REEs (LREE) and U-shaped REE patterns are observed in other Leg 153 residual harzburgites (Stephens, this volume). These results may suggest that there was a late enrichment of highly incompatible elements within many MARK residual peridotites during melt trans- 
Table 5 (continued).

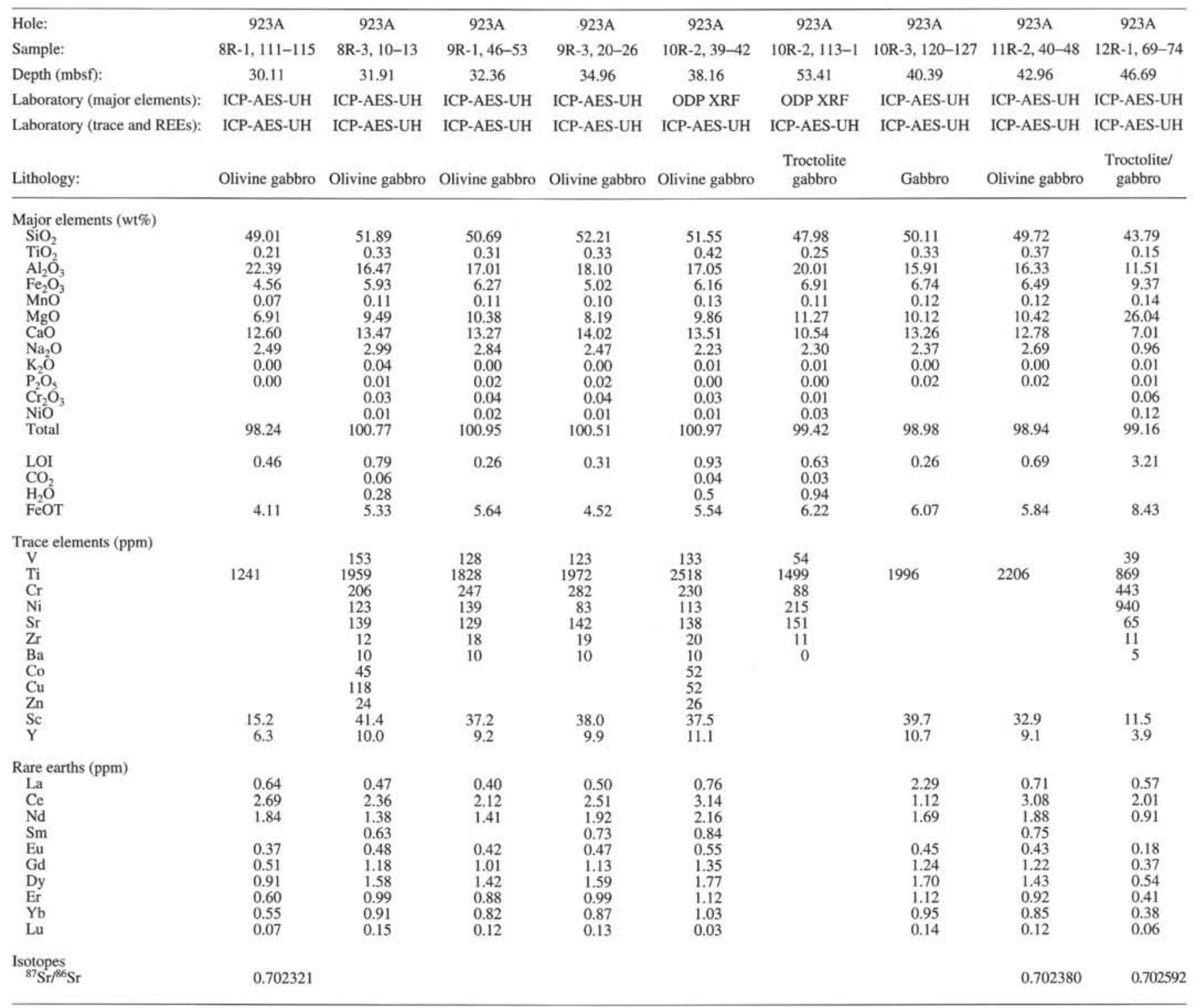

port by porous flow. These melts may have been similar in composition to melts that solidified during channelized flow to form intrusive ultramafic cumulate veins (e.g., wehrlites, pyroxenites, and gabbros). These melts appear to have been especially enriched in these highly incompatible elements.

In addition, the spidergrams indicate significant variability in $\mathrm{CaO}$ in both XRF and ICP data that does not result from analytical precision. This indicates that some of the harzburgites tend to be variably depleted in $\mathrm{CaO}$, with respect to adjacent elements in the compatibility scheme. $\mathrm{CaO}$ abundances should be largely controlled by the clinopyroxene abundances because clinopyroxene is the only calcic phase in the primary mode of the residual peridotites (i.e., unless the depletion is caused by alteration effects; see discussion above). If primary, $\mathrm{CaO}$ and thus clinopyroxene tend to be more depleted than predicted by the melting model in many samples.

Ultramafic and mafic intrusive rocks (pyroxenites, wehrlites, etc.) tend to be enriched in highly incompatible elements and depleted in compatible elements relative to FMM and lack the U-shaped patterns characteristic of residual peridotites. Several of the pyroxenitic intrusive rocks show prominent negative $\mathrm{Ti}$ anomalies that may be related to extensive $\mathrm{Fe}$-Ti oxide crystal fractionation before intrusion and/or reaction with the wallrock peridotite. Oxide pyroxenites were recovered from both peridotite holes (Shipboard Scientific Party, 1995).

Figures 13 and 14 display the melting trends defined by Pearce and Parkinson (1993) for various major and trace elements. $\mathrm{Al}_{2} \mathrm{O}_{3}$ tends to be one of the most immobile major-element oxides during alteration. The $\mathrm{MgO} / \mathrm{FMM}$ vs. $\mathrm{Al}_{2} \mathrm{O}_{3} / \mathrm{FMM}$ diagram shows that MARK residual peridotites generally plot on the melting trend in the range from $15 \%$ to $20 \%$ partial melting. This presumably represents the maximum extent of melting Fmax in the polybaric residual melting column. Initiating melting within the spinel-lherzolite facies at 20 kbar and assuming that $F \max =15 \%-20 \%$ and that $1.2 \mathrm{wt} \%$ of partial melting per kbar (Klein and Langmuir, 1987), the cessation of melting is likely to have occurred at $\sim 3-7 \mathrm{kbar}$. Thus, the base of the mechanical lithosphere may be as shallow as $\sim 9-10 \mathrm{~km}$ depth below the seafloor in the MARK region, consistent with the results of Toomey et al. (1988). Based on the value of Fmax of $15 \%-20 \%$, the average value of melting within the melting column (Plank and Langmuir, 1992; Plank et al., 1995) is likely to have been $~ 8 \%-11 \%$ using a near-fractional melting model. Intrusive ultramafic and gabbroic 
Table 5 (continued).

\begin{tabular}{|c|c|c|c|c|c|c|c|}
\hline Hole: & $923 \mathrm{~A}$ & $923 \mathrm{~A}$ & $923 \mathrm{~A}$ & $923 \mathrm{~A}$ & $923 \mathrm{~A}$ & $923 \mathrm{~A}$ & $923 \mathrm{~A}$ \\
\hline Sample: & $13 R-1,48-55$ & $14 \mathrm{R}-1,116-123$ & $14 \mathrm{R}-1,116-123$ & $15 R-1,93-96$ & $15 R-2,0-6$ & $15 R-3,4-12$ & $16 \mathrm{R}-1,39-45$ \\
\hline Depth (mbsf): & 51.28 & 56.66 & 56.66 & 61.23 & 61.55 & 62.91 & 65.09 \\
\hline Laboratory (major elements): & ICP-AES-UH & ICP-AES-UH & ICP-AES-UH & ODP XRFICP-AES-UH & ICP-AES-UH & ICP-AES-UH & ICP-AES-UH \\
\hline Laboratory (trace and REEs): & ICP-AES-UH & ICP-AES-UH & ICP-AES-UH & ICP-AES-UH & ICP-AES-UH & ICP-AES-UH & ICP-AES-UH \\
\hline Lithology: & Gabbro & Gabbro & & Olivine gabbro & Gabbro & Olivine gabbro & Gabbro \\
\hline $\begin{array}{l}\text { Major elements (wt\%) } \\
\mathrm{SiO}_{2} \\
\mathrm{TiO}_{2} \\
\mathrm{Al}_{2} \mathrm{O}_{3} \\
\mathrm{Fe}_{2} \mathrm{O}_{3} \\
\mathrm{MnO} \\
\mathrm{MgO} \\
\mathrm{CaO} \\
\mathrm{Na}_{2} \mathrm{O} \\
\mathrm{K}_{2} \mathrm{O} \\
\mathrm{P}_{2} \mathrm{O}_{5} \\
\mathrm{Cr}_{2} \mathrm{O}_{3} \\
\mathrm{NiO} \\
\text { Total }\end{array}$ & $\begin{array}{r}50.49 \\
0.30 \\
18.16 \\
6.14 \\
0.11 \\
8.61 \\
12.63 \\
2.64 \\
0.00 \\
0.01 \\
0.12 \\
0.02 \\
99.23\end{array}$ & $\begin{array}{r}49.48 \\
0.33 \\
17.80 \\
6.62 \\
0.11 \\
10.39 \\
12.73 \\
2.61 \\
0.00 \\
0.01 \\
0.08 \\
0.03 \\
100.18\end{array}$ & $\begin{array}{r}49.48 \\
0.33 \\
17.80 \\
6.62 \\
0.11 \\
10.39 \\
12.73 \\
2.61 \\
0.00 \\
0.01 \\
0.08 \\
0.03 \\
100.18\end{array}$ & $\begin{array}{r}47.85 \\
0.58 \\
21.39 \\
6.49 \\
0.11 \\
10.76 \\
10.60 \\
2.27 \\
0.03 \\
0.05 \\
0.00 \\
0.04 \\
100.17\end{array}$ & $\begin{array}{r}50.64 \\
0.48 \\
18.93 \\
7.35 \\
0.12 \\
7.82 \\
11.24 \\
3.10 \\
0.06 \\
0.09 \\
0.04 \\
0.02 \\
99.86\end{array}$ & $\begin{array}{r}48.60 \\
0.16 \\
22.48 \\
4.94 \\
0.08 \\
9.46 \\
12.12 \\
2.24 \\
0.01 \\
0.00 \\
0.05 \\
0.03 \\
100.17\end{array}$ & $\begin{array}{r}48.66 \\
0.24 \\
21.75 \\
4.28 \\
0.07 \\
8.55 \\
13.52 \\
2.46 \\
0.00 \\
0.02 \\
0.06 \\
0.02 \\
99.64\end{array}$ \\
\hline $\begin{array}{l}\mathrm{LOI} \\
\mathrm{CO}_{2} \\
\mathrm{H}_{2} \mathrm{O} \\
\mathrm{FeOT}\end{array}$ & 0.31 & 1.00 & 1.00 & $\begin{array}{l}0.88 \\
0.06 \\
0.96 \\
5.84\end{array}$ & 0.97 & $\begin{array}{l}0.63 \\
0.03 \\
0.7 \\
4.45\end{array}$ & 0.64 \\
\hline $\begin{array}{l}\text { Trace elements (ppm) } \\
\text { V } \\
\mathrm{Ti} \\
\mathrm{Cr} \\
\mathrm{Ni} \\
\mathrm{Sr} \\
\mathrm{Zr} \\
\mathrm{Ba} \\
\mathrm{Co} \\
\mathrm{Cu} \\
\mathrm{Zn} \\
\mathrm{Sc} \\
\mathrm{Y}\end{array}$ & $\begin{array}{r}107 \\
1793 \\
833 \\
129 \\
127 \\
17 \\
6\end{array}$ & $\begin{array}{r}127 \\
2002 \\
561 \\
200 \\
116 \\
16 \\
8\end{array}$ & $\begin{array}{r}127 \\
2002 \\
561 \\
200 \\
116 \\
16 \\
8\end{array}$ & $\begin{array}{r}68 \\
3477 \\
17 \\
278 \\
145 \\
52 \\
12 \\
54 \\
72 \\
43 \\
8.9 \\
10.1\end{array}$ & $\begin{array}{r}118 \\
2891 \\
275 \\
125 \\
143 \\
122 \\
10\end{array}$ & $\begin{array}{c}33 \\
959 \\
324 \\
228 \\
153 \\
15 \\
12 \\
35 \\
103 \\
21 \\
10.4 \\
3.2\end{array}$ & $\begin{array}{r}57 \\
1415 \\
430 \\
177 \\
128 \\
16 \\
5\end{array}$ \\
\hline $\begin{array}{l}\text { Rare earths (ppm) } \\
\mathrm{La} \\
\mathrm{Ce} \\
\mathrm{Nd} \\
\mathrm{Sm} \\
\mathrm{Eu} \\
\mathrm{Gd} \\
\mathrm{Dy} \\
\mathrm{Er} \\
\mathrm{Yb} \\
\mathrm{Lu}\end{array}$ & $\begin{array}{l}0.71 \\
3.08 \\
1.88 \\
0.75 \\
0.43 \\
1.22 \\
1.43 \\
0.92 \\
0.85 \\
0.12\end{array}$ & $\begin{array}{l}0.69 \\
3.21 \\
1.80 \\
0.65 \\
0.44 \\
0.97 \\
1.22 \\
0.75 \\
0.80 \\
0.07\end{array}$ & $\begin{array}{l}0.69 \\
3.21 \\
1.80 \\
0.65 \\
0.44 \\
0.97 \\
1.22 \\
0.75 \\
0.80 \\
0.07\end{array}$ & $\begin{array}{l}2.00 \\
5.71 \\
4.11 \\
1.23 \\
0.64 \\
1.52 \\
1.64 \\
1.04 \\
0.95 \\
0.17\end{array}$ & $\begin{array}{l}2.94 \\
9.63 \\
7.93 \\
2.42 \\
0.90 \\
3.05 \\
3.64 \\
2.38 \\
2.12\end{array}$ & $\begin{array}{l}0.74 \\
2.30 \\
1.10 \\
0.36 \\
0.36 \\
0.49 \\
0.55 \\
0.36 \\
0.32 \\
0.05\end{array}$ & $\begin{array}{l}0.81 \\
3.14 \\
1.76 \\
0.63 \\
0.46 \\
0.92 \\
0.99 \\
0.60 \\
0.60 \\
0.08\end{array}$ \\
\hline $\begin{array}{l}\text { Isotopes } \\
{ }^{87} \mathrm{Sr} /{ }^{86} \mathrm{Sr}\end{array}$ & & & & & & & \\
\hline
\end{tabular}

samples recovered from these holes plot in compositional fields outside that expected to represent the residuum of partial melting because they represent the result of the addition of melt crystallization products to the residuum sometime after melting. Magmatically twinned clinopyroxene within these dikes and veins and within the residual mantle adjacent to these veins attest to the cumulate nature the pyroxenites and the local impregnation and refertilization of the adjacent residual mantle. The extent of melting would be limited to $10 \%-15 \%$ if a more depleted source such as Tinaquillo Lherzolite is chosen, but this seems unlikely for the MARK region.

The more incompatible trace elements such as $\mathrm{Zr}$, $\mathrm{Ti}$, and $\mathrm{V}$ when plotted vs. $\mathrm{Al}_{2} \mathrm{O}_{3} / \mathrm{FMM}$, plot slightly to significantly off the melting trend. They are generally more enriched than predicted by the melting model. $\mathrm{Zr}$, the most incompatible element plotted, shows a large departure from the melting trend, although the fact that values are proximal to detection limits for both the XRF and ICP limits the precision of these analyses. These enrichments, however, appear to result in U-shaped patterns on the spidergram, not dissimilar to the Ushaped REE patterns noted for dunites and harzburgites within depleted ophiolites (Frey, 1984; McDonough and Frey, 1989). Such patterns are generally interpreted to result from a postmelting metasomatic enrichments caused by a melt or fluid phase.

In an oceanic ridge setting with a polybaric melting column, these bulk-rock patterns could result from mixing of a depleted residue of melting with melts derived from deeper in the column. The melts should be more enriched incompatible elements if they are generated deeper in the mantle melting column at lower extents of melting (e.g., Song and Frey, 1989). These melts may represent early LREE and incompatible-element-enriched, near-fractional melts generated near the mantle solidus at significant depths in the melting column or their fractionally crystallized derivatives. The history of these enriched melts could be complex if they underwent mixing and fractionation effects on the way to shallower levels. Direct evidence for the enriched and fractionated nature of these impregnating melts has been observed in the geochemistry of ultramafic and mafic intrusive rocks with Holes 920B and 920D.

\section{Crystallization Order and Composition of Melts Involved in Late-Stage Veining Within the Residual Mantle}

If melt migration of early to late-formed melts in the mantle is channelized, its migration may be slow enough to reequilibrate with mantle phases (i.e., $\mathrm{Fe} / \mathrm{Mg}$ exchange) at lower pressure because of high diffusion rates for these elements between melt and olivine (Hess, 1992). However, its migration may be fast enough to retain the trace-element signature of partial melts or fractionated melts formed deeper in the melting column or lithosphere (e.g., see Freer, 1981). Many of these channels would appear to be in the form of veins ob- 
Table 5 (continued).

\begin{tabular}{|c|c|c|c|c|c|c|c|}
\hline Hole: & $923 \mathrm{~A}$ & $923 \mathrm{~A}$ & $923 \mathrm{~A}$ & $923 \mathrm{~A}$ & $923 \mathrm{~A}$ & $923 \mathrm{~A}$ & $923 \mathrm{~A}$ \\
\hline Sample: & $16 \mathrm{R}-1,119-126$ & $16 \mathrm{R}-2,77-83$ & $16 \mathrm{R}-3,23-28$ & $16 R-3,74-80$ & $16 \mathrm{R}-3,101-107$ & $16 \mathrm{R}-3,118-124$ & $16 \mathrm{R}-4,60-66$ \\
\hline Depth (mbsf) & 65.89 & 66.73 & 67.36 & 67.90 & 68.14 & 68.31 & 69.01 \\
\hline Laboratory (major elements): & ICP-AES-UH & ICP-AES-UH & ODP XRF & ICP-AES-UH & & ICP-AES - UH & ODP XRF \\
\hline Laboratory (trace and REEs): & ICP-AES-UH & ICP-AES-UH & ICP-AES-UH & ICP-AES-UH & ICP-AES - UH & ICP-AES - UH & ICP-AES - UH \\
\hline Lithology: & Olivine gabbro & Olivine gabbro & Troctolite/gabbro & Olivine gabbro & & Gabbro & Olivine gabbro \\
\hline \multicolumn{8}{|l|}{ Major elements (wt\%) } \\
\hline $\mathrm{SiO}_{2}$ & 48.86 & 48.96 & 48.66 & 49.59 & 50.90 & 51.58 & 50.21 \\
\hline $\mathrm{TiO}_{2}$ & 0.23 & 0.27 & 0.30 & 0.27 & 0.47 & 0.59 & 0.35 \\
\hline $\mathrm{Al}_{2} \mathrm{O}_{3}$ & 20.97 & 21.00 & 20.06 & 20.71 & 15.59 & 16.15 & 15.85 \\
\hline $\mathrm{Fe}_{2} \mathrm{O}_{3}$ & 5.11 & 5.21 & 4.80 & 4.99 & 6.80 & 7.30 & 7.45 \\
\hline $\mathrm{MnO}$ & 0.08 & 0.09 & 0.08 & 0.09 & 0.13 & 0.15 & 0.13 \\
\hline $\mathrm{MgO}$ & 11.18 & 10.16 & 10.11 & 9.59 & 9.72 & 8.10 & 11.08 \\
\hline $\mathrm{CaO}$ & 12.41 & 12.91 & 12.97 & 12.94 & 13.88 & 12.47 & 12.42 \\
\hline $\mathrm{Na}_{2} \mathrm{O}$ & 2.15 & 2.62 & 2.63 & 2.09 & 2.46 & 3.13 & 2.10 \\
\hline $\mathrm{K}_{2} \mathrm{O}$ & 0.00 & 0.02 & 0.01 & 0.01 & 0.00 & 0.05 & 0.02 \\
\hline $\mathrm{P}_{2} \mathrm{O}_{5}$ & 0.01 & 0.02 & 0.04 & 0.00 & 0.00 & 0.03 & 0.00 \\
\hline $\mathrm{Cr}_{2} \mathrm{O}_{3}$ & 0.09 & 0.05 & 0.16 & 0.08 & 0.02 & 30.0 & 10.05 \\
\hline $\mathrm{NiO}$ & 0.03 & 0.03 & 0.03 & 0.03 & 0.01 & 0.02 & 0.02 \\
\hline Total & 101.12 & 101.33 & 99.84 & 100.39 & 99.98 & 99.57 & 99.68 \\
\hline LOI & 1.16 & 1.28 & & 0.69 & 0.79 & 1.66 & 0.88 \\
\hline $\mathrm{CO}_{2}$ & & & & 0.07 & & 0.05 & \\
\hline $\mathrm{H}_{2} \mathrm{O}$ & & & & 1.07 & & 1.71 & \\
\hline FeOT & 4.60 & 4.69 & 4.32 & 4.49 & 6.12 & 6.57 & 6.70 \\
\hline \multicolumn{8}{|l|}{ Trace elements (ppm) } \\
\hline V & 44 & 59 & 94 & 83 & 140 & 199 & 129 \\
\hline $\mathrm{Ti}$ & 1367 & 1613 & 1793 & 1619 & 2794 & 3539 & 2098 \\
\hline $\mathrm{Cr}$ & 648 & 320 & 1100 & 553 & 169 & 85 & 332 \\
\hline $\mathrm{Ni}$ & 251 & 233 & 271 & 216 & 94 & 159 & 153 \\
\hline $\mathrm{Sr}$ & 103 & 136 & 126 & 133 & 94 & 149 & 110 \\
\hline $\mathrm{Zr}$ & 14 & 23 & 27 & 16 & 16 & 26 & 18 \\
\hline $\mathrm{Ba}$ & 1 & 13 & 13 & 8 & 3 & 14 & 9 \\
\hline Co & & & & 34 & & & 42 \\
\hline $\mathrm{Cu}$ & & & & 85 & & & 116 \\
\hline $\mathrm{Zn}$ & & & & 23 & & & 39 \\
\hline Sc & 17.0 & 18.1 & 24.3 & 19.8 & 43.2 & 37.4 & 34.9 \\
\hline $\mathrm{Y}$ & 5.5 & 7.2 & 7.5 & 6.2 & 12.6 & 14.9 & 9.9 \\
\hline \multicolumn{8}{|l|}{ Rare earths (ppm) } \\
\hline La & 0.79 & 0.79 & 0.87 & 0.65 & 0.82 & 0.31 & 0.59 \\
\hline $\mathrm{Ce}$ & 3.08 & 3.30 & 3.28 & 2.56 & 3.67 & 2.49 & 2.69 \\
\hline Nd & 1.53 & 1.82 & 2.04 & 1.22 & 2.58 & 2.23 & 1.65 \\
\hline $\mathrm{Sm}$ & 0.54 & 0.64 & 0.73 & 0.47 & 0.99 & 0.93 & 0.67 \\
\hline $\mathrm{Eu}$ & 0.41 & 0.45 & 0.44 & 0.36 & 0.53 & 0.65 & 0.47 \\
\hline Gd & 0.77 & 0.91 & 1.08 & 0.74 & 1.56 & 1.60 & 1.13 \\
\hline Dy & 0.85 & 1.12 & 1.21 & 0.97 & 2.01 & 2.32 & 1.53 \\
\hline $\mathrm{Er}$ & 0.56 & 0.74 & 0.75 & 0.64 & 1.25 & 1.44 & 0.97 \\
\hline $\mathrm{Yb}$ & 0.55 & 0.69 & 0.72 & 0.58 & 1.17 & 1.33 & 0.92 \\
\hline $\mathrm{Lu}$ & 0.07 & 0.08 & 0.09 & 0.09 & 0.16 & & 0.13 \\
\hline \multicolumn{8}{|l|}{$\begin{array}{l}\text { Isotopes } \\
{ }^{87} \mathrm{Sr} /{ }^{86} \mathrm{Sr}\end{array}$} \\
\hline
\end{tabular}

served in each hole, some of which are composite and zoned inward from dunite to wehrlite to clinopyroxenite and others from dunite to wehrlite to clinopyroxenite to gabbro as traverses are made from the margin to the center of the vein (Fig. 9). They may show strong compositional and mineral chemistry zoning to more fractionated compositions as the interior of the vein is approached. The dunitic zones generally form sheaths or halos around the pyroxenite and gabbroic veins and often contain magmatic clinopyroxene and orthopyroxene. In other cases, the zones affected by clinopyroxene addition appear to be broader (e.g., the zone of $\mathrm{CaO}$ enrichment within residual harzburgites between 100 and 120 mbsf in both holes) and would tend to indicate a zone of concentrated porous flow.

The veins within the mantle sections sampled during Leg 153 appear to reflect a crystallization order that is not expected for low-pressure MORB crystallization. The low-pressure crystallization order for MORB involves a sequence of olivine \pm spinel, olivine + plagioclase, and olivine + plagioclase + clinopyroxene. Based on the physical evidence of composite vein zonation (Fig. 9) and the mineral chemistry variations across the veins within Holes 920B and 920D, clear evidence of a crystallization orders of olivine, then olivine + clinopyroxene ( \pm orthopyroxene) or alternatively of olivine, then olivine + clinopyroxene $( \pm$ orthopyroxene $)$, then olivine + clinopyroxene + plagioclase $\pm \mathrm{Fe}-\mathrm{Ti}$ oxides \pm sulfides exist (also see plates for Holes 920B and 920D; Shipboard Scientific Party, 1995). Olivine + cli- nopyroxene or clinopyroxene + orthopyroxene coprecipitation in the absence of plagioclase is not predicted when MORB melts crystallize at pressures less than $\sim 10 \mathrm{kbar}$ (e.g., Elthon et al., 1982; Grove et al., 1992). Composite veins that show plagioclase at the center probably crystallized at lower pressures than $10 \mathrm{kbar}$, but the fact that clinopyroxene precedes plagioclase in the crystallization sequence documented would not be typical of MORB crystallization. Certainly, the order is more typical of higher pressure crystallization of MORB melts. The fact that many of the veins appear undeformed, however, would not support crystallization at these significant and high pressures, unless the base of the mechanical lithosphere is $30 \mathrm{~km}$ deep. This is considered unlikely in the MARK region based on seismic results of Toomey et al. (1985). The order of crystallization may, however, be explained in a number of ways other than very high-pressure crystallization, if the melts that crystallize involve near-fractional melts. One explanation is that the wehrlites and pyroxenites solidify at lower pressures (3-6 kbar) from melts with high $\mathrm{CaO} / \mathrm{Al}_{2} \mathrm{O}_{3}$ ratios that are unlike pooled MORB melts. They would represent near-fractional or poorly mixed melts that solidify at lower pressures. Another explanation may be that they are the products of previously fractionated mantle-derived melts that have reacted with the mantle to form wehrlite and pyroxenite.

As previously noted, most residual harzburgites show excess depletion in $\mathrm{CaO}$ based on the extent of melting predicted by $\mathrm{MgO}$ and 


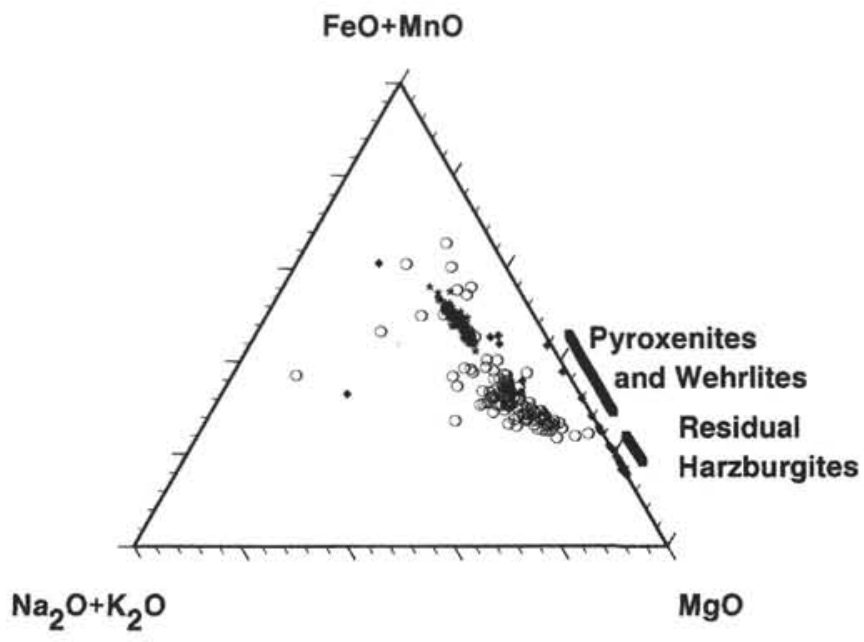

Figure 6. AFM triangular plot for ultramafic and plutonic rocks recovered from the MARK region during Leg 153. Also shown are the compositions of MARK glass (sources in referred to in Fig. 3). Samples from Sites 921, 922 , and 923 are indicated by open circles, Site 920 samples are indicated by solid diamonds, and basaltic glass samples are indicated by solid stars. The data span a wide range from both primitive to fractionated and show a strong iron enrichment trend. The compositional range is similar to that of most ophiolite complexes.

$\mathrm{Al}_{2} \mathrm{O}_{3} \cdot \mathrm{CaO} / \mathrm{FMM}$ vs. $\mathrm{Al}_{2} \mathrm{O}_{3} / \mathrm{FMM}$ plots show unusual behavior in that $\mathrm{CaO}$ abundances generally fall off the melting trend to significantly more depleted values than predicted by the melting model. This was also noted based on the shipboard studies (Shipboard Scientific Party, 1995). Several workers have noted this $\mathrm{CaO}$ depletion in abyssal peridotites (e.g., Falloon and Green, 1987; Hess, 1994) and have attributed it to mobilization during serpentinization. Shipboard studies show, however, that clinopyroxene, the main $\mathrm{CaO}$-bearing phase, is the most resistant to alteration and thus extensive mobilization during serpentinization may not completely explain this depletion trend. The lack of extensive mobilization of $\mathrm{CaO}$ is also consistent with the experimental data of Janecky and Seyfried (1986), which showed that the reaction rates of olivine:orthopyroxene:clinopyroxene during $300^{\circ}$ lherzolite-seawater experiments were approximately $1.0: 1 \cdot 0: 0.1$ and that very little diopside actually dissolved during the experiments. Although serpentinization can be a causative factor of $\mathrm{CaO}$ mobilization in the residuum, lherzolite-seawater experimental data reported by Janecky and Seyfried (1986) indicate that $\mathrm{Ca}$ concentrations in the reacted solutions progressively decrease, rather than increase. This indicates that there should be a net addition of $\mathrm{Ca}$ during serpentinization, rather than the depletion observed in most samples. Shipboard petrographic data generally confirm that there is a correlation between $\mathrm{CaO}$ content and the modal clinopyroxene in samples, but that the majority of MARK samples are very depleted in clinopyroxene (Shipboard Scientific Party, 1995).

The average modal content of clinopyroxene in residual peridotites is $2.5 \%$. Many harzburgites from Holes $920 \mathrm{~B}$ and $920 \mathrm{D}$ contain little or no clinopyroxene. Part (perhaps up to $1 \%-2 \%$ ) of the clinopyroxene of the rock mode observed is also likely to have exsolved from orthopyroxene during ascent and cooling (Lindsley and Anderson, 1983). The model of Pearce and Parkinson (1993) would predict significant remaining clinopyroxene $(3 \%-5 \%)$ in the MARK samples based on $\mathrm{Al}_{2} \mathrm{O}_{3} / \mathrm{FMM}$ and $\mathrm{MgO} / \mathrm{FMM}$ abundances and experimental data. This is because, based on the wt $\% \mathrm{MgO}$ and $\mathrm{Al}_{2} \mathrm{O}_{3}$, the residual mantle should have never approached temperatures high enough to reach the diopside-out curve by simple melting. This is consistent with the high $\mathrm{Na}_{2} \mathrm{O}$ abundances in basalts and our trace-element modeling (see below) that indicate Fmax of $12 \%-15 \%$ for ba-
A

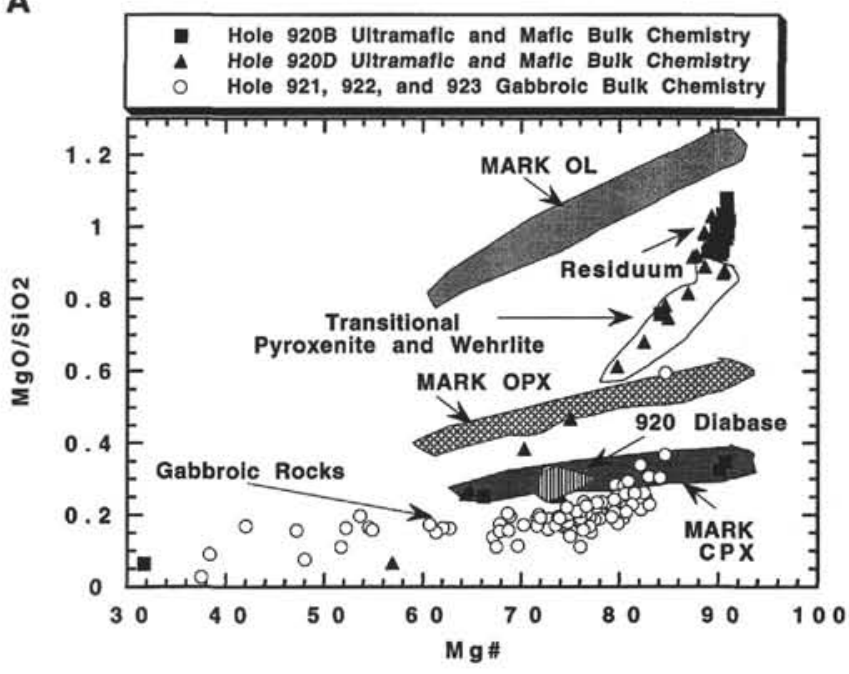

B

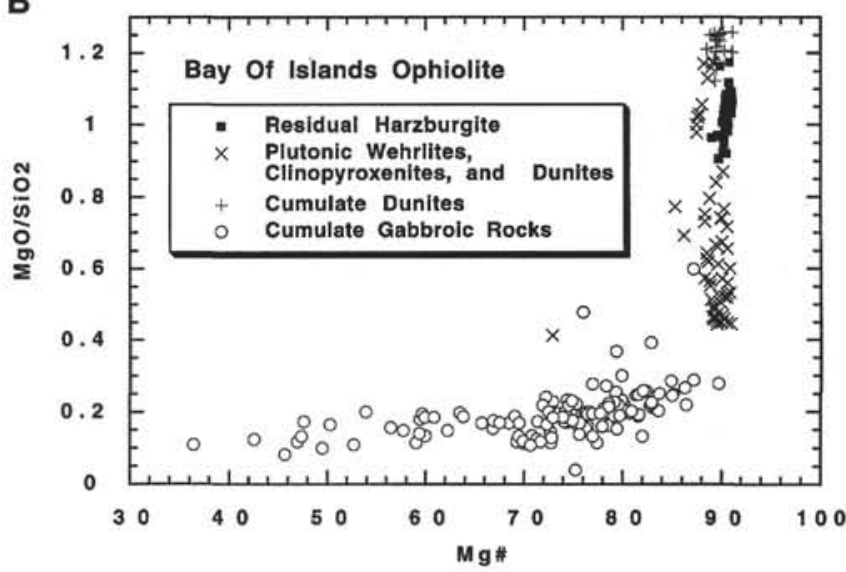

Figure 7. A. Bulk-rock $\mathrm{MgO} / \mathrm{SiO}_{2}$ vs. $\mathrm{Mg} \#$ for both peridotites and gabbroic rocks from Holes 920B and 920D (solid symbols), and gabbroic rocks from Holes 921 to 923 (open symbols). The range of compositions of mineral phases (olivine, clinopyroxene, orthopyroxene) documented for the core in the MARK area is also shown. Site 920 diabase compositions are also plotted for reference. $\mathrm{B}$. $\mathrm{MgO} / \mathrm{SiO}_{2}$ vs. $\mathrm{Mg} \#$ for cumulative gabbros, cumulate dunites, wehrlites, clinopyroxenites, and residual harzburgites for the Bay of Islands Ophiolite Complex (Casey et al., 1981). There is a strong similarity between the compositional range of samples in both suites. Ultramafic and mafic transition cumulates in the MARK core appear to be on average more strongly fractionated and show a trend from residual harzburgite compositions toward low-Mg\# oxide gabbro samples. (Data sources include Elthon et al., 1984; Komor et al., 1985a, 1987; J.F. Casey, unpubl. data).

salts and Fmax of $15 \%-20 \%$ for residual peridotites at the top of the melting column, which are significantly below the $25 \%$ considered necessary for the diopside out point.

An alternative explanation to serpentinization for the observed $\mathrm{CaO}$ depletion could involve melt-wallrock reactions during channelized flow or melt-matrix reactions during porous flow of melts that lead to early dissolution of clinopyroxene from the residuum, thus depleting the residuum in $\mathrm{CaO}$. During near-fractional melting, the melting column is an open system to melts that are generated deeper in the melting column. These melts are likely to be different in composition (more enriched in incompatible elements) than melts generated in the shallow mantle. Although these melts may be in equilibrium with clinopyroxene deeper in the column at their source, they are unlikely to be equilibrium with clinopyroxene as they move to shallower levels. This is because the olivine-phase volume expands with 
decreasing pressure, at the expense of the phase volumes of clinopyroxene and orthopyroxene (e.g., Bender et al., 1978). The positions of the olivine-orthopyroxene-clinopyroxene or olivine-plagioclaseclinopyroxene cotectics shift away from olivine with decreasing pressure (Bender et al., 1978). A melt saturated in clinopyroxene at its source will become undersaturated with respect to clinopyroxene as it passes through a previously depleted mantle lherzolite.

As melts generated deep in the melting column ascend and travel through the shallow melting column, they may react with the mantle by dissolving clinopyroxene and producing olivine + orthopyroxene to create harzburgite (e.g., Kelemen et al., 1992). They are also likely to maintain $\mathrm{Fe} / \mathrm{Mg}$ exchange equilibrium with olivine, because diffusion rates of $\mathrm{Fe}$ and $\mathrm{Mg}$ in olivine are high (Hess, 1992). This would lead to early depletion of the mantle in $\mathrm{CaO}$, but not necessarily in $\mathrm{Al}_{2} \mathrm{O}_{3}$, if olivine and orthopyroxene crystallize simultaneously along

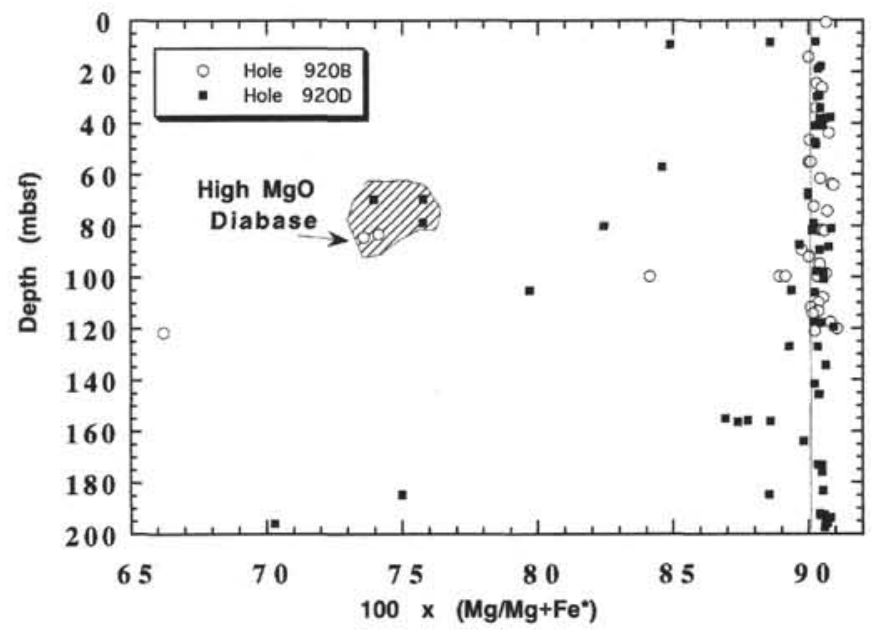

Figure 8. Downhole variations in bulk-rock Mg\#s: Hole 920B (open circles), and Hole 920D (solid squares). Note the abundances of samples with low $\mathrm{Mg} \#$ s below 90 that are atypical of residual mantle. These samples include pyroxenites, wehrlites, dunites, gabbroic, and metagabbroic rocks with intrusive contacts with the adjacent residual harzburgite. Also shown are 5 diabasic samples from each hole, indicated by enclosure within the striped field. a zone of channelized flow. Preferential dissolution of clinopyroxene eventually will produce a clinopyroxene-saturated melt. Melts that are always near saturation in clinopyroxene may crystallize clinopyroxene during their ascent initially in the absence of plagioclase, and possibly aided by moderate pressure cooling and fractionation (e.g., Elthon et al., 1982, 1984a; Casey et al., 1983; Tormey et al., 1987; Elthon, 1987). This may explain the late wehrlite and clinopyroxenite veins that intrude the residual mantle as it cools and becomes part of the mechanical lithosphere.

Higher pressure ( $>10 \mathrm{kbar}$ ) fractionation of these melts could lead to the early crystallization of olivine, olivine + clinopyroxene, olivine + clinopyroxene + orthopyroxene, and localized refertilization at deeper levels. The clinopyroxenes in these veins often have high $\mathrm{Mg \# s,} \mathrm{outside} \mathrm{the} \mathrm{range} \mathrm{predicted} \mathrm{at} \mathrm{lower} \mathrm{pressures} \mathrm{(Elthon} \mathrm{et} \mathrm{al.,}$ 1982; Grove et al., 1992). The significance of high Mg\# (92-82) clinopyroxene in these pyroxenite veins may indeed be that they indicate moderately high pressures of crystallization (Elthon et al., 1982). However, a perplexing feature of these veins is the lack of significant ductile deformation of the pyroxenites, if one is to accept the highpressure origin. Also, the mechanical lithosphere would have to be approximately $30 \mathrm{~km}$ thick. Some of the dikes also include plagioclase and oxides as fractionating phases, and they would necessarily form at lower pressures. Plagioclase does not crystallize until after olivine and clinopyroxene based on lithological zoning, and likewise most of these dikes have not undergone significant high-temperature ductile deformation. This would tend to indicate that significantly lower pressures of origin than $10 \mathrm{kbar}$ should be considered, at least for some and possibly all veins.

In localized zones in Holes 920B and 920D, the earliest veins appear to have been saturated in clinopyroxene at moderate to low pressures, and have locally refertilized the mantle with wehrlite, websterite, and clinopyroxenite veins. More diffuse zones of harzburgite contain magmatically twinned clinopyroxene and are enriched in $\mathrm{CaO}$ (e.g, the zone between 100 and 120 mbsf as previously discussed). Presumably these are the result of melts that impregnate sometime after the main phase of melting, mantle depletion, and deformation as the mantle makes the transition from asthenosphere to lithosphere and melts become more strongly channelized. The overall heterogeneity in the composition of the residual harzburgites may, in part, be the result of the combined processes of melting, early localized dissolution of pyroxene and olivine precipitation, and later en-
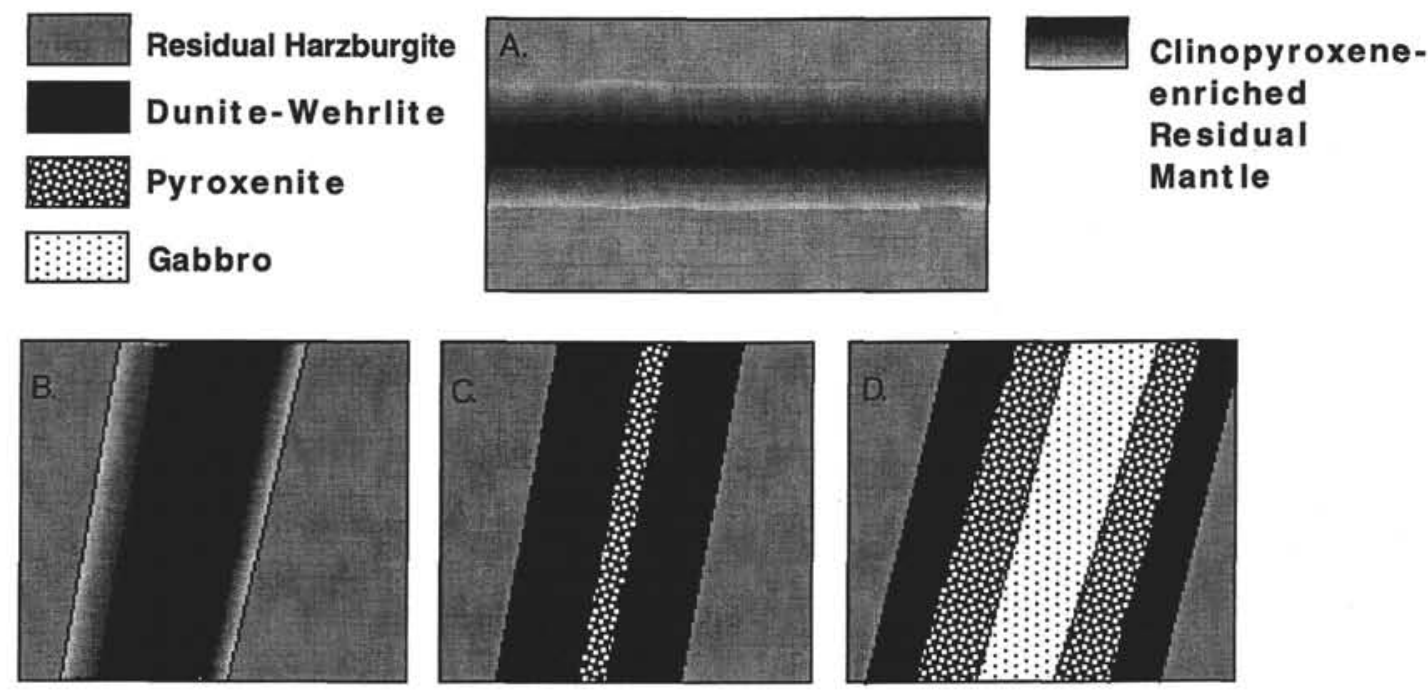

Figure 9. Types of melt channels documented within the residual harzburgites. A. Diffuse zone of harzburgite enriched in clinopyroxene (lherzolites) characterized by clinopyroxene with magmatic twins. B. Dunite-wehrlite veins with diffuse contacts with the adjacent residual harzburgite. C. Composite dunite-wehrlite-pyroxenite veins. D. Composite dunite-wehrlite-pyroxenite-gabbro vein. Note that the crystallization order is typically olivine, olivine + clinopyroxene, or olivine, olivine + clinopyroxene, olivine + clinopyroxene + plagioclase from the margin to the interior of the vein. 

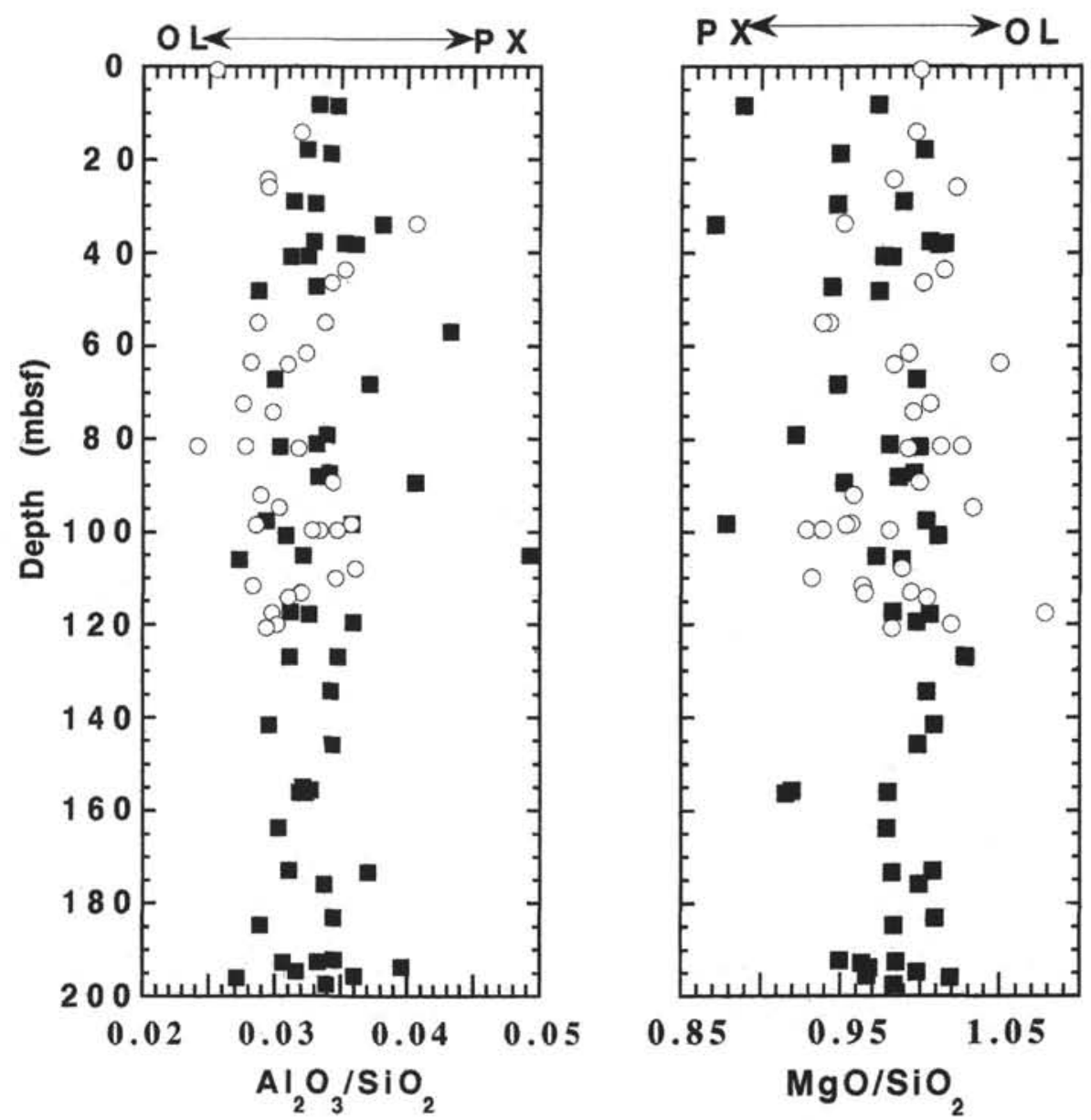

Increasing Modal CPX

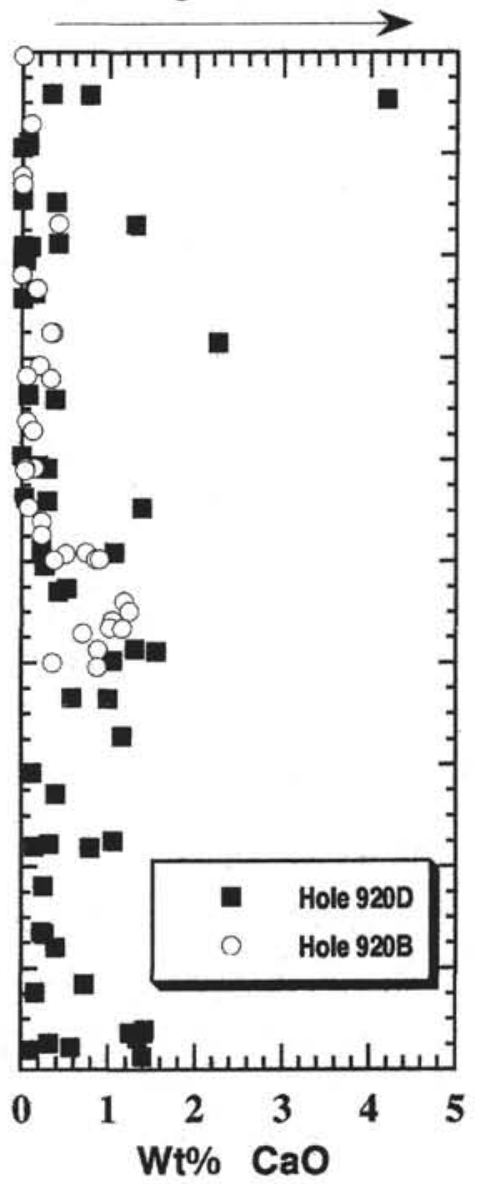

Figure 10. Downhole variations in bulk-rock $\mathrm{Al}_{2} \mathrm{O}_{3} / \mathrm{SiO}_{2}, \mathrm{MgO} / \mathrm{SiO}_{2}$, and $\mathrm{CaO}$ for Holes $920 \mathrm{~B}$ and $920 \mathrm{D}$. Both $\mathrm{MgO} / \mathrm{SiO}_{2}$ and $\mathrm{Al}_{2} \mathrm{O}_{3} / \mathrm{SiO}_{2}$ will vary as indicated with modal the modal olivine $(\mathrm{OL})$ to pyroxene $(\mathrm{PX})$ ratio and $\mathrm{CaO}$ will tend to be enriched in clinopyroxene-rich harzburgites and wehrlites. Note zones of pyroxene enrichment and clinopyroxene (CPX) enrichments within both cores. $\mathrm{CaO}$ enrichment between 100 and 130 mbsf correlates within both holes. The sample set would appear to indicate significant modal variations in ultramafic rocks within the holes.

richment during clinopyroxene crystallization. Channelization of melts would allow the heterogeneity to be maintained and preserved.

To illustrate the enriched to depleted trace-element signatures of some of the pyroxenites, extended trace-element plots are shown in Figure 15. These plots show that some of the pyroxenite veins are significantly enriched in REEs and other trace elements, with abundances either close to or higher than that of MARK basalts. The most enriched patterns are similar to those of some oxide gabbros and silicic rocks found with the gabbroic sections drilled during Leg 153 (see below). Assuming the veins are cumulate in nature, melts in equilibrium with these veins calculated based on the modal abundances and bulk mineral-melt distribution coefficient $(\mathrm{Kd})$ show that melts would have ranged from depleted to ultra-enriched in LREEs with high $\mathrm{La} / \mathrm{Yb}$ ratios relative to MARK basalts. Frey (1989) showed that pyroxenites and wehrlites from continental xenoliths show an equally broad range in variability. Another distinctive feature of these samples is that they show marked negative $\mathrm{Sr}$ and $\mathrm{Ti}$ anomalies, indicating that the melts that produced them have likely significantly fractionated through crystal separation or melt wall-wallrock fractionation processes during their ascent towards the surface. Meltwallrock reaction or extensive fractionation could lead to these depletions. Negative $\mathrm{Sr}$ anomalies in melts are typically the result of fractionation or reaction with plagioclase, and negative $\mathrm{Ti}$ anomalies are typically the result from Fe-Ti oxide precipitation. Extensive melt reaction with olivine and orthopyroxene during porous flow through the mantle could also aid in the formation of negative anomalies. In particular, olivine can cause fractionation of $\mathrm{Sr}$ and $\mathrm{Ti}$ with respect to the REE. It is also noteworthy that the most enriched samples have positive $\mathrm{Zr}$ anomalies typical of zircon accumulation or mantle melts that form as the result of very low extents of mantle melting. The most enriched veins are thus interpreted to possibly represent the products of low-percentage mantle melts that have either undergone significant crystal-liquid fractionation, reacted with olivine and orthopyroxene, or result from the reaction of evolved melts with the depleted residual mantle. Very similar veins have been reported from the $15^{\circ} 20^{\prime} \mathrm{N}$ region by Cannat and Casey (1995).

The enriched and fractionated nature of melts from which these veins are derived is also reflected in the mineral chemistries of clinopyroxene and olivine. For example, wehrlitic veins such as those in Sample 920D-18-3, 93-98 cm (Fig. 16), have olivine compositions that typically range from $\mathrm{Fo}_{91}$ near the contact with harzburgite to $\mathrm{Fo}_{83}$ towards the center of the vein. Likewise, the $\mathrm{Mg} \# \mathrm{~s}$ of clinopyroxene in wehrlite and pyroxenite from the same vein typically span a range from 91 to 84 . Samples 920D-7R-1, 76-83 cm, and 920D$10 \mathrm{R}-2,48-55 \mathrm{~cm}$, show a similar range in composition from $\mathrm{Mg} \# \mathrm{~s}$ of 91 to 84 . This is also reflected in the bulk-rock Mg\# (e.g., Table 2). Melts calculated to have been in equilibrium with clinopyroxene in these veins have $\mathrm{Na}_{2} \mathrm{O}$ abundances that range from those that are ultradepleted with respect to MARK glasses to those that are more enriched than MARK glasses (Fig. 17). These melts could not have 


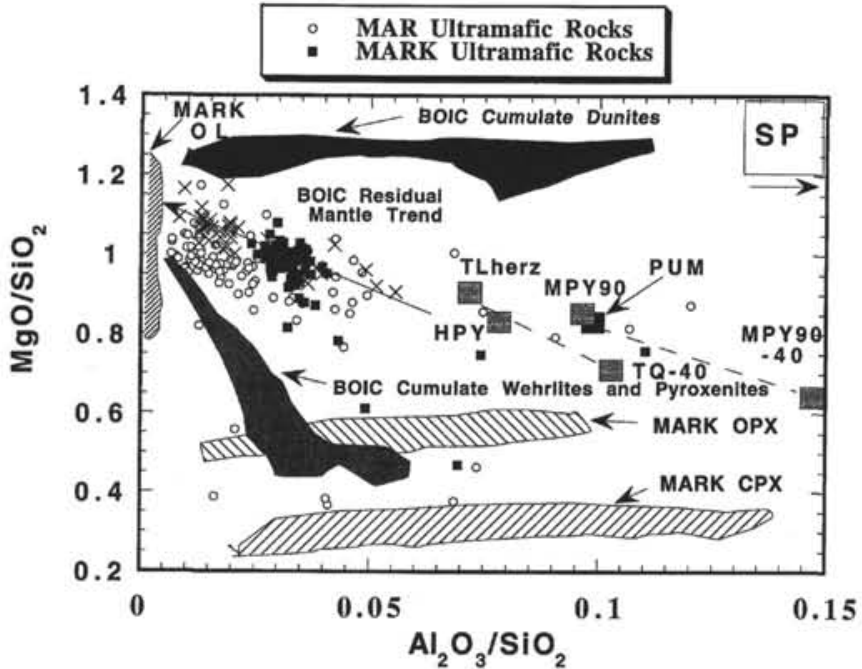

Figure 11. Comparisons of bulk-rock $\mathrm{MgO} / \mathrm{SiO}_{2}$ vs. $\mathrm{Al}_{2} \mathrm{O}_{3} / \mathrm{SiO}_{2}$ for Holes $920 \mathrm{~B}$ and 920D residual and cumulate peridotites with all Mid-Atlantic Ridge (MAR) residual and cumulate peridotites, BOIC residual harzburgites and lherzolites (crosses), and proposed compositions of MORB fertile mantle. Also shown are various estimates of MORB mantle (Falloon and Green, 1987), including Tinaquillo Lherzolite (TLHERZ), MORB Pyrolite (MPY90), Hawaiian Pyrolite (HPY), and Primitive Upper Mantle (PUM). Also shown are their adjusted compositions used in sandwich melting experiments for TLHERZ and MPY-90 (i.e., TQ-40 and MPY-90-40). The ranges of compositions of olivine, clinopyroxene, and orthopyroxene documented for all MARK peridotites and gabbroic rocks are also shown. Peridotite pyroxenes are enriched in $\mathrm{Al}_{2} \mathrm{O}_{3} / \mathrm{SiO}_{2}$ ratios, whereas gabbroic pyroxenes are commonly more depleted. The residual mantle trend for the BOIC (J.F. Casey, unpubl. data) is thought to be near primary. Note that most abyssal peridotites collected from the seafloor weathering zone have lower $\mathrm{MgO}$ / $\mathrm{SiO}_{2}$ ratios than MARK residual peridotites and BOIC residual peridotites. This is because most peridotites have undergone $\mathrm{MgO}$ extraction because of seafloor weathering processes (Snow and Dick, 1995). The MARK harzburgites lie along the BOIC residual mantle trend, which is similar to the trend defined by continental xenoliths (Jagoutz et al., 1979). Pyroxenites, lherzolites, wehrlites, and gabbroic rocks from Holes 920B and 920D have significantly lower $\mathrm{MgO} / \mathrm{SiO}_{2}$ than the bulk of the MARK residual peridotites. Also shown for comparison is the field of BOIC bulk-rock compositions for cumulate dunite, wehrlites, and pyroxenites (Komor et al., 1985a; J.F. Casey, unpubl. data).

been parental to MARK glasses, but could represent the melt-wallrock reaction products of highly fractionated melts or the solidification of a range of near-fractional or poorly mixed melts that have later pooled and hybridized to form parents similar to MARK primitive glasses. High-Mg\# pyroxenes could result from early crystallization of clinopyroxene from near-fractional melts or reaction of the melt with and buffering by the ultramafic wallrock.

\section{Discussion}

Holes 920B and 920D from the ultramafic massif on the western rift valley wall in the MARK region are dominantly residual harzburgites depleted by partial melting, the extent of which appears to range from $15 \%$ to $20 \%$ based on modeling of a source composition similar to MORB pyrolite (MPY-90) or FMM, or $10 \%-15 \%$, if a more depleted source such as Tinaquillo Lherzolite is chosen. The mantle section is far from a homogeneously depleted residuum. The residuum has generally been depleted in $\mathrm{CaO}$ more than predicted by a simple melting relation, perhaps by the dissolution of clinopyroxene as melts generated deeper in the melting column migrate through a residuum that resides higher in the melting column. Mantle deple- tion during the main phase of melting was apparently followed by impregnation of various depleted to more enriched melts derived from deeper in the mantle column. Many of these melts appear to have had elevated highly incompatible element abundances and lead to localized enrichments in the residuum. Late fractionated melts saturated in olivine (dunite-rich zones) or olivine and clinopyroxene (dunitewehrlite-pyroxenite composite veins) appear to occupy channelized zones formed in the ascending mantle after it was incorporated in the mechanical lithosphere. Many of these veins appear parallel-sided, undeformed, and have igneous textures, as do some clinopyroxene grains in the adjacent residual mantle where melt has apparently impregnated the mantle matrix and added cumulus minerals.

Because the veins have escaped the high-temperature pervasive ductile deformation that affect the bulk of the residual mantle section, they are interpreted to be late postkinematic intrusive rocks into a mantle that has previously made the transition from asthenosphere to lithosphere. These intrusions undergo moderate- to low-pressure fractionation within the mantle (3-5 kbar), and in the extreme case can form zoned dunite-wehrlite-pyroxenite-gabbro-oxide gabbro composite veins. The early crystallization of high-Mg\# clinopyroxene before plagioclase is not expected from MORB crystallized at low to moderate pressures less than $10 \mathrm{kbar}$ (Elthon et al., 1982; Elthon, 1987; Grove et al., 1992) and remains enigmatic. The lack of extensive deformation does not argue for very high pressures of crystallization because, except for localized deformation, the mantle matrix and veins should have undergone deformation if it was transported such large distances to the surface or formed in the active melting column. The change from the usual order of crystallization (olivineplagioclase-clinopyroxene) to olivine-clinopyroxene-plagioclase can be achieved by different mechanisms at lower pressures (3-5 kbar). Although very high pressures of crystallization ( $>10$ kbar) could easily explain high-Mg\# clinopyroxenite and wehrlites that lack plagioclase, this is not considered likely because microearthquake studies in the MARK region would suggest that the base of the mechanical lithosphere resides at depths probably slightly in excess of $10 \mathrm{~km}$ (Toomey et al., 1988) and not the $30 \mathrm{~km}$ required for high-pressure crystallization ( $>10 \mathrm{kbar}$ ).

The existence of melts with very high $\mathrm{CaO} / \mathrm{Al}_{2} \mathrm{O}_{3}(\sim 0.9-1.1)$ could cause a reversal in the order of crystallization even at low pressures (i.e., 3-5 kbar), but these melts are unlike accumulated (or pooled) fractional melts (i.e., MORB), which tend to have lower $\mathrm{CaO} / \mathrm{Al}_{2} \mathrm{O}_{3}$. Such high-CaO/Al $\mathrm{O}_{3}$ melts within the mantle section could be explained by either extensive dissolution of clinopyroxene from the residual mantle during its passage or by a highly refractory near-fractional mantle melt formed in the last stages of melting at the top of the residual melting column. $\mathrm{CaO} / \mathrm{Al}_{2} \mathrm{O}_{3}$ will increase as a function of either increased mantle melting or preferential dissolution of clinopyroxene. The reversal of crystallization order of clinopyroxene and plagioclase can occur at pressures of as little as 3-5 kbar (i.e., if the $\mathrm{CaO} / \mathrm{Al}_{2} \mathrm{O}_{3}$ ratios of melts are very high). High- $\mathrm{Mg} \#$ clinopyroxenites and wehrlites from the Bay of Islands basal plutonic sections (Elthon and Casey, 1985) may be interpreted in a similar manner, as they appear to be derived from very ultradepleted, late-stage, near- fractional melts.

Alternatively, the veins may represent the crystallization and reaction products of highly fractionated melts solidified within magma chambers within the mantle. The melts and pyroxene compositions would be buffered by the mantle-wallrock composition via $\mathrm{Fe} / \mathrm{Mg}$ exchange, yet would be characterized by high concentrations of incompatible trace elements. This would also explain the common occurrence of zircon, apatite and Fe-Ti oxide crystallization within the core of some of these veins and possibly the negative $\mathrm{Sr}$ and $\mathrm{Ti}$ anomalies in trace-element patterns.

The veined mantle sections documented here may represent the transitional cumulate compositions typical of ophiolitic mantle-crust transition zones (e.g., Casey et al., 1981, 1983; Komor et al., 1985a; Nicolas, 1986) previously regarded as lacking within oceanic lithos- 

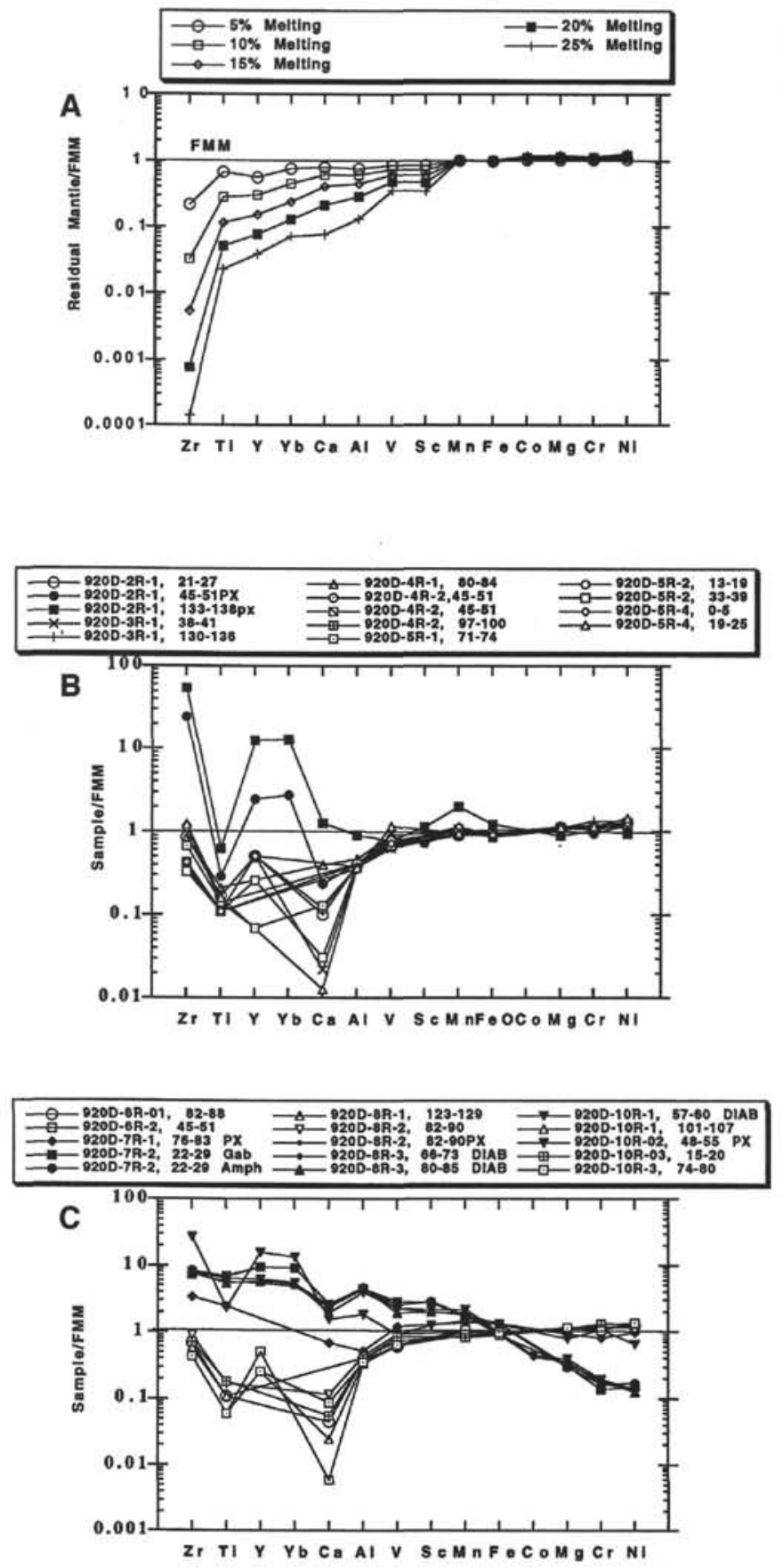
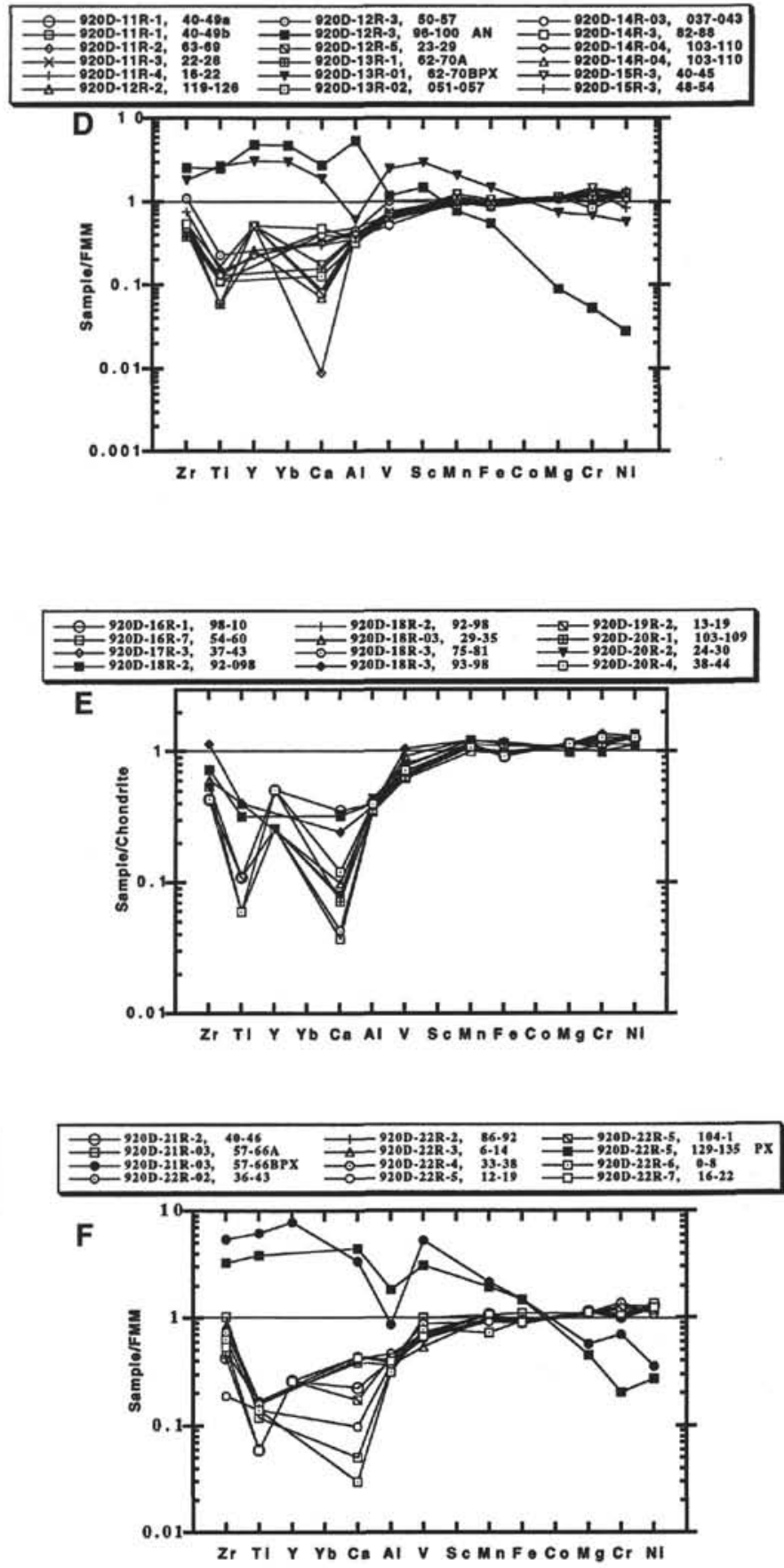

Figure 12. Spider diagrams showing elements listed by increasing compatibility (left to right) during mantle melting with samples normalized to a FMM source (Pearce and Parkinson, 1993). A. The composition of residual mantle (normalized to FFM) that is expected after 5\%, 10\%, 15\%, 20\%, and $25 \%$ melting of FMM. Note strong depletions of the most incompatible elements. B-F. Samples from Hole 920D show residual mantle compositions (sample numbers without labels = residual harzburgite), as well as cumulate pyroxenites (PX), gabbro (GAB), amphibolite (AMPH), and diabasic (DIAB) intrusions into the harzburgite. Note the apparent $\mathrm{U}$-shaped pattern for most harzburgite samples and the severe depletions of $\mathrm{CaO}$ in many of the samples. Also note the enriched character of the intrusive rocks with respect to the more incompatible elements.

pheric sections. These sections commonly include dunites, pyroxenites, and wehrlites containing high-Mg\# clinopyroxene (Elthon et al., 1982; Komor et al., 1985a). The volume of various veins in the mantle may be large, based our core observations (also see Shipboard Scientific Party, 1995; Cannat, Chatin, et al., this volume) and those from other ODP sites (Girardeau and Mercier, 1992), but they are generally disguised in the residual mantle matrix of oceanic samples. These transition-zone rocks may be more abundant near the center of second-order segment characterized by melt focusing when com- pared with major fracture zones (Dick, 1989) where melt withdrawal may predominate.

The refertilization of the mantle by veining and alteration of residual peridotites would appear to occur by the partial crystallization of melt fractions of various compositions as they travel through the mantle. The refertilization is envisaged to take place by the addition of cumulate products of these melts that transit toward the surface, rather than by entrapment, because many of the veins (e.g., pyroxenite and gabbroic veins) are not similar to melt compositions. Because 

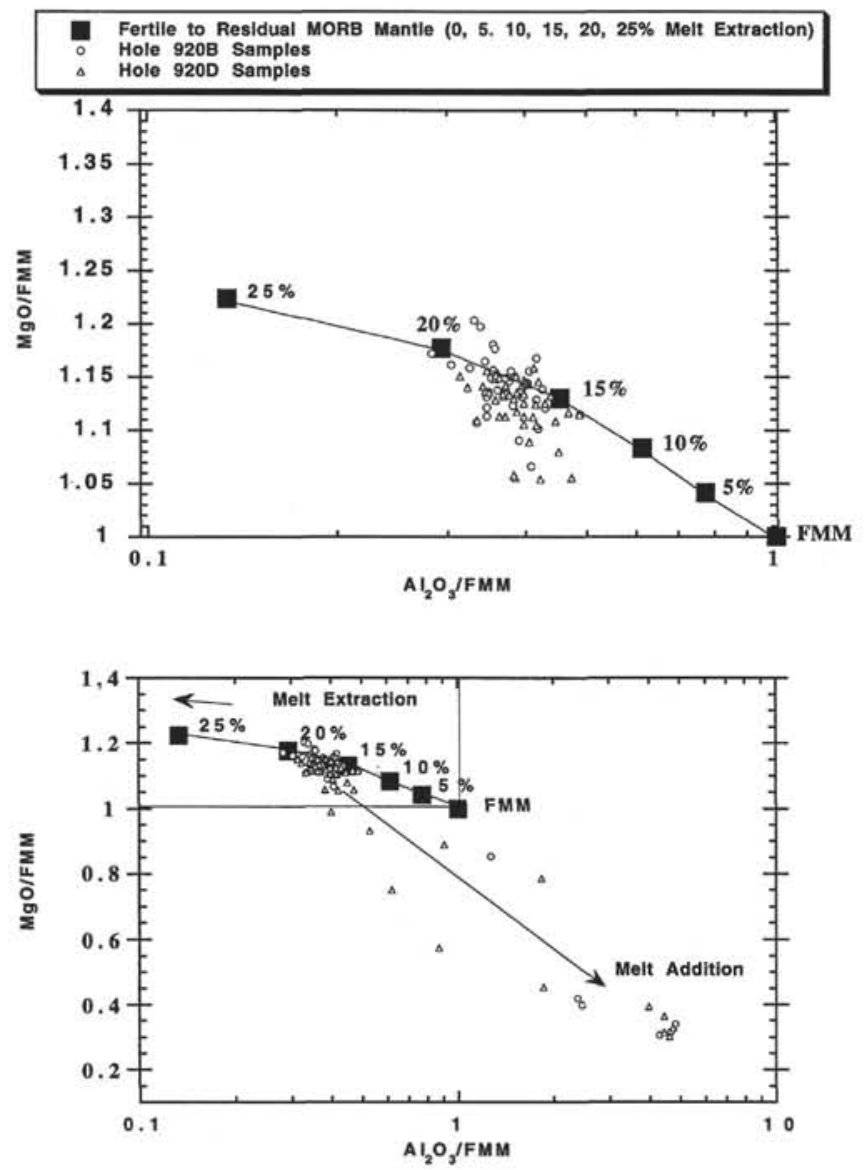

Figure 13. Bulk-rock melting trend as defined by Pearce and Parkinson (1993) for $\mathrm{MgO} / \mathrm{FMM}$ vs. $\mathrm{Al}_{2} \mathrm{O}_{3} / \mathrm{FMM}$ (upper diagram), showing the fertile to residual mantle melting trend after $0 \%-25 \%$ melt extraction at $5 \%$ intervals. Most of the residual mantle samples from Holes 920B and 920D plot between $15 \%$ and $20 \%$ of melting. $\mathrm{A}$ plot of $\mathrm{MgO} / \mathrm{FMM}$ vs. $\mathrm{Al}_{2} \mathrm{O}_{3} / \mathrm{FMM}$ (lower diagram) shows the same mantle melting trend, but includes a broader range of compositions. The intrusive ultramafic and gabbroic samples are plotted outside the mantle melting trend and are indicative of melt impregnation and accompanying cumulate formation within the residual mantle after melting.

the mantle at higher levels in the melting column is an open system to melts generated deeper, near-fractional melt compositions that produce these cumulate products may vary significantly from low-to high-fraction melt and from little to extensively fractionated. This differs significantly from other models of refertilization. Dick (1989) and Cordery and Phipps Morgan (1993) envisage refertilization to result from mixing MORB primary melt with residual harzburgite. The melt would become trapped and crystallize in situ within the residual mantle. Clinopyroxenite, wehrlitic, and gabbroic veins, and magmatic clinopyroxene within the residuum are unlikely to represent the composition of congealed trapped melts, because their bulk chemistry is unlike the composition of any melt. Significant refertilization as envisaged here appears more zonal and restricted to melt channels that produce clinopyroxene-enriched mantle and cumulate dunite-pyroxenite-gabbro veins. The production of cumulate clinopyroxene in the mantle has also been invoked by Elthon (1987) and Grove et al. (1992) based on compositional variations of basalts in the Cayman and MARK area, respectively, but the high Mg\#s of clinopyroxene (up to 93) and the lack of simultaneous crystallization of plagioclase is not predicted in these models. This crystallization was thought to occur in the mantle at pressures of 3-10 kbar, but it is suggested here that the crystallization of vein clinopyroxene in the MARK peridotite massif is likely to have occurred at lower pressures of 3-5 kbar in the mechanical lithosphere.

\section{BULK CHEMISTRY AND DOWNHOLE CRYPTIC GEOCHEMICAL VARIATIONS IN GABBROIC SECTIONS (SITES 921, 922, 923)}

The compositional variability of plutonic rocks, including gabbros, wehrlites, and pyroxenites recovered during Leg 153, can be observed in Figure 18, which shows a histogram of Mg\#s of all Leg 153 plutonic samples. Ultramafic intrusive rocks from Holes 920B and $920 \mathrm{D}$ are included in these plots, together with all gabbroic samples analyzed from Site 921 (Table 3), Site 922 (Table 4), and Site 923 (Table 5). The bulk of the samples lie in the $\mathrm{Mg} \#$ range from 70 to 85 , and the melts in equilibrium with these samples would tend to be similar to the main field of basalt glasses sampled from the MARK region ( $\mathrm{MgO} 6-8.5 \mathrm{wt} \%$ ). Mg\#s of the plutonic rocks, however, have a total range from $\sim 90$ to 30 and compositionally form as wide a spectrum as the combined samples analyzed from various ridge segments within the oceanic basins. Included in this plot are available analyses from the Southwest Indian Ridge and Site 735 (Meyer et al., 1989; Dick et al., 1991), the Mid-Atlantic Ridge (Tiezzi and Scott, 1980), and the Cayman Trough (Elthon, 1987) for comparison. Similarly, Figure 19 shows that the bulk compositions of the Leg 153 gabbros form a data set that covers spectrum of oceanic samples with a range of $\mathrm{CaO} /\left(\mathrm{CaO}+\mathrm{Na}_{2} \mathrm{O}\right)$ and $\mathrm{FeO}^{*}$ (total iron calculated as $\mathrm{FeO}$ ) content. In the following sections, the overall major- and trace-element characteristics of Site 921,922 , and 923 are briefly reviewed.

Trace elements reported range from those that are highly compatible to those that are highly incompatible during fractionation of a basaltic melt. Extended rare-earth spidergrams are presented for each sample analyzed in this study, with elements listed in order of presumed incompatibility during mantle melting (Sun and McDonough, 1989) and normalized to chondrite using the values of Anders and Grevesse (1989). Sample lithologies are listed with each analysis in Tables 3, 4, and 5 and are listed in the order of depth of recovery.

Trace-element abundance in gabbroic rocks are dependent on the composition of the parent melt from which the crystals solidified, the partitioning between melt and crystalline phases (Fig. 20), the modal mineralogy of the sample, and the percentage of melt that has been trapped within the crystal framework either as restite intercumulus magma or magma that has infiltrated through the cumulate network. Gabbroic rocks can potentially range from simple congealed melts, in which case they would have bulk compositions similar to melts, to adcumulates, in which case all melt has been excluded from the final solidification product and the bulk composition is the result of a mixture of fractionally crystallized phases (each having a homogeneous composition). Variable percentages of trapped melt will tend to shift compositions away from solid fractionally crystallized products towards melt compositions. Virtually all of the gabbroic samples analyzed are cumulates, as none of these compositions represent reasonable melt compositions, with the possible exception of the silicic endmembers. Highly incompatible element abundances are generally significantly more depleted in gabbroic samples than in equilibrium basaltic melts, demonstrating their cumulate nature. A simple approach in computing the percentage of trapped melt assumes a common parent and utilizes trends of basaltic liquids, bulk chemistry, mineral chemistry, and modal analyses coupled with the bulk partitioning behavior of incompatible trace elements (e.g., see Meyer et al., 1989), but this has not been attempted here because preliminary evidence shows some melts from which cumulates have crystallized may had significantly different parents from MARK basalts and each other. Quantitative estimates of retained melt will await additional detailed major- and trace-element phase-chemistry studies. Trace-element signatures, in most cases, do imply a certain percentage of trapped melt within these cumulates, and mineral zonation studies tend to confirm some melt porosity, intercumulus liquid fraction- 

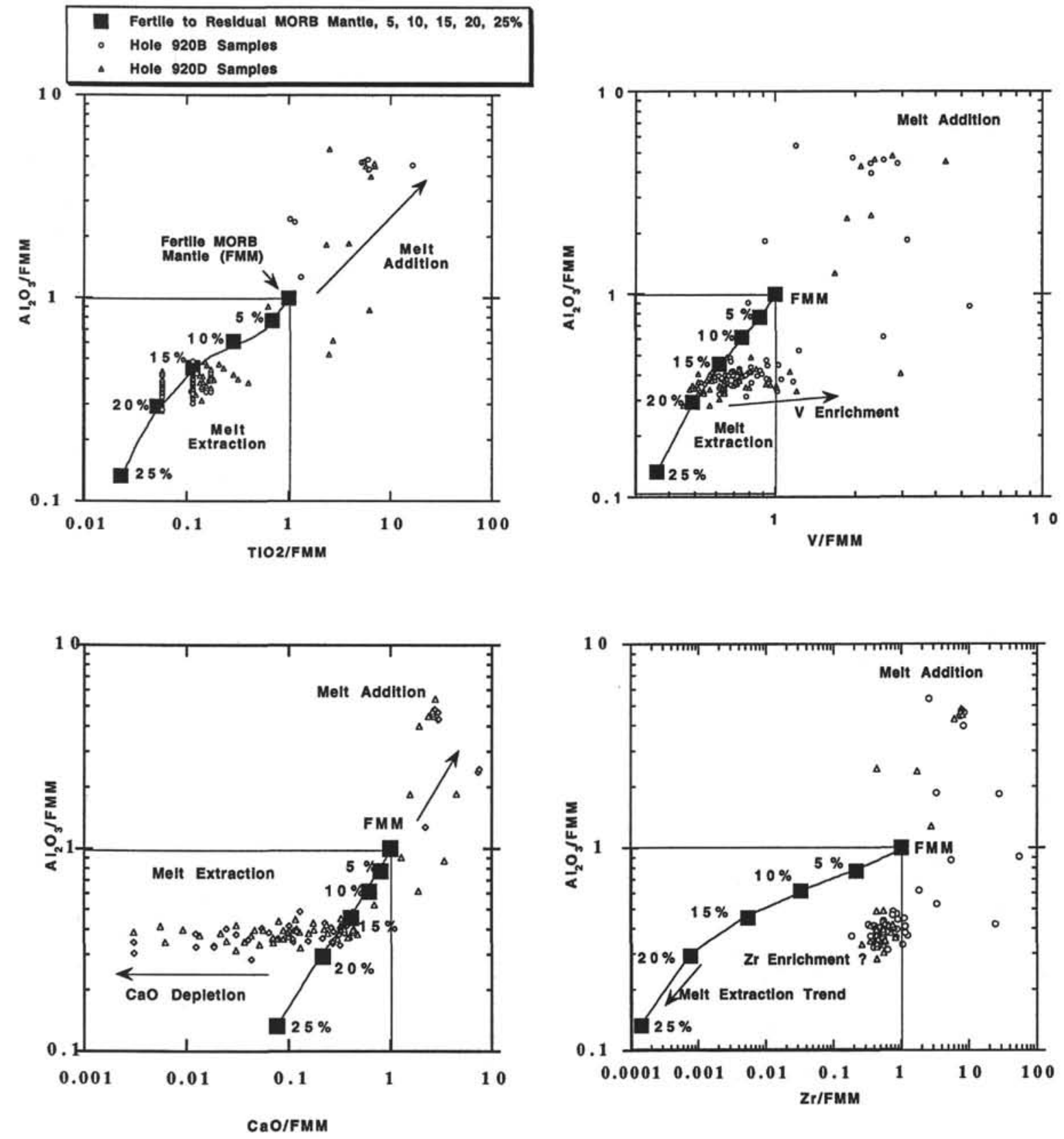

Figure 14. Bulk-rock melting trends as defined by Pearce and Parkinson (1993) for particular trace elements plotted vs. $\mathrm{Al}_{2} \mathrm{O}_{3} / \mathrm{FM}$. These show the fertile to residual mantle melting trend (solid squares) after $0 \%-25 \%$ melt extraction, shown at $5 \%$ intervals. $\mathrm{Al}_{2} \mathrm{O}_{3} / \mathrm{FMM}$ vs. $\mathrm{TiO}_{2} / \mathrm{FMM}, \mathrm{Al}_{2} \mathrm{O}_{3} / \mathrm{FMM}$ vs. V/FMM, $\mathrm{Al}_{2} \mathrm{O}_{3} / \mathrm{FMM}$ vs. $\mathrm{Zr} / \mathrm{FMM}$, and $\mathrm{Al}_{2} \mathrm{O}_{3}$ vs. $\mathrm{CaO} / \mathrm{FMM}$ plots are included. Incompatible elements tend to show enrichments relative to the concentrations predicted and may indicate enrichments caused by refertilization processes. The $\mathrm{CaO}$ plot shows the marked depletion of $\mathrm{CaO}$ from that expected during melting. The departure of $\mathrm{CaO}$ from the melting trend toward more depleted values may indicate preferential dissolution of clinopyroxene from mantle assemblage by throughgoing mantle melts. These melts are presumably derived from deeper in the subaxial melting column. The field of melt extraction and melt addition delineate rocks that form as the residue of melting and because of melt crystallization, respectively.

ation, and evolution. Based on the abundances of incompatible elements and mineral zoning in plagioclase, the majority of the samples studied thus far appear to have low trapped melt percentages (adcumulates), but orthocumulates to mesocumulates are present locally.

\section{Site 921}

Bulk-rock major-element, trace-element, and REE analyses are listed for 57 samples from Holes 921A, 921B, 921C, 921D, and $921 \mathrm{E}$. This gabbroic site shows the widest diversity of bulk composi- tions, with rock types ranging from troctolite, olivine gabbro, gabbronorite, oxide gabbro, to quartz diorite. Whole-rock $\mathrm{Mg \# s} \mathrm{range} \mathrm{from}$ 37 to 83 (Fig. 21), $\mathrm{SiO}_{2}$ from 45.37 to $61.23 \mathrm{wt} \%$, and $\mathrm{FeO} *$ from 3.2 to $13.7 \mathrm{wt} \%$. As previously noted, two diabases were analyzed from Site 921 (Fig. 22). Downhole plots of Mg\#s monitor quite accurately the Mg\#s of pyroxene and olivine within the gabbroic samples because modal variation in mafic phase proportions have little effect on

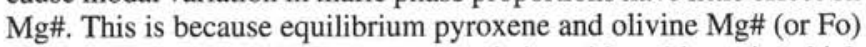
differ by less than $2 \%$. The exception is in oxide gabbros, in which case the lower $\mathrm{Mg} \#$ reflects, in part, the addition of iron oxides. In 


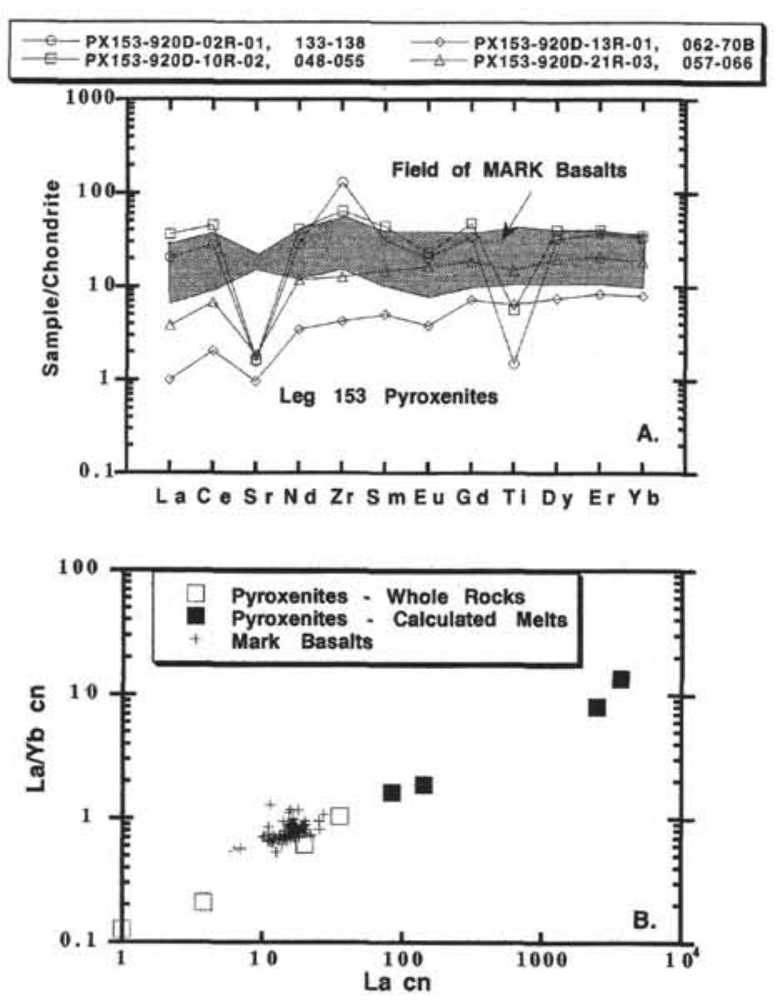

Figure 15. The upper diagram shows extended trace-element patterns for pyroxenites (websterites, clinopyroxenites) from Hole 920B and 920D. Also shown is the field for MARK basalts for comparison. Patterns show unusual enrichments in REE abundances and strong negative $\mathrm{Sr}$ and $\mathrm{Ti}$ anomalies and positive $\mathrm{Zr}$ anomalies in the most enriched melts. The lower diagram shows the whole-rock chondrite-normalized ( $\mathrm{cn}$ ) $\mathrm{La} / \mathrm{Yb}$ vs. chondrite-normalized $\mathrm{La}$ variations for the same samples and the calculated equilibrium melt composition based on modal abundances and $\mathrm{Kd}$ listed in Table 6, assuming, that is, that the pyroxenes are cumulate crystallization products of a melt. MARK basalt samples are also shown for comparison.

Figure 21, Mg\# is plotted vs. depth for all samples from Holes 921B, $921 \mathrm{C}, 921 \mathrm{D}$, and 921E. The figure shows that downhole variation is moderately well correlated from one hole to the next. The data show cryptic variation related to variations in magma compositions. Ca\# does not track magma evolution as well as $\mathrm{Mg} \#$ because it is affected not only by the anorthite (An) content of plagioclase, but by the modal abundance of clinopyroxene ( $20 \mathrm{wt} \% \mathrm{CaO})$. Understandably, the resolution of the Ca\# number in identifying magma cycles is significantly less.

There is a lack of intermediate compositions between evolved low- $\mathrm{Mg \# ,} \mathrm{SiO}_{2}$-rich samples and those with $\mathrm{Mg \# s}$ of $70-85$ that constitute the bulk of the samples. The quartz diorite and more felsic samples represent small veins and intrusions into the gabbro, as do certain oxide gabbros within each of the holes. We interpret the compositional gap to indicate that oxide gabbros and felsic veins may be the products of in situ intercumulus melt fractionation and eventual melt segregation to form small intrusive veins. The products of this extended fractionation are solidified as zoned rims on preexisting, higher temperature, cumulus crystals as the pore melt solidifies. The process is similar to orthocumulate addition to cumulus crystals as originally envisaged by Wager et al. (1960), and can lead to extensive fractionation to oxide gabbros and generation of felsic melts, such as quartz diorite. Zones in each hole characterized by oxide gabbros and felsic veins are usually also characterized by higher incompatible trace-element abundances, apparently indicating higher pore melt abundances during solidification (also see Agar et al., this volume). Mobilization of these late-stage melts is possibly synchronous with
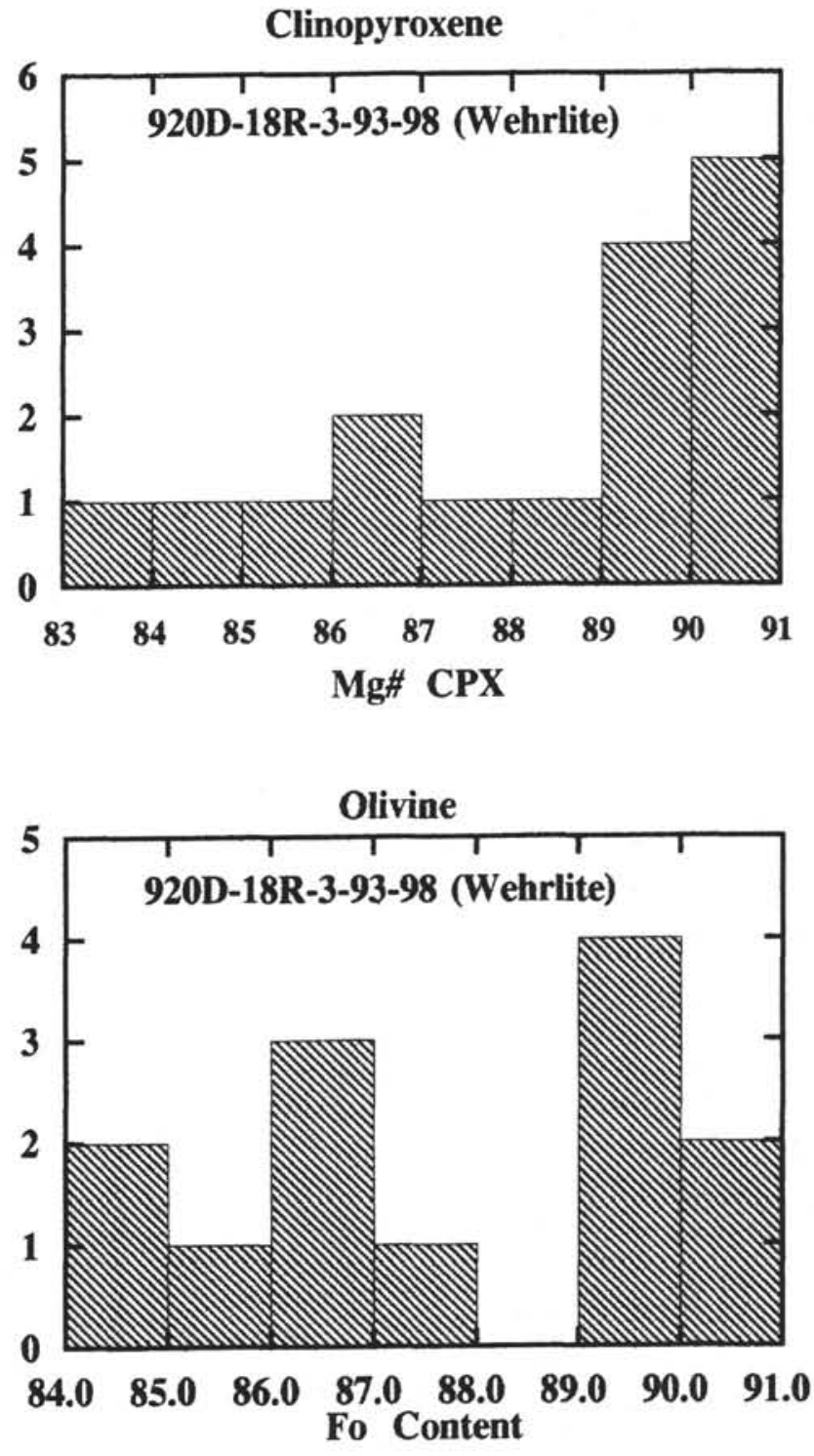

Figure 16. Histograms of the distribution of average $\mathrm{Mg} \#(100 \times \mathrm{Mg} /[\mathrm{Mg}+$ $\left.\mathrm{Fe}^{*}\right]$ ) composition for clinopyroxene (CPX) and Fo value for olivine within a single intrusive wehrlite (Sample 920D-18R-3, 93-98 cm), showing the wide variation in fractionation. The vertical axis represents the number of individual grains analyzed. Four analyses were averaged for each grain.

the development of shear zones within the crystal pile (Agar et al., this volume). Similar relations and processes have been proposed for oxide gabbros and felsic veins for the Bay of Islands Ophiolite gabbroic sections (Casey, 1980), for gabbroic sections recovered in Hole 735B in the Southwest Indian Ocean (Dick and Kelemen, 1991; Ozawa et al., 1991; Natlund et al., 1991), and the $15^{\circ} 20^{\prime} \mathrm{N}$ region of the Mid-Atlantic Ridge (Cannat and Casey, 1995).

Trace-element abundances (Figs. 22, 23) for Holes 921A, 921B, $921 \mathrm{C}, 921 \mathrm{D}$, and $921 \mathrm{E}$ generally vary with extent of fractionation, although there is wide scatter in their abundance at a given $\mathrm{Mg \#}$. The scatter in abundances tends to be widest at the more primitive end of the plutonic compositional spectrum. In part, these variations are attributed to modal variation, the percentage of trapped melt, and the existence of a range of melt compositions that vary between ultradepleted and highly enriched in incompatible elements with respect to MARK basalts. Several patterns emerge from spidergrams from gabbroic holes. In general, the REEs form smooth patterns with the exception of large positive Eu anomalies $(\mathrm{Eu} * / \mathrm{Eu}$ generally varies from 

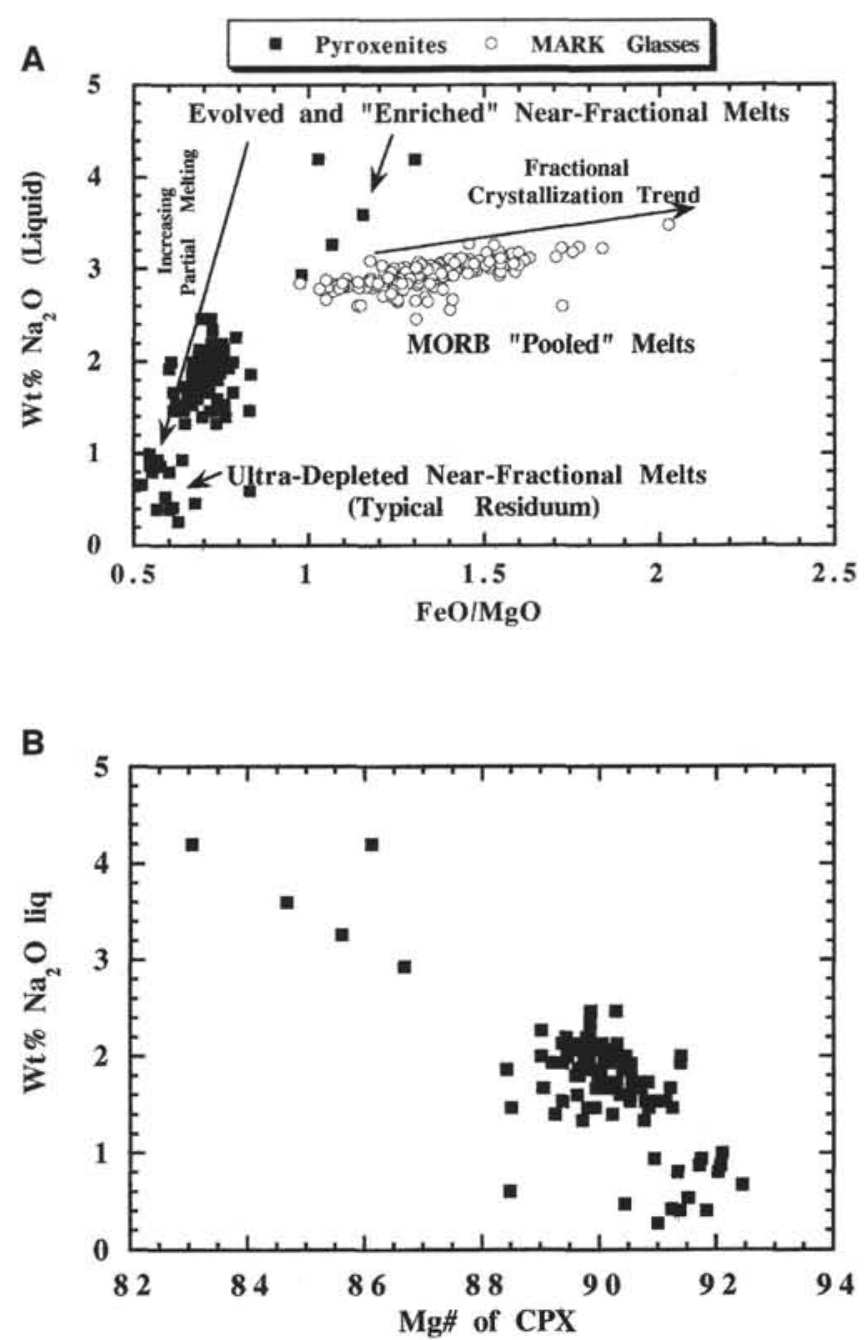

Figure 17. A. Plot of the $\mathrm{Na}_{2} \mathrm{O}$ and $\mathrm{FeO} / \mathrm{MgO}$ contents of melt that would be in equilibrium with clinopyroxene in several pyroxenites. Also shown are the array of MARK glass analyses (Bryan et al., 1994; Smithsonian Glass Catalog; and J.F. Casey, unpubl. data). Calculated melts range from ultradepleted to ultraenriched relative to MARK glasses. B. Plot of the Mg\# of clinopyroxene relative to the $\mathrm{Na}_{2} \mathrm{O}$ content of the melt in equilibrium with it. Note the very high $\mathrm{Mg \#}$ of clinopyroxenes. These are not expected to crystallize from MORB-like magmas at low pressures.

1 to 2.5), typical of plagioclase-bearing cumulates. Plagioclase-rich troctolites tend to exhibit LREE-enriched patterns, with $\mathrm{La} / \mathrm{Yb}$ ratios $>1$ and low overall abundances of heavy REE (HREE). Olivine gabbros tend to exhibit REE patterns (with the exception of Eu) that are generally subparallel to parallel to basalt and diabase patterns (e.g., Hole $921 \mathrm{~B}$ and $921 \mathrm{C}$ diabase). Pyroxene-rich samples tend to exhibit lower LREE/HREE ratios than pyroxene-poor samples, as expected from partition coefficients for clinopyroxene, because plagioclase will always dominate the REE signature of olivine in clinopyroxene poor rocks (Fig. 20). Overall REE abundances in samples from Site 921 show large variations from less than $1 \times$ chondrite to $90 \times$ chondrite.

Several additional trace-element anomalies are obvious in the trace-element patterns in addition to Eu anomalies. The fractionation of MARK MORB generally demonstrates that the bulk distribution coefficient for $\mathrm{Sr}$ is $\sim 1$ throughout a large intervals of fractionation. The concentrations of $\mathrm{Sr}$ in basalts is nearly constant, but increasingly negative anomalies develop as basalts become more evolved and REEs become enriched relative to $\mathrm{Sr}$. The cumulates from Leg 153

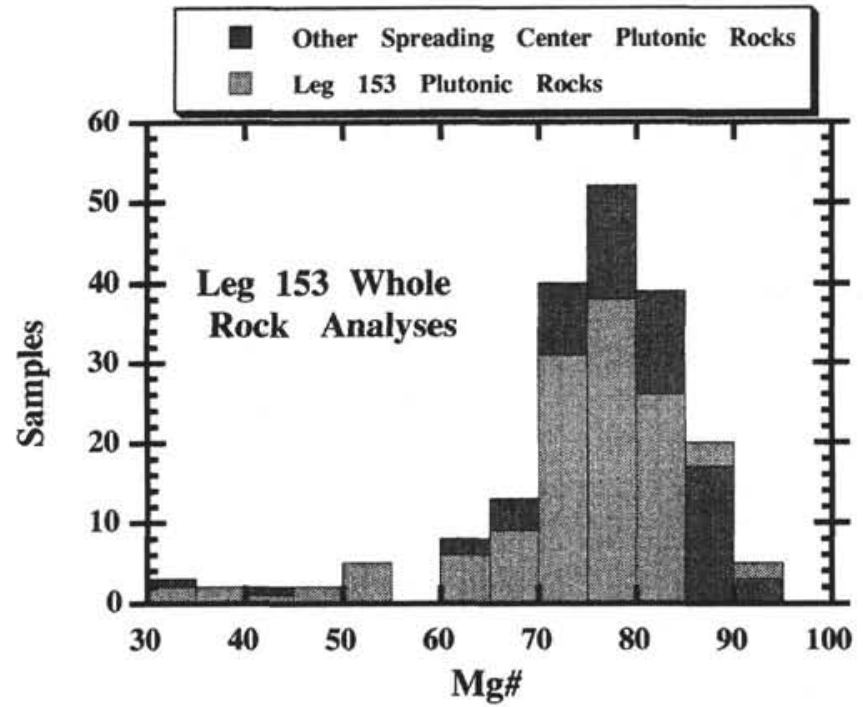

Figure 18. Histogram showing a cumulative comparison of plutonic recoveries in Leg 153 with those of other spreading centers as a function of Mg\#s (data sources listed in text). The Leg 153 plutonic samples span the entire range of these previously collected oceanic suites.
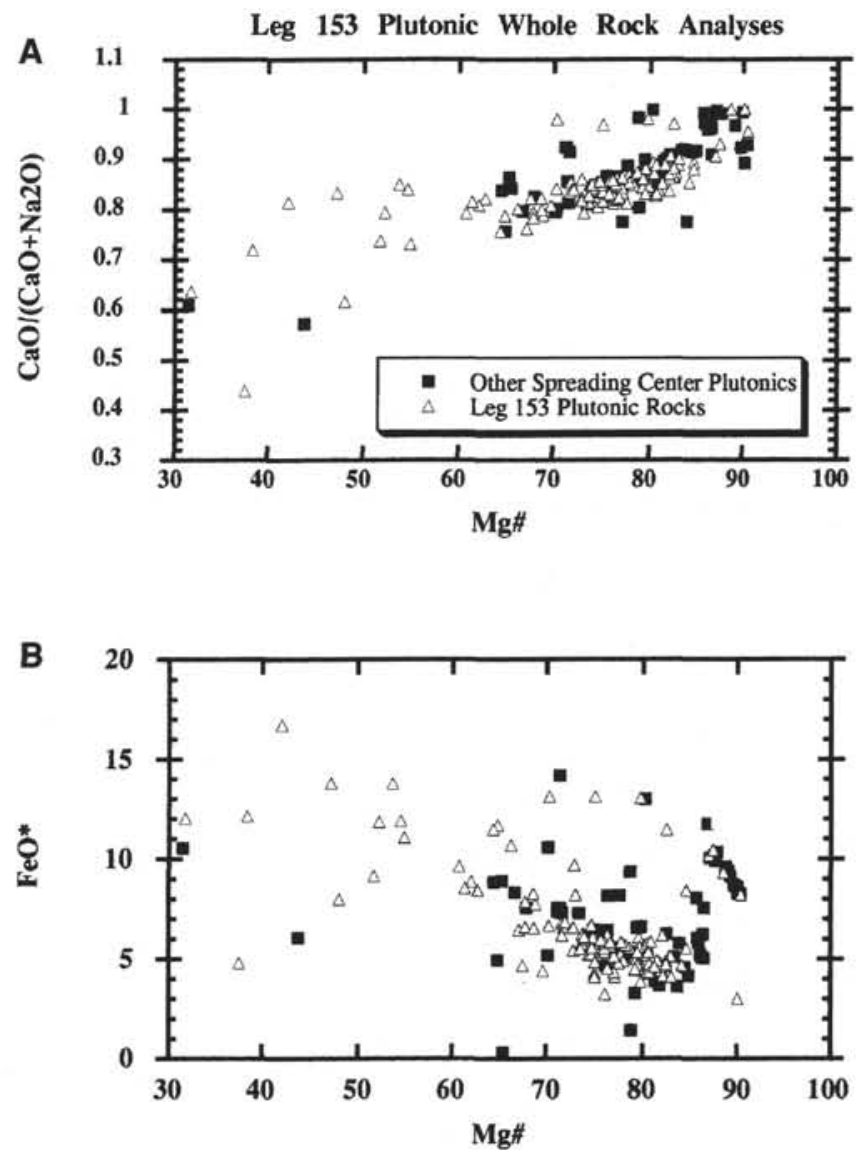

Figure 19. Chemical comparison of Leg 153 gabbroic samples with those recovered from other spreading centers: $\mathbf{A} . \mathrm{CaO} /\left(\mathrm{CaO}+\mathrm{Na}_{2} \mathrm{O}\right)$ vs. $\mathrm{Mg} \#$. B. $\mathrm{FeO}$ vs. $\mathrm{Mg} \#$ (data sources as above). 

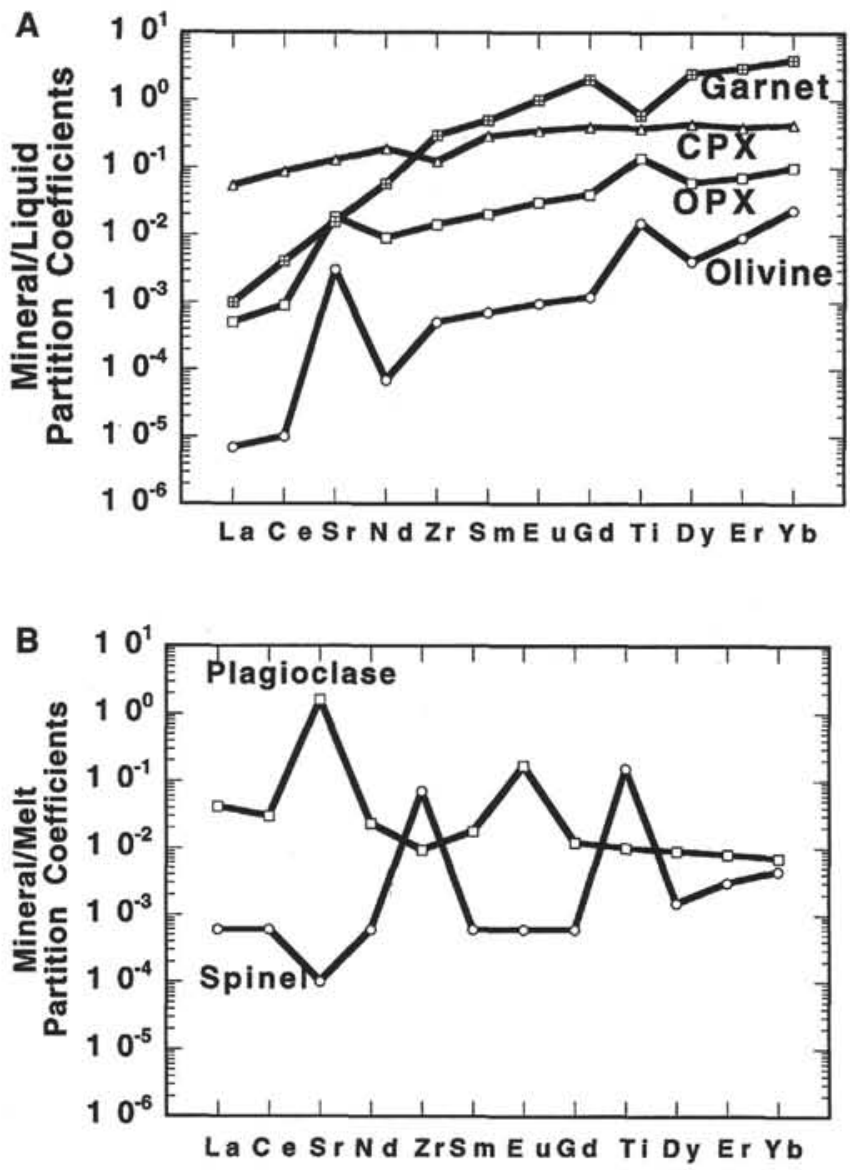

Figure 20. Mineral/liquid partition coefficients for primary mantle assemblages. A. Olivine, orthopyroxene (OPX), clinopyroxene (CPX), and garnet B. Plagioclase and spinel (references listed in Table 6). Note the sharp contrast between plagioclase and clinopyroxene, which control the overall abundances within the plutonic rocks.

show complementary large positive anomalies $(\mathrm{Sr} * / \mathrm{Sr}=1$ to 6$)$, but with average $\mathrm{Sr}$ abundances closer to basalts and diabases, as expected. The scatter in $\mathrm{Sr}$ abundances at a given $\mathrm{Mg} \#$ and modal plagioclase abundance can, however, be substantial. In Holes 921B and $921 \mathrm{C}$, average $\mathrm{Sr}$ abundances in gabbros are nearly identical to the abundances in diabases, suggesting that the diabases and gabbros may have similar parents. Negative $\mathrm{Sr}$ anomalies were observed, but only in the most highly fractionated oxide gabbros and felsic samples (Figs. 22, 23) where REEs are very enriched. Small positive $\mathrm{Zr}$ anomalies are also present in many of the least fractionated samples, and these are also present in many diabase and basalts from the MARK regions (Bryan et al., 1994; J.F. Casey, W. Bryan, and G. Thompson, unpubl. data; also see Kempton and Casey, this volume). These small $\mathrm{Zr}$ anomalies are interpreted to reflect the original composition of parental MORB melts, and is a consequence of the relatively low degrees of spinel facies melting (see below) that characterize MARK basalts. Large positive anomalies are present in oxide-rich gabbros and felsic samples (with $\mathrm{Zr} * / \mathrm{Zr}$ up to 11 in Sample 921E-8R-1, 91$95 \mathrm{~cm}$ ). As in the case of Site 921 gabbroic rocks (Figs. 22, 23), these anomalies reflect accumulation of zircon when strongly positive. Weak negative $\mathrm{Ti}$ anomalies are present in most of the olivine gabbros and have also been observed in MARK basalts and diabase. Likewise, these weak anomalies can be derived by low degrees of spinel facies melting of a depleted fertile MORB mantle source (see below). Small positive $\mathrm{Zr}$ and small negative $\mathrm{Ti}$ anomalies are a common feature in MARK basalts and are characteristic of primitive gabbroic rocks. Ti anomalies can become positive in oxide gabbros be- cause of accumulation of $\mathrm{Fe}-\mathrm{Ti}$ oxides or negative when extensive Fe-Ti oxides have fractionated from the melt in equilibrium with gabbroic mineralogy. More felsic derivatives $\left(54 \%\right.$ to $\left.61 \% \mathrm{SiO}_{2}\right)$ such as Sample 921E-5R1, 45-51 cm, show strong enrichment in the REE and negative $\mathrm{Ti}$ and $\mathrm{Sr}$ anomalies, reflecting very evolved stages of fractionation (Fig. 23). Even though the $\mathrm{Sr}$ anomalies are strongly negative in these samples, the $\mathrm{Sr}$ abundances are similar to their less fractionated counterparts, again reflecting the fact that the bulk distrihution coefficient, D, must approximate one throughout the fractionation history. The anomaly changes from positive in primitive samples to negative in evolved samples because increased fractionation steadily increases other inconipatible elements relative to a nearly constant $\mathrm{Sr}$ abundance. These more fractionated nelts have similarities reminiscent of the enriched REE patterns and large negative $\mathrm{Sr}$ and $\mathrm{Ti}$ anomalies previously described for some pyroxenites found with Holes 920B and 920D. The main differences are that the bulk-rock Mg\#s for pyroxenites are in the range from 80 to 90 in Holes 920B and 920D, whereas the felsic samples have bulk Mg\#s that are very low $(30-50)$.

\section{Site 922}

Samples from Holes 922A and 922B show the strongest contrast and diversity in compositions. Rock types vary from relatively primitive troctolites to oxide gabbros. Sample bulk chemistries (see Table 4) show that the wt\% FeO* content varies between 4.67-16.71 and bulk-rock Mg\#s vary between 42 and 84 over relatively short distances (several m). $\mathrm{SiO}_{2}$ is typically depleted in oxide gabbros, and $\mathrm{P}_{2} \mathrm{O}_{5}$ can reach up to $3.35 \mathrm{wt} \%$, reflecting apatite accumulation. Oxide gabbros are present in both holes in a narrow interval between 5 and $17 \mathrm{~m}$ depth. The downhole plots of $\mathrm{Mg} \#$ and Ca\# (Fig. 24) show that fractionated gabbros are intimately mixed on a small scale with relatively primitive gabbroic rocks. Trace-element patterns show very strong enrichments in the oxide gabbros with REE abundances in excess of $100 \times$ chondrite in some samples (Fig. 25). In part, the high abundances reflect the accumulation of accessory phases rich in REEs such as apatite (e.g., Sample 922A-3R-1, 104-109 cm), although these evolved samples tend not to have significantly fractionated LREE relative to HREE. LREE/HREE ratios lie close to typical basalt ratios $(0.5-1.2)$. Oxide gabbros generally show pronounced negative $\mathrm{Sr}, \mathrm{Zr}$, and $\mathrm{Ti}$ anomalies, reflecting the fractionated nature of the melt from which they solidified. In oxide gabbros, depletion in $\mathrm{Zr}$ and $\mathrm{Ti}$ can be explained by fractionation of zircon and $\mathrm{Fe}-\mathrm{Ti}$ oxides, respectively. Strong negative $\mathrm{Sr}$ anomalies will also develop in the melt because of steady increases in REE abundances and near constant $\mathrm{Sr}$ abundances during fractionation. The oxide-rich gabbro samples also tend to have elevated abundances of $\mathrm{V}$ because of oxide accumulation. Less fractionated samples tend to have strong positive $\mathrm{Sr}$ and weak positive $\mathrm{Zr}$ anomalies and negative $\mathrm{Ti}$ anomalies, as observed at Site 921 . All samples from these holes generally have somewhat more elevated abundances of incompatible elements for a given $\mathrm{Mg} \#$ when compared with samples from Sites 921 and 923 , suggesting that high fractions of trapped melt may be involved in the section. Troctolitic gabbros tend to have LREE-enriched patterns and more elevated abundances than troctolites from Sites 921 and 923.

\section{Site 923}

Hole $923 \mathrm{~A}$ is generally characterized by a significantly smaller range of bulk-rock compositions than holes at either Site 921 or 922 gabbroic and felsic rocks, yet it represents the hole that yielded the most continuous gabbroic core recovery of any hole drilled during Leg 153 (Fig. 2B). Therefore, we present the downhole mineral chemistry for this hole because interpretations are best constrained here. Bulk-rock Mg\#s range from $\sim 38$ to 85 , but with the exception of one oxide gabbro, all other samples lie between Mg\#s of 65 and 85 . $\mathrm{FeO} *$ ranges from 4.2 to $14.2 \mathrm{wt} \%$, but likewise $\mathrm{FeO} *$ generally 

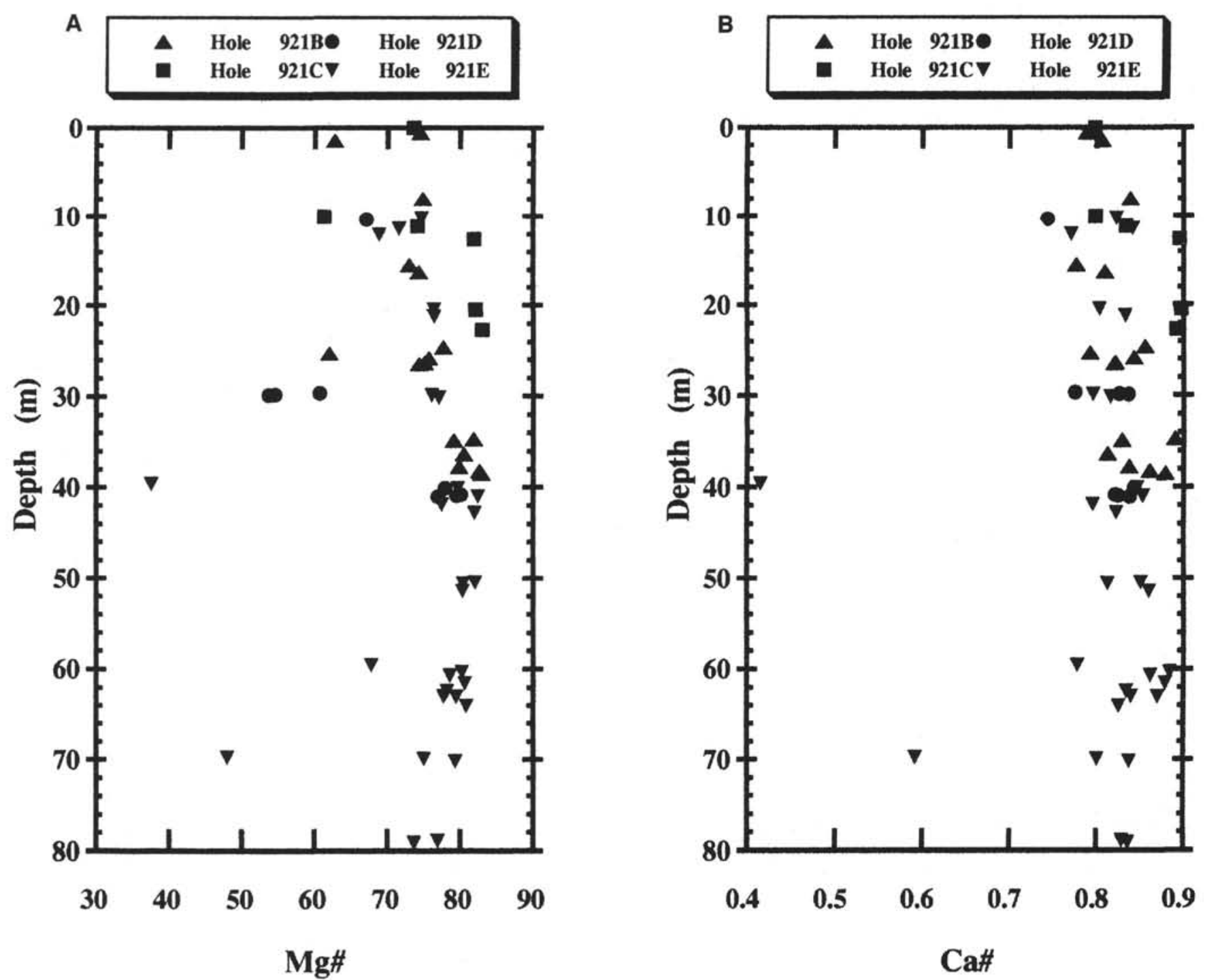

Figure 21. A. Downhole variations of $\mathrm{Mg \# s}$ for gabbroic samples in Holes 921B -921E. B. Downhole variations of Ca\#s in Holes 921B-921E.

has a more limited range. Downhole plots of bulk-rock Mg\# and Ca\# (Fig. 26) show that most samples lie within a small compositional range and that the oxide gabbro is somewhat anomalous, again showing a large compositional gap. Figure 26 is scaled to eliminate the oxide gabbro and shows the correlation between forsterite (Fo) content of olivine and $\mathrm{Mg} \#$ of clinopyroxene relative to the bulk-rock $\mathrm{Mg \#}$. At least five major and abrupt changes in the trends of $\mathrm{Mg \# s}$ have been marked, and these cryptic chemical variation are thought to represent variations brought about by either distinct injections of more primitive magma (e.g., 30, 47, and $68 \mathrm{mbsf}$ ) or more evolved magma ( 22 and $62 \mathrm{mbsf}$ ). There are also more gradual changes in magma compositions because of mixing with either more primitive or more fractionated magmas between these abrupt changes. Shipboard descriptions of the Hole 923A core (Cannat, Karson, Miller, et al., 1995) showed downhole mineralogic changes (modal layering) that imply cryptic chemical variations. These are characterized by repeated cycles of olivine-rich troctolite, olivine gabbro, and gabbro (also shown in Fig. 26). The modal changes observed involved higher resolution observations when compared with the more limited number of samples involved in this geochemical study. Several cycles were noted, with major changes in modal mineralogy at $22,30,47,54,62$, and $68 \mathrm{mbsf}$ and are identical to major changes in mineral and bulkrock $\mathrm{Mg} \# \mathrm{~s}$. These are characterized by major changes in modal olivine/clinopyroxene ratios.
Inverse trends (increasing $\mathrm{Mg \# s}$ upward) appear to be as common as normal fractionation trends (decreasing Mg\#s upward). The thickness of cryptic units marked by these major changes ranges from a few meters to $\sim 20 \mathrm{~m}$. These scales of cryptic chemical variation and modal variation are similar to those observed by similarly detailed high-resolution sampling studies of the plutonic sections of gabbroic rocks in ophiolites (e.g., Elthon et al., 1984a; Komor et al., 1985a, 1987). Both Mg\#s of clinopyroxene and An of plagioclase in Hole 923A show significant ranges in core-to-rim studies in many of the samples, demonstrating that cumulates have probably been affected by crystallization of trapped or migrant melt within the cumulate pile. Some of the plagioclase variation is thought to be the result of alteration (Agar et al., this volume). Olivine tends to be more homogeneous in composition, apparently because it reequilibrates more rapidly with interstitial melt (e.g., Hess, 1992).

Trace-element data for Hole 923A show a much narrower range in abundances when compared to data from either Site 921 or 922 (see Fig. 27). One oxide gabbro sample (Sample 923A-4R-1, 3-10 $\mathrm{cm}$ ) shows the most unusual pattern in being characterized by very strong positive $\mathrm{Ti}, \mathrm{V}, \mathrm{Sr}$, and $\mathrm{Zr}$ anomalies, but low overall $\mathrm{REE}$ abundances of less than $10 \times$ chondrite, unlike oxide gabbros from Sites 921 and 922 that show more elevated abundances. Sample 923A-7R-2, 1-7 cm, also has an unusual chemistry in being characterized by relatively high abundances of REEs and high $\mathrm{La} / \mathrm{Yb}$ ratios, 

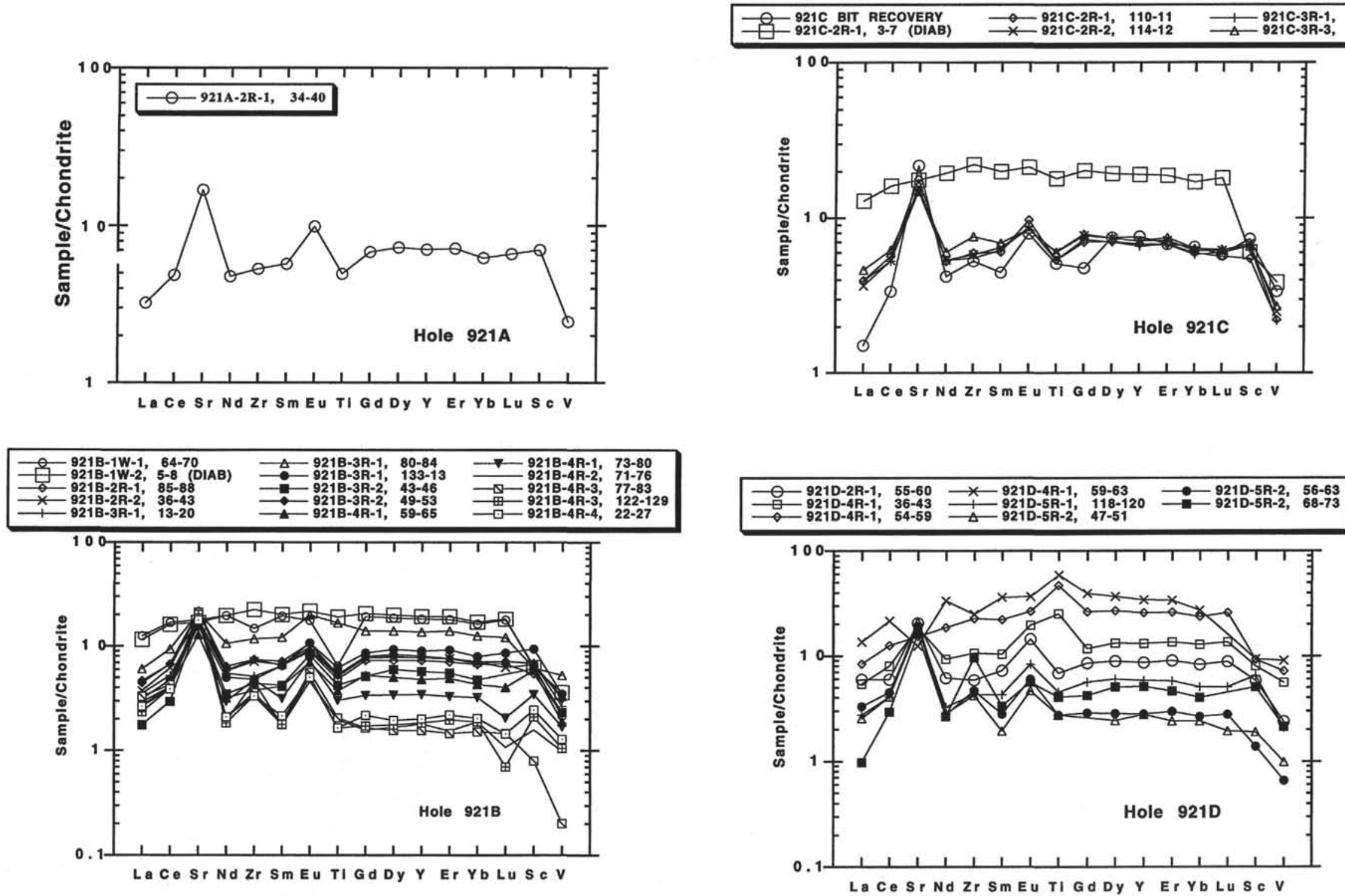

Figure 22. Chondrite-normalized trace-element spidergrams for Holes 921A-921D. The plotted trace elements are arranged in the order of increasing compatibility toward the right-hand side. All samples are gabbroic rocks except diabases (DIAB) that cut gabbroic rocks in Holes 921B and 921C. Note that $\mathrm{Sr}$ approximates the same value for diabases and gabbroic rocks, indicating similar parents and an average bulk $\mathrm{D}$, that is $\sim 1$ for $\mathrm{Sr}$. 
Figure 23. Chondrite-normalized trace-element spidergrams for Hole 921E. Samples are listed in order of the depth of recovery and all lithologies are included in Table 3 for reference. Note that diorite and some felsic samples show very high abundances of trace elements, whereas troctolites show very low abundances of trace elements and LREE/HREE enrichments. Note also that felsic rocks and oxide gabbros have negative $\mathrm{Sr}$ and $\mathrm{Ti}$ anomalies. The oxide gabbro shows a strong positive $\mathrm{Zr}$ anomaly.
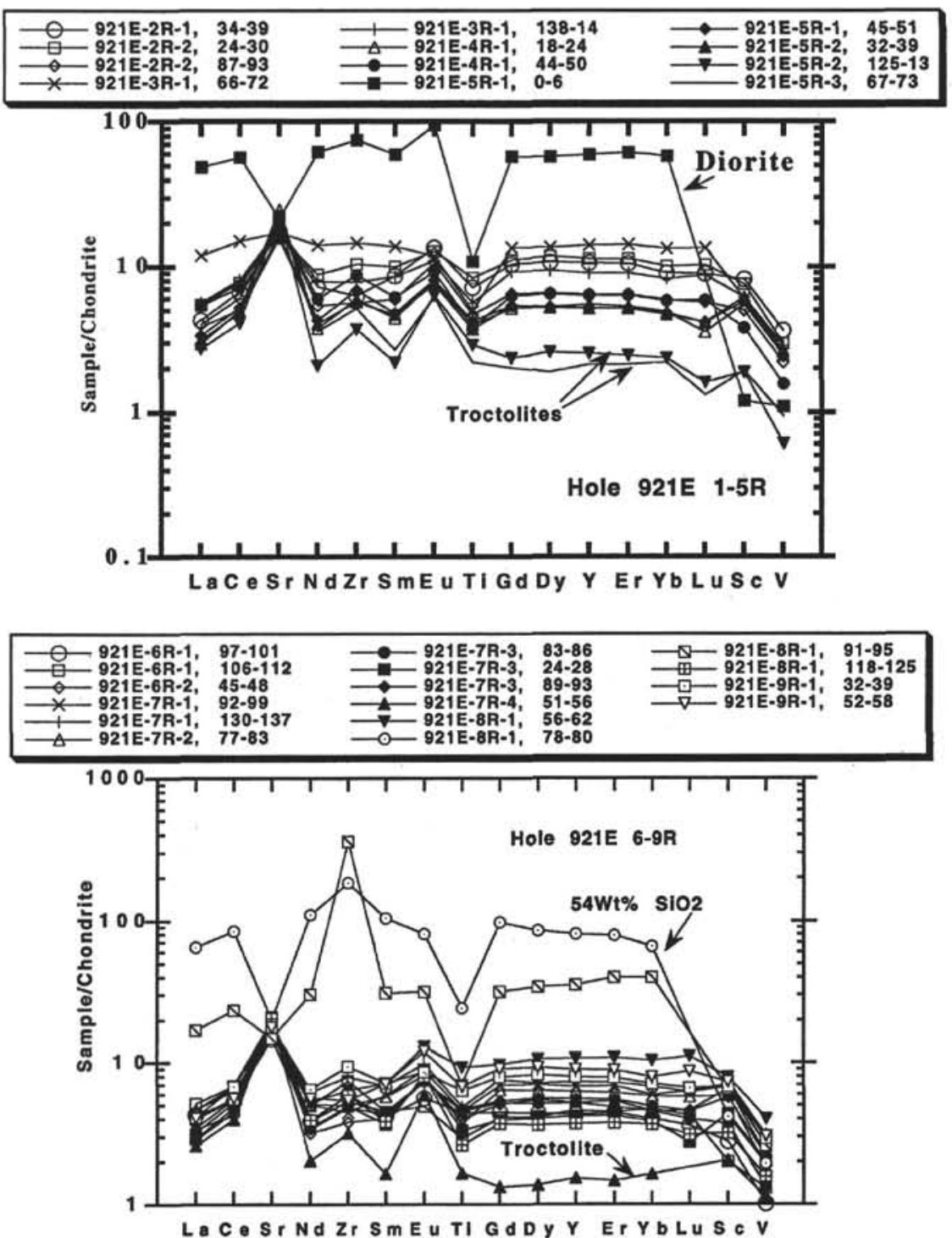

yet it is more primitive than most gabbros with elevated abundances. Sample 923A-15R-2, 0-6 cm, shows similar abundances and a distinct positive $\mathrm{Zr}$ anomaly, but is significantly more iron rich and fractionated. Other characteristics of the trace-element patterns for the more primitive samples are similar to those previously described for Site 921 and 922 . Patterns are characterized by positive $\mathrm{Sr}, \mathrm{Zr}$, and Eu anomalies, and slight negative Ti anomalies.

\section{PRIMITIVE AND EVOLVED LEG 153 DIABASE SAMPLES (HOLES 920B, 920D, 921B, AND 921C)}

In addition to gabbroic and ultramafic samples recovered during Leg 153, several diabase samples were recovered. These diabases exhibit well-defined intrusive contacts and relationships with peridotites in Holes 920B and 920D and with gabbroic rocks in Holes 921B and 921D (Cannat, Karson, Miller, et al., 1995). The samples of diabase analyzed here are reported in the respective bulk-rock tables for each site and discussed briefly below. Their major-element, trace-element, and isotope geochemistry is more fully evaluated in Kempton and Casey (this volume).

Diabase samples from the gabbroic holes (Holes 921B and 921C) appear more evolved and very similar to MARK basalts in terms of
$\mathrm{MgO}, \mathrm{Na}_{2} \mathrm{O}$, and trace-element abundances (Bryan et al., 1981, 1994; J.F. Casey, W. Bryan, and G. Thompson, unpubl. data). Although altered, they are characterized by relatively low LOI (1.41-1.93) when compared with their more altered diabase counterparts from Holes 920B and 920D. Extended trace-element patterns for Hole 921B and $921 \mathrm{C}$ diabases lack any evidence of alteration, such as the positive $\mathrm{Sr}$ and $\mathrm{La}$ anomalies observed in Hole 920B and 920D diabases. Likewise, Site 921 diabases show ${ }^{87} \mathrm{Sr} /{ }^{86} \mathrm{Sr}$ ratios similar to unaltered MARK basalts (Kempton and Casey, this volume).

Samples of diabases intruding ultramafic rocks in Holes 920B and 920D, however, are noteworthy because of their apparent primitive nature. They are characterized by high $\mathrm{MgO}$ (12.14 to $15.08 \mathrm{wt} \%)$, Mg\#s ( 73.6 to 75.7 ), Ni contents (228 to $280 \mathrm{ppm}$ ), and $\mathrm{Cr}$ contents between (391 and $489 \mathrm{ppm}$ ). Mg\#s for these diabases are similar to equilibrium melts derived from melting of MPY-87 (Green and Falloon, 1987) and to those generated in the sandwich experiments of Fujii and Scarfe (1985). These values are certainly in the range to be in Fe-Mg exchange equilibrium with harzburgitic olivine $(\mathrm{Mg} \#=91-$ 92). It is therefore tempting to speculate that these diabases represent near-primary melts or parental MARK melts, which may have been in equilibrium with the mantle. It is important to recognize, however, that even such primitive magma types intruding mantle rocks are probably not in equilibrium with the mantle at any pressure, but rep- 


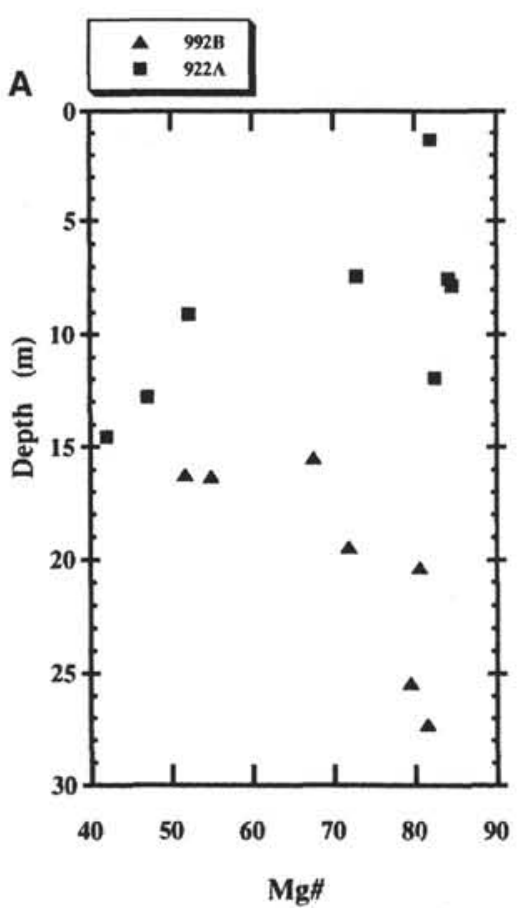

resent mixtures of primary magmas generated within the melting column at a variety of depths (Klein and Langmuir, 1987). Although two of the high-MgO basalts were taken near chill margins to avoid accumulation effects and the petrography of the samples would not indicate extensive phenocryst accumulation, the samples are highly altered and should probably not be considered liquid compositions.

This is a troubling aspect of the samples and the proposal that they represent near-primary or parental melt. Extensive alteration is indicated by the high volatile abundances ( $>5 \mathrm{wt} \%$ ), high LOI (Tables 1 , 2 ), and what appears to be an obvious mobile-element anomaly in $\mathrm{Sr}$ content in all Site 920 diabases (Fig. 27). Perfect fractional crystallization modeling of the diabase composition shows that olivine, clinopyroxene, and plagioclase would crystallize on the liquidus, unlike typical melts in equilibrium with mantle assemblages in which olivine is likely to be the sole phase on the liquidus at low pressures. $\mathrm{CaO}$ is exceptionally low in these diabases and appears to have been depleted by alteration. Although $\mathrm{Na}_{2} \mathrm{O}$ and $\mathrm{K}_{2} \mathrm{O}$ do not appear enriched in the diabase samples with respect to other MARK basalts, the extended trace-element diagram (Fig. 27) indicates that each of the diabases from Site 920 peridotite holes is characterized by a strong positive $\mathrm{Sr}$ anomaly ( $\mathrm{Sr} * / \mathrm{Sr}$ up to 2.94 ). It is important to note that magma fractionation effects generally produce negative $\mathrm{Sr}$ anomalies within melts. Accumulation of plagioclase could result in positive anomalies, but this appears unlikely because the diabases from quenched dike margins do not appear to have abundant plagioclase phenocrysts. We conclude that the positive anomaly appears to be an alteration effect. Additional evidence of $\mathrm{Sr}$ mobility includes the high ${ }^{87} \mathrm{Sr} /{ }^{86} \mathrm{Sr}$ ratio relative to unaltered MARK basalts (Kempton and $\mathrm{Ca}-$ sey, this volume). One of the samples, Sample 920D-8R-3, 66-73 $\mathrm{cm}$, also shows a high-La spike that departs from the usually smooth chondrite-normalized REE pattern. This could also result from extensive alteration of the diabase if high water/rock ratios were involved. Similar mobilization of La in altered pillow lava rims and zeolite and lower greenschist facies basalts is discussed by Ludden and Thompson (1979), Siefert et al. (1987), Menzies et al. (1977), and Humphris (1984). Chlorite is a significant phase replacing olivine in these samples and significant chlorite formation during alteration can result in increases in bulk $\mathrm{MgO}$ and depletion of $\mathrm{CaO}$ during alteration (see Kempton and Casey, this volume). This may, in part, explain the high $\mathrm{MgO}$ content and low $\mathrm{CaO}$ content of these diabases. In addition to the potential of raising the bulk $\mathrm{MgO}$ content of the diabase, chlorite formation has been noted to result in preferential uptake of La (Menzies et al., 1977). This could possibly explain the positive spike in $\mathrm{La}$ in diabase Sample 920D-8R-3, 66-73 cm. Ce appears little affected, probably because the samples were not within the seafloor weathering zone where positive $\mathrm{Ce}$ anomalies commonly develop (Ludden and Thompson, 1979).

Modeling of the prealteration $\mathrm{MgO}$ content of these samples indicates that the diabases contained approximately $\sim 11 \mathrm{wt} \% \mathrm{MgO}$ (Kempton and Casey, this volume), which is still far more primitive than MARK basaltic compositions (Bryan et al., 1994). The fact that compatible elements $\mathrm{Ni}$ and $\mathrm{Cr}$ contents, which are considered relatively immobile during alteration, are approximately twice as high as the most primitive MARK basalts with ( $8-9 \mathrm{wt} \% \mathrm{MgO}$ ) reinforces the notion that these diabases were, in fact, very primitive in comparison to other MARK basalts before alteration (Bryan et al., 1981, 1994; J.F. Casey, W. Bryan, and G. Thompson, unpubl. data). The immobile trace-element patterns of the Site 920 diabases lie parallel to MARK basalt patterns, indicating they are probably nearly cogenetic and perhaps parental to MARK MORB. With the exception of $\mathrm{La}$ and $\mathrm{Sr}$, other segments of the trace-element patterns for Hole 920B and 920D diabases consist of relatively immobile REEs and HFSEs (Fig. 28) that lie near the lower portion of the field of unaltered MARK basalts (J.F. Casey, W. Bryan, and G. Thompson unpubl. data). Thus, with the exception of $\mathrm{Sr}$ and in one case $\mathrm{La}$, these patterns appear to be little affected by metasomatism. They lie at the low abundance or least fractionated edge of the MARK basalt field, consistent with their high $\mathrm{Ni}$ and $\mathrm{Cr}$ abundances. One notable feature of the diabase samples from Sites 920 and 921 is the small positive $\mathrm{Zr}$ anomaly, a feature that is also typical of MARK basalts and many of the trace-element patterns for Leg 153 gabbroic samples.

Because these diabases appear to be the most primitive yet recognized from the MARK region, we have utilized immobile, extended trace-element patterns to model the average extent of partial melting in the MARK region. Partial melting of the mantle at mid-ocean ridges results from decompression of the upwelling mantle as it ascends along the adiabat and crosses the mantle solidus. Here we adopt the findings of Johnson et al. (1990) that melting of the mantle by a batch melting process cannot explain the trace-element abundances of residual clinopyroxene and that melting must be near fractional. The 


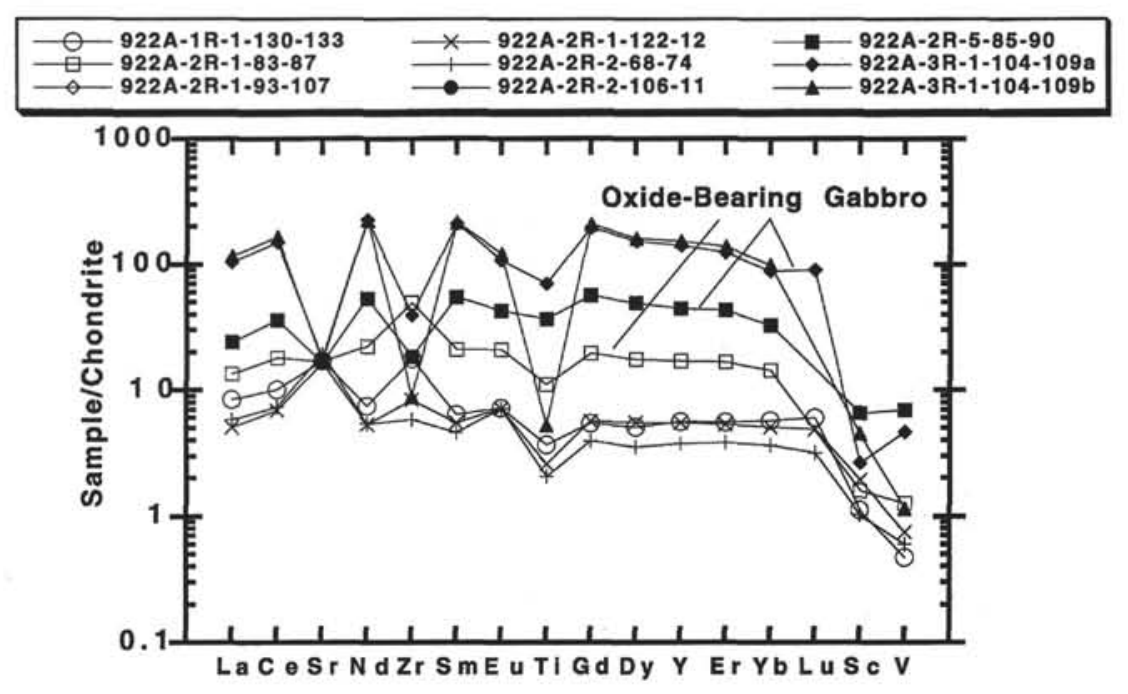

Figure 25. Chondrite-normalized trace-element spidergrams for Holes $922 \mathrm{~A}$ and $922 \mathrm{~B}$. Notice the highly elevated trace-element abundances in oxide-bearing gabbros. Lithologies and data are included in Table 4.

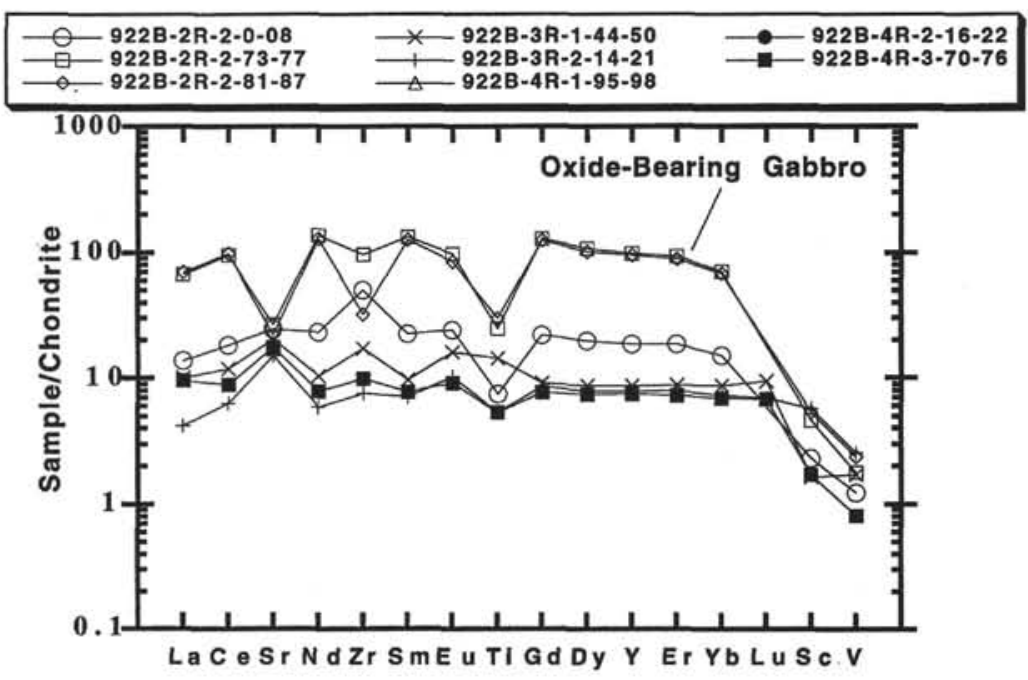

depth of initial melting will depend on the composition of the peridotite, the temperature of the adiabat, and the dynamics of the melting process (McKenzie, 1984; Klein and Langmuir, 1987; McKenzie and Bickle, 1988). In the model of Klein and Langmuir (1987), a range of near-fractional melt compositions are produced within a polybaric melting column. These melts pool to form MORB and their overall geochemical characteristics reflect the nature of the melting regime (temperature, pressure, fluid content, and mantle source composition).

To evaluate the extent of melting, we have utilized a continuous (near-fractional) melting model with $1 \%$ residual mantle porosity (McKenzie, 1985; McKenzie and Bickle, 1988) and a depleted MORB mantle source similar to that utilized by Johnson et al. (1990). The patterns of near-fractional and linearly mixed pooled melts generated by these models at Fmax of $0.05 \%, 5 \%, 10 \%, 15 \%, 20 \%$, and $25 \%$ partial melting are displayed in Figure 29 . Kds used in the model are listed in Table 6. The equations of Sobolev and Shimizu (1992) for continuous melting were used in the calculations. Melting models for both garnet and spinel facies melting were also tested along with models using strictly spinel facies melting, but it was clear that even small amounts of early garnet melting would produce pooled traceelement patterns too depleted in HREE to fit the MARK patterns. Thus, all patterns shown are for melting in the spinel facies. The characteristics of the two sets of melting patterns are that the near-fractional melts are rapidly depleted in highly incompatible elements when compared with the pooled melts at similar fractions of melting.
Pooled melts are similar to melts generated by batch equilibrium melting models (Langmuir et al., 1992). There is significantly less variation and lower extents of incompatible-element depletion within the pooled melt over the same range of melting. The MARK basalts show near-parallel patterns to pooled melt compositions generated by the model. The only basalts in the MARK region that are nonparallel have slightly depleted HREE and chondrite-normalized $\mathrm{Ce} / \mathrm{Yb}$ ratios greater than one. They are from the more LREE-enriched region of the Mid-Atlantic Ridge, between $22^{\circ}$ and $23^{\circ} \mathrm{N}$ and a zone of high-Al basalts. This depletion in HREE can be explained possibly by plagioclase accumulation, or assimilation in high- $\mathrm{Al}$ basalts, or different mantle source characteristics (Bryan et al., 1994).

Melting in the spinel field successfully duplicates trace-element patterns for Site 920 and Site 921 diabases (i.e., they lie nearly parallel to the pooled melt patterns generated by the model; see pooled melts patterns in Fig. 27). Because the Hole 920D and 920B diabase samples are the most primitive basaltic compositions sampled in the MARK area and they are unlikely to be significantly fractionated, the estimate of the average extent of melting is based on these samples. Site 921 diabases, which are more fractionated, are shown only for comparison. Based on this modeling, the highest extent of melting derived from Site 920 diabases represents an Fmax of approximately $12 \%-15 \%$ (Fig. 28), which is consistent with predictions made on the basis of major-element glass compositions (see above). A more detailed look at the comparison of models shows that, although the middle to heavy REEs match closely with the $12 \%-15 \%$ melting models, 


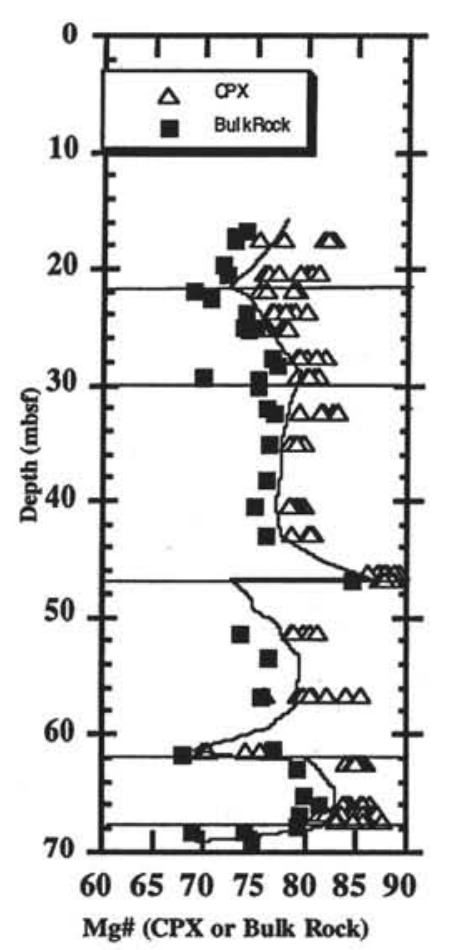

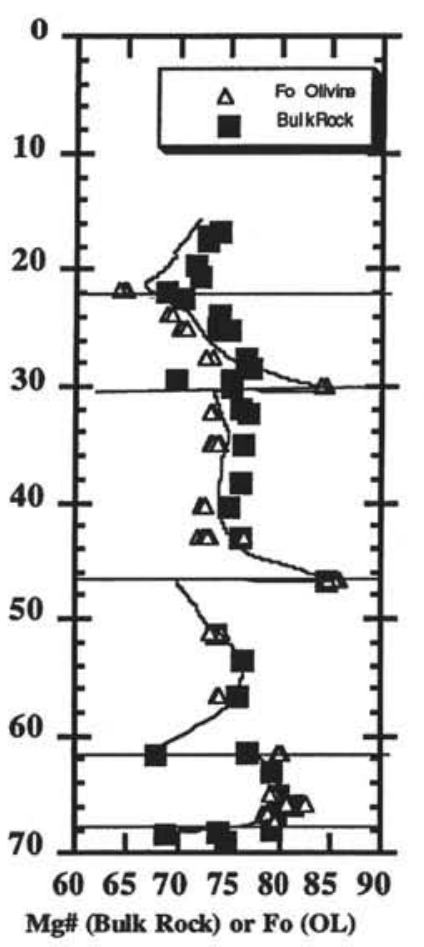

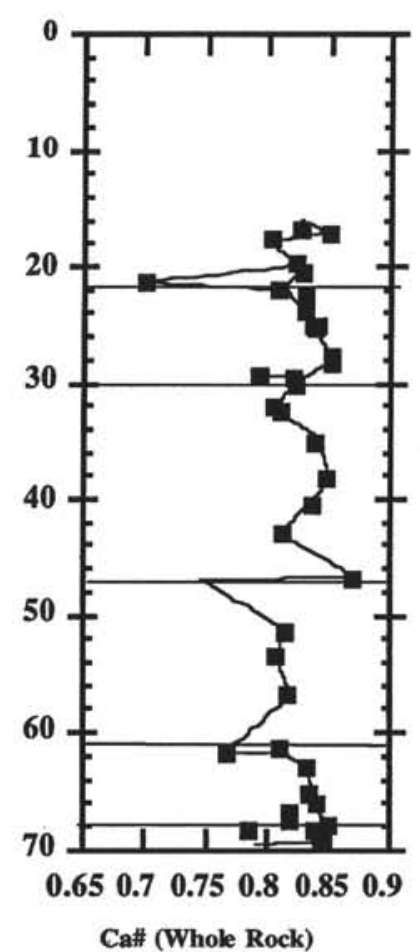

Figure 26. Downhole variation plots (from left to right) of bulk-rock Mg\# + clinopyroxene (CPX) Mg\#, bulk-rock Mg\# + olivine forsterite, bulk-rock Ca\# for Hole 923A, plagioclase anorthite content, and shipboard modal olivine variation showing cycles of olivine + plagioclase, olivine + plagioclase + clinopyroxene crystallization (shaded triangles show a schematic with number of cycles noted from modal data). Cryptic chemical variation described by the whole-rock and mineral chemistry data are consistent with periodic recharge of the magma chamber, magma mixing, and fractional crystallization. There is a correlation between large modal changes and chemical changes. Note that normal fractionation trends (decreasing Mg\#) are as common as inverse changes. Horizontal lines mark regions of the core where abrupt modal and/or chemical changes occur in one or more diagrams. 

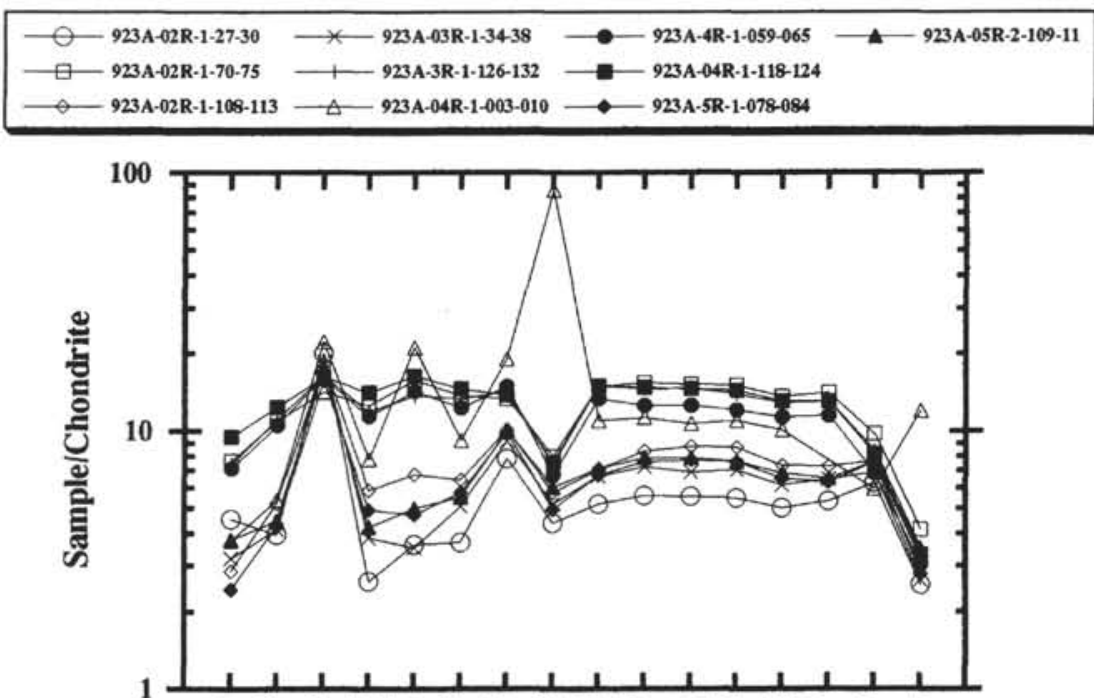

La Ce Sr NdZr SmEu Ti GdDy Y Er Yb Lu Sc V

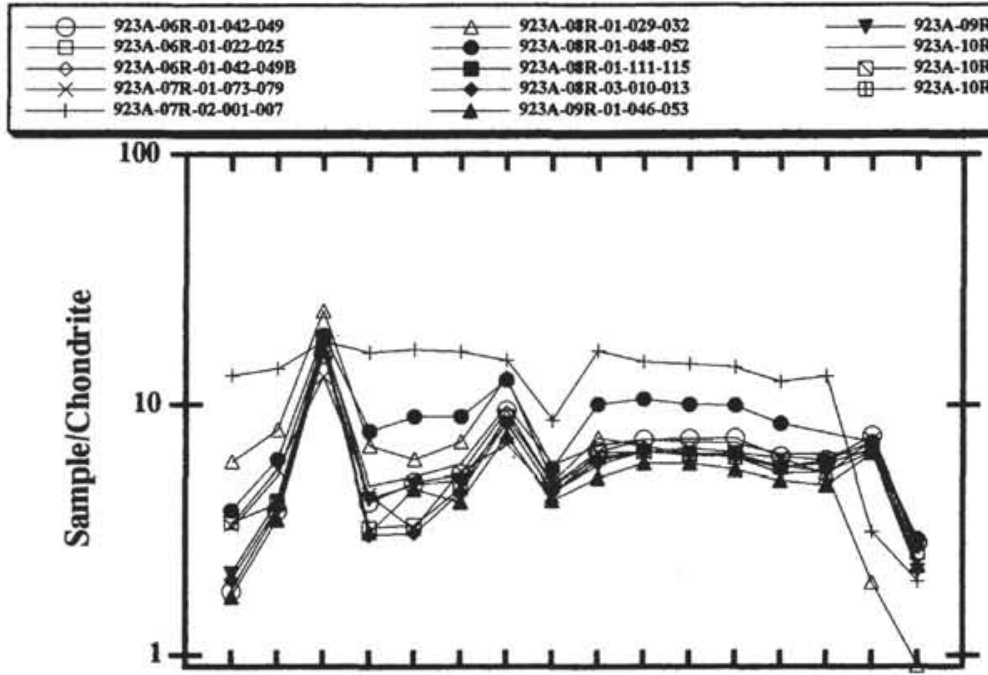

La Ce Sr NdZr SmEu Ti GdDy Y Er YbLu Sc V
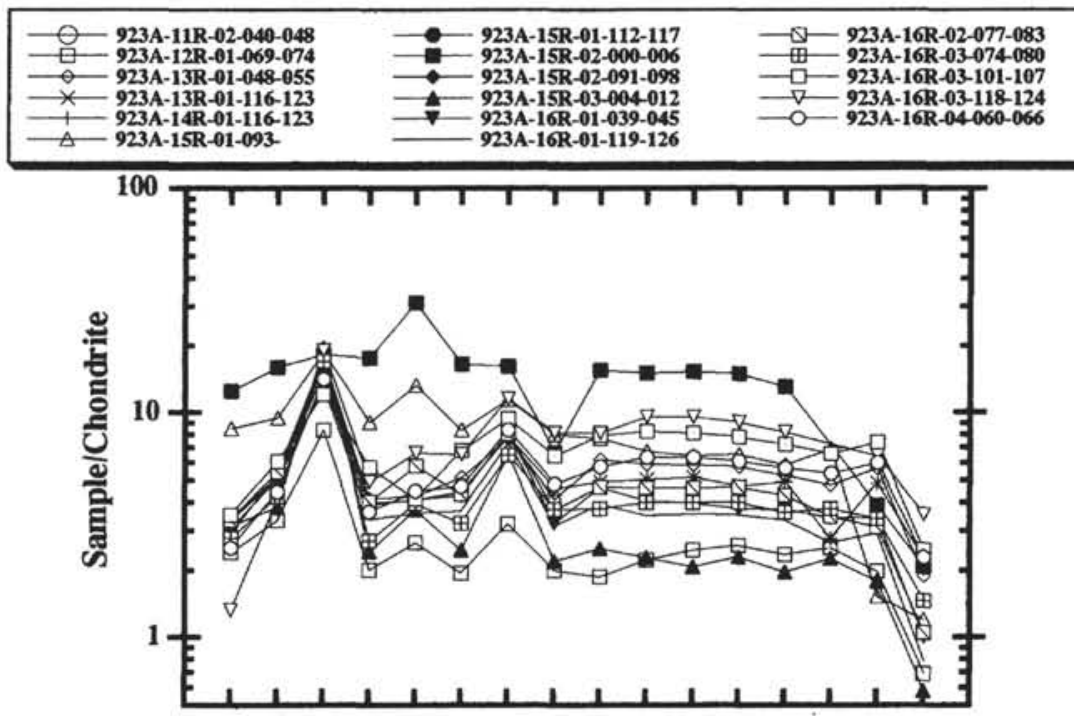

La Ce Sr NdZrSmEu Ti GdDy Y Er YbLu Sc V

Figure 27. Chondrite-normalized trace-element spidergrams for Hole 923A samples. Lithologies and data are included in Table 5. 


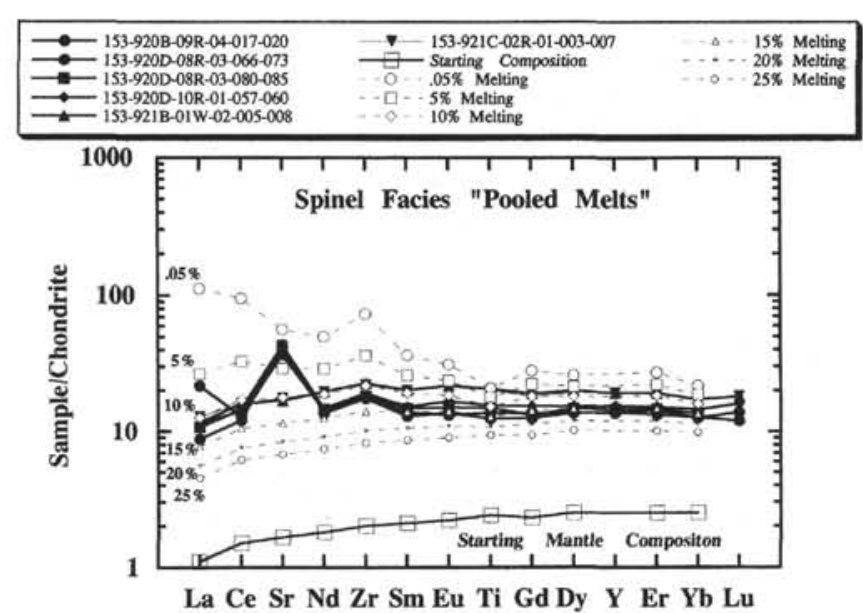

Figure 28. Extended REE spidergram for the diabase samples recovered in Holes 920B, 920D, 921B, and 921C. Notice that samples from Hole 920B and $920 \mathrm{D}$ are characterized by a positive $\mathrm{Sr}$ spike that appears to be an indication of strong hydrothermal alteration. Other immobile elements and HSFEs (e.g., $\mathrm{Zr}$ and $\mathrm{Ti}$ ) do not show signs of alteration. With the exception of La in Sample 920B-9R-4, 17-20 cm, the immobile elements (REEs and HSFEs, such as $\mathrm{Zr}$ and $\mathrm{Ti}$ ) do not show signs of alteration. Also shown are the results of a modeling for continuous melting for a depleted MORB manthe source with $1 \%$ residual mantle porosity. Melting was conducted in the spinel facies with $1 \%$ residual melt porosity in the mantle. The extended REE patterns for linearly pooled melts at $0.05 \%, 5 \%, 10 \%, 15 \%, 20 \%$, and $25 \%$ melting are shown for comparison with diabase analyses. The high$\mathrm{MgO}$ diabase samples recovered from Holes 920B and 920D indicate average melting of approximately $13 \%-15 \%$. Samples from Holes $921 \mathrm{~B}$ and 921C are considered significantly more fractionated than MARK parental magmas. Note the small positive $\mathrm{Zr}$ anomaly symptomatic of low extents of spinel facies melting. Source composition modeled is from Johnson and Dick (1992).

the LREEs can lie between $11 \%$ and $15 \%$. In part, it may indicate a slightly more LREE-enriched mantle source component in the MARK area than used in the modeling or possible mixing with a greater volume of more enriched components from deeper in the melting regime (Plank and Langmuir, 1992; Plank et al., 1995). There is some evidence of a more enriched mantle component in the MARK region based on isotopic results from Leg 153 gabbroic and MARK basaltic samples (Dosso et al., 1993; Kempton and Casey, this volume) and from REE data collected on basalts sampled between $22^{\circ}$ and $23^{\circ} \mathrm{N}$, which have chondrite-normalized $\mathrm{Ce} / \mathrm{Yb}$ ratios (Fig. 3) greater than 1 (J.F. Casey, W. Bryan, and G. Thompson, unpubl. data).

The similarity in trace-element patterns with the models generated includes distinctive, but small positive $\mathrm{Zr}$ anomalies of $\sim 1.26$ ), which is a characteristic of low extents of spinel facies melting in the model calculations. The fact that $\mathrm{Zr}$ anomalies are preserved is consistent with lower extents of melting as they tend to disappear in pooled melt compositions at higher extents of melting or when melting initiates in the garnet facies. These small $\mathrm{Zr}$ anomalies are also generally present in MARK basalts patterns (J.F. Casey, W. Bryan, and G. Thompson, unpubl. data), as well as the Leg 153 diabase and gabbroic trace-element patterns, reinforcing the evidence for low degrees of spinel facies melting $(F \max =12 \%-15 \%)$.

\section{COMPARISON OF THE BULK-ROCK TRACE ELEMENTS IN MARK PLUTONIC AND BASALTIC ROCKS}

To evaluate the trace-element abundances in plutonic rocks, a more complete understanding of the nature of the melt compositions generated in the subaxial melting column and their evolution is of critical importance. We attempt to evaluate these possible ranges by modeling the melting of a mantle source similar to that of the MARK region and fractionation of MORB similar to our estimates of MARK parental magmas. Recent models of MORB formation indicate that melting is near fractional, with low residual melt porosities in the mantle, and that near-fractional melts mix and pool to form MORB (McKenzie, 1984; Klein and Langmuir, 1987; Johnson et al., 1990; Langmuir et al., 1992). These models predict a wide range of melt compositions in the subaxial melting regime. Figure $29 \mathrm{~A}$ illustrates possible trace-element abundances in mantle-generated melts during melting of an N-MORB source. A continuous spinel facies melting model was used with $1 \%$ residual porosity. Thus a near-fractional melting model was employed and is consistent with recent trace-element studies of abyssal peridotites (Johnson and Dick, 1992). The wide range of instantaneous near-fractional melts is illustrated with the field of MARK MORB (J.F. Casey, W. Bryan, and G. Thompson, unpubl. data). Figure 29B shows the compositions of linearly pooled melts for the same melting model, as well as the MARK basalt field. Parental MORB for the MARK area is most adequately modeled as a pooled melt, which has resulted from an average of $12 \%-15 \%$ partial melting. The evolution of such a melt by fractional crystallization would yield the range of MARK MORB trace-element patterns. The evolution of MARK parental MORB is illustrated in the perfect fractional crystallization modeling shown (Fig. 30). Note that the traceelement pattern develops a systematic negative $\mathrm{Sr}$ anomaly because of the fractionation of plagioclase, but for low-pressure fractionation, the abundance of $\mathrm{Sr}$ stays unchanged as other elements become enriched in evolved melts. The parallelism of the trace-element patterns during crystal fractionation and the near constancy of ratios of highly incompatible to less incompatible elements (e.g, $\mathrm{La} / \mathrm{Yb}, \mathrm{Zr} / \mathrm{Y}$ ) during fractionation is a well-known phenomenon of MORB fractionation (e.g., Hanson, 1980; Schilling et al., 1983).

A question of whether ultradepleted to enriched near-fractional melts generated in the subaxial melting column mix before or upon entering subaxial or crustal-level magma chambers remains. To evaluate whether mixing is complete before formation of gabbroic complexes, we compare the compositions of basaltic, gabbroic, and ultramafic rocks. The trace-element composition of cumulates depends on the original melt composition, the partition behavior of each phase in the cumulate, the modal proportions, and the amount and compositions of trapped melt. Of the three important phases, plagioclase and clinopyroxene are the most important receptors of the trace elements plotted in the spidergrams for most samples. plagioclase will tend to have a LREE-enriched pattern with low HREE abundances when crystallized by N-MORB, whereas clinopyroxene will have a LREEdepleted pattern and higher abundances of HREE than plagioclase. The relative abundance of these phase will be important in controlling the trace-element pattern.

Figure 31 includes two plots of the chondrite-normalized $\mathrm{La} / \mathrm{Yb}$ ratio vs. La and vs. Yb depicting both the geochemistry of Leg 153 plutonic rocks and MARK basalts. The range of $\mathrm{La} / \mathrm{Yb}$ ratios in plutonic rocks tends to be much wider than the basaltic rocks as are the absolute abundances of REE. The $\mathrm{La} / \mathrm{Yb}$ ratios and $\mathrm{La}$ and $\mathrm{Yb}$ abundances for clinopyroxene and plagioclase calculated to be in equilibrium with the range of MARK basalts are also shown. The calculations were made using basalt trace-element abundances and the $\mathrm{Kd}$ listed in Table 6. In addition, the $\mathrm{La} / \mathrm{Yb}$ ratios and $\mathrm{La}$ and $\mathrm{Yb}$ abundances expected for adcumulates (without trapped melts), which consist of both $50 / 50$ and 80/20 proportions of plagioclase to clinopyroxene, that are calculated to be in equilibrium with MARK basalts are shown. This $50 / 50$ and $80 / 20$ proportions spans the typical modal range of MARK gabbroic rocks. Although the ratios of the pure endmembers and mixtures of the two phases include the range of chondrite-normalized $\mathrm{La} / \mathrm{Yb}$ ratios represented by the Leg 153 mafic plutonic rocks, the abundances of $\mathrm{La}$ and $\mathrm{Yb}$ tend to be more enriched than would be indicated by mixtures of the pure mineral end-members (i.e., if the $\mathrm{Kd}$ are appropriate). This is interpreted to indicate a 
Figure 29. Modeling of mantle melting. Results of chondrite-normalized trace-element abundances using a continuous melting model with $1 \%$ residual mantle porosity are shown. Near-fractional instantaneous melts are plotted in the upper diagram at $0.5 \%, 5 \%, 10 \%, 15 \%, 20 \%$, and $25 \%$ melting, and linearly pooled melts are plotted at $1 \%, 5 \%, 10 \%, 15 \%, 20 \%$, and $25 \%$ melting. The percent melting refers to Fmax at the top of the melting column in the pooled melt diagram and does not represent the mean extent of melting (see Plank et al., 1995). The field of MARK basalts is stippled. Note the large range in basalt melts generated by fractional melting compared with their pooled hybrids.

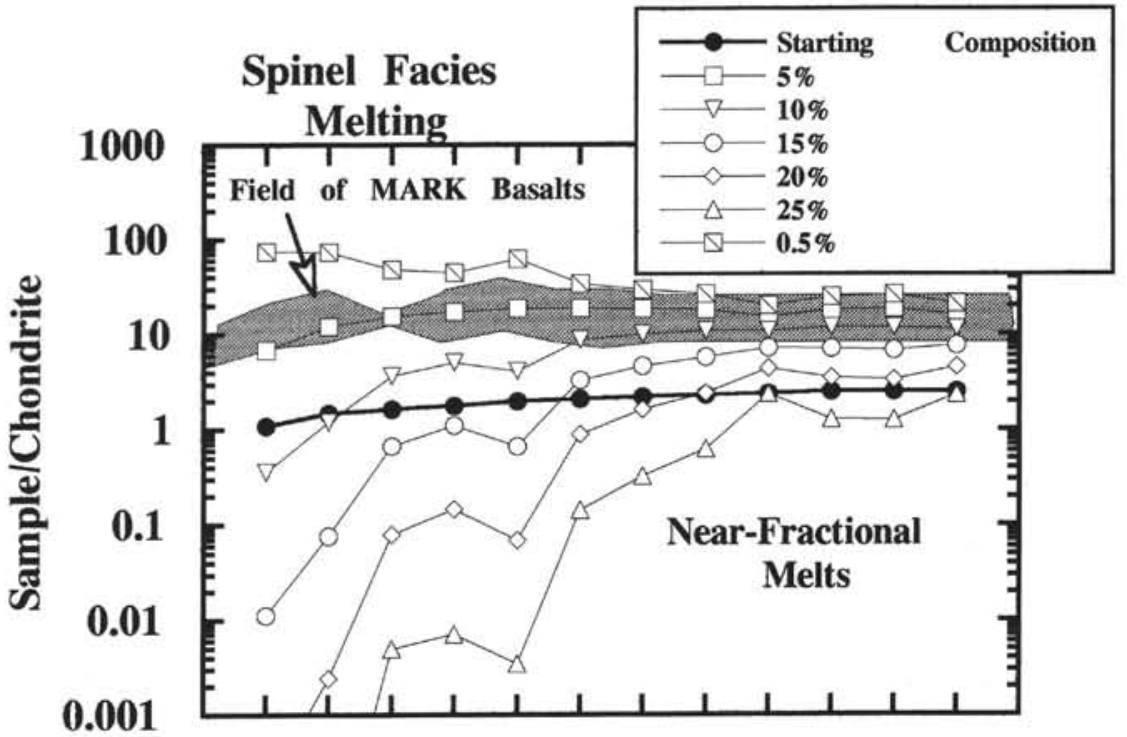

La Ce Sr Nd Zr Sm Eu Gd Ti Dy Er Yb

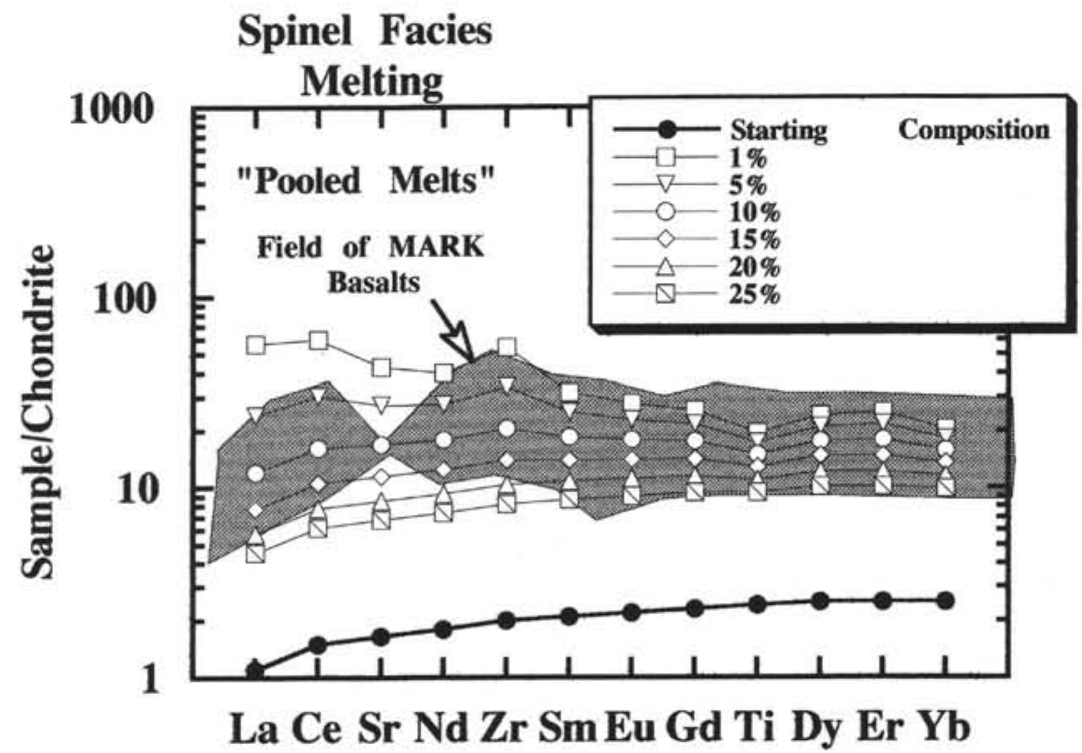

Table 6. Mineral/melt partition coefficients for mantle phases in equilibrium with basaltic magma used in partial melting and fractionation modeling (see Figs. 20, 28, 29, 30, and 31).

\begin{tabular}{llcclll}
\hline Elements & Olivine & Orthopyroxene & Clinopyroxene & Spinel & Garnet & Plagioclase \\
\hline $\mathrm{La}$ & 0.000007 & 0.0005 & 0.0536 & 0.0006 & 0.001 & 0.0415 \\
$\mathrm{Ce}$ & 0.00001 & 0.0009 & 0.0858 & 0.0006 & 0.004 & 0.0297 \\
$\mathrm{Nd}$ & 0.000007 & 0.009 & 0.1873 & 0.0006 & 0.057 & 0.023 \\
$\mathrm{Sm}$ & 0.0007 & 0.02 & 0.291 & 0.006 & 0.5 & 0.0177 \\
$\mathrm{Eu}$ & 0.00095 & 0.03 & 0.35 & 0.35 & 0.0006 & 0.168 \\
$\mathrm{Gd}$ & 0.0012 & 0.04 & 0.4 & 0.0006 & 2 & 0.012 \\
$\mathrm{Dy}$ & 0.004 & 0.06 & 0.442 & 0.0015 & 2.5 & 0.009 \\
$\mathrm{Er}$ & 0.009 & 0.07 & 0.387 & 0.003 & 3 & 0.0079 \\
$\mathrm{Yb}$ & 0.023 & 0.1 & 0.43 & 0.0045 & 4 & 0.007 \\
$\mathrm{Ti}$ & 0.015 & 0.14 & 0.384 & 0.15 & 0.6 & 0.01 \\
$\mathrm{Zr}$ & 0.005 & 0.014 & 0.1234 & 0.07 & 0.3 & 0.0094 \\
$\mathrm{Sr}$ & 0.003 & 0.018 & 0.1283 & 0.0001 & 0.0154 & 1.61 \\
\hline
\end{tabular}

Notes: Olivine data are from Irving (1978) and references cited within, Kelemen et al. (1993) and references cited within, and McKay (1986). Orthopyroxene data are from Dick and Kelemen (1991), Irving (1978) and references cited within, Kelemen et al. (1993) and references cited within, and Weill and McKay (1975). Clinopyroxene data are from Hart and Dunn (1993) and Kelemen et al. (1993). Garnet data are from Irving (1978) and references cited within, Kelemen et al. (1993) and references cited within, and Philpotts and Schnetzler (1970). Spinel data are from Irving (1978) and references cited within, Kelemen et al. (1993) and references cited within, and Stosch (1982). Plagioclase data are from Fujimaki et al. (1984) and Morrison (1990). 
A

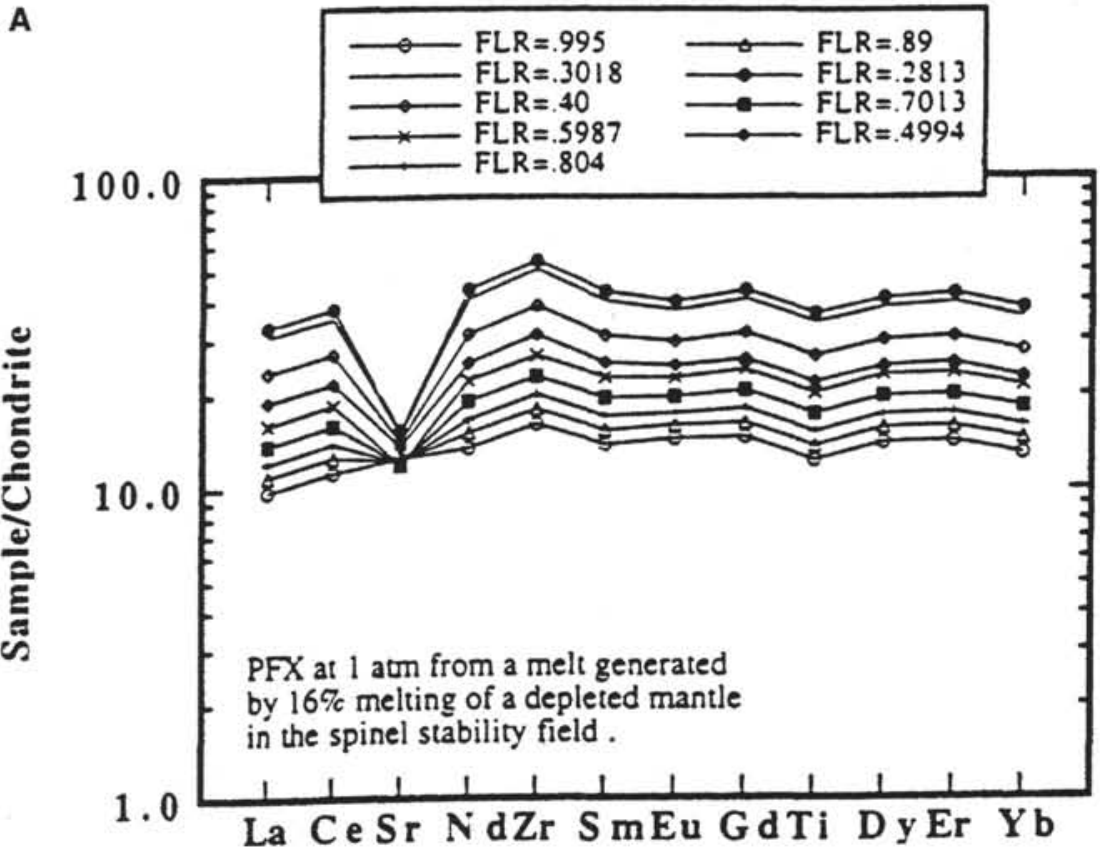

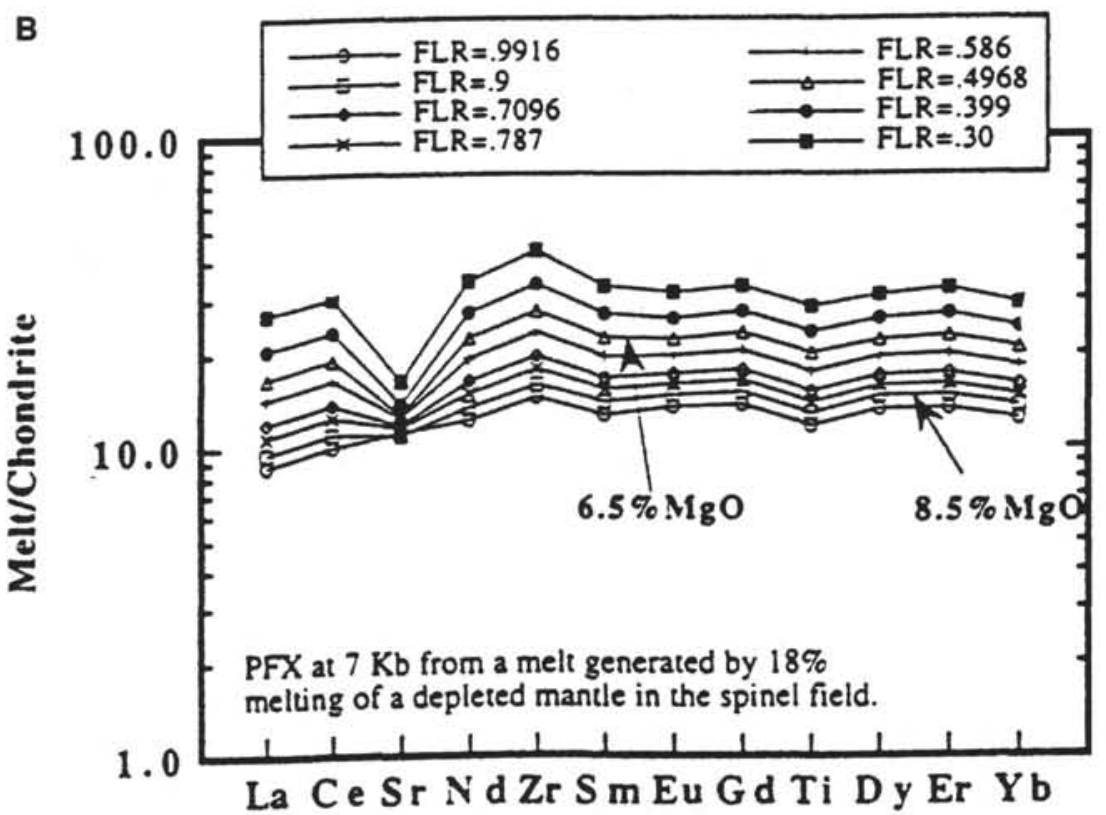

Figure 30. Perfect fractional crystallization (PFX) modeling of the effects of fractionation. Extended REE patterns of closed system fractional crystallization of a parent similar to Leg 153 high-MgO diabase. A. 1 atm. B. 7 kbar. Note that the patterns are parallel throughout their fractionation history, except for Sr. In (A), a negative $\mathrm{Sr}$ anomaly is gradually developed with increased fractionation. Each of the REE patterns subparallel to each other, with $\mathrm{Sr}$ almost invariable. In (B), the patterns look similar to those in $\mathrm{A}$, however, the $\mathrm{Sr}$ content increases with the extent of fractionation because of lesser proportions of plagioclase and higher proportions of clinopyroxene being fractionated. Because $\mathrm{Sr}$ does not vary significantly at low pressures, the bulk $\mathrm{D}$ for $\mathrm{Sr}$ is likely to be $\sim 1$ throughout the fractionation. Higher pressure fractionation will cause more significant variation. Note that the negative anomaly in the basalts would be matched by a positive anomaly in gabbroic cumulates. The extent of fractionation in each diagram is given by the fraction of liquid remaining (FLR). MARK basalts fit within the range of patterns generated by these models. range of trapped melt content within the plutonic rocks and the possibility of a wider array of parental melts than indicated by the MARK basalts.

A subset of the analyses has been compared with modal abundance to examine the modal controls on LREE/HREE (e.g., chondrite-normalized $\mathrm{La} / \mathrm{Yb}$ ) ratios (Fig. 32). In general, the correlations expected by their partitioning behavior exist between plagioclase and clinopyroxene modal abundance and $\mathrm{La} / \mathrm{Yb}$ ratios, but the scatter is significant in $\mathrm{La} / \mathrm{Yb}$ for a given modal percentage. $\mathrm{La} / \mathrm{Yb}$ increases with increasing olivine, which reflects the decreasing modal clinopyroxene and the increased importance of plagioclase in controlling the ratio. The plagioclase/clinopyroxene ratio can be broadly correlated with increasing chondrite-normalized $\mathrm{La} / \mathrm{Yb}$. Although these broad and rather weak correlations exist, the scatter is significant for any single modal abundance and shows that the ratio is influenced, but not solely controlled, by modal abundance.

To examine all of the samples analyzed, elements that vary with increasing modal abundance for particular mineral species can be used as proxies to evaluate modal controls on LREE/HREE (or La/ $\mathrm{Yb}$ ) ratios (Figs. 33A-33D). Plagioclase abundances are related to the bulk $\mathrm{Al}_{2} \mathrm{O}_{3}$ content of the samples. $\mathrm{La} / \mathrm{Yb}$ ratios, as expected, increase with increasing $\mathrm{Al}_{2} \mathrm{O}_{3}$ (plagioclase) content, although there is a wide range of values for a given $\mathrm{Al}_{2} \mathrm{O}_{3}$ content. Sc tends to be concentrated in clinopyroxene and its abundance can be directly related to clinopyroxene modal abundance, as melt $\mathrm{Sc}$ abundances change little with fractionation. A systematic decrease of chondrite-normalized $\mathrm{La} / \mathrm{Yb}$ occurs with increasing modal clinopyroxene (Sc), as expected, although the scatter is wide. The olivine/clinopyroxene ratio 

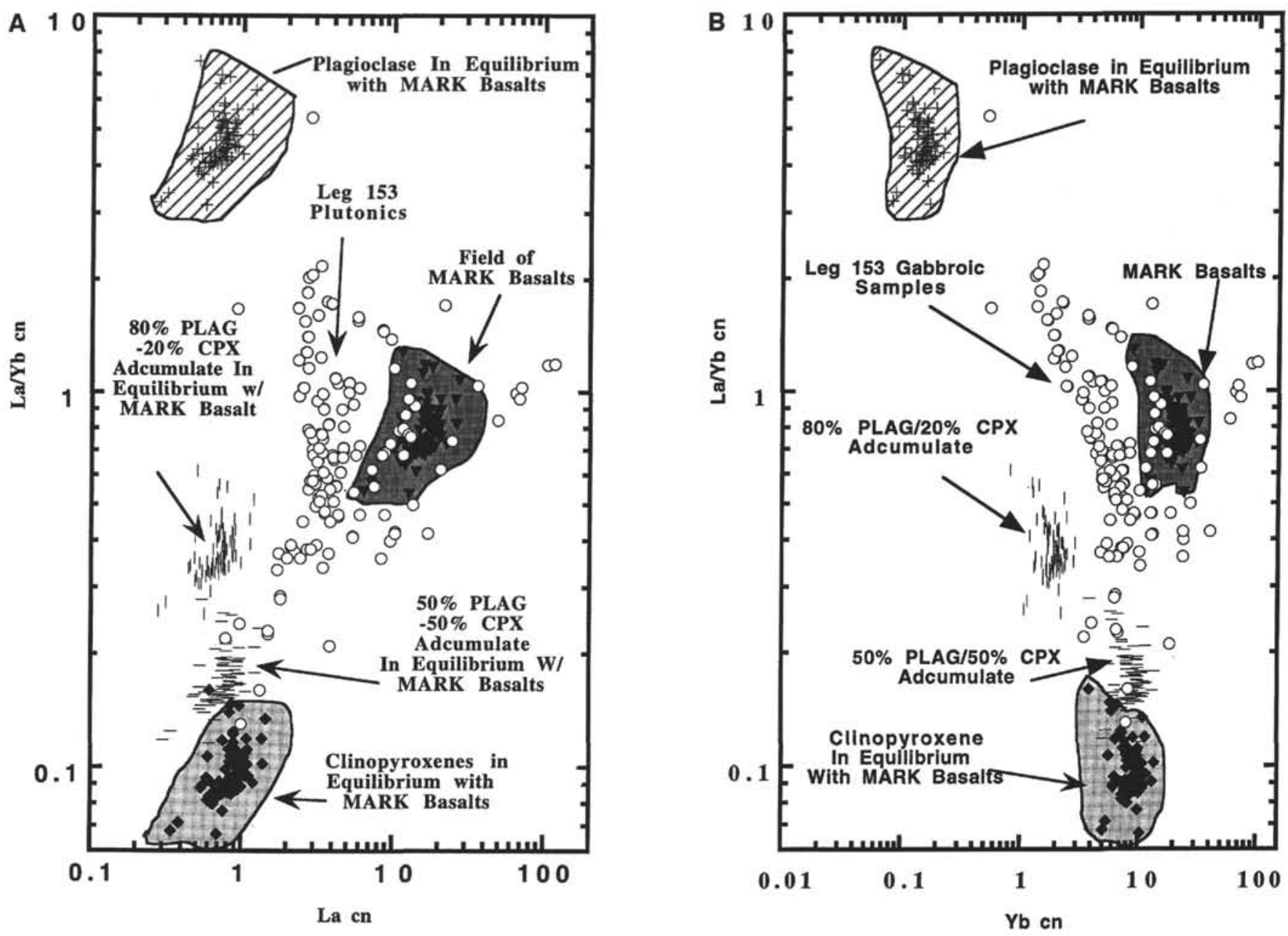

Figure 31. A. Chondrite-normalized La/Yb vs. chondrite-normalized La for MARK mafic and ultramafic plutonic rocks as well as MARK basalts; chondritenormalized $\mathrm{La} / \mathrm{Yb}$ vs. chondrite-normalized $\mathrm{La}$ for calculated plagioclase and clinopyroxene compositions in equilibrium with MARK basalts based on Kd listed in Table 6. B. Chondrite-normalized $\mathrm{La} / \mathrm{Yb}$ vs, chondrite-normalized $\mathrm{Yb}$ for MARK mafic and ultramafic plutonic rocks as well as basalts. Chondrite-normalized $\mathrm{La} / \mathrm{Yb}$ vs. chondrite-normalized $\mathrm{Yb}$ for the same calculated plagioclase and clinopyroxene compositions are also shown. The plutonic rocks have a much wider range in chondrite-normalized $\mathrm{La} / \mathrm{Yb}$ ratios than those of the basalts. Also shown are the expected compositions of an adcumulate with $80 \%$ plagioclase and $20 \%$ clinopyroxene, and an adcumulate with $50 \%$ plagioclase and $50 \%$ clinopyroxene. Note that most samples show higher La and $\mathrm{Yb}$ abundances than predicted by the Kd in Table 6 . The entire variation observed could not be fully explained by subtle changes in the Kd and probably indicate small to large amounts of trapped intercumulus melt.

can be evaluated using the $\mathrm{MgO} / \mathrm{SiO}_{2}$ ratio and $\mathrm{Ni}$ content because both tend to increase with increasing modal olivine. As olivine increases over clinopyroxene as the mafic phase, the importance of plagioclase in controlling chondrite-normalized $\mathrm{La} / \mathrm{Yb}$ ratios increases. There is a weak positive correlation between chondrite-normalized $\mathrm{La} / \mathrm{Yb}$ and olivine $\left(\mathrm{MgO} / \mathrm{SiO}_{2}\right.$ and $\left.\mathrm{Ni}\right)$ content. Thus troctolites commonly show LREE enrichment and chondrite-normalized $\mathrm{La} / \mathrm{Yb}>1$. For all these parameters, however, the range in $\mathrm{La} / \mathrm{Yb}$ and $\mathrm{REE}$ concentration is too large to be simply explained by modal variation.

The affect of accessory phases such as apatite or zircon does not appear to significantly elevate or depress the range of gabbroic chondrite-normalized $\mathrm{La} / \mathrm{Yb}$ ratio outside of that of the MARK basalts. Samples that accumulate zircon and apatite are obvious because of high $\mathrm{Zr}$ and $\mathrm{P}_{2} \mathrm{O}_{5}$ and positive anomalies of each in trace-element patterns. The REE abundances show a positive correlation with bulkrock $\mathrm{P}_{2} \mathrm{O} 5$ and $\mathrm{Zr}$, but no obvious or significant fractionation of the LREE/HREE (Fig. 34).

The addition of pore melt similar in composition to MARK basalt as an intercumulus phase will tend to homogenize the $\mathrm{La} / \mathrm{Yb}$ ratios and draw them closer to the basalt compositions plotted in Figure 30. Because the overall abundances of incompatible elements are much higher in the melts when compared to their crystallization products, small additions can make significant differences in abundances and LREE/HREE ratios. The plot shows that most of the samples that are more enriched in $\mathrm{La}$ and $\mathrm{Yb}$ approach the $\mathrm{La} / \mathrm{Yb}$ ratios of MARK basalts. Presumably, those samples enriched in trapped melt components (orthocumulates) are most similar to MARK basalts in their trace-element characteristics. By far, the widest range of $\mathrm{La} / \mathrm{Yb}, \mathrm{Zr} /$ $\mathrm{Y}$, and $\mathrm{La} / \mathrm{Ce}$ ratios are observed in samples with small amounts of trapped melt. They also tend to be the most incompatible-element depleted (Fig. 35) and the most primitive (highest Mg\#) samples in the suite. These are largely devoid of significant trapped melt components. Thus, inherent differences in modal mineralogy and in primitive melt concentrations may be important in producing the broad array of trace-element abundances and ratios.

In summary, modal mineralogy and the addition of trapped melt to the cumulate samples appear to strongly influence the ratios and abundances of highly incompatible to moderately incompatible elements in gabbroic cumulates, but the differences among melts from which they have originally solidified or which infiltrate them may also be involved in the wide scatter of these ratios. Overall, the narrow range of $\mathrm{Sr}$ abundances in gabbroic rocks that are comparable 

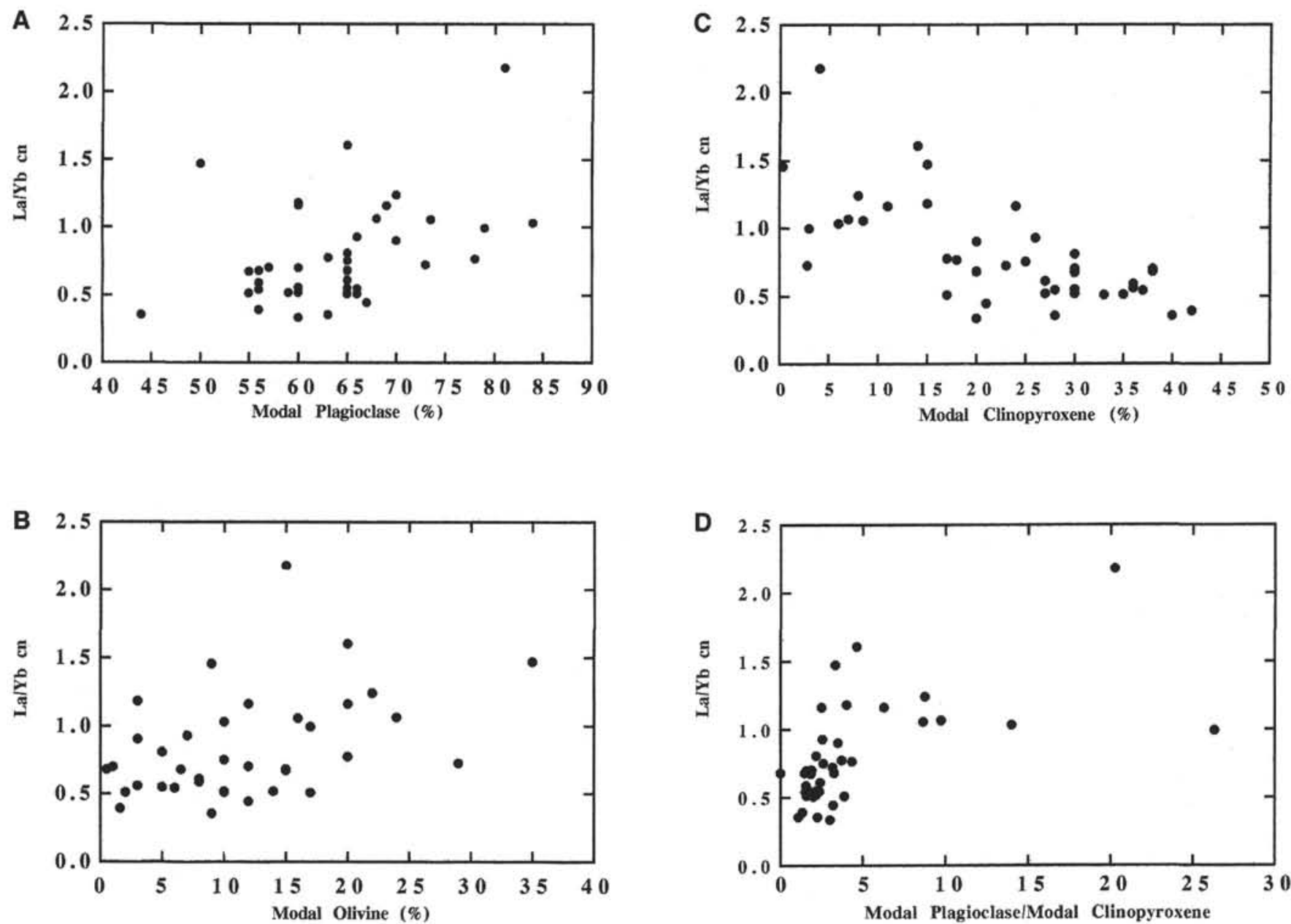

Figure 32. The variation of (A) modal plagioclase, (B) olivine, (C) clinopyroxene, and (D) plagioclase/clinopyroxene with bulk-rock chondrite-normalized La/ $\mathrm{Yb}$ ratios for a subset of the samples analyzed showing part of the variation is likely to be controlled by relative modal abundances.

with basaltic abundances tend to indicate that gabbroic rocks have solidified from a narrower range of parental melts than indicated by plutonic rocks (e.g., pyroxenites) within Holes 920B and 920D from the ultramafic massif. Because the bulk D is likely close to 1 for $\mathrm{Sr}$ during fractionation, the similarity of the range in gabbroic samples from Sites 921, 922, and 923 with the range of basaltic samples inicates they have broadly similar parents. $\mathrm{Sr}$ is not expected to significantly increase or decrease in abundance during low-pressure fractionation (Fig. 30). In gabbroic rocks the abundance of Sr generally falls within the range from 10 to $11 \times$ chondrite (Figs. 22, 23, 25, 27), which is approximately the same range as that observed in MARK basalts (Fig. 15). Below we outline further evidence for the overall range of parental melts generated and those formed by mixing of mantle melts based on the mineral chemistry of clinopyroxene in residual mantle and gabbroic samples, and their possible involvement in producing trace-element ratio variations.

\section{$\mathrm{Na}_{2} \mathrm{O}$ ABUNDANCES IN MELTS CALCULATED TO BE IN EQUILIBRIUM WITH CLINOPYROXENE FROM LEG 153 RESIDUAL PERIDOTITES AND MAFIC ROCKS}

To estimate whether melts in equilibrium with Leg 153 ultramafic and mafic plutonic rocks were similar to MARK MORB erupted at the surface, we compare the range of MARK glass composition (Bryan et al., 1994) with the range of melts calculated from mineral chem- istry data of Leg 153 clinopyroxene in both residual mantle and plutonic rocks. These data are derived from the same samples for which bulk-rock data were collected. Calculations are based on the clinopyroxene partition coefficients established for $\mathrm{TiO}_{2}, \mathrm{Na}_{2} \mathrm{O}$ and $\mathrm{FeO} /$ $\mathrm{MgO}$ in MORB experimental data (Roeder and Emslie, 1970; Kinzler and Grove, 1992). In Figure 36, the compositions of MARK basalts are represented on $\mathrm{FeO} / \mathrm{MgO}$ vs. $\mathrm{Na}_{2} \mathrm{O}$ Harker diagrams. In addition, calculated melts in equilibrium with clinopyroxene from ultramafic and mafic rocks in Holes 920B and 920D in the ultramafic massif and from Sites 921,922 , and 923 are plotted separately to facilitate direct comparison with basalts. Perfect fractional crystallization trends for Site 920 diabases and the most primitive MARK glass (Bryan et al., 1994) are also shown. The small increases in $\mathrm{Na}_{2} \mathrm{O}$ with increasing fractionation of the basalts (MORB) allows comparison of the incompatible-element abundances of melts in equilibrium with plutonic and residual harzburgite clinopyroxene with those in melts parental to MORB in the MARK area.

\section{Residual Abyssal Peridotites-Site 920}

Residual mantle clinopyroxene is typically too depleted to have been in equilibrium with a MARK basalt parent. As a consequence of near-fractional melting, melts in equilibrium with these harzburgites would be expected to be ultradepleted with respect to MARK glasses. Because abyssal peridotites in Holes 920B and 920D generally represent the residuum of partial melting and are sampled from the top of the residual melting column, they are likely to be far more depleted than indicated by the compositions of average pooled melts (MARK 

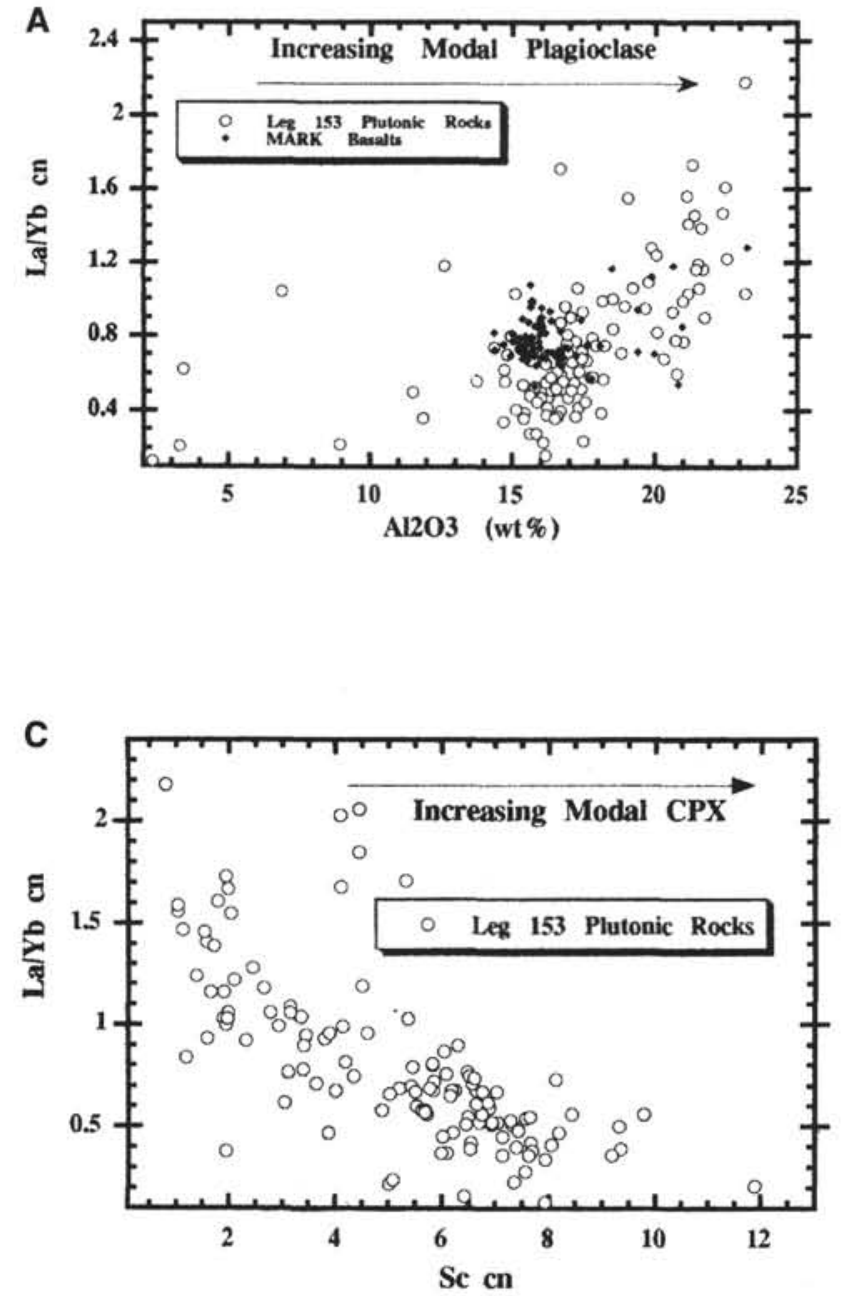
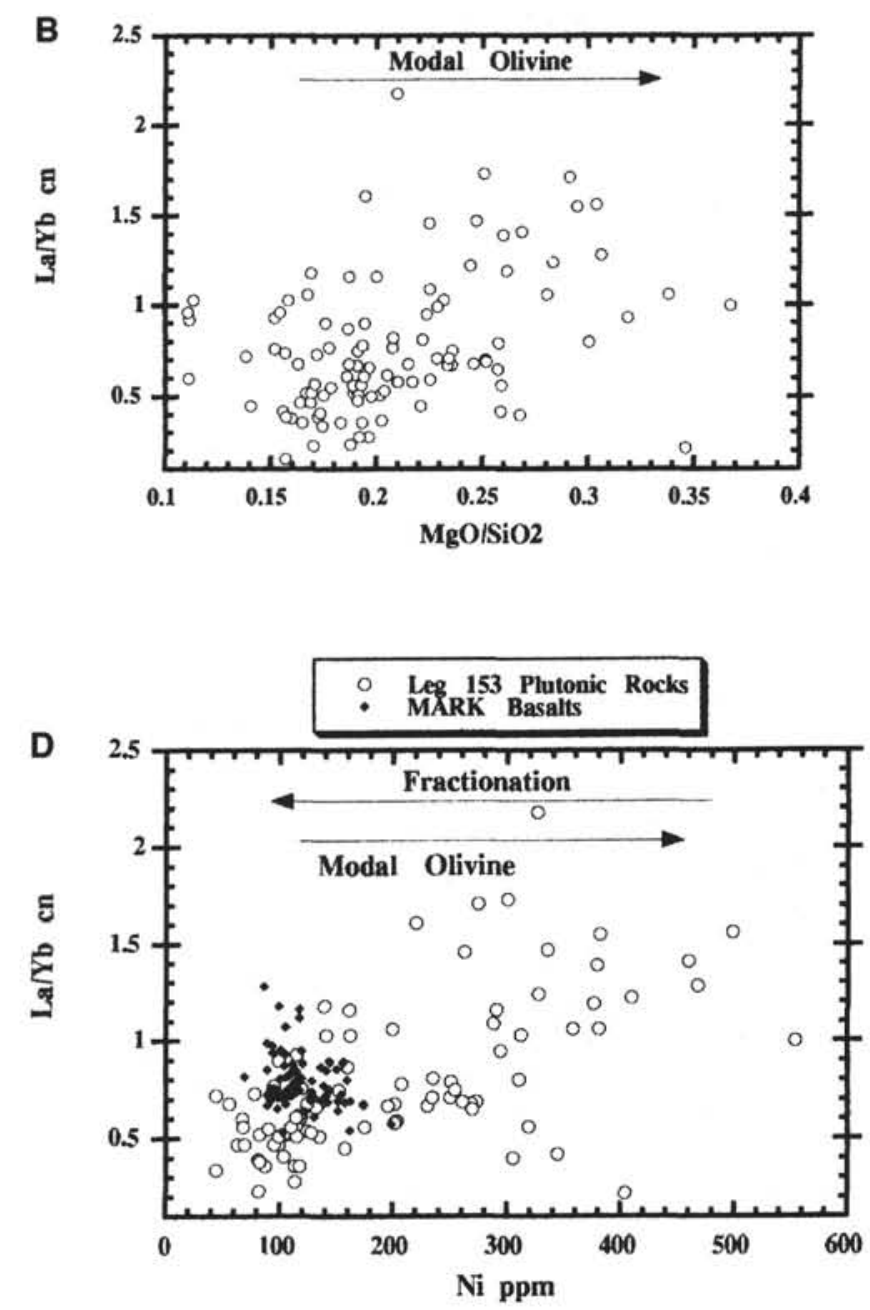

Figure 33. Chondrite-normalized (cn) $\mathrm{La} / \mathrm{Yb}$ ratios vs. $\mathrm{Al}_{2} \mathrm{O}_{3}$ wt\% (A); $\mathrm{MgO} / \mathrm{SiO}_{2}$ (B), chondrite-normalized Sc (proxy for clinopyroxene) (C), and Ni content for Leg 153 plutonic rocks (D). $\mathrm{Al}_{2} \mathrm{O}_{3}$ is used as a proxy for plagioclase content, $\mathrm{Sc}$ for pyroxene content, and $\mathrm{MgO} / \mathrm{SiO}_{2}$ and $\mathrm{Ni}$ for olivine content. Also shown are the compositions of MARK basalts. Modal controls are obvious, although the significant scatter suggest other factors are involved in controlling bulk-rock chondrite-normalized $\mathrm{La} / \mathrm{Yb}$.

glasses). This is consistent with the mineral chemistry and bulk-rock data. These data are also consistent with ion probe data for clinopyroxene from abyssal peridotites as described by Johnson et al. (1990) and Johnson and Dick (1992). Calculated ultradepleted melt could not evolve into MARK basaltic compositions by fractional crystallization because the slope of $\mathrm{Na}_{2} \mathrm{O}$ increase is much too shallow.

\section{Ultramafic Plutonic and Gabbroic Rocks-Site 920}

Clinopyroxene in plutonic rocks including primitive wehrlites, websterites, and clinopyroxenites from Leg 153 Site 920 are in equilibrium with a range of liquids that include those that are both ultradepleted to significantly enriched in $\mathrm{Na}$ and $\mathrm{Ti}$ with respect to MORB melts. Like the trace-element patterns for plutonic rocks from Sites 921,922 , and 923 , the abundance of $\mathrm{Na}_{2} \mathrm{O}$ shows the highest diversity in primitive samples. There is little overall change in $\mathrm{FeO} / \mathrm{MgO}$ of the melts, which tend to be primitive and close to equilibrium with mantle olivine. This trend in Site 920 melts is highly oblique to the melt fractionation trend predicted on the basis of perfect fractional crystallization modeling of MARK basaltic glass. In general, the mantle intrusive rocks provide evidence of a range of parental melts transitional between the melts in equilibrium with ultradepleted residual rocks and MARK basalts and gabbroic rocks from Sites 921 ,
922, and 923. In some cases Site 920 intrusive rocks tend to be somewhat more enriched in $\mathrm{Na}$ and $\mathrm{Ti}$ than MARK basalts.

\section{Gabbroic Rocks-Site 921, 922, and 923}

The progressive enrichment in $\mathrm{Na}_{2} \mathrm{O}$ content without significant change in $\mathrm{FeO} / \mathrm{MgO}$ in melts calculated to be in equilibrium with clinopyroxene in ultramafic and gabbroic rocks at Site 920 , gives way to an overall trend of slightly increasing $\mathrm{Na}_{2} \mathrm{O}$ with marked increases $\mathrm{FeO} / \mathrm{MgO}$ content for the calculated liquids. This trend is nearly parallel and overlaps with the MARK glasses and the perfect fractional crystallization trends plotted. The swath of calculated melts is wider by far than the trend displayed by the MARK glasses and several of the calculated melts are either too enriched or too depleted in $\mathrm{Na}_{2} \mathrm{O}$ to be close to equilibrium with MARK glasses. However, the average trend of melts in equilibrium with gabbroic clinopyroxene from Sites 921,922 , and 923 is nearly identical to the perfect fractional crystallization trend defined by the primitive MARK glass and Hole 920B diabase. These data are consistent with the model that MORB represents an aggregate of near-fractional melts pooled and homogenized at crustal levels to form MORB. It is likely that the bulk-rock data and the mineral chemistry data can best be explained as the crystallization or equilibrium products of a broad array of near-fractional or poorly 

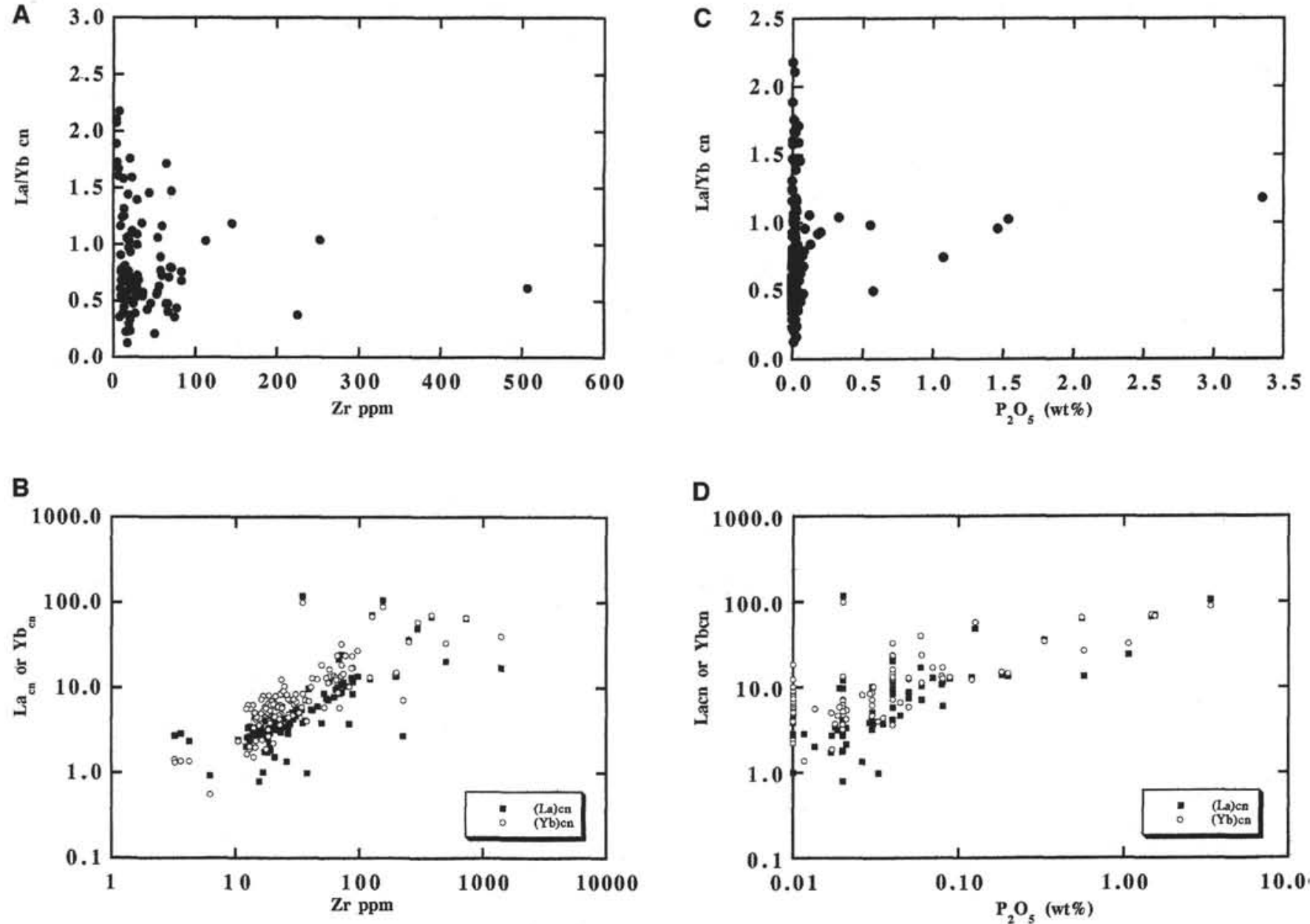

Figure 34. Chondrite-normalized (cn) La/Yb vs. $\mathrm{Zr}$ (A), chondrite-normalized $\mathrm{La}$ and chondrite-normalized $\mathrm{Yb}$ vs. $\mathrm{Zr}(\mathbf{B})$, chondrite-normalized $\mathrm{La} / \mathrm{Yb}$ vs. $\mathrm{P}_{2} \mathrm{O}_{5}$ (C), and chondrite-normalized $\mathrm{La}$ and chondrite-normalized $\mathrm{Yb}$ vs. $\mathrm{P}_{2} \mathrm{O}_{5}$ (D) illustrating samples likely to have accumulated accessory phases zircon and apatite and the lack of marked fractionation of the LREE/HREE caused by this accumulation. $\mathrm{Zr}$ and $\mathrm{P}_{2} \mathrm{O}_{5}$ are strongly correlated with LREE and HREE abundances.

mixed melts derived from the melting column (Holes 920B and 920D) and a narrower array of more completely mixed melts (Sites 921, 922, and 923) similar to MORB that has erupted in the MARK region. A comparison of the $\mathrm{Na}_{2} \mathrm{O}$ abundances of MARK glasses and melts calculated to be in equilibrium with clinopyroxene from the ultramafic massif and gabbroic massif is shown in Figure 37. It depicts the narrowness of the range in MARK MORB in comparison calculated melts.

\section{Implications}

Recent melting models predict this broad array of melts, but it is impossible to document them in the hybrid melt (i.e., MORB). Direct evidence of their existence comes from recent studies of melt inclusions in primitive MORB (e.g., Sobolev and Shimizu, 1992; Sobolev et al., 1992) and plutonic assemblages represent yet another method of identifying this broad array of melts. It appears that studies concentrated on primitive mantle intrusive rocks may yield the broadest arrays of such melts. Figure 35 demonstrates that there is also a complete continuum of clinopyroxene compositions between residual mantle and evolved gabbros, and no compositional gap. This continuum is similar to that sampled in ophiolite complexes within the transition zone between gabbros and residual mantle assemblages (Elthon and Casey, 1985; J.F Casey, unpubl. data). Elthon and Casey (1985) first pointed out the probable existence of primary melts ultradepleted in highly incompatible elements from evidence from cli- nopyroxene within the transition zone of the Bay of Islands Ophiolite. With respect to MORB-like trace-element abundance in basalts in the Bay of Islands Ophiolite Complex (Casey et al., 1985), these samples showed evidence of the existence of very ultradepleted melts. These melts are similar to those documented within abyssal peridotites (Johnson and Dick, 1990), MORB glass melt inclusions (Sobolev and Shimizu, 1993), and other oceanic ultramafic cumulates (Ross and Elthon, 1993). In the MARK region these samples are represented by mantle intrusive rocks and residual harzburgites in Holes 920B and 920D. They are in equilibrium with a range of melts from enriched to ultradepleted.

Although the number of sites along the Mid-Atlantic Ridge from which microprobe data from clinopyroxene in mantle peridotites and gabbroic rocks and analyses of basaltic glass are available is small, Figure 38 depicts a compilation of available data (J.F. Casey and S. Silantyev, unpubl. data), showing the range of $\mathrm{Na}_{2} \mathrm{O}$ calculated to be in equilibrium with clinopyroxene of oceanic gabbros and ultramafic rocks (residual and intrusive) in comparison with basaltic glass compositions. Although the database is larger in the MARK region, the data from other regions show very similar trends. Ultramafic rocks yield calculated melts that tend to be ultradepleted with respect basaltic glasses, but show wide variation from ultradepleted to enriched. Gabbroic samples yield calculated melts that tend to be more concentrated and more similar to basaltic glasses, but span a wider range from more depleted to more enriched than basaltic glasses. Part of the range in gabbroic samples is due to the fact that they tend to cover a 

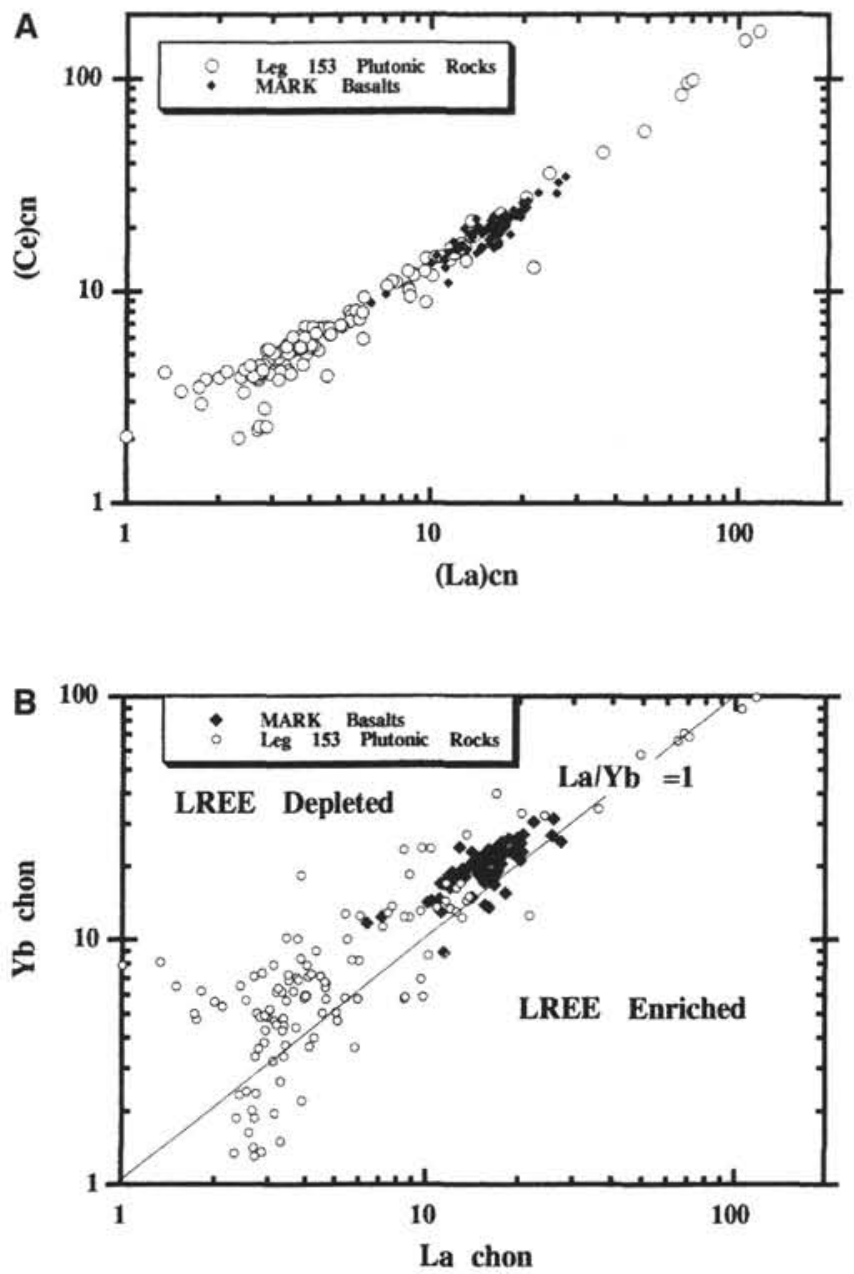

Figure 35. A. Chondrite-normalized Ce vs. chondrite-normalized La. B. Chondrite-normalized $\mathrm{Yb}$ vs. chondrite-normalized $\mathrm{La}$. The plutonic rocks have a much wider range of these elements or elemental ratios and abundances than the MARK basalts. The least fractionated samples show the broadest range of values.

wider range of fractionation than glass populations, but some of the range appears to be due to incomplete pooling of melts in the plutonic environment. These data support the notion that a wide array of melts are generated in the polybaric melting columns, that the melting column is an open system to a variety of melts generated deeper in the mantle, and that these melts pool at plutonic levels in the crust or upper mantle.

\section{TECTONIC IMPLICATIONS OF LOW EXTENTS OF MELTING AND A SHALLOW SPINEL FACIES MELTING REGIME}

In comparison to adjacent regions of the Mid-Atlantic Ridge, the extent of melting and mantle depletion appear low. Melting appears to take place within the spinel facies, indicating a low mantle temperature and low volatile contents in the mantle. Fmax is calculated to range from $15 \%$ to $20 \%$ in the peridotites, and up to $14 \%$ in the basalts. The average extent of melting in the melting column is half of Fmax. Thus, the bulk of the evidence presented here indicates that geochemical variations in the MARK region tend to indicate that the length of the melting column is short, the depth of initial melting is shallow, and the temperature of the subaxial mantle is low. Region- ally, the MARK area would appear to represent one of the minima in melt supply and probable "magmatic" crustal thickness along the Mid-Atlantic Ridge (also see Klein and Langmuir, 1987). Geochemical studies based on results of ODP Holes 395 and 396 (Bougault et al., 1979) suggest that these mantle source and melting characteristics have persisted at this latitude for at least the last $10 \mathrm{~m} . \mathrm{y}$. This longterm minimal melt supply has probably resulted in a discontinuous or cyclic variation superimposed on the low average melt supply (Karson et al., 1987, 1990). Because extension at mid-ocean ridges can be accommodated only by magmatism or tectonic stretching of the lithosphere, the rather low melt supply in the region has significance to both the tectonic regime and crustal formation and architecture (Karson et al., 1987; Casey et al., 1992a; Cannat, 1993; Cannat et al., 1995; Cannat and Casey, 1995), as well as crustal deformation and disruption in the MARK region. Periods of magma drought or minimal magma supply will be characterized by extensive lithospheric stretching and probably result in incomplete formation of a continuous magmatic layer (Cannat, 1993; 1995). Karson and Dick (1983) and Karson et al. $(1987,1990)$ have attributed exposures of ultramafic and gabbroic massifs along the western wall of the Mid-Atlantic Ridge in the MARK area to be the result of extensive tectonic stretching and development of an asymmetric low-angle detachment zone along which deep levels of a fully formed crust and upper mantle are disrupted, unroofed, and exposed. Other mechanisms proposed for these exposures are more complex, and involve tectonic extension coupled with incomplete formation of the magmatic crustal layer. These models involve, in part, the formation of gabbroic bodies in the subaxial mantle. Gabbroic bodies are enclosed within a mantle matrix and are brought toward the surface during mantle upwelling and tectonic stretching, often being disrupted during the tectonic stretching phase (Cannat, 1993; Casey et al., 1992b; Cannat and Casey, 1995). Figure 39 depicts the possible magmatic, crustal architecture and tectonic consequences of reduced melt supply. Melt supply should have consequences for not only how and whether the lower plutonic crustal layer forms at all, but whether rocks typical of the crust mantle transition zone in ophiolites form at the base of the plutonic section or are distributed within the mantle section. These geometries also have significance for the melt delivery processes and the efficiency of the subaxial plumbing system to effectively pool and homogenize mantle melts. Finally, it has significance on where melts in the subaxial environment start to solidify and form cumulates, at crustal or upper mantle levels. This is significant in understanding why observables like crustal thickness estimates based on seismic results do not necessarily match crustal thickness estimates of partial melting based on basalt geochemistry.

\section{DISCUSSION}

The genesis of MORB basalts is now widely regarded to result from the simultaneous extraction of small melt increments within the subaxial melting column over a broad pressure range. The melt fraction retained in the residuum is believed to be small (McKenzie, 1984; Johnson and Dick, 1992), and the process can be regarded as near fractional, leading to the formation of a wide array of parental melts that presumably mix within the subaxial plumbing system to produce a rather homogeneous MORB at the surface. The wide range of bulk and mineral chemistries observed in Leg 153 gabbros and peridotites reflects this wide array of melts. The sampled array of calculated melts and the wide array of trace-element ratios suggest that this mixing is incomplete within the mantle section and that both enriched and ultradepleted melt compositions fractionate either in the mantle or in the mafic crust. If these melts fractionate before mixing with the complete range of melts produced in the melting column, they can be reflected in both bulk-rock and mineral chemistry results. Trace-element studies by ion microprobe and laser ablation induc- 


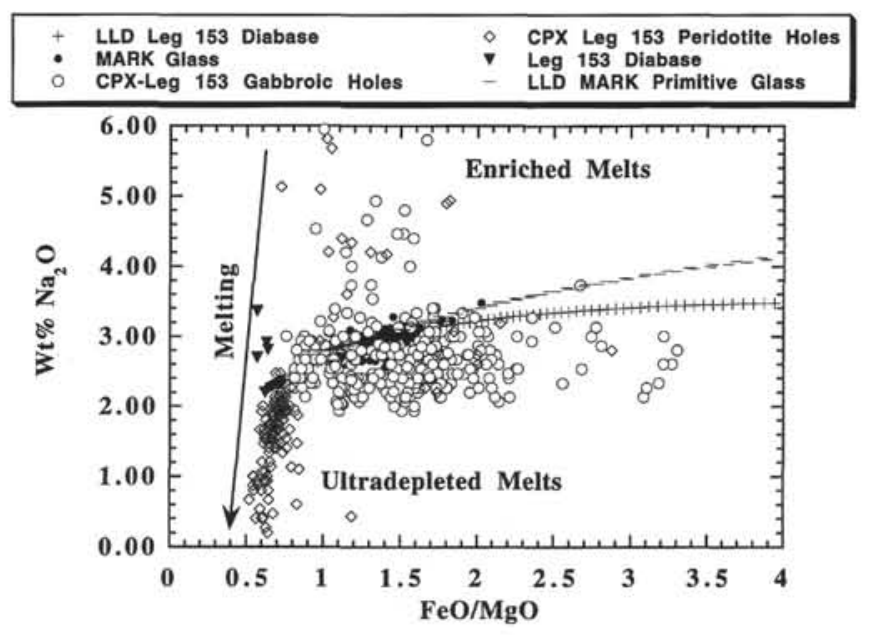

Figure 36. $\mathrm{Na}_{2} \mathrm{O}$ wt $\%$ vs. $\mathrm{FeO} / \mathrm{MgO}$ for calculated liquid compositions in equilibrium with gabbroic clinopyroxene from Sites 921, 922, and 923 and with clinopyroxene in ultramafic rocks from Site 920, including residual harzburgites and pyroxenites. The range of MARK glasses is also shown. The liquids in equilibrium with clinopyroxene in both gabbroic rocks and ultramafic rocks span a wide range of compositions from ultradepleted to very enriched. Liquids in equilibrium with clinopyroxene of gabbroic rocks dominantly cluster close to MARK glasses and calculated liquid lines of decent for a primitive MARK glass and a Hole 920B diabase.

tively coupled plasma-mass spectrometry (ICP-MS) should prove important in understanding the complete range of petrogenetic processes and melt compositions.

The compositions of the MARK region gabbroic and ultramafic samples appear to span the compositional range of plutonic rocks generally recovered from the oceanic ridges, but in a single location. Samples recovered also appear to span the lithologic and compositional range of samples from ophiolites with MORB-like compositions. The spiked trace-element patterns of the plutonic rocks characteristic of the foundations of the oceanic crust may be related to the spiked patterns typically observed in subduction zone lavas to some extent. These spiked patterns are most often attributed to slab components, which are typically modeled as the volatile, basaltic, and sediment components of the downgoing slab. Plutonic rocks show very different patterns from basalts. They can be an important component of the slab, as plutonic rocks on average may make up over two-thirds of the oceanic crustal stratigraphy. $\mathrm{Sr}, \mathrm{Eu}, \mathrm{Zr}$, and $\mathrm{Ti}$ anomalies are among the most prominent anomalies identified in the plutonic assemblages and all are known from certain island arc environments. Compositions can also have certain consequences for isotopic systems. For example, the low concentrations of $\mathrm{Rb}$ and high concentrations of $\mathrm{Sr}$ in cumulates results in an important fractionation in $\mathrm{Rb} / \mathrm{Sr}$ ratios from those in basalts. The average $\mathrm{Rb} / \mathrm{Sr}$ ratio is $\sim 0.008$ for gabbroic rocks, whereas it is significantly higher $(\sim 0.023)$ for MARK basalts. Recycling of the ocean crust and long-term mantle evolution of a lower crustal component may produce ${ }^{87} \mathrm{Sr} /{ }^{86} \mathrm{Sr}$ ratios significantly more depleted than its upper crustal basaltic counterpart, and may in part help to explain HIMU (Sun and McDonough, 1989) depletions in these ratios.

Unlike ophiolites that have well-defined and ordered stratigraphies in their lower sections that include upward sequences of residual mantle, followed by continuous to semicontinuous sections of ultramafic cumulates, mafic-ultramafic transition zones, and gabbroic plutonic rocks that lie below a hypabyssal-extrusive carapace, the slow-spreading lithosphere with a diminished melt supply formed in the MARK area may have the ultramafic cumulates and transition zone primitive cumulates masked within the residual mantle as broad

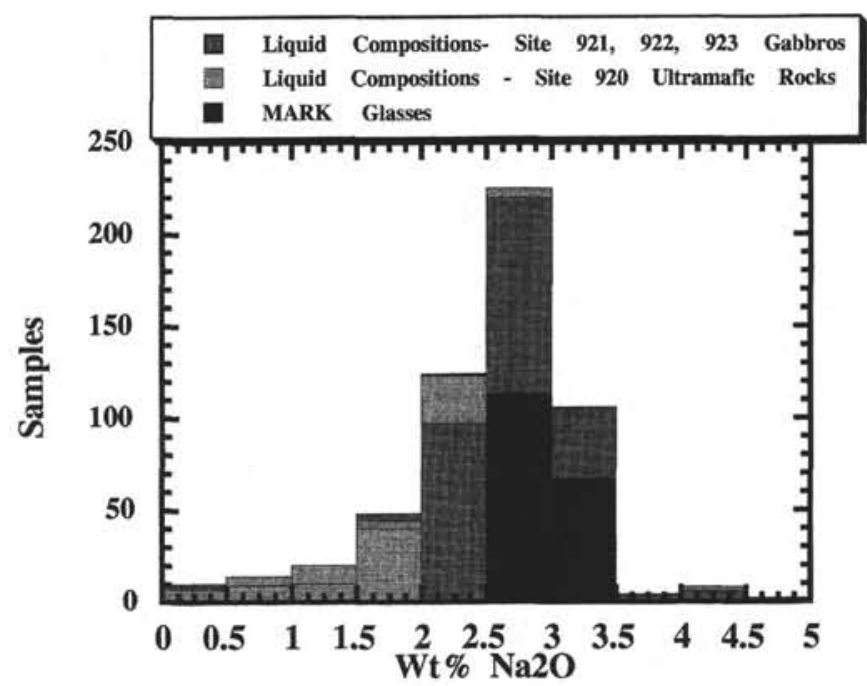

Figure 37. Histograms showing the range of $\mathrm{Na}_{2} \mathrm{O}$ wt $\%$ of liquid compositions in equilibrium with clinopyroxene in gabbroic and ultramafic rocks. Also shown are the very narrow ranges of basalt compositions in the MARK region.

zones or dikelets where olivine, clinopyroxene, and plagioclase have crystallized. A characteristic feature of these intrusive rocks will be that the Mg\# of clinopyroxene (92-84) and forsterite values of olivine (90-83) will be lower than that in the residual mantle. Trace-element signatures are also variable in comparison with the average residual mantle, ranging from highly enriched to highly depleted in incompatible elements and certain elemental ratios such as $\mathrm{La} / \mathrm{Yb}$ and $\mathrm{Zr} / \mathrm{Y}$. Some of these melt products show evidence that they were highly fractionated before intrusion and may have reacted with the residual mantle. This type of mantle refertilization may likewise have long-term effects on the bulk isotopic evolution of the mantle.

Inferences from the data presented here include the likelihood of a spectrum of crustal and upper mantle architectures at mid-ocean ridges that result from variations from continuous to diminished and finally to discontinuous melt supplies. The classical ophiolite stratigraphy may be produced at high spreading rates or under conditions where magma supply is plentiful and mechanical stretching is unimportant. When magma supply is extremely low, as apparent in the MARK area, no organized crustal stratigraphy may evolve and discrete mafic magma chambers may be surrounded by mantle wallrock and exposed at the surface as a consequence of mechanical stretching. The most extreme cases of poorly developed crustal architecture appear to occur where widespread mantle exposures exist along the rift valley, where melt supply is very low, and where mechanical extension dominates. The $15^{\circ} 20^{\prime}$ Transform (Casey et al., 1992b; Cannat, 1993; Cannat and Casey, 1995) and the Romanche Transform (Bonatti et al., 1992, 1993) are such regions where widespread ultramafic outcrops exist. Evidence of near-fractional melts is likely to be found within the veins and plutons that intrude mantle sections. The range of possible crustal architectures and consequences of varying melt supplies are illustrated in the Figure 39.

\section{CONCLUSIONS}

1. The MARK region is characterized by the eruption of high$\mathrm{Na}_{8.0}$ and low- $\mathrm{Fe}_{8.0}$ basalts. These indicate low degrees of average melting $(F \max =\sim 12 \%-15 \%)$ and a shallow melting regime with initial melting at $\sim 45-60 \mathrm{~km}$ depth.

2. Trace-element patterns for MARK basalts and Leg 153 primitive diabase indicate that $\sim 12 \%-15 \%$ of near-fractional partial 


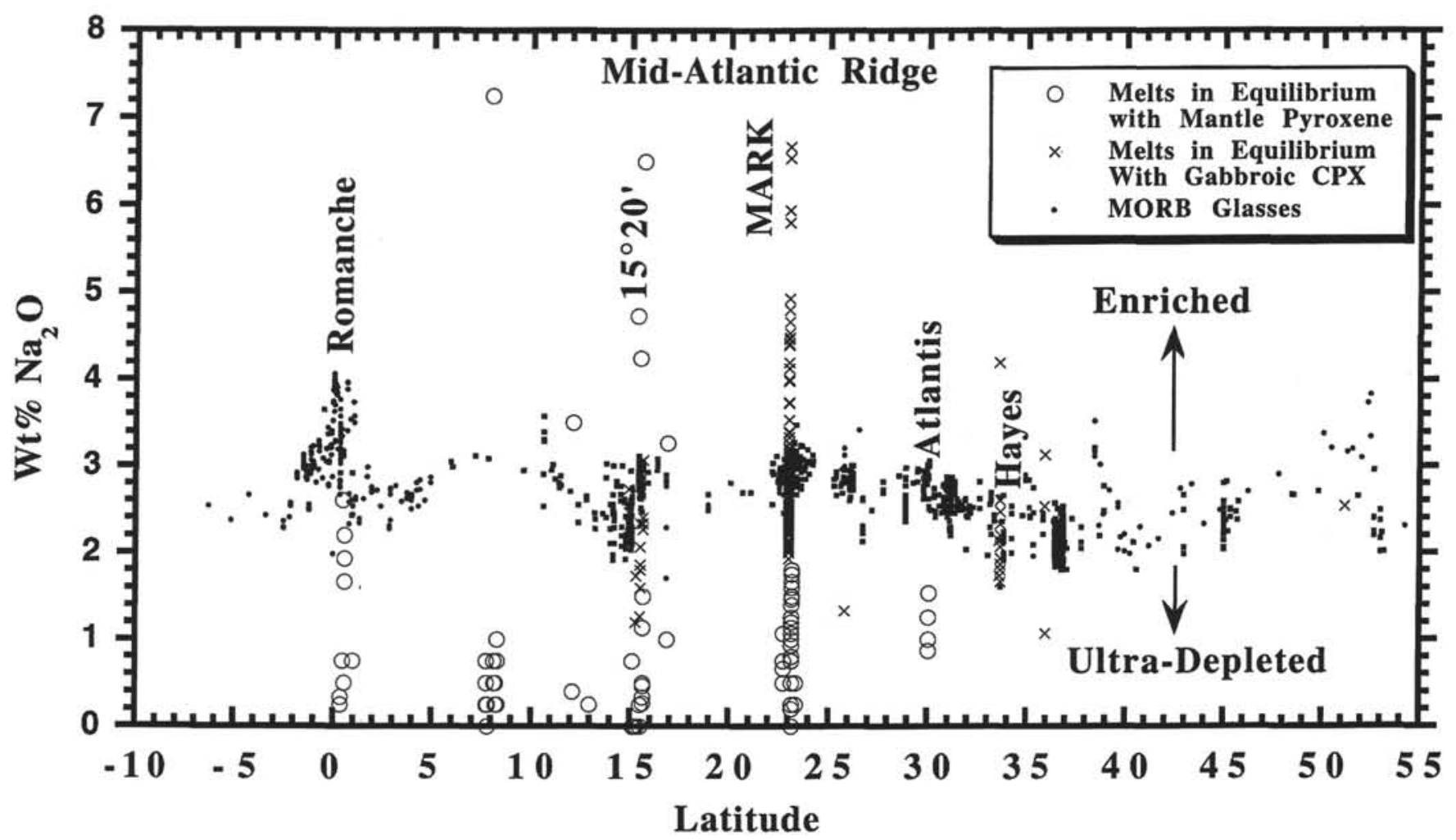

Figure 38. Melts calculated to be in equilibrium with clinopyroxene from the Mid-Atlantic Ridge from abyssal peridotites and gabbroic rocks compared with the $\mathrm{Na}_{2} \mathrm{O}$ content of basaltic glass. Peridotite- and gabbro-derived compositions show a complete range from ultradepleted to enriched relative to MORB, not only in the MARK region, but elsewhere along the Mid-Atlantic Ridge. Data sources include J.F. Casey, S. Silantyev, and W. Bryan (unpubl. data) and references cited in Figures 3 and 5.

Figure 39. Possible models for crust formed where melt supply is robust (fast-spreading centers) to starved (slow-spreading centers). The spectrum of models from left to right illustrates changes in crustal thickness that might occur from high to low melt supplies. In addition note the crystallization of melts within the mantle section where melt supplies are low and the thickness of the mechanical lithosphere is large.
Evolution of Near-Fractional Melts
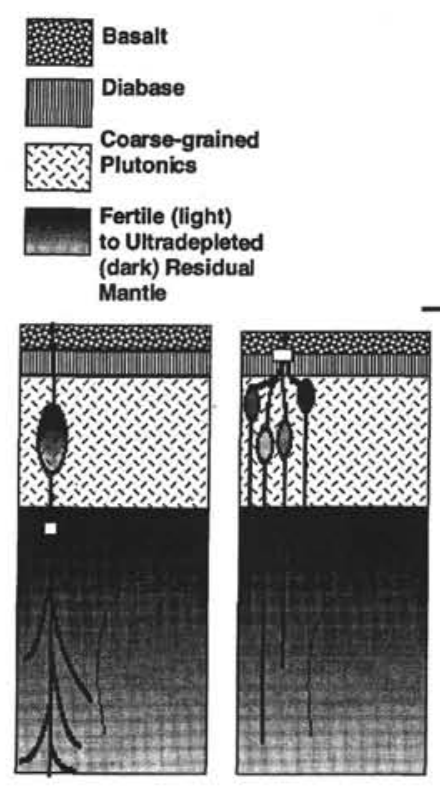

Chambers With Magma Mixing and Fractional Crystallization

Chambers With Fractional Crystallization of Various Near-Fractional or Incompletely Pooled Melts

Aggregation and Pooling of Melts In Magma Conduts During Melt Transport Decreasing MagmaSupply
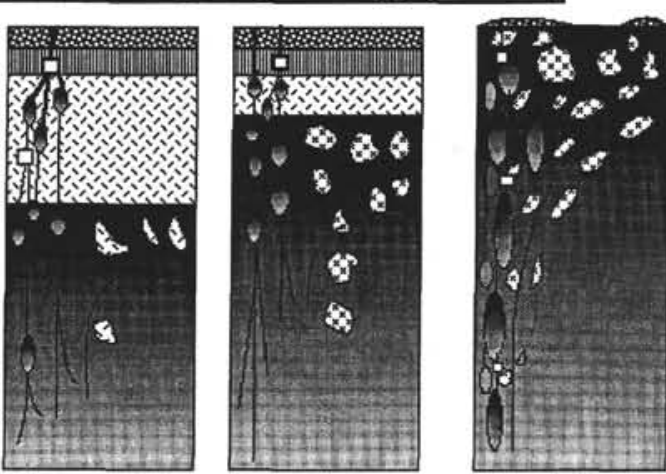

melting affected the shallow mantle and was restricted to the spinel facies (i.e., with little or no garnet facies melts involved).

3. Basalt geochemistry data indicate that the MARK region is characterized by low melt supply and a thin crustal component relative to adjacent portions of the Mid-Atlantic Ridge. The crust may be easily disrupted leading to exposures of both mantle and gabbroic massifs because of tectonic stretching of the mechanical lithosphere because of this low melt supply.

4. Bulk-rock and mineral chemistry studies suggest that residual mantle had been significantly depleted in major-element constituents (e.g., $\mathrm{Al}_{2} \mathrm{O}_{3}$ ) with respect to a fertile MORB mantle source with melting up to an Fmax of $\sim 15 \%-20 \%$. This ex- 
ceeds the average melting determined by using pooled melt compositions (MORB glasses), because the residual mantle rocks sampled represent the ultradepleted top of the residual melting column. In addition, clinopyroxene may have been locally depleted in the residuum because the melting column is an open system to melts generated deeper in the melting column.

5. The residual mantle can best be modeled as an open system (Johnson and Dick, 1992) that is infiltrated by a range of melt compositions derived from lower in the melting column. These melts may have originated as near-fractional melts and appear to exhibit a wide variety of enriched to depleted compositions, suggesting melt transport via channelization through the melting column. Overall, the residual mantle is depleted in clinopyroxene (and $\mathrm{CaO}$ ) relative to that predicted from melting, possibly because of dissolution of clinopyroxene. Throughgoing melts appear to have also locally enriched the residual upper mantle in highly incompatible elements when it became part of the mechanical lithosphere. A reenrichment process appears to occur locally during late-stage porous flow of mantle melts when they become saturated in clinopyroxene and a later impregnation stage when magmatic dunite, clinopyroxenite, Iherzolite, websterite, wehrlite and gabbroic dikes intrude the mantle as it becomes part of the mechanical lithosphere. These intrusive rocks may involve melts that are both depleted and enriched in LREEs. Some may have undergone fractionation within a thick mechanical lithosphere $(>10$ $\mathrm{km})$.

6. The plutonic rocks show a wide range in major- and trace-element compositions that surpass most previously collected and documented oceanic plutonic suites from a single site. The wide range of trace-element compositions is attributed to modal chemistry, extent of fractionation, the percentage of trapped or infiltrating melt, and a variety of near-fractional melts derived from the melting column. Fractionation processes during solidification, migration, and mobilization of intercumulus melts appear to explain the origin of magmatic intrusions that range from oxide gabbros to quartz diorite in composition. Such a process also explains the typically large compositional gap between moderately fractionated samples similar to MARK MORB and highly fractionated oxide gabbro and dioritic samples.

7. Cryptic chemical variation is documented both in the mineral and bulk-rock chemistry within the gabbroic sections sampled at Sites 921, 922, and 923 and the peridotite holes of Site 920. The variation is on the scale of a few meters to tens of meters in gabbroic and ultramafic massif, but is also documented on a scale of less than a centimeter within pyroxenite and gabbroic dikes within the peridotite massif (Site 920). This chemistry appears correlatable when holes are closely spaced. The scale of cryptic units within gabbroic Hole $923 \mathrm{~A}$, which had the highest recovery, is best documented and appears to range from a few meters to tens of meters, with both normal and inverted fractionation trends upsection.

8. The lithologies and mineral chemistries of ultramafic and mafic plutonic rocks span the compositional range documented within ophiolites, including the ultramafic-mafic transition zone. Rather than stratigraphically atop the residual mantle, ultramafic cumulates appear dispersed throughout the residual mantle section as small and discrete intrusions that collectively form a significant component of cores from Holes 920B and $920 \mathrm{D}$.

9. The mineral chemistry of plutonic and residual rocks establish that a range of melt compositions, from ultradepleted to enriched in incompatible elements, entered the subaxial magma system in the MARK area, but their compositions have essen- tially been masked in the erupted basalts by mixing and hybridization to form MORB.

10. Finally, it appears that oceanic crustal architecture may be largely governed by magma supply rate and may lead to a variety and spectrum of crustal and upper mantle types. The MARK area crustal architecture has been affected by low melt supply and extensive mechanical extension.

\section{ACKNOWLEDGMENTS}

I thank the JOIDES Resolution shipboard and technical staff for their help in obtaining samples. I also thank the reviewers for their many constructive comments. Discussions with Mathilde Cannat, Jeff Karson, Debra Kelley, Jay Miller, Sue Agar, Pamela Kempton, Christopher Stevens, Kiyoaki Niida, and the Leg 153 Shipboard Scientific Party were very helpful before and during the preparation of this manuscript. Discussion over the last several years with Bill Bryan, Leonid Dmitriev, Henry Dick, Peter Meyer, Sergei Silantyev, Susan Smith, and Chunshou Xia on the subjects of MORB petrogenesis and abyssal peridotites and oceanic gabbros have benefited the author greatly. Tim O'Hearn and Bill Melson are thanked for their help in obtaining Mid-Atlantic Ridge glass analyses. Susan Smith is thanked her help with the ICP work. Chunshou Xia is thanked for the use of his continuous melting modeling. Rob Polkalny is thanked for providing the digital multibeam data to create sample location maps. Work on ODP samples was supported by USSAC. Part of the work Mid-Atlantic Ridge MORB and plutonic rocks reported here was supported by NSF OCE-920-4015.

\section{REFERENCES}

Anders, E., and Grevesse, N., 1989. Abundances of the elements: meteoritic and solar. Geochim. Cosmochim. Acta, 53:197-214.

Bender, J.F., Hodges, F.N., and Bence, A.E., 1978. Petrogenesis of basalts from the Project FAMOUS area: experimental study from 0 to 15 kbars. Earth Planet. Sci. Lett., 41:277-302.

Bischoff, J.L., and Seyfried, W.E., 1978. Hydrothermal chemistry of seawater from $25^{\circ} \mathrm{C}$ to $350^{\circ} \mathrm{C}$. Am. J. Sci., 278:838-860,

Bonatti, E., Lawrence, J.R., Hamlyn, P.R., and Breger, D., 1980. Aragonite from deep-sea ultramafic rocks. Geochim. Cosmochim. Acta, 44:12071214.

Bonatti, E., Lawrence, J.R., and Morandi, N., 1984. Serpentinization of ocean-floor peridotites: temperature dependence on mineralogy and boron content. Earth Planet. Sci. Lett., 70:88-94.

Bonatti, E., and Michael, P.J., 1989. Mantle peridotites from continental rifts to ocean basins to subduction zones. Earth Planet. Sci. Lett., 91:297311.

Bonatti, E., Peyve, A., Kepezhinskas, P., Kurentsova, N., Seyler, M., Skolotnev, S., and Udintsev, G., 1992. Upper mantle heterogeneity below the Mid-Atlantic Ridge, $0^{\circ}-15^{\circ}$ N. J. Geophys. Res., 97:4461-4476.

Bonatti, E., Seyler, M., and Sushevskaya, N., 1993. A cold suboceanic mantle belt at the Earth's Equator. Science, 261:315-320.

Bougault, H., Dmitriev, L., Schilling, J.G., Sobolev, A., Joron, J.L., and Needham, H.D., 1988. Mantle heterogeneity from trace elements: MAR triple junction near $14^{\circ}$ N. Earth Planet. Sci. Lett., 88:27-36.

Bougault, H., Joron, J.L., and Treuil, M., 1979. Alteration, fractional crystallization, partial melting, mantle properties from trace elements in basalts recovered in the North Atlantic. In Talwani, M., Harrison, C.G., and Hayes, D.E. (Eds.), Deep Drilling Results in the Atlantic Ocean: Ocean Crust. Am. Geophys. Union, Maurice Ewing Ser., 2:352-368.

Bryan, W.B., Humphris, S.E., Thompson, G., and Casey, J.F., 1994. Comparative volcanology of small axial eruptive centers in the MARK area. J. Geophys. Res., 99:2973-2984.

Bryan, W.B., Thompson, G., and Ludden, J.N., 1981. Compositional variation in normal MORB from $22^{\circ}-25^{\circ} \mathrm{N}$ : Mid-Atlantic Ridge and Kane Fracture Zone. J. Geophys. Res., 86:11815-11836.

Campbell, A.C., Palmer, M.R., Klinkhammer, G.P., Bowers, T.S., Edmond, J.M., Lawrence, J.R., Casey, J.F., Thompson, G., Humphris, S., Rona, 
P.A., and Karson, J.A., 1988. Chemistry of hot springs on the Mid-Atlantic Ridge. Nature, 335:514-519.

Campbell, I.H., 1978. Some problems with cumulus theory. Lithos, 11:311323.

Cannat, M., 1993. Emplacement of mantle rocks in the seafloor at mid-ocean ridges. J. Geophys. Res., 98:4163-4172.

Cannat, M., Mével, C., Maia, M., Deplus, C., Durand, C., Gente, P., Agrinier, P., Belarouchi, A., Dubuisson, G., Humler, E., and Reynolds, J., 1995. Thin crust, ultramafic exposures and rugged faulting patterns at the Mid-Atlantic Ridge $\left(22^{\circ}-24^{\circ} \mathrm{N}\right)$. Geology, 23:49-52.

Cannat, M., and Casey, J.F., 1995. An ultramafic lift at the Mid-Atlantic Ridge: successive stages of magmatism in serpentinized peridotites from the $15^{\circ} \mathrm{N}$ region. In Vissers, R.L.M., and Nicolas, A. (Eds.), Mantle and Lower Crust Exposed in Oceanic Ridges and Ophiolites: Dordrecht (Kluwer), 5-34.

Cannat, M., Karson, J.A., Miller, D.J., et al., 1995. Proc. ODP, Init. Repts., 153: College Station, TX (Ocean Drilling Program).

Cannat, M., Mével, C., Maia, M., Durand, C., Gente, P., Deplus, C., Agrinier, P., Belarouchi, A., Dubuisson, G., Humler, E., and Reynolds, J., 1993. Crustal structure and axial segmentation, Mid-Atlantic Ridge 21$24^{\circ}$ N. Eos, $74: 664$

Casey, J.F., 1980. The geology of the southern half of the North Arm Mountain Massif, Bay of Islands Ophiolite Complex, western Newfoundland, with application to ophiolite obduction and genesis of plutonic portions of the oceanic crust and upper mantle [Ph.D. dissert.]. State University of NY, Albany, NY.

Casey, J.F., 1986. Ultramafic rocks from the M.A.R. at $23^{\circ} \mathrm{N}$ : evidence for high temperature hydrations and high temperature-low to moderate stress deformation of mantle tectonites beneath the median valley. Eos, 67:1214.

Casey, J.F., Bryan, W.B., Xia, C., Smith, S., Dmitriev, L., Silantiev, S., and Melson, W.G., 1992a. Basalt compositional trends, $12^{\circ}$ to $38^{\circ} \mathrm{N}$ along the Mid-Atlantic Ridge: local paradigms. Eos, 73:584.

Casey, J.F., Cannat, M., and Bougault, H., 1992b. MAR survey opens window to offset drilling. JOI/USSAC Newsl., 5:1-3.

Casey, J.F., Dewey, J.F., Fox, P.J., Karson, J.A., and Rosencrantz, E., 1981. Heterogeneous nature of oceanic crust and upper mantle: a perspective from the Bay of Islands Ophiolite Complex. In Emiliani, C. (Ed.), The Sea (Vol. 7): The Oceanic Lithosphere: New York (Wiley), 305-338.

Casey, J.F., Elthon, D.L., Sirkoy, F.X., Karson, J.A., and Sullivan, J., 1985. Geochemical and geological evidence bearing on the origin of the Bay of Islands and Coastal Complex ophiolites of western Newfoundland. Tectonophysics, 116:1-40.

Casey, J.F., Karson, J.A., Elthon, D., Rosencrantz, E., and Titus, M., 1983. Reconstruction of the geometry of accretion during formation of the Bay of Islands Ophiolite Complex. Tectonics, 2:509-528.

Cohen, R.S., and O'Nions, R.K., 1982. The lead, neodymium and strontium isotopic structure of ocean ridge basalts. J. Petrol., 23:299-324.

Cordery, M.J., and Phipps Morgan, J., 1993. Convection and melting at MidOcean Ridges. J. Geophys. Res., 98:19477-19503.

Detrick, R.S., Fox, P.J., Schulz, N., Pockalny, R., Knog, L., Mayer, L., and Ryan, W.B.F., 1988. Geologic and tectonic setting of the mark area. In Detrick, R., Honnorez, J., et al., Proc. ODP, Init. Repts., 106/109: College Station, TX (Ocean Drilling Program), 15-22.

Dick, H.J.B., 1989. Abyssal peridotites, very slow spreading ridges and ocean ridge magmatism. In Saunders, A.D., and Norry, M.J. (Eds.), Magmatism in the Ocean Basins. Geol. Soc. Spec. Publ. London, 42:71-105.

Dick, H.J.B., and Bullen, T., 1984. Chromian spinel as a petrogenetic indicator in abyssal and alpine-type peridotites and spatially associated lavas. Contrib. Mineral. Petrol., 86:54-76.

Dick, H.J.B., and Fisher, R.L., 1984. Mineralogical studies of the residues of mantle melting: abyssal and alpine-type peridotites. In Kornprobst, J. (Ed.), Kimberlites II: The Mantle and Crust-Mantle Relationships: Amsterdam (Elsevier), 295-308.

Dick, H.J.B., Fisher, R.L., and Bryan, W.B., 1984. Mineralogic variability of the uppermost mantle along mid-ocean ridges. Earth Planet. Sci. Lett., 69:88-106.

Dick, H.J.B., and Kelemen, P.B., 1991. Fractionation of Ti from rare earth elements during formation of harzburgite from lherzolite by magma/ mantle interaction. Eos, 72:545.

Dick, H.J.B., Meyer, P.S., Bloomer, S., Kirby, S., Stakes, D., and Mawer, C., 1991. Lithostratigraphic evolution of an in-situ section of oceanic Layer
3. In Von Herzen, R.P., Robinson, P.T., et al., Proc. ODP, Sci. Results, 118: College Station, TX (Ocean Drilling Program), 439-538.

Dosso, L., Bougault, H., and Joron, J.L., 1993. Geochemical morphology of the North Atlantic Ridge, $10^{\circ}-24^{\circ} \mathrm{N}$ : trace element-isotope complementarity. Earth Planet. Sci. Lett., 120:443-462.

Dosso, L., Hanan, B.B., Bougault, H., Schilling, J.G., and Joron, J.-L., 1991. $\mathrm{Sr}-\mathrm{Nd}-\mathrm{Pb}$ geochemical morphology between $10^{\circ}$ and $17^{\circ} \mathrm{N}$ on the MidAtlantic Ridge: a new MORB isotope signature. Earth Planet. Sci. Lett., 43:46-60.

Dupré, B., and Allègre, C.J., 1980. Pb-Sr-Nd isotopic correlation and the chemistry of the North Atlantic mantle. Nature, 286:17-22.

Elthon, D., 1987. Petrology of gabbroic rocks from the Mid-Cayman Rise spreading center. J. Geophys. Res., 92:658-682.

Elthon, D., and Casey, J.F., 1985. The very depleted nature of certain primary mid-ocean ridge basalts. Geochim. Cosmochim. Acta, 49:289-298.

Elthon, D., Casey, J.F., and Komor, S., 1982. Mineral chemistry of ultramafic cumulates from the North Arm massif of the Bay of Islands ophiolite: implication for high pressure fractionation of oceanic basalts. J. Geophys. Res., 87:8717-8734.

Elthon, D., Casey, J.F., and Komor, S., 1984a. Cryptic mineral chemistry variations in a detailed traverse through the cumulate ultramafic rocks of the North Arm Mountain massif of the Bay of Islands Ophiolite, Newfoundland. In Gass, I.G., Lippard, S.J., and Shelton, A.W. (Eds.), Ophiolites and Oceanic Lithosphere: London (Blackwell), 269-290.

Elthon, D., Lawrence, J.R., Hanson, R.E., and Stern, C., 1984b. Modeling of oxygen-isotope data from Sarmiento ophiolite complex, Chile. In Gass, I.G., Lippard, S.J., and Shelton, A.W. (Eds.), Ophiolites and Oceanic Lithosphere: Oxford (Blackwell Sci. Publ.), 185-197.

Falloon, T.J., and Green, D.H., 1987. Anhydrous partial melting of MORB pyrolite and other peridotite compositions at $10 \mathrm{kbar}$ : implications for the origin of primitive MORB glasses. Contrib. Mineral. Petrol., 37:181219.

Freer, R., 1981. Diffusion in silicate minerals: a data digest and guide to the literature. Contrib. Mineral. Petrol., 76:440-454.

Frey, F.A., 1984. Rare earth element abundances in upper mantle rocks. In Henderson, P. (Ed.), Rare Earth Element Geochemistry: Amsterdam (Elsevier), 153-203.

Frey, F., 1989. Rare earth elements in the upper mantle rocks. In Lipin, B.R., and McKay, G.A., (Eds.), Geochemistry and Mineralogy of Rare Earth Elements: Washington, D.C. (Mineral. Soc. Amer.), 99-145.

Fujii, T., and Scarfe, C.M., 1985. Composition of liquids coexisting with spinel lherzolite at $10 \mathrm{kbar}$ and the genesis of MORBs. Contrib. Mineral. Petrol., 90:18-28.

Fujimaki, H., Tatsumoto, M., and Aoki, K., 1984. Partition coefficients of Hf, Zr, and REE between phenocrysts and groundmasses. Proc. Lunar Planet. Sci. Conf., 14th (Part 2). J. Geophys. Res., 89 (Suppl.):B662B672.

Gente, P., Pockalny, R., Durand, R., Deplus, C., Maia, C., Ceuleneer, M., Mével, C., Cannat, M., and Laverne, C., 1995. Characteristics and evolution of the segmentation of the Mid-Atlantic Ridge between $20^{\circ}$ and $24^{\circ} \mathrm{N}$ during the last 10 million years. Earth Planet. Sci. Lett., 129:5571.

Gente, P., Zonenshain, L.P., Kuzmin, M., Lisitsin, A.P., Bogdanov, Y.A., and Baronov, B.V., 1989. Geologie de l'axe de la dorsale médio-Atlantique entre 23 et $26^{\circ} \mathrm{N}$ : résultats préliminaires de la 15 ème campagne du N/O Akademik Mstyslav Keldysh (mars-avril 1988). C.R. Acad. Sci. Ser. 2, 308:1781-1788.

Gillis, K.M., Thompson, G., and Kelley, D., 1993. A view of the lower crustal component of hydrothermal systems at the Mid-Atlantic Ridge. $J$. Geophys. Res., 98:19597-19619.

Girardeau, J., and Francheteau, J., 1993. Plagioclase-wehrlites and peridotites on the East Pacific Rise (Hess Deep) and the Mid-Atlantic Ridge (DSDP Site 339) evidence for magma percolation in the oceanic upper mantle. Earth Planet. Sci. Lett., 115:137-149.

Girardeau, J., and Mercier, J.-C.C., 1992. Evidence for plagioclase-lherzolite intrusion in the Mid-Atlantic Ridge, DSDP Leg 37. In Parson, L.M., Murton, B.J., and Browning P. (Eds.), Ophiolites and their Modern Oceanic Analogues. Geol. Soc. Spec. Publ. London, 60:241-250.

Grove, T.L., Kinzler, R.J., and Bryan, W.B., 1992. Fractionation of midocean ridge basalt (MORB). In Morgan, J.P., Blackman, D.K., and Sinton, J.M. (Eds.), Mantle Flow and Melt Generation at Mid-Ocean Ridges. Geophys. Monogr., Am. Geophys. Union, 71:281-310. 
Hanson, G.N., 1980. Rare earth elements in petrogenetic studies of igneous systems. Ann. Rev. Earth Planet. Sci., 8:371-406.

Hart, S.R., and Dunn, T., 1993. Experimental CPX/melt partitioning of 24 trace elements. Contrib. Mineral. Petrol., 113:1-8.

Hart, S.R., and Zindler, A., 1986. In search of a bulk Earth composition. Chem. Geol., 57:247-267.

Helmley, J.J., Montoya, J.W., Christ, C.L., and Hostetler, P.B., 1977. Mineral equilibria in the $\mathrm{MgO}-\mathrm{SiO}_{2}-\mathrm{H}_{2} \mathrm{O}$ System I: talc-chrysotile-forsteritebrucite stability relations. Am. J. Sci., 277:322-351.

Hemond, C., Arndt, N.T., Lichtenstein, U., Hoffman, A.W., Oskarsson, N., and Steintthorsson, S., 1993. The heterogeneous Iceland plume: $\mathrm{Nd}: \mathrm{Sr}: \mathrm{O}$ isotopes and trace element constraints. J. Geophys. Res., 98:1583315850.

Hess, P.C., 1992. Phase equilibria constraints on the origin of ocean floor basalts. In Phipps Morgan, J., Blackman, D.K., and Sinton, J.M. (Eds.), Mantle Flow and Melt Generation at Mid-Ocean Ridges. Am. Geophys. Union, Geophys. Monogr., 71:67-102.

Humphris, S., 1984. The mobility of rare earth elements in the crust. In Henderson, P. (Ed.), Rare Earth Element Geochemistry: Amsterdam (Elsevier), 317-342.

Irvine, T.N., 1979. Rocks whose composition is determined by crystal accumulation and sorting. In Yoder, H.S. (Ed.), The Evolution of the Igneous Rocks: Princeton, NJ (Princeton Univ. Press), 245-306.

Irving, A.J., 1978. A review of experimental studies of crystal/liquid trace element partitioning. Geochim. Cosmochim. Acta, 42:743-770.

Ito, E., White, W.M., and Göpel, C., 1987. The O, Sr, Nd and $\mathrm{Pb}$ isotope geochemistry of MORB. Chem. Geol., 62:157-176.

Jagoutz, E., Palme, H., Baddenhausen, H., Blum, K., Cendales, M., Dreibus, G., Spettel, B., Lorentz, V., and Wanke, H., 1979. The abundances of major, minor, and trace elements in the earth's mantle as derived from primitive ultramafic nodules. Proc. Lunar Planet. Sci. Conf., 10:20312050.

Janecky, D.R., 1982. Serpentinization of peridotite within the ocean crust: experimental and theoretical investigation of seawater-peridotite interaction at $200^{\circ} \mathrm{C}$ and $300^{\circ} \mathrm{C}, 500$ bars. Am. J. Sci., 283:831-860.

Janecky, D.R., and Seyfried, W.E., 1983. The solubility of magnesiumhydroxide-sulfate-hydrate in seawater at elevated temperatures and pressures. Am. J. Sci., 283:831-860.

Janecky, D.R., and Seyfried, W.E., Jr., 1986. Hydrothermal serpentinization of peridotite within the oceanic crust: experimental investigations of mineralogy and major element chemistry. Geochim. Cosmochim. Acta, 50:1357-1378

Jaques, A.L., and Green, D.H., 1980. Anhydrous melting of peridotite at 0$15 \mathrm{~kb}$ pressure and the genesis of the tholeitic basalts. Contrib. Mineral. Petrol., 73:287-310.

Johnson, K.T.M., and Dick, H.J.B., 1992. Open system melting and temporal and spatial variation of peridotite and basalt at the Atlantis II fracture zone. J. Geophys. Res., 97:9219-9241.

Johnson, K.T.M., Dick, H.J.B., and Shimizu, N., 1990. Melting in the oceanic upper mantle: an ion microprobe study of diopsides in abyssal peridotites. J. Geophys. Res., 95:2661-2678.

Juteau, T., Berger, E., and Cannat, M., 1990. Serpentinized, residual mantle peridotites from the M.A.R. median valley, ODP Hole $670 \mathrm{~A}\left(21^{\circ} 10^{\prime} \mathrm{N}\right.$, $45^{\circ} 02^{\prime} \mathrm{W}$, Leg 109): primary mineralogy and geothermometry. In Detrick, R., Honnorez, J., Bryan, W.B., Juteau, T., et al., Proc. ODP, Sci. Results, 106/109: College Station, TX (Ocean Drilling Program), 27-45.

Karson, J.A., 1990. Seafloor spreading on the Mid-Atlantic Ridge: implications for the structure of ophiolites and oceanic lithosphere produced in slow-spreading environments. In Malpas, J., Moores, E.M., Panayiotou, A., and Xenophontos, C. (Eds.), Ophiolites: Oceanic Crustal Analogues: Proc. Symp. "Troodos 1987": Nicosia, Cyprus (Minist. Agric. Nat. Resour.), 547-555.

Karson, J.A., and Dick, H.J.B., 1983. Tectonics of ridge-transform intersections at the Kane Fracture Zone. Mar. Geophys. Res., 6:51-98.

Karson, J.A., Thompson, G., Humphris, S.E., Edmond, J.M., Bryan, W.B., Brown, J.R., Winters, A.T., Pockalny, R.A., Casey, J.F., Campbell, A.C., Klinkhammer, G., Palmer, M.R., Kinzler, R.J., and Sulanowska, M.M., 1987. Along-axis variations in seafloor spreading in the MARK area. Nature, 328:681-685.

Kelemen, P.B., Dick, H.J.B., and Quick, J.E., 1992. Formation of harzburgite by pervasive melt/rock reaction in the upper mantle. Nature, 358:635641 .

Kelemen, P.B., Shimizu, N., and Dunn, T., 1993. Relative depletion of niobium in some arc magmas and the continental crust: partitioning of $\mathrm{K}$,
$\mathrm{Nb}, \mathrm{La}$, and Ce during melt/rock reaction in the upper mantle. Earth Planet. Sci. Lett., 120:111-133.

Kinzler, R.J., and Grove, T.L., 1992. Primary magmas of mid-ocean ridge basalts, 1. Experiments and methods. J. Geophys. Res., 97:6885-6906.

Klein, E.M., and Langmuir, C.H., 1987. Global correlations of ocean ridge basalt chemistry with axial depth and crustal thickness. J. Geophys. Res., 92:8089-8115.

Komor, S., Elthon, D., and Casey, J.F., 1985a. Cryptic variations in layered cumulate ultramafic rocks of the North Arm massif, Bay of Islands ophiolite, Newfoundland: characteristics of magmatic processes in the lower portions of mid-ocean ridge magma chambers. J. Geophys. Res., 90:7705-7736.

Komor, S.C., Elthon, D.E., and Casey, J.F., 1985b. Serpentinization of cumulate ultramafic rocks from the North Arm Mountain massif of the Bay of Islands ophiolite. Geochim. Cosmochim. Acta, 49:2331-2338.

Komor, S.C., Elthon, D., and Casey, J.F., 1987. Petrology of a leucogabbroic interval within basal layered gabbros at North Arm Mountain, Bay of Islands Ophiolite. Contrib. Mineral. Petrol., 95:278-300.

Komor, S.C., Grove, T.L., and Hébert, R., 1990. Abyssal peridotites from ODP Hole $670 \mathrm{~A}\left(21^{\circ} 10^{\circ} \mathrm{N}, 45^{\circ} 02 \mathrm{~W}\right)$ : residues of mantle melting exposed by non-constructive axial divergence. In Detrick, R., Honnorez, J., Bryan, W.B., Juteau, T., et al., Proc. ODP, Sci. Results, 106/109: College Station, TX (Ocean Drilling Program), 85-101.

Lane, R., 1988. Mineralogy and stable isotope geochemistry of serpentinized ultramafic rocks from the Bay of Islands Ophiolite Complex: implications for the oceanic Moho [M.S. thesis]. Univ. of Houston, Houston, TX.

Langmuir, C.H., Klein, E., and Plank, T., 1992. Petrological systematics of mid-ocean ridge basalts: constraints on melt generation beneath ocean ridges. In Morgan, J., Blackman, D., Sinton, J. (Eds.), Mantle Flow and Melt Generation at Mid-Ocean Ridges. Geophys. Monogr., Am. Geophys. Union, 71:183-277.

Lindsley, D.H., and Andersen, D.J., 1983. A two-pyroxene thermometer. $J$. Geophys. Res., 88 (Suppl.):A887-A906.

Ludden, J.N., and Thompson, G., 1979. An evaluation of the behavior of the rare earth elements during the weathering of sea floor basalt. Earth Planet. Sci. Lett., 43:85-92.

Lytwyn, J., and Casey, J.F., 1993. The geochemistry and petrogenesis of volcanics and sheeted dikes in the Hatay (Kizildag) ophiolite, southern Turkey: possible formation with the Troodos ophiolite, Cyprus along forearc spreading centers. Tectonophysics, 223:237-272

Lytwyn, J., and Casey, J.F., 1995. The geochemistry and petrogenesis of post-kinematic mafic dike swarms and metamorphic sole amphibolites, Pozanti-Karsanti ophiolite, Turkey: evidence for ridge subduction, Geol. Soc. Am. Bull., 107:830-850.

Machado, N., Ludden, J.N., Brooks, C., and Thompson, G., 1982. Fine-scale isotopic heterogeneity in the sub-Atlantic mantle. Nature, 295:226-228.

McDonough, W.F., and Frey, F.A., 1989. Rare earth elements in upper mantle rocks. In Lipin, B.R., and McKay, G.A. (Eds.), Geochemistry and Mineralogy of Rare Earth Elements. Rev. Mineral., 21:99-145.

McKay, G.A., 1986. Crystal/liquid partitioning of REE in basaltic systems: extreme fractionation of REE in olivine. Geochim. Cosmochim. Acta, $50: 69-79$.

McKenzie, D., 1984. The generation and compaction of partially molten rock. J. Petrol., 25:713-765.

McKenzie, D., 1985. The extraction of magma from the crust and mantle. Earth Planet. Sci. Lett., 74:81-91.

McKenzie, D., and Bickle, M.J., 1988. The volume and composition of melt generated by extension of the lithosphere. J. Petrol., 29:625-679.

Melson, W.G., and O'Hearn, T., 1979. Basaltic glass erupted along the MidAtlantic Ridge between $0^{\circ}$ and $37^{\circ} \mathrm{N}$ : relationships between composition and latitude. In Talwani, M., Harrison, C.G., and Hayes, D.E. (Eds.), Deep Drilling Results in the Atlantic Ocean: Ocean Crust. Am. Geophys. Union, Maurice Ewing Ser., 2:249-261.

, 1986. "Zero-age" variations in the compositions of abyssal volcanic rocks along the axial zone of the Mid-Atlantic ridge. In. Vogt, P.R., and Tucholke, B.E. (Eds.), The Western North Atlantic Region. Geol. Soc. Am., Geol. of North Am. Ser., M:17-136.

Melson, W.G., Thompson, W.G., and van Andel, T.H., 1968. Volcanism and metamorphism in the Mid-Atlantic Ridge, $22^{\circ} \mathrm{N}$. J. Geophys. Res., 73:5925-5941.

Menzies, M., Blanchard, D., Brannon, J., and Korotev, R., 1977. Rare earth geochemistry of fused ophiolitic and alpine lherzolites II. Beni Bouchera, Ronda and Lanzo. Contrib. Mineral. Petrol., 64:53-74. 
Menzies, M.A., Long, A., Ingram, G., Tatnell, M., and Janecky, D., 1993. MORB peridotite-sea water interaction: experimental constraints on the behaviour of trace elements, ${ }^{87} \mathrm{Sr} /{ }^{86} \mathrm{Sr}$ and ${ }^{143} \mathrm{Nd} /{ }^{144} \mathrm{Nd}$ ratios. In Prichard, H.M., Alabaster, T., Harris, N.B.W., and Neary, C.R. (Eds.), Magmatic Processes and Plate Tectonics. Geol. Soc. Spec. Publ. London, 76:309322.

Mertz, D., Devey, C., Todt, W., Stoffers, P., and Hofmann, A., 1991. Sr-Nd$\mathrm{Pb}$ isotope evidence against plume-asthenosphere mixing north of Iceland. Earth Planet. Sci. Lett., 107:243-255.

Mével, C., Cannat, M., Gente, P., Marion, E., Auzende, J.-M., and Karson, J.A., 1991. Emplacement of deep crustal and mantle rocks on the west median valley wall of the MARK area (MAR $23^{\circ} \mathrm{N}$ ). Tectonophysics, 190:31-53.

Meyer, P.S., Dick, H.J.B., and Thompson, G., 1989. Cumulate gabbros from the Southwest Indian Ridge, $54^{\circ} \mathrm{S}-7^{\circ} 16^{\prime} \mathrm{E}$ : implications for magmatic processes at a slow spreading ridge. Contrib. Mineral. Petrol., 103:4463.

Michael, P.J., and Bonatti, E., 1985. Peridotite composition from the North Atlantic: regional and tectonic variations and implications for partial melting. Earth Planet. Sci. Lett., 73:91-104.

Miyashiro, A., Shido, F., and Ewing, M., 1969. Composition and origin of serpentinites from the Mid-Atlantic Ridge, $24^{\circ}$ and $30^{\circ} \mathrm{N}$ latitude. Contrib. Mineral. Petrol., 23:117-127.

Mottl, M., 1976. Chemical exchange between seawater and basalt during hydrothermal alteration of the oceanic crust [Ph.D. dissert.], Harvard Univ., Cambridge, MA.

Natland, J.H., Meyer, P.S., Dick, H.J.B., and Bloomer, S.H., 1991. Magmatic oxides and sulfides in gabbroic rocks from Hole 735B and the later development of the liquid line of descent. In Von Herzen, R.P., Robinson, P.T., et al., Proc. ODP, Sci. Results, 118: College Station, TX (Ocean Drilling Program), 75-111.

Nesbitt, H.W., and Wilson, R.E., 1992. Recent chemical weathering of basalts. Am. J. Sci., 292:740-777.

Nicolas, A., 1986. A melt extraction model based on structural studies in mantle peridotites. J. Petrol., 27:999-1022.

Niu, Y., and Batiza, R., 1991. An empirical method for calculating melt compositions produced beneath mid-ocean ridges: application for axis and off-axis (seamounts) melting. J. Geophys. Res., 96:21753-21777.

Ozawa, K., Meyer, P.S., and Bloomer, S.H., 1991. Mineralogy and textures of iron-titanium oxide gabbros and associated olivine gabbros from Hole 735B. In Von Herzen, R.P., Robinson, P.T., et al., Proc. ODP, Sci. Results, 118: College Station, TX (Ocean Drilling Program), 41-73.

Pallister, J.S., and Hopson, C.A., 1981. Samail ophiolite plutonic suite: field relations, phase variation, cryptic variation and layering, and a model of a spreading ridge magma chamber. J. Geophys. Res., 86:2593-2644.

Pearce, J.A., and Parkinson, I.J., 1993. Trace element models for mantle melting: application to volcanic arc petrogenesis. In Pritchard, H.M., Alabaster, T., Harris, N.B.W., and Neary, C.R. (Eds.), Magmatic Processes and Plate Tectonics. Geol. Soc. Spec. Publ. London, 76:373-403.

Philpotts, J.A., and Schnetzler, C.C., 1970. Phenocryst-matrix partition coefficients for $\mathrm{K}, \mathrm{Rb}, \mathrm{Sr}$, and $\mathrm{Ba}$ with applications to anorthosite and basalt genesis. Geochim. Cosmochim. Acta, 34:307-322.

Phinney, W.C., and Morrison, D.A., 1990. Partition coefficients for calcic plagioclase: implications for Archean anorthosites. Geochim. Cosmochim. Acta, 54:1639-1654.

Plank, T., and Langmuir, C.H., 1992. Effects of the melting regime on the composition of the oceanic crust. J. Geophys. Res., 97:19749-19770.

Plank, T., Spiegelman, M., Langmuir, C.H., and Forsyth, D.W., 1995. The meaning of "mean F": clarifying the mean extent of melting at ocean ridges. J. Geophys. Res., 100:15045-15052.

Roeder, P.L., and Emslie, R.F., 1970. Olivine-liquid equilibrium. Contrib. Mineral. Petrol., 29:275-289.

Ross, K., and Elthon, D., 1993. Cumulates from strongly depleted midocean-ridge basalt. Nature, 365:826-829.

Schilling, J.-G., 1973. Iceland mantle plume, geochemical evidence along the Reykjanes Ridge. Nature, 242:565-571.

Schilling, J.-G., Hanan, B.B., McCully, B., Kingsley, R.H., and Fontignie, D., 1994. Influence of the Sierra Leone mantle plume on the equatorial Mid-Atlantic Ridge: A Nd-Sr-Pb isotopic study. J. Geophys. Res., 99:12005-12028.

Schilling, J.-G., Zajac, M., Evans, R., Johnston, T., White, W., Devine, J.D., and Kingsley, R., 1983. Petrologic and geochemical variations along the Mid-Atlantic ridge from $29^{\circ} \mathrm{N}$ to $73^{\circ} \mathrm{N}$. Am. J. Sci., 283:510-586.
Schilling, J.-G., 1986. Geochemical and isotopic variation along the MidAtlantic Ridge axis from $79^{\circ} \mathrm{N}$ to $0^{\circ} \mathrm{N}$. In . Vogt, P.R., and Tucholke, B.E. (Eds.), The Western North Atlantic Region. Geol. Soc. Am., Geol. of North Am. Ser., M:137-153.

Schilling, J.-G., Ruppel, C., Davis, A.N., McCully, B., Tighe, S.A., Kingsley, R.H., and Lin, J., 1995. Thermal structure of the mantle beneath the equatorial Mid-Atlantic Ridge: inferences from the spatial variation of dredged basalt glass compositions. J. Geophys. Res., 100:10057-10076.

Seyfried, W.E., Jr., and Dibble, W.E., Jr., 1980. Seawater-peridotite interaction at $300^{\circ} \mathrm{C}$ and 500 bars: implications for the origin of oceanic serpentinites. Geochim. Cosmochim. Acta, 44:309-321.

Shipboard Scientific Party, 1995. Site 920. In Cannat, M., Karson, J.A., Miller, D.J., et al., Proc. ODP, Init. Repts., 153: College Station, TX (Ocean Drilling Program), 45-119.

Shirey, S.B., Bender, J.F., and Langmuir, C.H., 1987. Three-component isotopic heterogeneity near the Oceanographer transform, Mid-Atlantic Ridge. Nature, 325:217-223.

Siefert, K.E., Cole, M.R.W., and Brunnotte, D.A., 1987. REE mobility due to alteration of Indian Ocean basalt. Canad. J. Earth Sci., 22:1884-1887.

Sigurdsson, H., 1981. First-order major element variation in basalt glasses from the Mid-Atlantic Ridge: $29^{\circ} \mathrm{N}$ to $73^{\circ} \mathrm{N}$. J. Geophys. Res., 86:94839502 .

Silant'ev, S.A., 1995. Metamorphism of contemporary oceanic basins. Petrology, 3:20-30.

Smith, S.E., 1994. Geochemistry and petrology of basaltic and plutonic rocks from the Hayes Transform Region, Mid-Atlantic Ridge [Ph.D. dissert.]. Univ. of Houston, Houston, TX.

Smith, S.E., Casey, J.F., and Bryan, W.B., 1995. Evidence for wide range in parental melt compositions in the Hayes Transform Region $\left(33^{\circ} 40^{\prime} \mathrm{N}\right)$, Mid-Atlantic Ridge. Eos, 76:698.

Smith, S.E., Casey, J.F., Bryan, W.B., Silantiev, S., and Dmitriev, L., 1991. Geochemistry of basalts recovered from the Hayes Fracture Zone $\left(33^{\circ} 40^{\prime} \mathrm{N}\right)$, Mid-Atlantic Ridge. Eos, 72:517.

Snow, J.E., and Dick, H.J.B., 1995. Pervasive magnesium loss by marine weathering of peridotite. Geochim. Cosmochim. Acta, 59:4219-4235.

Sobolev, A.V., Casey, J.F., and Shimizu, N., 1992. Contamination and mixing of MORB primary melts: evidence from the Siquieros picrites. Eos, 73:336.

Sobolev, A.V., and Shimizu, N., 1992. Ultra-depleted melts and permeability of the oceanic mantle. Dokl. Acad. Sci. Russia, 236:354-360. (in Russian)

Sobolev, A.V., and Shimizu, N., 1993. Ultra-depleted primary melt included in an olivine from the Mid-Atlantic Ridge. Nature, 363:151-154.

Song, Y., and Frey, F.A., 1989. Geochemistry of peridotite xenoliths in basalts from Hannuoba, Eastern China: implications for subcontinental mantle heterogeneity. Geochim. Cosmochim. Acta, 53:97-113.

Stakes, D.S., Shervais, J.W., and Hopson, C.A., 1984. The volcanic-tectonic cycle of the FAMOUS and AMAR valleys, Mid-Atlantic Ridge $\left(36^{\circ} 47 \mathrm{~N}\right)$ : evidence from basalt glass and phenocryst compositional variations for a steady state magma chamber beneath the valley mid-sections, AMAR 3. J. Geophys. Res., 89:6995-7028.

Stosch, H.-G., 1982. Rare earth element partitioning between minerals from anhydrous spinel peridotite xenoliths. Geochim. Cosmochim. Acta, 46:793-811.

Sun, S.-S., 1980. Lead isotopic study of young volcanic rocks from midocean ridges, ocean islands and island arcs. Philos. Trans. R. Soc. London A, 297:409-445.

Sun, S.-S., and McDonough, W.F., 1989. Chemical and isotopic systematics of oceanic basalts: implications for mantle composition and processes. In Saunders, A.D., and Norry, M.J. (Eds.), Magmatism in the Ocean Basins. Geol. Soc. Spec. Publ. London, 42:313-345.

Tiezzi, L.J., and Scott, R.B., 1980. Crystal fractionation in a cumulate gabbro, Mid-Atlantic Ridge, $26^{\circ}$ N. J. Geophys. Res., 85:5438-5454.

Toomey, D.R., Solomon, S.C., and Purdy, G.M., 1988. Microearthquakes beneath the median valley of the Mid-Atlantic Ridge near $23^{\circ} \mathrm{N}$ : tomography and tectonics. J. Geophys. Res., 93:9093-9112.

Toomey, D.R., Solomon, S.C., Purdy, G.M., and Murray, M.H., 1985. Microearthquakes beneath the median valley of the Mid-Atlantic ridge near $23^{\circ} \mathrm{N}$ : hypocenters and focal mechanisms. J. Geophys. Res., 90:5443-5458.

Tormey, D.R., Grove, T.L., and Bryan, W.B., 1987. Experimental petrology of normal MORB near the Kane Fracture Zone: $22^{\circ}-25^{\circ} \mathrm{N}$, Mid-Atlantic Ridge. Contrib. Mineral. Petrol., 96:121-139. 
Wager, L.R., and Brown, G.M., 1968. Layered Igneous Rocks: London (Oliver and Boyd).

Wager, L.R., Brown, G.M., and Wadsworth, W.J., 1960. Types of igneous cumulates. J. Petrol., 1:73-85.

Weill, D.F., and McKay, G.A., 1975. The partitioning of Mg, Fe, Sr, Ce, Sm, $\mathrm{Eu}$, and $\mathrm{Yb}$ in lunar igneous systems and a possible origin of KREEP by equilibrium partial melting. Proc. 6th Lunar Sci. Conf., 1143-1158.

White, W.M., and Schilling, J-G., 1978. The nature and origin of geochemical variation in Mid-Atlantic Ridge basalts from the central north Atlantic. Geochim. Cosmochim. Acta, 42:1501-1516.
Xia, C., 1995. Geochemical variations, source characterization, mantle melting and magmatic process at the $15^{\circ} 20^{\prime} \mathrm{N}$ Fracture Zone, Mid-Atlantic Ridge [Ph.D. dissert.]. Univ. of Houston, Houston, TX.

Date of initial receipt: 4 August 1995

Date of acceptance: 14 May 1996

Ms 153SR-012 